
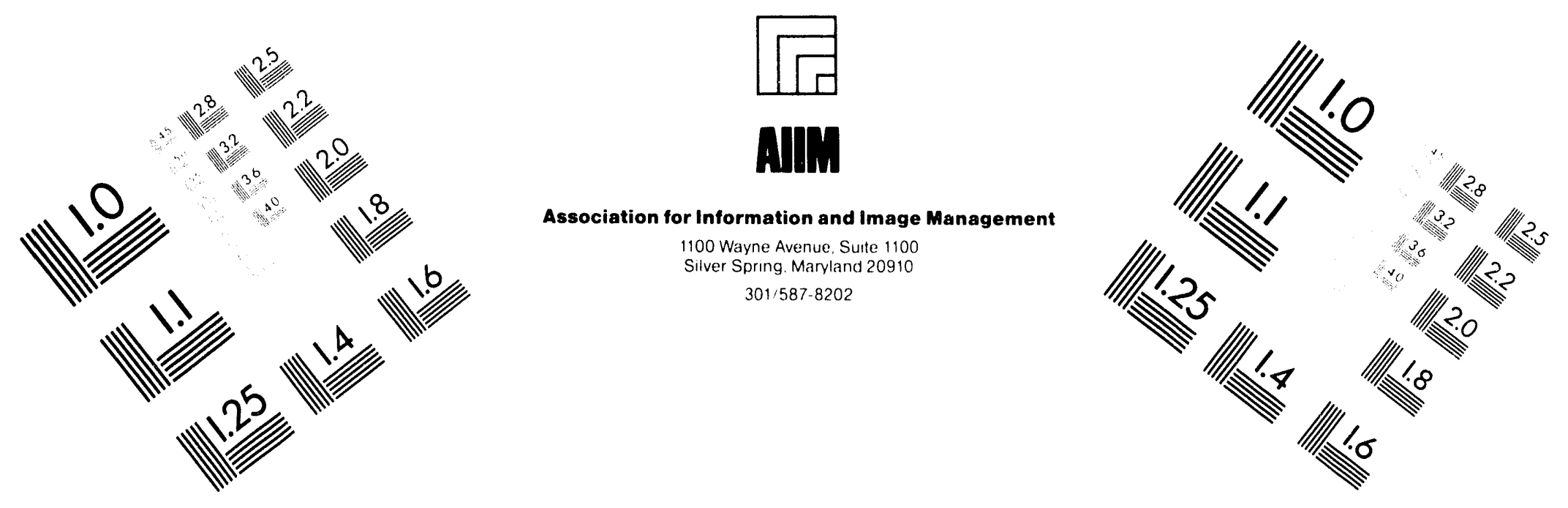

\title{
Centimeter
}

$\left.\right|_{1}$ Inches
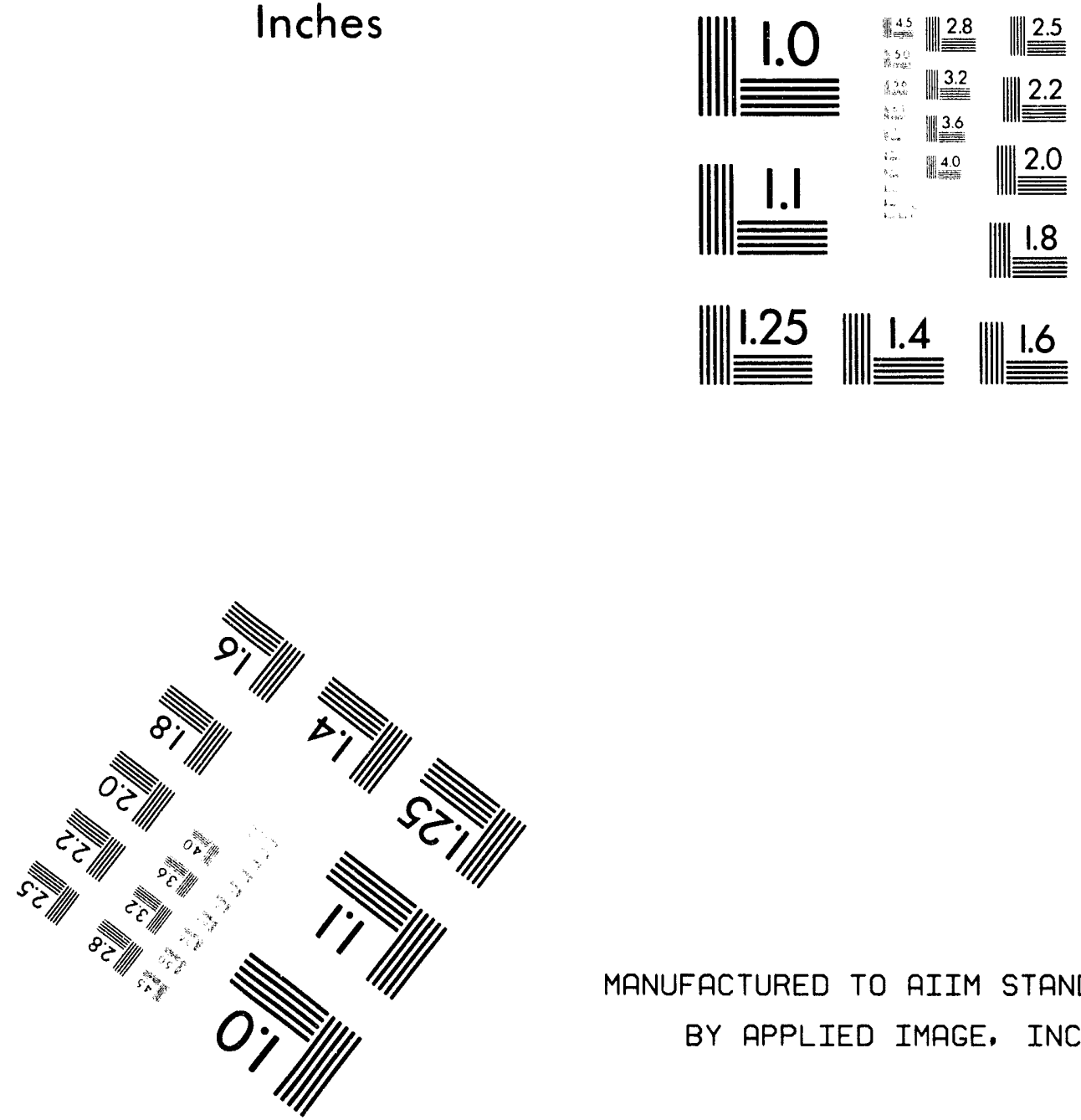

MANUFACTURED TO AIIM STANDARDS

BY APPLIED IMAGE. INC.

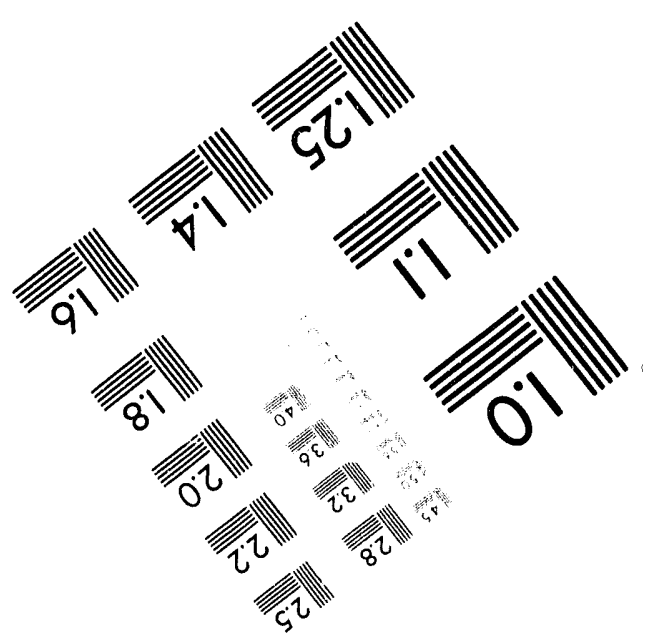



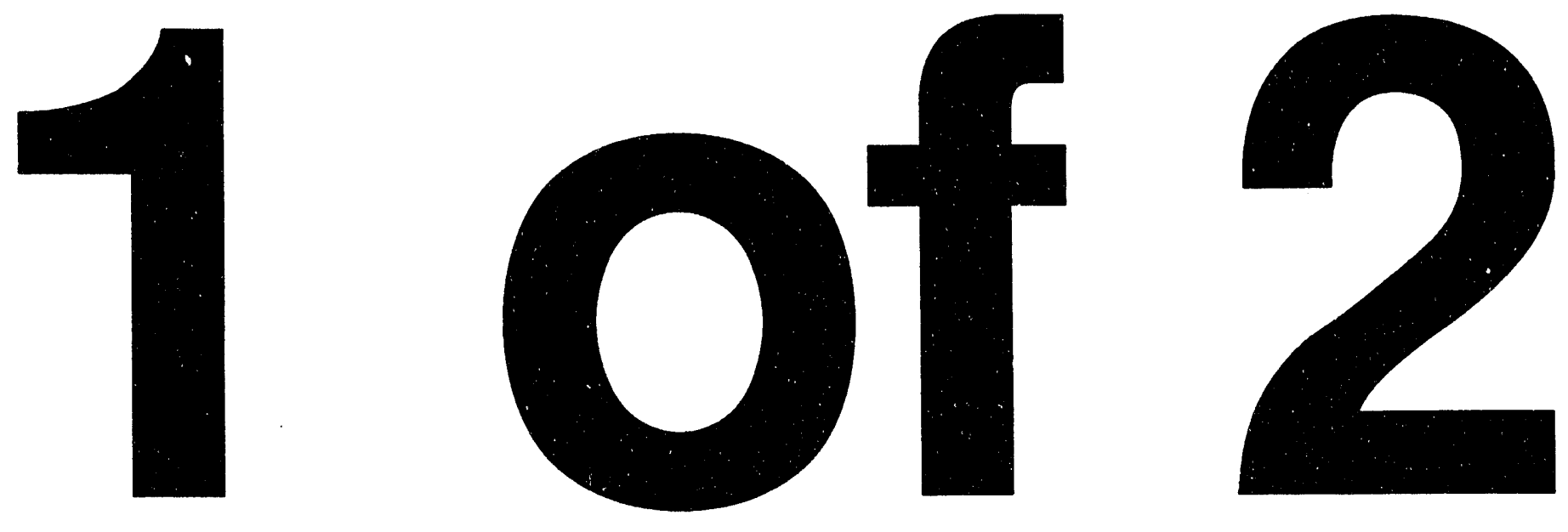
NUREG/CR-6094

BNL-NUREG-52387

\section{Calculations in Support of a Potential Definition of Large Release}

Manuscript Completed: March 1994

Date Published: May 1994

Prepared by

A. L. Hanson, R. E. Davis, V. Mubayi

Brookhaven National Laboratory

Upton, NY 11973-5000

\section{Prepared for}

Division of Safety Issues Research

Office of Nuclear Regulatory Research

U.S. Nuclear Regulatory Commission

Washington, DC 20555-0001

NRC FIN B6331 


\begin{abstract}
The Nuclear Regulatory Commission has stated a hierarchy of safety goals with the qualitative safety goals as Level I of the hierarchy, backed up by the quantitative health objectives as Level II and the large release guideline as Level III. The large release guideline has been stated in qualitative terms as a magnitude of release of the core inventory whose frequency should not exceed $10^{-6}$ per reactor year. However, the Commission did not provide a quantitative specification of a large release. This report describes various specifications of a large release and focises, in particular, on an examination of releases which have a potential to lead to one prompt fatality in the meas.

The basic information required to set up the calculations was derived from the simplified source terms which were obtained from approximations of the NUREG-1150 source terms. Since the calculation of consequences is affected by a large number of assumptions, a generic site with a (conservatively determined) population density and meteorology was specified. At this site, various emergency responses (including no response) were assumed based on information derived from earlier studies. For each of the emergency response assumptions, a set of calculations were performed with the simplified source terms; these included adjustments to the source terms, such as the timing of the release, the core inventory, and the release fractions of different radionuclides, to arrive at a result of one mean prompt fatality in each case. Each of the source terms, so defined, has the potential to be a candidate for a large release. The calculations show that there are many possible candidate source terms for a large release depending on the characteristics which are felt to be important.
\end{abstract}




\section{Contents}

Abstract $\quad \ldots \ldots \ldots \ldots \ldots \ldots \ldots \ldots \ldots \ldots \ldots \ldots \ldots \ldots \ldots \ldots \ldots \ldots \ldots \ldots \ldots \ldots \ldots$ iii

List of Figures $\ldots \ldots \ldots \ldots \ldots \ldots \ldots \ldots \ldots \ldots \ldots \ldots \ldots \ldots \ldots \ldots \ldots$ vi

List of Tables $\ldots \ldots \ldots \ldots \ldots \ldots \ldots \ldots \ldots \ldots \ldots \ldots \ldots \ldots \ldots \ldots \ldots$ vii

Executive Summary $\ldots \ldots \ldots \ldots \ldots \ldots \ldots \ldots \ldots \ldots \ldots \ldots \ldots \ldots \ldots \ldots \ldots$

Acknowledgements $\ldots \ldots \ldots \ldots \ldots \ldots \ldots \ldots \ldots \ldots \ldots \ldots \ldots \ldots \ldots \ldots \ldots \ldots \ldots \ldots i i$

1.0 Introduction $\ldots \ldots \ldots \ldots \ldots \ldots \ldots \ldots \ldots \ldots \ldots \ldots \ldots \ldots \ldots \ldots \ldots \ldots \ldots \ldots$

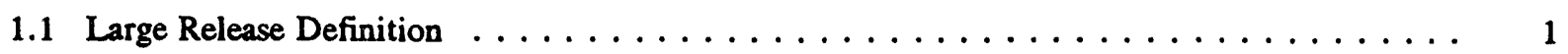

2.0 Factors Affecting Early Fatality Calculation $\ldots \ldots \ldots \ldots \ldots \ldots \ldots \ldots \ldots \ldots \ldots$

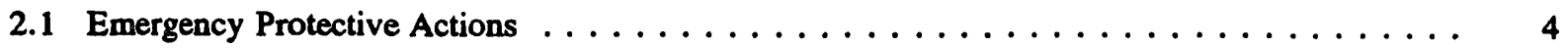

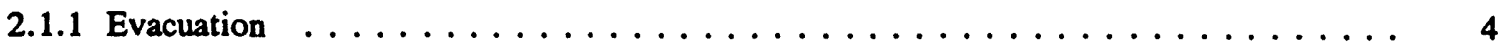

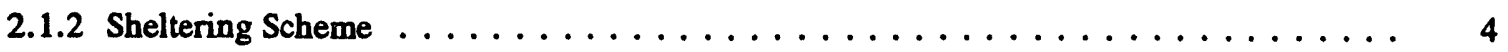

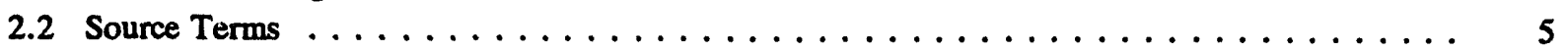

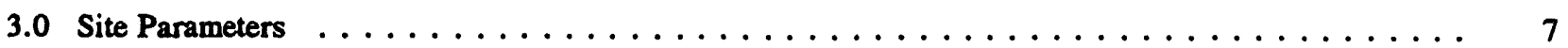

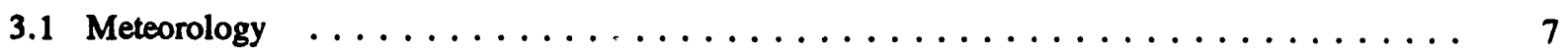

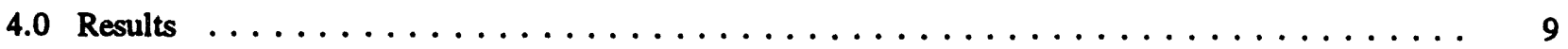

4.1 Source Terms for the No Evacuation Case $\ldots \ldots \ldots \ldots \ldots \ldots \ldots \ldots \ldots$

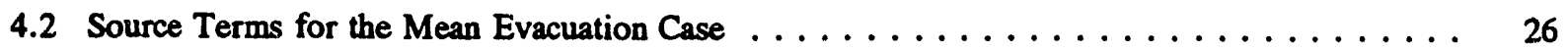

4.3 Source Terms for the Conservative Evacuation Case $\ldots \ldots \ldots \ldots \ldots \ldots$

4.4 Scaling Source Terms for the Sheltering Case $\ldots \ldots \ldots \ldots \ldots \ldots \ldots \ldots \ldots \ldots$

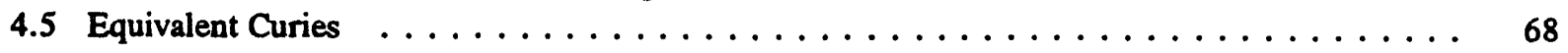

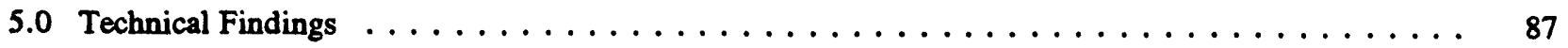

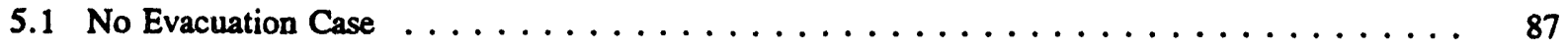

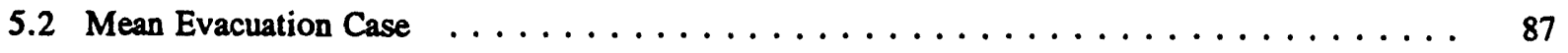

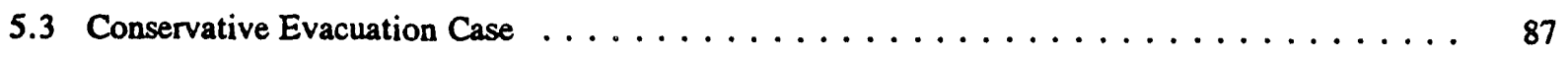

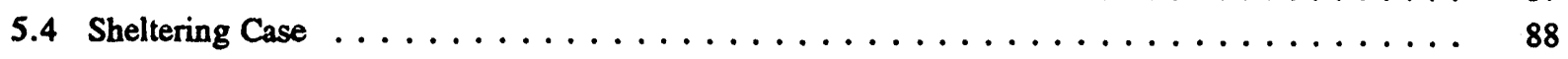

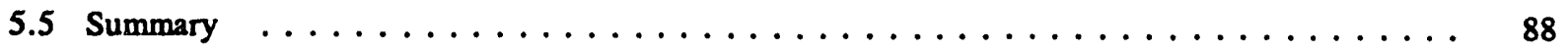

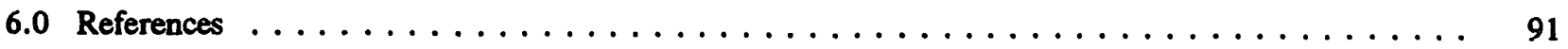

Appendix A - Simplified Source Terms $\ldots \ldots \ldots \ldots \ldots \ldots \ldots \ldots \ldots \ldots \ldots \ldots \ldots$

Appendix B - Auxiliary Dose Information $\ldots \ldots \ldots \ldots \ldots \ldots \ldots \ldots \ldots \ldots \ldots$ 


\section{List of Figures}

Page

2.0-1 Early Fatality Parameter Space $\ldots \ldots \ldots \ldots \ldots \ldots \ldots \ldots \ldots \ldots \ldots \ldots \ldots$

4.5-1 Estimated Number of Fatalities Between 0.4-1.4 Miles $\ldots \ldots \ldots \ldots \ldots \ldots \ldots \ldots$

4.5-2 Whole Body Dose at Site Boundary as Function of I-131 Release $\ldots \ldots \ldots \ldots \ldots \ldots$

4.5-3 Population Weighted Risk for Early Fatalities at Site Boundary

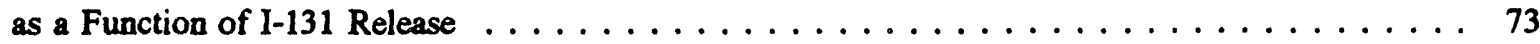

4.5-4 Estimated Number of Early Fatalities Between 0.4-1.4 Miles $\ldots \ldots \ldots \ldots \ldots \ldots \ldots$ 


\section{List of Tables}

2.1.1 EP Parameters $\ldots \ldots \ldots \ldots \ldots \ldots \ldots \ldots \ldots \ldots \ldots \ldots \ldots \ldots \ldots \ldots \ldots$

2.1.2 Shielding and Sheltering Dose Reduction Parameters $\ldots \ldots \ldots \ldots \ldots \ldots \ldots \ldots$

4.0.1 Default (3578 MWth) and Adjusted (3800 MWth) Shutdown Core Inventory for a BWR Reactor $\ldots \ldots \ldots \ldots \ldots \ldots \ldots \ldots \ldots \ldots \ldots \ldots \ldots$

4.0.2 Default (3412 (MWth) and Adjusted (3800 MWth) Shutdown Core Inventory

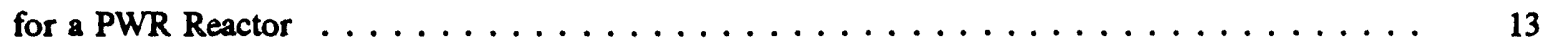

4.1.1 Mean Early Fatalities Estimated by MACCS Within One Mile Beyond

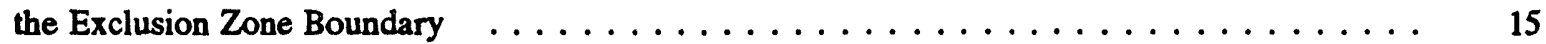

4.1.2 Changes to the Candidate Source Terms to Potentially Lead to One Early Fatality in the Mean as Estimated by MACCS $\ldots \ldots \ldots \ldots \ldots \ldots \ldots \ldots$

4.1.3 Number of Early Fatalities (after Modification) as Estimated by MACCS . . . . . . . . . . 17

4.1.4 Inventory Released Compared to the Shutdown Inventory for the Three Modified BWR Source Terms (No Evacuation Case) $\ldots \ldots \ldots \ldots \ldots \ldots$

4.1.5 Fraction of the Inventory Released for the Three Modified BWR

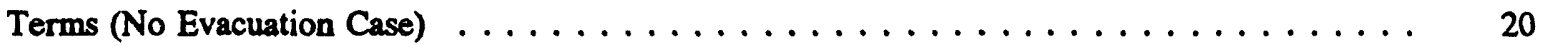

4.1.6 Inventory Released Compared to the Shutdown Inventory for the Three Modified BWR Source Terms Grouped by Isotope Grouping (No Evacuation Case) . . . . . . . . . . 22

4.1.7 Fraction of Shutdown Inventory Released for the Three Modified BWR Source Terms Grouped by Isotope Grouping (No Evacuation Case) $\ldots \ldots \ldots \ldots \ldots$

4.1.8 Inventory Released Compared to the Shutdown Core Inventory for the Modified PWR Source Term (No Evacuation Case) $\ldots \ldots \ldots \ldots \ldots \ldots \ldots \ldots \ldots \ldots \ldots \ldots \ldots$

4.1.9 Inventory Released Inventory and Fractional Release for the Modified PWR Source Term Grouped by Isotope Grouping (No Evacuation Case) $\ldots \ldots \ldots \ldots \ldots \ldots$

4.2.1 Estimated Number of Mean Early Fatalities 1 Mile Beyond Exclusion Boundary . . . . . . . 28

4.2.2 Changes to the Three Selected Source Terms to Effect One Early Fatality

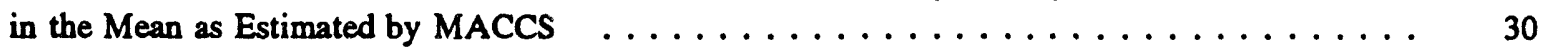

4.2.3 Mean Early Fatalities for the Three Source Terms, Initial Inventories Adjusted to Result in 1 Early Fatality for the Mean Evacuation $\ldots \ldots \ldots \ldots \ldots$

4.2.4 Inventory Released and Shutdown Inventory by Individual Isotope for the Modified BWR Source Terms . . . . . . . . . . . . . . . . . . 31

4.2.5 Inventory Released and Shutdown Inventory by Isotope Groups for the Two

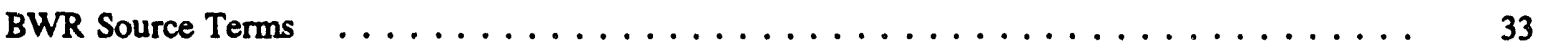

4.2.6 Inventory Released and Shutdown Inventory by Individual Isotope for the

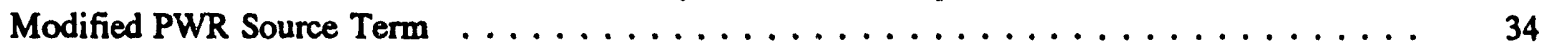

4.2.7 Inventory Released and Shutdown by Isotope Groups for the Modified PWR Source Term . . . . . . . . . . . . . . . . 36

4.2.8 Early Fatalities for the Three Adjusted Source Terms $\ldots \ldots \ldots \ldots \ldots \ldots \ldots \ldots$

4.2.9 Estimated Number of Individuals Receiving Exposure Exceeding Threshold Doses to the three Critical Organs in the Early Fatality Health Effects Model $\ldots \ldots \ldots \ldots \ldots \ldots$ 
4.3.1 Estimated Mean Early Fatalities for the Three Modified Source Terms . . . . . . . . . . . . . . 41

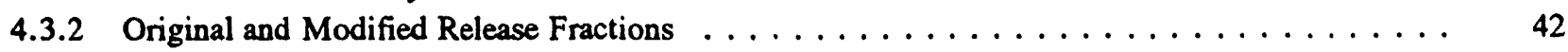

4.3.3 Release Inventories Compared to the Shutdown Core Inventory for the Two BWR Source Terms (Conservative Evacuation Case) $\ldots \ldots \ldots \ldots$

4.3.4 Release Inventory Compared to the Shutdown Core Inventory for the PWR Source Term (Conservative Evacuation Case) $\ldots \ldots \ldots$

4.3.5 Release Inventories Compared to the Shutdown Core Inventory (by Group) for the Two BWR Source Terms (Conservative Evacuation Case) . . . . . . . . . . . . . 48

4.3.6 Release Inventory Compared to the Shutdown Core Inventory (by Group) for the PWR Source Term (Conservative Evacuation Case) . . . . . . . . . . . . . . 48

4.3.7 Estimated Number of Individuals Exposed to Doses Exceeding Fatality Threshold Doses (Conservative Evacuation Case) . . . . . . . . . . . . . . . . . .

4.3.8 Estimated Number of Mean Early Fatalities from Four Adjusted Source Terms: Adjusted to Result in One Early Fatality . . . . . . . . . . . . . . . . . . . . . . 50

4.3.9 The Release Fractions for the Original Four Source Terms . . . . . . . . . . . . . . . . . 51

4.3.10 Inventory Released Compared to the Shutdown Core Inventory for the Two BWR Source Terms (Conservative Evacuation and 10 People per Square Mile) . . . . . . . . 52

4.3.11 Inventory Released Compared to the Shutdown Core Inventory for the Two PWR Source Terms (Conservative Evacuation and 10 People per Square Mile) . . . . . . . 54

4.3.12 Grouped Inventory Released Compared to the Shutdown Core Inventory for the Two BWR Source Terms (Conservative Evacuation and 10 People Per Square Mile) . . . . 56

4.3.13 Grouped Inventory Released Compared to the Shutdown Core. Inventory for the Two PWR Source Terms (Conservative Evacuation and 10 People Per Square Mile) .....

4.4.1 Estimated Number of Mean Early Fatalities from the Three Modified

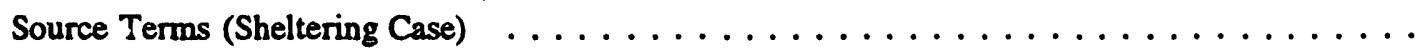

4.4.2 Adjustment of the Release Fractions to Result in One Early Fatality . . . . . . . . . . . . . 60

4.4.3 Inventory Released Compared to the Shutdown Core Inventory for the Two Modified BWR Source Terms ... . . . . . . . . . . . . . . . . . . .

4.4.4 Inventory Released Compared to the Shutdown Core Inventory for the Modified PWR Source Term ............................

4.4.5 Inventory Released Compared to the Shutdown Core Inventory for the Two Modified BWR Source Terms, Listed by Isotope Grouping . . . . . . . . . . . . . . . . .

4.4.6 Inventory Released Compared to the Shutdown Core Inventory for the Modified PWR Source Term, Listed by Isotope Grouping . . . . . . . . . . . . . . . .

4.4.7 The Number of Cases of Exposure Exceeding Threshold to the Three Organs

Used in the Early Fatality Health Effects Model . . . . . . . . . . . . . . . . . . .

4.5.1 Basic Input Assumption for the Equivalent Curies Determination . . . . . . . . . . . . 75

4.5.2 Mean Early Fatalities for I-131 Release: Case 1 . . . . . . . . . . . . . . . . . . . 76

4.5.3 Mean Early Fatalities for I-131 Release: Case 2 . . . . . . . . . . . . . . . . . . . 77

4.5.4 Estimated Population Exceeding Threshold Dose for I-131 Release:

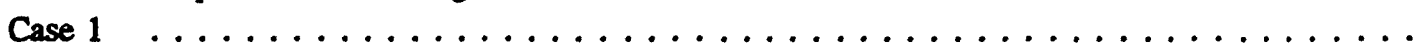

4.5.5 Estimated Population Exceeding Threshold Dose for I-131 Release:

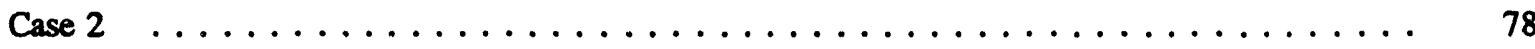

4.5.6 Population Weighted Risk at Site Boundary for I-131 Release: Case 1 . . . . . . . . . . . 79 


\section{List of Tables (Continued)}

4.5.7 Population Weighted Risk at Site Boundary for I-131 Release: Case $2 \ldots \ldots \ldots$

4.5.8 Mean Early Fatalities for I-131 Release with Ten People/Sq. Mi.:

Case 1 Release Timing . . . . . . . . . . . . . . . . . . . . 81

4.5.9 Mean Early Fatalities for I-131 Release with Ten People/Sq. Mi.:

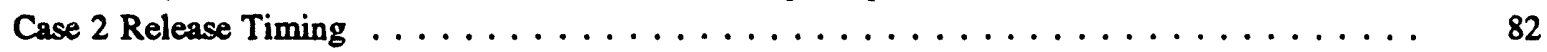

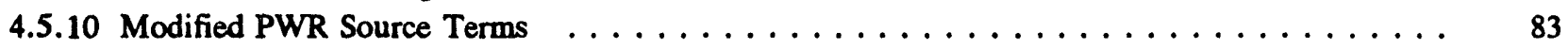

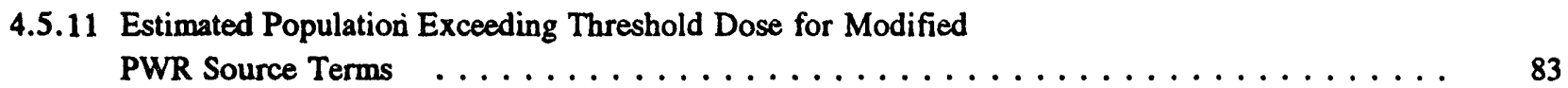

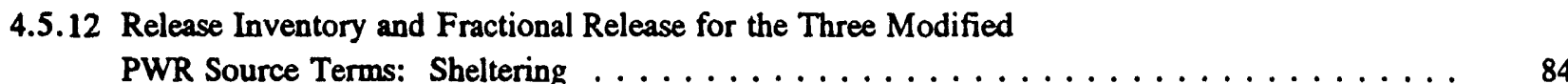

4.5.13 Grouped Release Inventory and Fractional Group Releases for

5.1 Candidate Large Release Source Terms $\ldots \ldots \ldots \ldots \ldots \ldots \ldots \ldots \ldots$

Appendix A

A.1 Characteristics of Surry Release Categories $\ldots \ldots \ldots \ldots \ldots \ldots \ldots \ldots \ldots \ldots \ldots$ A-2

A.2 Characteristics of Sequoyah Release Categories $\ldots \ldots \ldots \ldots \ldots \ldots \ldots \ldots \ldots \ldots$ A-2

A.3 Characteristics of Zion Release Categories $\ldots \ldots \ldots \ldots \ldots \ldots \ldots \ldots \ldots \ldots \ldots \ldots \ldots$

A.4 Characteristics of Peach Bottom Release Categories $\ldots \ldots \ldots \ldots \ldots \ldots \ldots \ldots \ldots$

A.5 Characteristics of Grand Gulf Release Categories $\ldots \ldots \ldots \ldots \ldots \ldots \ldots \ldots \ldots \ldots \ldots$

A.6 Characteristics of LaSalle Release Categories $\ldots \ldots \ldots \ldots \ldots \ldots \ldots \ldots \ldots \ldots \ldots$

A.7 Radionuclide Release Characteristics into Environment for Surry $\ldots \ldots \ldots \ldots \ldots \ldots \ldots$

A.8 Radionuclide Release Characteristics into Environment for Sequoyah $\ldots \ldots \ldots \ldots \ldots \ldots$. . . . .

A.9 Radionuclide Release Characteristics into Environment for Zion . . . . . . . . . . . A-6

A.10 Radionuclide Release Characteristics into Environment for Peach Bottom . . . . . . . . . A-6

A.11 Radionuclide Release Characteristics into Environment for Grand Gulf . . . . . . . . . . . . A-7

A.12 Radionuclide Release Characteristics into Environment for LaSalle . . . . . . . . . . A A-8

Appendix B

B.2.1 Centerline Dose (Rem) to Red Marrow as a Function of Distance by

Pathway for the Source Term MPB1 ....................

B.2.2 Centerline Dose (Rem) to Lungs as a Function of Distance by Pathway

for the Source Term MPB1 $\ldots \ldots \ldots \ldots \ldots \ldots \ldots \ldots \ldots \ldots \ldots$

B.2.3 Centerline Dose (Rem) to Lower Large Intestine as a Function of Distance by

Pathway for the Source Term MPB1 $\ldots \ldots \ldots \ldots \ldots \ldots \ldots \ldots \ldots \ldots$

B.2.4 Fraction of the Centerline Dose to the Red Marrow as a Function of Distance

and by Pathway for the Total Population and for Each Cohort for the Source Term MPB1 $\quad \ldots \quad$ B-8

B.2.5 Fraction of the Centerline Dose to the Lungs as a Function of Distance and by Pathway for the Total Population and for Each Cohort for the Source Term MPB1 . . . . B-9

B.2.6 Fraction of the Centerline Dose to the Lower Large Intestine as a Function of Distance and by Pathway for the Total Population and for Each Cohort for the Source Term MPB1 . . B-10 


\section{List of Tables (Continued)}

Page

B.3.1 Centerline Dose (Rem) to the Red Marrow as a Function of Distance by Pathway for

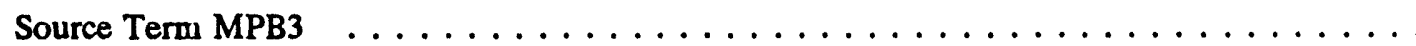

B-1:

B.3.2 Centerline Dose (Rem) to the Lungs as a Function of Distance by Pathway for

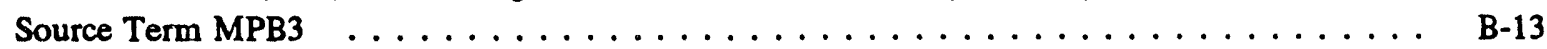

B.3.3 Centerline Dose (Rem) to the Lower Large Intestine as a Function of Distance by Pathway for Source Term MPB3 $\ldots \ldots \ldots \ldots \ldots \ldots \ldots \ldots \ldots \ldots \ldots \ldots$ B-15

B.3.4 Fraction of the Centerline Dose to the Red Marrow as a Function of Distance and by Pathway for the Total Population, the Evacuees and the Non-evacuees

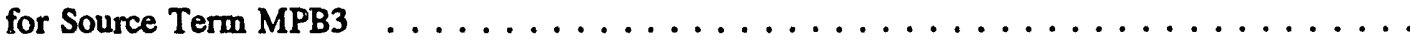

B.3.5 Fraction of the Centerline Dose to the Lungs as a Function of Distance and by Pathway for the Total Population, the Evacuees, and the Non-evacuees for Source Term MPB3 . . . . .

B.3.6 Fraction of the Centerline Dose to the Lower Large Intestine as a Function of Distance and by Pathway for the Total Population, the Evacuees, and the Non-evacuees for Source Term MPB3

B.4.1 Centerline Dose (Rem) to Red Marrow as a Function of Distance by Pathway

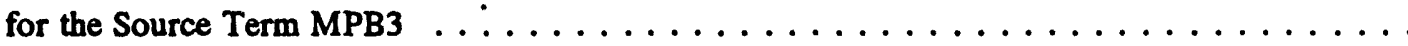

B.4.2 Centerline Dose (Rem) to Lungs as a Function of Distance by Pathway for

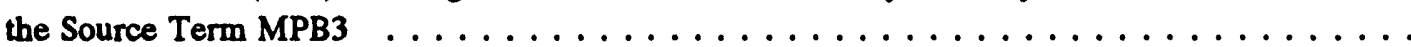

B.4.3 Centerline Dose (Rem) to the Lower Large Intestine as a Function of Distance by Pathway for the Source Term MPB3 $\ldots \ldots \ldots \ldots \ldots \ldots \ldots \ldots \ldots$

B.4.4 Fraction of the Centerline Dose to the Red Marrow as a Function of Distance and by Pathway for the Total Population and for Each Cohort for Source Term MPB3 . . . . .

B.4.5 Fraction of the Centerline Dose to the Lungs as a Function of Distance and by Pathway for the Total Population and for Each Cohort for Source Term MPB3

B.4.6 Fraction of the Centerline Dose to the Lower Large Intestine as a Function of Distance and by Pathway for the Total Population and for Each Cohort for Source Term MPB3

B.5.1 Dose to the Three Critical Organs by Pathway as a Function of Distance to Produce

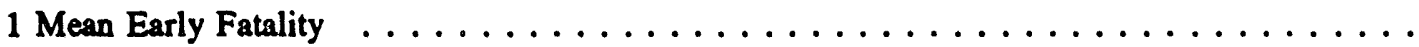

B.5.2 Dose (Rem) as a Function of Distance to the Three Critical Organs by Pathway for the Modified PWR Source Terms That Result in One Early Fatality . . . . . . . . . . . . .

B.5.3 Fraction of Dose as a Function of Distance to the Three Critical Organs

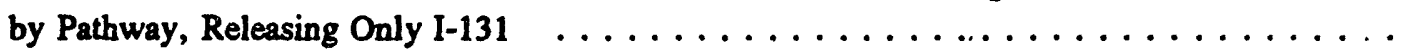

B.5.4 Fraction of Dose as a Function of Distance to the Three Critical Organs by Pathway for Modified PWR Source Terms 


\section{EXECUTIVE SUMMARY}

The Nuclear Regulatory Commission has stated a hierarchy of safety goals: the qualitative safety goals as Level I of the hierarchy; the quantitative health objectives as Level II and the contemplated large release guideline as Level III. The large release guideline has been stated in qualitative terms as a magnitude of release of the core inventory whose frequency should not exceed $10^{-6}$ per reactor year. However the Commission did not provide a quantitative specification of a large release. One recommendation was made that a large release be defined as having the potential for causing an off-site early fatality within one mile of the site to the average individual. This report focuses on an examination of releases which have a potential to lead to one early fatality.

The basic information required to set up the calculations was derived from the simplified source terms which were obtained from approximations of the NUREG-1150 source terms. Since the calculation of consequences is affected by a large number of assumptions, a generic site with a population density and meteorology was specified. At this site, various emergency responses (including no response) were assumed based on information derived from earlier studies. For each of the emergency response assumptions, sets of calculations were performed with the simplified source terms. These included adjustments to the source terms, such as the timing of the release, the core inventory, and the release fractions of different radionuclides, to have a potential to lead to one mean early fatality in each case. Each of the source terms, so defined, has the potential to be a candidate for a large release. 


\section{ACKNOWLEDGEMENTS}

We would like to thank T. King, L. Soffer, J. Ridgely and C. Ader of the U. S. Nuclear Regulatory Commission for their comments on this work, C. Conrad for assistance with the calculations and B. Kponou, K. Ryan, and B. Bovell for the production of this report. 


\section{INTRODUCTION}

The Nuclear Regulatory Commission (NRC) issued a Policy Statement regarding the establishment of Safety Goals for the Operations of Nuclear Power Plants which defined, in broad terms, acceptable levels of radiological risk to the public [1]. The Commission enunciated two qualitative safety goals, one for individual risk and the other for societal risk. These qualitative safety goals were implemented by two Quantitative Health Objectives (QHOs) which set numerical goals for the individual risk of early fatalities and the risk of latent cancers.

As an aid to the implementation of the safety goals in the regulatory process, the Commission proposed for further examination the following general plant performance guideline:

"...the overall mean frequency of a large release of radioactive materials to the environment from a reactor accident should be less than 1 in 1,000,000 per year of reactor operation." [1]

\subsection{Large Release Definition}

The notion of a "large release" implies the existence of a threshold in release space; that is, given a spectrum of possible releases there exists a boundary which distinguishes "large" from "not large". Attempts have been made to define a large release magnitude based on off-site health effects. There is an inherent arbitrariness in their definition since off-site health effects depend not only on release magnitude but also on site-specific parameters, such as population. Thus what would be a large release at one site, would not necessarily be one at some other site. Weather variability and wind rose are other site-specific factors. In a hypothetical calculation (reported in Section 4.5) the population was fixed by assuming one person located at the site boundary in each of the 16 angular sectors around the site and the population weighted risk of early fatalities was calculated for large releases. The population weighted risk of early fatality is the number of early fatalities (which is one in this case since for a particular release the wind only blows into one angular sector) divided by the total population "at risk" which is 16 in this particular case. Only at 95 th percentile weather (extreme weather) and very large releases did the population weighted risk exceed $1 / 16$. This report explores the implications of some potential definitions.

In the past, the NRC staff has considered several alternate definitions of a large release. These include:

1) A collection of all releases that would result in one or more early fatalities;

2) The use of an individual dose definition of large release;

3) A definition of a "large release" source term in the traditional form of a fractional release of the core inventory of various radionuclide groups to the environment, the timing of the release, etc.;

4) Any release from an event that involves severe core damage, primary system pressure boundary failure, and early containment failure.

These alternatives, as well as a discussion of their potential advantages and disadvantages, were described in SECY89-102 [2].

This study explores the specification of a large release source term which has the potential to result in one eariy off-site fatality within one mile of the plant boundary. The large release study was closely coupled with another effort to rebaseline the technical basis for nuclear power station siting [3]. In the siting study, sets of simplified source terms were developed for the five NUREG-1150 plants [4] and for LaSalle [5]. The consequences arising from these sets of source terms were examined at a series of generic sites, that varied in population density, weather 


\section{Introduction}

characteristics, and exclusion boundary distance. The selection of parameters, ranges and distributions was based on the choice of an "80th percentile" site from the viewpoint of consequences when compared with existing nuclear reactor sites in the U.S. These sets of alternative sites, meteorologies, and simplified source terms provided a spectrum from which specific selections were made to examine the quantification of a large release source term. The analytical strategy adopted was to select a generic site and a meteorology that would ensure conservative estimates of consequences for a given source term. The other factors were varied by selection or adjustment to result in the potential to lead to one mean early fatality. 


\section{FACTORS AFFECTING EARLY FATALITY CALCULATION}

There are numerous factors which influence the calculation of early fatalities: the physical characteristics of the source term, the nature of emergency response taken to mitigate accident consequences, site demographics, and site meteorology, etc. Figure 2.1 shows three primary factors: release magnitude, release timing, and emergency response, along three axes. With site demographics, meteorology, etc. fixed, various combinations of values of the depicted variables, for example, low magnitude/early timing/conservative (slow) emergency response, or, alternatively, large magnitude/late timing/NUREG-1150 type (prompt) response, could give rise to one early fatality. This schematic depiction is simply meant to show that there is no unique answer (a single point in the parameter space) to the question of what source term leads to one early fatality. There are, instead, a number of possible answers, or sets of points whose locus would define a hypothetical early fatality surface in the schematic parameter space of Fig. 2.1.

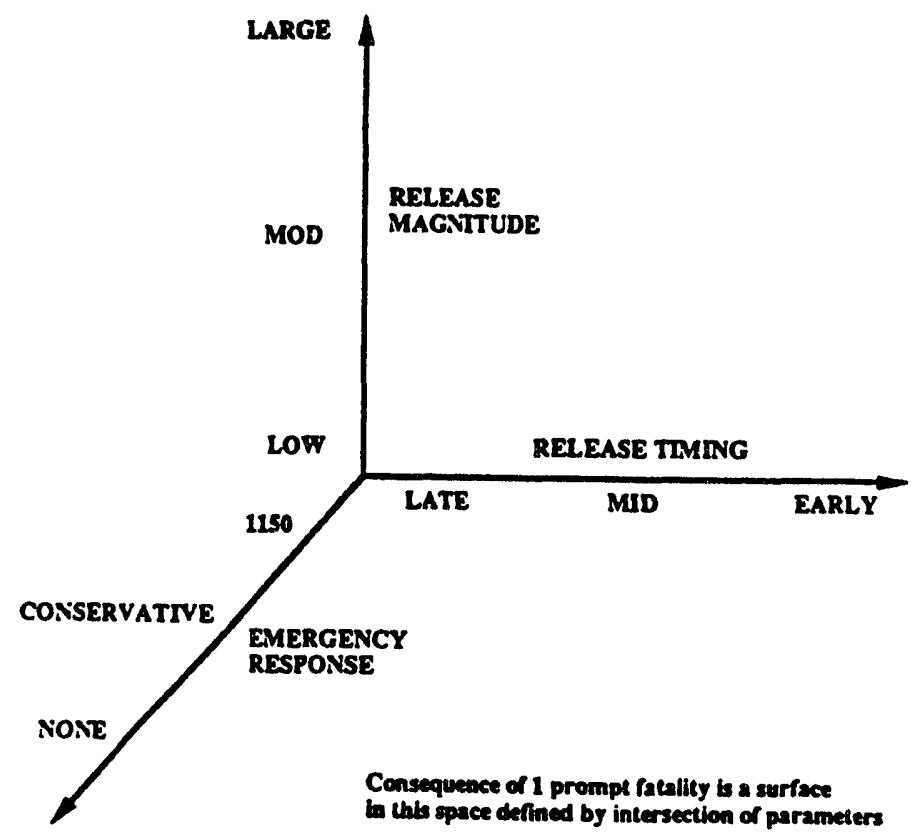

Figure 2.1 Early Fatality Parameter Space

The source term, as traditionally specified by a set of fractional releases of the initial core inventory, and the time of release to the environment, defines the magnitude of the release to the environment. The timing is significant in that physical removal processes occurring in containment can lower the fractional releases and radioactive decay can significantly lower the amounts of short lived isotopes which reach the environment and which are known to contribute significantly to the early dose and therefore early fatalities. On the other hand, timely and effective evacuation can in many instances mitigate early consequences which in the absence of such emergency actions would be quite serious. Hence, it is to be expected that a spectrum of source terms exists, given their magnitude and timing, and given the type and effectiveness of emergency responses that could occur, which will result in the potential to lead to one mean early fatality and could be candidates for a large release source term definition. 


\subsection{Emergency Protective Actions}

The MELCOR Accident Consequences Code System (MACCS) [6] was used to calculate off-site early fatalities. The protective actions are modeled in MACCS. The emergency responses modeled in the EARLY module of the MACCS code are of importance. Three emergency responses are evacuation, sheltering, or no action (or "No Evacuation") for the population that does not participate in the emergency actions.

\subsubsection{Evacuation}

Three emergency protective (EP) schemes were primarily considered:

(1) "Mean EP": This case was based on the mean of the evacuation times and speeds determined for the five NUREG-1150 sites. The delay from waming to the start of evacuation was 1.9 hours, and the evacuation speed was 5.9 miles per hour $(2.6 \mathrm{~m} / \mathrm{sec})$. The evacuation was out to $10 \mathrm{miles}$, and had a $99.5 \%$ participation, consistent with the NUREG-1150 study;

(2) "Conservative EP": This case assumed a slower and later evacuation than the Mean EP case. The assumptions were a 6 hour delay from the warning to start of evacuation and an evacuation speed of 2.5 miles per hour $(1.1 \mathrm{~m} / \mathrm{sec})$, based on the analysis of Hans and Sell [7]. Evacuation was out to 10 miles and the participation was $95 \%$;

(3) "No Evacuation": This case assumed that no evacuation occurred and that people in the affected area went about their normal activities. This is clearly an unlikely response, but the case should bound the maximum consequences associated with a given release.

The table below shows the values of the selected parameters in the mean and conservative EP cases.

\section{Table 2.1.1 EP Parameters}

\begin{tabular}{|l|c|c|c|}
\hline \multicolumn{1}{|c|}{ Parameter } & Mean EP Case & $\begin{array}{c}\text { Conservative } \\
\text { EP Case }\end{array}$ & $\begin{array}{c}\text { Sheltering } \\
\text { Case }\end{array}$ \\
\hline Evacuation Speed (miles per hour) & 5.9 & 2.5 & - \\
\hline Delay Time & $1.9 \mathrm{hrs}$ & $6 \mathrm{hrs}$ & $12 \mathrm{hrs}$ \\
\hline \% Participation & $99.5 \%$ & $95 \%$ & $95 \%$ \\
\hline
\end{tabular}

\subsubsection{Sheltering Scheme}

An alternative to immediate evacuation is sheltering of the people in building structures during the passage of the release plume, followed by relocation. There are some outlying cases where an early evacuation can actually increase the number of consequences if the timings of the release and of the evacuation are close. This causes the 
evacuees to travel with the radioactive plume. In this study, nly one sheltering scheme was examined. The population within 10 miles of the exclusion boundary was sheltered for $12 \mathrm{hrs}$, then relocated. A $95 \%$ participation rate was assumed. Additionally, some sheltering credit is given in the code as part of "normal activity" to those who do not evacuate and some credit is also given to those participating in the emergency procedure after the release had started but before the evacuees started to move.

Table 2.1.2 shows the values of the shielding and sheltering dose reduction parameters for different pathways for the evacuees (while in the process of evacuation), those continuing normal activity and those taking shelter, respectively.

Table 2.1.2 Shielding and Sheltering Dose Reduction Parameters (based on average of five plants in NUREG-1150)

\begin{tabular}{|c|c|c|c|}
\hline & Cloud & Ground & Skin \\
\hline Evacuees & 1.0 & 0.5 & 1.0 \\
\hline Normal & 0.75 & 0.33 & 0.41 \\
\hline Sheltered & 0.6 & 0.2 & 0.33 \\
\hline
\end{tabular}

\subsection{Source Terms}

In this study the six sets of simplified source terms developed in Ref. 3 were examined to identify candidate large release source terms. A series of tables in Appendix A provides a description (in terms of the major accident progression characteristics) of the accident sequences selected and the physical characteristics of the associated accident source terms.

In general, for a given emergency protective response, e.g., Mean EP assumptions, a survey calculation identified a subset of three or four source terms which resulted in the potential to lead to one mean early fatality. These source terms were then adjusted in some fashion until the MACCS code predicted one early fatality. The adjustments were to the release timing, reactor power (i.e., initial core inventory) or to the actual fractional releases. Details of these adjustments are provided in Section 4. 
2 Factors Affecting Early Fatality Calculation 


\section{SITE PARAMETERS}

Four generic sites were investigated. These sites were developed in Reference 3. The demographic assumptions for these sites were linked to population density guidance in Regulatory Guide 4.7 [8]. With the exception of Case 2 the population distributions were uniform from the exclusion boundary out to 30 miles. Details of the population distribution beyond 30 miles may be found in Reference 3, but are not important to this study since early fatalities generally occur within a few miles of the release point. Additionally, the large release source term candidates explored in this study are those source terms that result in a prediction of one mean early fatality within one mile of the site boundary. Furthermore, the quantitative health objective (QHO) for early fatalities is defined in terms of a bound on the population weighted risk of early fatality for the average individual within one mile of the site boundary. It is shown later in the study that a large release guideline of a frequency of $10^{-6}$ per year of an early fatality within one mile of the site boundary is more conservative than the QHO. The assumed population densities are 500, 1000 and 2000 people per square mile for Cases 1, 5 and 7, respectively. For Case 2, the population density is 100 people per square mile with a city of 125,000 people between 5 and 20 miles of the generic plant.

Regulatory Guide 4.7 states that, if the population density projected at the time of initial operation of a nuclear power station exceeds 500 people per square mile averaged over any radial distance out to 30 miles (cumulative population at a distance divided by the area at that distance), or the projected population density over the lifetime of the facility exceeds 1000 people per square mile, consideration should be given to alternative sites with lower population densities.

Hence, two cases represent the densities cited in Regulatory Guide 4.7, while the 2000 people per square mile case represents a conservative extension of Regulatory Guide 4.7 densities. In Case 2, a small city of 125,000 people is located basically in one $22.5^{\circ}$ sector, in the region from 5 to 10 miles beyond the plant. The surrounding population density was 100 people per square mile. This case is also consistent with the start of the plant life population density given in Regulatory Guide 4.7.

The majority of the calculations were performed with an exclusion area boundary radius of 0.25 mile, which can be viewed as a conservative assumption since the Regulatory Guide 4.7 recommended minimum exclusion area boundary distance of 0.4 mile. The exclusion area boundary radius was 0.4 mile for Case 6 , and this case was used for developing candidates for the large release source term.

Two scoping series of probabilistic consequence assessment calculations were performed with the MACCS code. These were for the no evacuation and the mean evacuation cases. All six sets of the simplified source terms were examined for the purpose of screening candidate source terms for the large release definition. These results are presented in Section 4.1 and 4.2 .

This type of scoping calculation was not repeated for the conservative evacuation or for the shelter responses. Candidates for these latter cases were identified by inspection of results of the former cases.

\subsection{Meteorology}

The selection of meteorology (weather data) employed in this study was developed in Reference 3. In an attempt to provide reasonably conservative estimates of accident consequences, a bounding meteorology was selected. The criteria for the selection of the bounding meteorology was the amount of rainfall, the frequency of high stability weather classes and low wind speed. 


\section{Site Parameters}

The selected meteorology is bounding in the sense that the predicted accident consequences would equal or exceed those at a spectrum of reactor sites at the 80th percentile. A statistical analysis of data from 29 National Weather Service (NWS) sites was used to establish the 80 th percentile values based on the attributes of weather important to consequences. This analysis identified Charleston, South Carolina as the bounding weather site in terms of wind speed, stability and rain data. These data were then composited with an independently determined 80th percentile mixing height (based on the NWS data) and an 80th percentile windrose (based upon data from $\sim 79$ actual reactor sites). 


\section{RESULTS}

In an effort to examine a large release source term definition for severe nuclear power plant accidents, a series of probabilistic consequence calculations were made with the MACCS code [6]. In this section we present results of these calculations. The calculations covered a range of assumptions, and identify sets of source terms that will have the potential to lead to one early fatality. The consequence calculations are very dependent on the type of emergency response assumed in relation to the physical characteristics of the source term. Four sets of source terms were developed. These are associated with the following emergency responses: no evacuation, mean evacuation, conservative evacuation, and sheltering followed by relocation. In each of these cases, candidate source terms were identified from the six sets of simplified source terms. The selection criterion was the proximity to the potential to lead to one mean early fatality. The candidate source terms were then adjusted by various arbitrary means to effect the potential to lead to one mean early fatality. A fifth series of calculations, that addressed a large release source term definition as an equivalent release of iodine-131, is provided in the last subsection. In general, the accidental releases of radionuclides from containment are compared to the shutdown core inventory of radionuclides present in a $3800 \mathrm{MWth}$ plant, (see Tables 4.0.1 and 4.0.2).

The candidate source terms that are developed for the definition of large release started with the simplified source terms presented in Reference 3. These simplified source terms were developed from the NUREG-1150 [4] study of five nuclear power plants and LaSalle [5]. Following the notation in Reference 3, these source terms are denoted as:

$$
R x x x y
$$

where $x x x$ signifies the power plant from which they originated, and $y$ is the source term number. $x x x$ can be:

$\begin{array}{ll}\text { GG } & \text { Grand Gulf } \\ \text { LAS } & \text { LaSalle } \\ \text { PB } & \text { Peach Bottom } \\ \text { SEQ } & \text { Sequoyah } \\ \text { SUR } & \text { Surry } \\ \text { Z } & \text { Zion }\end{array}$

In this report some of these source terms were modified until MACCS predicted one mean early fatality. For these cases, the modified source term is preceded with an " $M$ " instead of an " $R$ ".

\subsection{Source Terms for the No Evacuation Case}

The development of source terms that have the potential to lead to one early fatality was initially performed by adjusting selected source terms until one early fatality was predicted by MACCS. The candidate source terms were selected by choosing 3-5 source terms that resulted in a MACCS prediction of approximately one early fatality. Table 4.1.1 shows the MACCS estimated number of early fatalities for the no evacuation case for the four different population distributions and for the exclusion zone distance of 0.25 mile. One exception is Case 6 where the exclusion area distance is 0.4 mile. These calculations were for the MACCS estimated number of early fatalities one mile beyond the exclusion area distance, the specified distance for which the NRC's quantitative health objective for early fatalities is calculated. 
The distributions that are calculated in the MACCS code, the Complementary Cumulative Distribution Functions (CCDFs), represent the probability of exceeding a particular consequence. These probabilities are, in turn, dependent on the particular weather sequences. Therefore a distribution of probabilities, the CCDF, is developed taking into account the different weather conditions encountered. The relationship of the CCDF to the actual probability function is then:

$$
\operatorname{CCDF}(X)=\int_{X}^{\infty} p(x) d x
$$

When the code calculates mean values below 0.1 there may not be any weather sequence(s) that resulted in a predicted value of one or more early fatalities. These cases, denoted by $<0.1$, mean that the MACCS code calculated a small, but non-zero probability of a fatality. The number 0 is inserted in these tables wherever the MACCS code calculated zero probability of any early fatalities.

For the no evacuation case using population Case 6 (1000 people per square mile and an exclusion boundary of 0.4 mile), the candidate source terms selected for modification were RGG3, RLAS3, RLAS6, and RZ2. To modify the source terms to result in a prediction of one early fatality, the time of release for RGG3, RLAS3, and RZ2 was decreased, while the power level for RLAS6 decreased. The relative release fractions for all source terms were retained. Reducing the release time increases the released activity, since less decay occurs prior to release. The modifications that were made are summarized in Table 4.1.2. Table 4.1.3 shows the number of mean early fatalities estimated by MACCS that are predicted after the scaling. All of the modified source terms lead to approximately one early fatality within one mile (with a small contribution, in some cases, beyond one mile) of the exclusion boundary. For one early fatality within one mile the population weighted risk by definition is $1 / \mathrm{POP1}$ where POP1 is the total population out to one mile from the exclusion boundary. For a density of $1000 / \mathrm{sq}$. mi, the population weighted risk in this instance is about $2 \times 10^{-4}$.

If one associates the candidate large release accident frequency of $10^{-6} / \mathrm{yr}$ with the individual probability of fatality, one obtains $2 \times 10^{-4}$ times $10^{-6} / \mathrm{yr}$ which equals $2 \times 10^{-10} / \mathrm{yr}$. This value, when compared to the total risk represented by the early fatality quantitative health objective $\left(5 \times 10^{-7} / \mathrm{yr}\right)$ is seen to be several orders of magnitude lower. Based on current understanding of severe accident source terms, (e.g., NUREG-1150) the large release guideline, examined in this study, represents a more stringent plant performance objective than the early fatality quantitative health objective.

The actual invintory released to the environment depends on the initial core inventory, the fractional releases, and the time of release to the environment. Adjustment of the reactor power linearly effects the initial core inventory, while an adjustment of the release timing changes the radioactive decay correction.

Tables 4.1.4 and 4.1.5 show the actual releases (in Curies) and fractions of the shutdown inventories for the three BWR source terms after scaling in order to produce the potential to lead to one early fatality. These numbers have not been weighted by the isotopes' dose conversion factors. Tables 4.1 .6 and 4.1 .7 provide the activities of the released radioisotopes and their relative release fractions, within the traditional radioisotope grouping scheme. Tables 4.1.8 and 4.1.9 provide similar results for the modified PWR source term.

A detailed examination of the dose pathways that may contribute to fatality, indicated that early fatality in the no evacuation case was dominated by the seven day groundshine dose calculated by the MACCS code. The magnitudes of these adjusted source terms present little inhalation and cloudshine hazard. The value of this set of no evacuation large release source terms is to establish a lower bound for further considerations. 
Table 4.0.1 Default (3578 MWth) and Adjusted* (3800 MWth) Shutdown Core Inventory for a BWR Reactor

\begin{tabular}{|c|c|c|c|}
\hline Isotope & Group & Default Inventory (Ci) & Adjusted Inventory (Ci) \\
\hline KR-85 & 1 & $8.96 \mathrm{E}+05$ & $9.52 \mathrm{E}+05$ \\
\hline KR-85M & 1 & $3.26 \mathrm{E}+07$ & $3.46 \mathrm{E}+07$ \\
\hline KR-87 & 1 & $5.93 E+07$ & $6.29 \mathrm{E}+07$ \\
\hline KR-88 & 1 & $8.00 E+07$ & $8.50 \mathrm{E}+07$ \\
\hline $\mathrm{XE}-133$ & 1 & $1.94 \mathrm{E}+08$ & $2.06 \mathrm{E}+08$ \\
\hline $\mathrm{XE}-135$ & 1 & $4.61 E+07$ & $4.90 \mathrm{E}+07$ \\
\hline $\mathrm{I}-131$ & 2 & $9.24 \mathrm{E}+07$ & $9.81 E+07$ \\
\hline $1-132$ & 2 & $1.36 \mathrm{E}+08$ & $1.44 \mathrm{E}+08$ \\
\hline I-133 & 2 & $1.94 \mathrm{E}+08$ & $2.06 E+08$ \\
\hline I-134 & 2 & $2.12 E+08$ & $2.25 E+08$ \\
\hline I-135 & 2 & $1.82 E+08$ & $1.94 \mathrm{E}+08$ \\
\hline CS-134 & 3 & $1.51 E+07$ & $1.61 \mathrm{E}+07$ \\
\hline CS-136 & 3 & $4.06 E+06$ & $4.31 E+06$ \\
\hline CS-137 & 3 & $9.05 \mathrm{E}+06$ & $9.62 E+06$ \\
\hline RB-86 & 3 & $5.02 \mathrm{E}+04$ & $5.33 E+04$ \\
\hline SB-127 & 4 & $8.32 E+06$ & $8.83 E+06$ \\
\hline SB-129 & 4 & $2.89 E+07$ & $3.07 \mathrm{E}+07$ \\
\hline TE-127 & 4 & $8.05 E+06$ & $8.55 E+06$ \\
\hline TE-127M & 4 & $1.08 E+06$ & $1.15 E+06$ \\
\hline TE-129 & 4 & $2.71 E+07$ & $2.88 \mathrm{E}+07$ \\
\hline TE-129M & 4 & $7.12 E+06$ & $7.56 \mathrm{E}+06$ \\
\hline TE-131M & 4 & $1.37 \mathrm{E}+07$ & $1.45 \mathrm{E}+07$ \\
\hline TE-132 & 4 & $1.34 \mathrm{E}+08$ & $1.42 E+08$ \\
\hline SR-89 & 5 & $9.93 E+07$ & $1.05 E+08$ \\
\hline SR-90 & 5 & $7.02 E+06$ & $7.46 \mathrm{E}+06$ \\
\hline SR-91 & 5 & $1.29 E+08$ & $1.37 \mathrm{E}+08$ \\
\hline SR-92 & 5 & $1.35 \mathrm{E}+08$ & $1.43 E+08$ \\
\hline CO-58 & 6 & $5.47 \mathrm{E}+05$ & $5.81 E+05$ \\
\hline$C 0-60$ & 6 & $6.55 \mathrm{E}+05$ & $6.95 E+05$ \\
\hline MO-99 & 6 & $1.74 E+08$ & $1.85 \mathrm{E}+08$ \\
\hline
\end{tabular}


Table 4.0.1 (Continued)

\begin{tabular}{|c|c|c|c|}
\hline Isotope & Group & Default Inventory (Ci) & Adjusted Inventory (Ci) \\
\hline RH-105 & 6 & $6.56 E+07$ & $6.97 \mathrm{E}+07$ \\
\hline RU-103 & 6 & $1.32 \mathrm{E}+08$ & $1.40 \mathrm{E}+08$ \\
\hline RU-105 & 6 & $8.79 E+07$ & $9.34 \mathrm{E}+07$ \\
\hline RU-106 & 6 & $3.59 \mathrm{E}+07$ & $3.81 \mathrm{E}+07$ \\
\hline TC-99M & 6 & $1.50 \mathrm{E}+08$ & $1.59 E+08$ \\
\hline AM-241 & 7 & $7.85 E+03$ & $8.33 E+03$ \\
\hline CM-242 & 7 & $2.07 \mathrm{E}+06$ & $2.20 \mathrm{E}+06$ \\
\hline CM-244 & 7 & $1.12 \mathrm{E}+05$ & $1.19 \mathrm{E}+05$ \\
\hline LA-140 & 7 & $1.80 \mathrm{E}+08$ & $1.91 \mathrm{E}+08$ \\
\hline LA-141 & 7 & $1.66 \mathrm{E}+08$ & $1.76 \mathrm{E}+08$ \\
\hline LA-142 & 7 & $1.60 \mathrm{E}+08$ & $1.70 \mathrm{E}+08$ \\
\hline NB-95 & 7 & $1.51 \mathrm{E}+08$ & $1.60 \mathrm{E}+08$ \\
\hline ND-147 & 7 & $6.82 \mathrm{E}+07$ & $7.24 \mathrm{E}+07$ \\
\hline PR-143 & 7 & $1.53 \mathrm{E}+08$ & $1.62 E+08$ \\
\hline$Y-90$ & 7 & $7.52 \mathrm{E}+06$ & $7.99 \mathrm{E}+06$ \\
\hline $\mathrm{Y}-91$ & 7 & $1.21 \mathrm{E}+08$ & $1.29 E+08$ \\
\hline $\mathrm{Y}-92$ & 7 & $1.35 \mathrm{E}+08$ & $1.44 \mathrm{E}+08$ \\
\hline$Y-93$ & 7 & $1.54 \mathrm{E}+08$ & $1.63 E+08$ \\
\hline ZR-95 & 7 & $1.59 \mathrm{E}+08$ & $1.69 E+08$ \\
\hline ZR-97 & 7 & $1.64 \mathrm{E}+08$ & $1.74 \mathrm{E}+08$ \\
\hline CE-141 & 8 & $1.60 E+08$ & $1.70 E+08$ \\
\hline CE-143 & 8 & $1.56 \mathrm{E}+08$ & $1.65 E+08$ \\
\hline CE-144 & 8 & $1.04 E+08$ & $1.10 E+08$ \\
\hline NP-239 & 8 & $2.03 E+09$ & $2.16 \mathrm{E}+09$ \\
\hline PU-238 & 8 & $1.41 \mathrm{E}+05$ & $1.50 \mathrm{E}+05$ \\
\hline PU-239 & 8 & $3.58 \mathrm{E}+04$ & $3.80 E+04$ \\
\hline PU-240 & 8 & $4.48 E+0 i$ & $4.76 E+04$ \\
\hline PU-241 & 8 & $7.72 \mathrm{E}+06$ & $8.20 E+06$ \\
\hline BA-139 & 9 & $1.79 E+08$ & $1.90 E+08$ \\
\hline BA-140 & 9 & $1.76 E+08$ & $1.87 \mathrm{E}+08$ \\
\hline
\end{tabular}

*1.062 is factor used for adjustment of the MACCS default BWR core inventory. 
Table 4.0.2 Default (3412 MWth) and Adjusted* (3800 MWth) Shutdown Core Inventory for a PWR Reactor

\begin{tabular}{|c|c|c|c|}
\hline Isotope & Group & Default Inventory (Ci) & Adjusted Inventory (Ci) \\
\hline KR-85 & 1 & $6.69 E+05$ & $7.45 E+05$ \\
\hline KR-85M & 1 & $3.13 \mathrm{E}+07$ & $3.49 \mathrm{E}+07$ \\
\hline KR-87 & 1 & $5.72 \mathrm{E}+07$ & $6.38 \mathrm{E}+07$ \\
\hline KR-88 & 1 & $7.74 \mathrm{E}+07$ & $8.62 E+07$ \\
\hline XE-133 & 1 & $1.83 E+08$ & $2.04 E+08$ \\
\hline $\mathrm{XE}-135$ & 1 & $3.44 \mathrm{E}+07$ & $3.83 E+07$ \\
\hline $1-131$ & 2 & $8.66 \mathrm{E} i+07$ & $9.65 \mathrm{E}+07$ \\
\hline I-132 & 2 & $1.28 \mathrm{E}+08$ & $1.42 E+08$ \\
\hline I-133 & 2 & $1.83 E+08$ & $2.04 E+08$ \\
\hline I-134 & 2 & $2.01 E+08$ & $2.24 E+08$ \\
\hline I-135 & 2 & $1.73 E+08$ & $1.92 \mathrm{E}+08$ \\
\hline CS-134 & 3 & $1.17 \mathrm{E}+07$ & i. $30 \mathrm{E}+07$ \\
\hline CS-136 & 3 & $3.56 \mathrm{E}+06$ & $3.96 \mathrm{E}+06$ \\
\hline CS-137 & 3 & $6.53 E+06$ & $7.28 \mathrm{E}+06$ \\
\hline RB-86 & 3 & $5.10 \mathrm{E}+04$ & $5.68 \mathrm{E}+04$ \\
\hline SB-127 & 4 & $7.53 E+06$ & $8.39 E+06$ \\
\hline SB-129 & 4 & $2.67 \mathrm{E}+07$ & $2.97 \mathrm{E}+07$ \\
\hline TE-127 & 4 & $7.28 \mathrm{E}+06$ & $8.11 E+06$ \\
\hline TE-127M & 4 & $9.63 E+05$ & $1.07 \mathrm{E}+06$ \\
\hline TE-129 & 4 & $2.50 \mathrm{E}+07$ & $2.79 E+07$ \\
\hline TE-129M & 4 & $6.60 \mathrm{E}+06$ & $7.36 \mathrm{E}+06$ \\
\hline TE-131M & 4 & $1.26 \mathrm{E}+07$ & $1.41 E+07$ \\
\hline TE-132 & 4 & $1.26 \mathrm{E}+08$ & $1.40 E+08$ \\
\hline SR-89 & 5 & $9.70 \mathrm{E}+07$ & $1.08 \mathrm{E}+08$ \\
\hline SR-90 & 5 & $5.24 \mathrm{E}+06$ & $5.83 E+06$ \\
\hline SR-91 & 5 & $1.25 E+08$ & $1.39 E+08$ \\
\hline SR-92 & 5 & $1.30 \mathrm{E}+08$ & $1.45 E+08$ \\
\hline CO-58 & 6 & $8.71 E+05$ & $9.70 E+05$ \\
\hline $\mathrm{CO}-60$ & 6 & $6.66 \mathrm{E}+05$ & $7.42 \mathrm{E}+05$ \\
\hline MO-99 & 6 & $1.65 E+08$ & $1.84 \mathrm{E}+08$ \\
\hline
\end{tabular}


Table 4.0.2 (Continued)

\begin{tabular}{|c|c|c|c|}
\hline Isotope & Group & Default Inventory $(\mathbf{C} \mathbf{i})$ & Adjusted Inventory (Ci) \\
\hline RH-105 & 6 & $5.53 \mathrm{E}+07$ & $6.16 \mathrm{E}+07$ \\
\hline RU-103 & 6 & $1.23 E+08$ & $1.37 \mathrm{E}+08$ \\
\hline RU-105 & 6 & $7.98 \mathrm{E}+07$ & $8.89 E+07$ \\
\hline RU-106 & 6 & $2.79 E+07$ & $3.11 E+07$ \\
\hline TC-99M & 6 & $1.42 E+08$ & $1.58 E+08$ \\
\hline AM-241 & 7 & $3.13 E+03$ & $3.49 E+03$ \\
\hline $\mathrm{CM}-242$ & 7 & $1.20 \mathrm{E}+06$ & $1.34 \mathrm{E}+06$ \\
\hline CM-244 & 7 & $7.02 E+04$ & $7.82 E+04$ \\
\hline LA-140 & 7 & $1.72 E+08$ & $1.91 E+08$ \\
\hline LA-141 & 7 & $1.57 E+08$ & $1.75 E+08$ \\
\hline LA-142 & 7 & $1.52 E+08$ & $1.69 E+08$ \\
\hline NB-95 & 7 & $1.41 \mathrm{E}+08$ & $1.57 \mathrm{E}+08$ \\
\hline ND-147 & 7 & $6.52 \mathrm{E}+07$ & $7.26 \mathrm{E}+07$ \\
\hline PR-143 & 7 & $1.46 \mathrm{E}+08$ & $1.62 E+08$ \\
\hline$Y-90$ & 7 & $5.62 E+06$ & $6.26 \mathrm{E}+06$ \\
\hline Y-91 & 7 & $1.18 \mathrm{E}+08$ & $1.32 E+08$ \\
\hline$Y-92$ & 7 & $1.30 \mathrm{E}+08$ & $1.45 E+08$ \\
\hline Y-93 & 7 & $1.47 \mathrm{E}+08$ & $1.64 E+08$ \\
\hline ZR-95 & 7 & $1.49 E+08$ & $1.66 E+08$ \\
\hline ZR-97 & 7 & $1.56 \mathrm{E}+08$ & $1.73 E+08$ \\
\hline CE-141 & 8 & $1.53 E+08$ & $1.70 E+08$ \\
\hline CE-143 & 8 & $1.48 \mathrm{E}+08$ & $1.65 E+08$ \\
\hline CE-144 & 8 & $9.20 \mathrm{E}+07$ & $1.03 E+08$ \\
\hline NP-239 & 8 & $1.75 \mathrm{E}+09$ & $1.95 \mathrm{E}+09$ \\
\hline PU-238 & 8 & $9.90 \mathrm{E}+04$ & $1.10 E+05$ \\
\hline PU-239 & 8 & $2.23 E+04$ & $2.49 E+04$ \\
\hline PU-240 & 8 & $2.82 \mathrm{E}+04$ & $3.14 \mathrm{E}+04$ \\
\hline PU-241 & 8 & $4.74 E+06$ & $5.28 E+06$ \\
\hline BA-139 & 9 & $1.70 E+08$ & $1.89 E+08$ \\
\hline BA-140 & 9 & $1.68 E+08$ & $1.87 E+08$ \\
\hline
\end{tabular}

*1.114 is factor used for adjustment of the MACCS default PWR core inventory. 
Table 4.1.1 Mean Early Fatalities Estimated by MACCS Within One Mile

Beyond the Exclusion Zone Boundary

Emergency Response: No Evacuation

Exclusion Zone: 1 (0.25 mile, except 6 which is 0.4 mile)

Reactors all scaled to 3800 MWth

80\% Weather with 3940 ft. Mixing Height

\begin{tabular}{|c|c|c|c|c|c|}
\hline \multirow[t]{2}{*}{ Source Term } & \multicolumn{5}{|c|}{ Population Case } \\
\hline & 1 & 2 & 5 & 6 & 7 \\
\hline RGG1 & 39 & 8 & 78 & 72 & 160 \\
\hline RGG2 & 0.2 & $<0.1$ & 0.3 & 0.1 & 0.7 \\
\hline RGG3 & 0.5 & $<0.1$ & 0.9 & 0.4 & 2 \\
\hline RGG4 & 0.3 & $<0.1$ & 0.7 & 0.3 & 1 \\
\hline RGG5 & 0 & 0 & 0 & 0 & 0 \\
\hline RLAS1 & 0.3 & $<0.1$ & 0.5 & 0.1 & 1 \\
\hline RLAS2 & 0.1 & $<0.1$ & 0.3 & 0.1 & 0.6 \\
\hline RLAS3 & 0.2 & $<0.1$ & 0.3 & 0.1 & 0.6 \\
\hline RLAS4 & 5 & 1 & 9 & 5 & 18 \\
\hline RLAS5 & 11 & 2 & 23 & 19 & 45 \\
\hline RLAS6 & 1 & 0.2 & 2 & 2 & 4 \\
\hline RLAS7 & $<0.1$ & $<0.1$ & $<0.1$ & $<0.1$ & $<0.1$ \\
\hline RPB1 & 22 & 4 & 43 & 39 & 86 \\
\hline RPB2 & 14 & 3 & 27 & 22 & 54 \\
\hline RPB3 & 5 & 1 & 10 & 6 & 20 \\
\hline RPB4 & $<0.1$ & $<0.1$ & $\because 0.1$ & 0 & $<0.1$ \\
\hline RPB5 & 0 & 0 & 0 & 0 & 0 \\
\hline RPB6 & 79 & 16 & 160 & 150 & 310 \\
\hline RSEQ1 & 120 & 25 & 240 & 250 & 480 \\
\hline RSEQ2 & 60 & 12 & 120 & 110 & 240 \\
\hline RSEQ3 & $<0.1$ & $<0.1$ & $<0.1$ & $<0.1$ & 0.1 \\
\hline RSEQ4 & 0 & 0 & 0 & 0 & 0 \\
\hline
\end{tabular}


Table 4.1.1 (Continued)

\begin{tabular}{||l|c|c|c|c|c||}
\hline \multirow{2}{*}{ Source Term } & \multicolumn{5}{|c|}{ Population Case } \\
\cline { 2 - 6 } & 1 & 2 & 5 & 6 & 7 \\
\hline RSEQ5 & 58 & 12 & 120 & 110 & 230 \\
\hline RSUR1 & 71 & 15 & 140 & 130 & 280 \\
\hline RSUR2 & 22 & 5 & 44 & 36 & 87 \\
\hline RSUR3 & 0 & 0 & 0 & 0 & 0 \\
\hline RSUR4 & 58 & 12 & 110 & 110 & 230 \\
\hline RZ1 & 50 & 10 & 98 & 95 & 200 \\
\hline RZ2 & 0.2 & $<0.1$ & 0.3 & $<0.1$ & 0.6 \\
\hline RZ3 & 0 & 0 & 0 & 0 & 0 \\
\hline RZ4 & 51 & 10 & 100 & 90 & 200 \\
\hline
\end{tabular}


Table 4.1.2 Changes to the Candidate Source Terms to Potentially Lead to One Early Fatality in the Mean as Estimated by MACCS

Emergency Response: No Evacuation

Population Distribution: Uniform 1000 People/Sq. Mi.

Exclusion Zone: 3 (0.4 mile)

Reactors all Scaled to 3800 MWth (Except RLAS6 after Adjustment)

80\% Weather with 3940 ft. Mixing Height

\begin{tabular}{|c|c|c||}
\hline Original Source Term & Original Release Time (hr) & $\begin{array}{c}\text { Modified Release Time } \\
\text { (hr) }\end{array}$ \\
\hline RGG3 & 14 & 13.4 \\
\hline RLAS3 & 16.9 & 4.2 \\
\hline RZ2 & 12 & 6.6 \\
\hline Source Term & Original Power (MWth) & Modified Power (MWth) \\
\hline RLAS6 & 3800 & 1979 \\
\hline
\end{tabular}

Table 4.1.3 Number of Early Fatalities (after Modification) as Estimated by MACCS

\begin{tabular}{|c|c|}
\hline Modified Source Term & 0.4-1.4 Miles \\
\hline MRGG3 & 0.97 \\
\hline MRLAS3 & 0.94 \\
\hline MRLAS6 & 0.84 \\
\hline MRZ2 & 0.99 \\
\hline
\end{tabular}


Table 4.1.4 Inventory Released Compared to the Shutdown Inventory for the Three Modified BWR Source Terms (No Evacuation Case)

\begin{tabular}{|c|c|c|c|c|c|}
\hline Isotope & Group & $\begin{array}{l}\text { Shutdown } \\
\text { (Ci) }\end{array}$ & $\begin{array}{l}\text { MGG3 } \\
\text { (Ci) }\end{array}$ & $\begin{array}{c}\text { MLAS3 } \\
\text { (Ci) }\end{array}$ & $\begin{array}{c}\text { MLAS6 } \\
\text { (Ci) }\end{array}$ \\
\hline CO-58 & 6 & $5.8 \mathrm{E}+5$ & $1.9 \mathrm{E}+2$ & $1.7 \mathrm{E}+3$ & $8.6 E+2$ \\
\hline CO-60 & 6 & $7.0 \mathrm{E}+5$ & $2.3 E+2$ & $2.1 E+3$ & $1.0 \mathrm{E}+3$ \\
\hline KR-85 & 1 & $9.5 E+5$ & $9.5 E+5$ & $9.5 \mathrm{E}+5$ & $6.7 E+5$ \\
\hline KR-85M & 1 & $3.5 \mathrm{E}+7$ & $7.7 E+6$ & $2.9 E+7$ & $7.6 \mathrm{E}+6$ \\
\hline KR-87 & 1 & $6.3 E+7$ & $3.1 E+5$ & $3.3 E+7$ & $7.2 \mathrm{E}+5$ \\
\hline KR-88 & 1 & $8.5 E+7$ & $7.6 \mathrm{E}+6$ & $6.4 \mathrm{E}+7$ & $9.3 E+6$ \\
\hline RB-86 & 3 & $5.3 E+4$ & $5.8 \mathrm{E}+2$ & $3.6 \mathrm{E}+3$ & $3.9 E+3$ \\
\hline SR-89 & 5 & $1.1 E+8$ & $4.1 E+5$ & $2.3 E+6$ & $3.9 E+6$ \\
\hline SR-90 & 5 & $7.5 \mathrm{E}+6$ & $2.9 \mathrm{E}+4$ & $1.6 \mathrm{E}+5$ & $2.7 E+5$ \\
\hline SR-91 & 5 & $1.4 \mathrm{E}+8$ & $2.6 \mathrm{E}+5$ & $2.7 E+6$ & $2.9 E+6$ \\
\hline SR-92 & 5 & $1.4 \mathrm{E}+8$ & $4.6 \mathrm{E}+4$ & $2.2 E+6$ & $7.7 \mathrm{E}+5$ \\
\hline$Y-90$ & 7 & $8.0 \mathrm{E}+6$ & $4.5 E+3$ & $2.0 \mathrm{E}+4$ & $4.3 E+4$ \\
\hline Y-91 & 7 & $1.3 \mathrm{E}+8$ & $3.0 \mathrm{E}+4$ & $3.0 \mathrm{E}+5$ & $4.0 \mathrm{E}+5$ \\
\hline Y-92 & 7 & $1.4 \mathrm{E}+8$ & $1.2 \mathrm{E}+5$ & $8.7 E+5$ & $1.5 \mathrm{E}+6$ \\
\hline Y-93 & 7 & $1.6 \mathrm{E}+8$ & $1.8 \mathrm{E}+4$ & $3.4 \mathrm{E}+5$ & $2.9 \mathrm{E}+5$ \\
\hline ZR-95 & 7 & 1.7E +8 & $3.7 \mathrm{E}+4$ & $3.9 E+5$ & $5.0 \mathrm{E}+5$ \\
\hline ZR-97 & 7 & $1.7 \mathrm{E}+8$ & $2.6 \mathrm{E}+4$ & $3.8 E+5$ & $3.8 \mathrm{E}+5$ \\
\hline NB-95 & 7 & $1.6 \mathrm{E}+8$ & $3.5 \mathrm{E}+4$ & $3.7 \mathrm{E}+5$ & $4.8 \mathrm{E}+5$ \\
\hline MO-99 & 6 & $1.8 \mathrm{E}+8$ & $5.5 E+4$ & $5.5 \mathrm{E}+5$ & $2.5 E+5$ \\
\hline TC-99M & 6 & $1.6 \mathrm{E}+8$ & $5.6 \mathrm{E}+4$ & $4.9 E+5$ & $2.5 E+5$ \\
\hline RU-103 & 6 & $1.4 \mathrm{E}+8$ & $4.6 \mathrm{E}+4$ & $4.2 E+5$ & 2.1E+5 \\
\hline RU-105 & 6 & $9.3 E+7$ & $6.7 \mathrm{E}+3$ & $2.3 E+5$ & $4.3 E+4$ \\
\hline RU-106 & 6 & $3.8 \mathrm{E}+7$ & $1.3 E+4$ & $1.1 E+5$ & $5.7 E+4$ \\
\hline RH-105 & 6 & $7.0 \mathrm{E}+7$ & $2.2 \mathrm{E}+4$ & $2.1 E+5$ & $1.0 E+5$ \\
\hline SB-127 & 4 & $8.8 E+6$ & $6.4 E+4$ & $3.9 E+5$ & $5.0 E+5$ \\
\hline SB-129 & 4 & $3.1 E+7$ & $5.1 E+4$ & $1.1 E+6$ & $5.5 E+5$ \\
\hline TE-127 & 4 & $8.6 E+6$ & $6.6 E+4$ & $3.9 \mathrm{E}+5$ & $5.1 E+5$ \\
\hline TE-127M & 4 & $1.2 \mathrm{E}+6$ & $8.9 \mathrm{E}+3$ & $5.2 \mathrm{E}+4$ & $6.8 \mathrm{E}+4$ \\
\hline TE-129 & 4 & $2.9 \mathrm{E}+7$ & $6.9 E+4$ & $1.3 \mathrm{E}+6$ & $7.4 \mathrm{E}+5$ \\
\hline
\end{tabular}


Table 4.1.4 (Continued)

\begin{tabular}{|c|c|c|c|c|c|}
\hline Isotope & Group & $\begin{array}{l}\text { Shutdown } \\
\text { (Ci) }\end{array}$ & $\begin{array}{c}\text { MGG3 } \\
\text { (Ci) }\end{array}$ & $\begin{array}{c}\text { MLAS3 } \\
\text { (Ci) }\end{array}$ & $\begin{array}{c}\text { MLAS6 } \\
\text { (Ci) }\end{array}$ \\
\hline TE-129M & 4 & $7.6 \mathrm{E}+6$ & $5.8 E+4$ & $3.4 E+5$ & $4.5 E+5$ \\
\hline TE-131M & 4 & $1.5 \mathrm{E}+7$ & $9.0 \mathrm{E}+4$ & $6.3 E+5$ & $7.2 E+5$ \\
\hline TE-132 & 4 & $1.4 \mathrm{E}+8$ & $1.0 \mathrm{E}+6$ & $6.3 E+6$ & $7.9 E+6$ \\
\hline I-131 & 2 & $9.8 \mathrm{E}+7$ & $1.4 E+7$ & $1.1 E+7$ & $6.6 E+6$ \\
\hline I-132 & 2 & $1.4 \mathrm{E}+8$ & $\therefore 1 E+6$ & $1.3 E+7$ & $8.2 E+6$ \\
\hline I-133 & 2 & $2.1 E+8$ & $2.2 \mathrm{E}+7$ & $2.2 \mathrm{E}+7$ & $1.1 E+7$ \\
\hline I-134 & 2 & $2.3 E+8$ & $1.5 \mathrm{E}+4$ & $9.0 \mathrm{E}+6$ & $4.1 E+4$ \\
\hline I-135 & 2 & $1.9 \mathrm{E}+8$ & $1.0 \mathrm{E}+7$ & $1.9 E+7$ & $6.1 E+6$ \\
\hline XE-133 & 1 & $2.1 E+8$ & $2.0 \mathrm{E}+8$ & 2.1E+8 & $1.4 \mathrm{E}+8$ \\
\hline XE-135 & 1 & $4.9 E+7$ & $3.2 \mathrm{E}+7$ & 4.7E +7 & $2.3 \mathrm{E}+7$ \\
\hline CS-134 & 3 & $1.6 \mathrm{E}+7$ & $1.8 \mathrm{E}+5$ & $1.1 E+6$ & $1.2 \mathrm{E}+6$ \\
\hline CS-136 & 3 & $4.3 E+6$ & $4.6 \mathrm{E}+4$ & $2.9 E+5$ & $3.1 E+5$ \\
\hline CS-137 & 3 & $9.6 E+6$ & $1.1 \mathrm{E}+5$ & $6.4 E+5$ & $7.1 \mathrm{E}+5$ \\
\hline BA-139 & 9 & $1.9 \mathrm{E}+8$ & $4.8 \mathrm{E}+3$ & $2.2 E+6$ & $1.4 \mathrm{E}+5$ \\
\hline BA-140 & 9 & $1.9 \mathrm{E}+8$ & $6.0 \mathrm{E}+5$ & 4.2E +6 & $5.9 E+6$ \\
\hline LA-140 & 7 & $1.9 \mathrm{E}+8$ & $1.3 \mathrm{E}+5$ & $5.3 E+5$ & $1.2 \mathrm{E}+6$ \\
\hline LA-141 & 7 & $1.8 \mathrm{E}+8$ & $7.0 \mathrm{E}+3$ & $3.2 E+5$ & $1.4 \mathrm{E}+5$ \\
\hline LA-142 & 7 & $1.7 E+8$ & $5.4 \mathrm{E}+2$ & $2.2 \mathrm{E}+5$ & $1.9 E+4$ \\
\hline CE-141 & 8 & $1.7 E+8$ & $5.6 \mathrm{E}+4$ & $3.9 E+5$ & $6.5 E+5$ \\
\hline CE-143 & 8 & $1.7 \mathrm{E}+8$ & $4.5 \mathrm{E}+4$ & $3.7 \mathrm{E}+5$ & $5.4 \mathrm{E}+5$ \\
\hline CE-144 & 8 & $1.1 \mathrm{E}+8$ & $3.6 \mathrm{E}+4$ & $2.5 \mathrm{E}+5$ & $4.2 E+5$ \\
\hline PR-143 & 7 & $1.6 \mathrm{E}+8$ & $3.6 \mathrm{E}+4$ & $3.7 E+5$ & $4.8 E+5$ \\
\hline ND-147 & 7 & $7.2 \mathrm{E}+7$ & $1.6 \mathrm{E}+4$ & $1.7 E+5$ & $2.1 E+5$ \\
\hline NP-239 & 8 & $2.2 \mathrm{E}+9$ & $6.3 E+5$ & $4.9 E+6$ & $7.6 \mathrm{E}+6$ \\
\hline PU-238 & 8 & $1.5 \mathrm{E}+5$ & $5.0 \mathrm{E}+1$ & $3.5 E+2$ & $5.8 \mathrm{E}+2$ \\
\hline PU-239 & 8 & $3.8 E+4$ & $1.3 \mathrm{E}+1$ & $8.8 E+1$ & $1.5 \mathrm{E}+2$ \\
\hline PU-240 & 8 & $4.8 E+4$ & $1.6 E+1$ & $1.1 E+2$ & $1.8 \mathrm{E}+2$ \\
\hline PU-241 & 8 & $8.2 E+6$ & $2.7 E+3$ & $1.9 E+4$ & $3.2 E+4$ \\
\hline AM-241 & 7 & $8.3 E+3$ & $1.8 \mathrm{E}+0$ & $1.9 E+1$ & $2.5 \mathrm{E}+1$ \\
\hline CM-242 & 7 & $2.2 \mathrm{E}+6$ & $4.8 E+2$ & $5.1 E+3$ & $6.5 \mathrm{E}+3$ \\
\hline CM-244 & 7 & $1.2 \mathrm{E}+5$ & $2.6 \mathrm{E}+1$ & $2.7 \mathrm{E}+2$ & $3.5 \mathrm{E}+2$ \\
\hline
\end{tabular}


Table 4.1.5 Fraction of the Shutdown Inventory Released for the Three Modified BWR Source Terms (No Evacuation Case)

\begin{tabular}{|c|c|c|c|}
\hline Isotope & MGG3 & MLAS3 & MLAS6 \\
\hline $\mathrm{CO}-58$ & $3.3 \mathrm{E}-04$ & $3.0 \mathrm{E}-03$ & $1.5 \mathrm{E}-03$ \\
\hline$c 0-60$ & 3.3E-04 & $3.0 \mathrm{E}-03$ & 1.5E-03 \\
\hline KR-85 & $1.0 \mathrm{E}+00$ & $1.0 \mathrm{E}+00$ & 7.1E-01 \\
\hline KR-85M & $2.2 \mathrm{E}-01$ & 8.3E-01 & 2.2E-01 \\
\hline KR-87 & $4.9 E-03$ & $5.3 E-01$ & $1.1 \mathrm{E}-02$ \\
\hline KR-88 & $9.0 \mathrm{E}-02$ & $7.5 \mathrm{E}-01$ & $1.1 \mathrm{E}-01$ \\
\hline RB-86 & $1.1 \mathrm{E}-02$ & $6.7 \mathrm{E}-02$ & 7.3E-02 \\
\hline SR-89 & $3.9 \mathrm{E}-03$ & 2.2E-02 & 3.7E-02 \\
\hline SR-90 & $3.9 \mathrm{E}-03$ & $2.2 \mathrm{E}-02$ & 3.7E-02 \\
\hline SR-91 & $1.9 \mathrm{E}-03$ & $2.0 \mathrm{E}-02$ & 2. $1 \mathrm{E}-02$ \\
\hline SR-92 & 3.2E-04 & $1.6 \mathrm{E}-02$ & $5.4 \mathrm{E}-03$ \\
\hline$Y-90$ & $5.6 \mathrm{E}-04$ & $2.6 \mathrm{E}-03$ & $5.4 \mathrm{E}-03$ \\
\hline$Y-91$ & 2.3E-04 & $2.3 E-03$ & $3.1 \mathrm{E}-03$ \\
\hline Y-92 & 8.7E-04 & $6.0 \mathrm{E}-03$ & $1.1 \mathrm{E}-02$ \\
\hline Y-93 & 1.1E-04 & 2.1E-03 & $1.8 \mathrm{E}-03$ \\
\hline ZR-95 & 2.2E-04 & 2.3E-03 & $3.0 \mathrm{E}-03$ \\
\hline ZR-97 & 1.5E-04 & 2.2E-03 & $2.2 \mathrm{E}-03$ \\
\hline NB-95 & 2.2E-04 & 2.3E-03 & $3.0 \mathrm{E}-03$ \\
\hline MO-99 & 3.0E-04 & 3.0E-03 & $1.4 \mathrm{E}-03$ \\
\hline TC-99M & 3.5E-04 & $3.1 E-03$ & $1.6 \mathrm{E}-03$ \\
\hline RU-103 & 3.3E-04 & $3.0 \mathrm{E}-03$ & $1.5 E-03$ \\
\hline RU-105 & 7.2E-05 & $2.5 \mathrm{E}-03$ & 4.6E-04 \\
\hline RU-106 & 3.3E-04 & $3.0 \mathrm{E}-03$ & $1.5 \mathrm{E}-03$ \\
\hline RH-105 & 3.1E-04 & $3.0 \mathrm{E}-03$ & $1.4 \mathrm{E}-03$ \\
\hline SB-127 & 7.2E-03 & 4.4E-02 & $5.6 \mathrm{E}-02$ \\
\hline SB-129 & $1.6 \mathrm{E}-03$ & $3.6 \mathrm{E}-02$ & $1.8 \mathrm{E}-02$ \\
\hline TE-127 & $7.8 \mathrm{E}-03$ & $4.5 \mathrm{E}-02$ & $5.9 \mathrm{E}-02$ \\
\hline TE-127M & $7.8 \mathrm{E}-03$ & 4.5E-02 & $5.9 \mathrm{E}-02$ \\
\hline TE-129 & $2.4 \mathrm{E}-03$ & 4.4E-02 & $2.6 \mathrm{E}-02$ \\
\hline TE-129M & 7.7E-03 & 4.5E-02 & $5.9 \mathrm{E}-02$ \\
\hline
\end{tabular}


Table 4.1.5 (Continued)

\begin{tabular}{|c|c|c|c|}
\hline Isotope & MGG3 & MLAS3 & MLAS6 \\
\hline TE-131M & $6.2 \mathrm{E}-03$ & $4.4 \mathrm{E}-02$ & $5.0 \mathrm{E}-02$ \\
\hline TE-132 & 7.2E-03 & 4.4E-02 & $5.6 \mathrm{E}-02$ \\
\hline I-131 & $1.4 \mathrm{E}-01$ & $1.1 \mathrm{E}-01$ & 6.7E-02 \\
\hline I-132 & $1.4 \mathrm{E}-02$ & 9.1E-02 & $5.7 \mathrm{E}-02$ \\
\hline I-133 & $1.0 \mathrm{E}-01$ & $1.1 \mathrm{E}-01$ & $5.4 \mathrm{E}-02$ \\
\hline I-134 & $6.6 \mathrm{E}-05$ & 4.0E-02 & $1.8 \mathrm{E}-04$ \\
\hline I-135 & $5.2 \mathrm{E}-02$ & $9.9 \mathrm{E}-02$ & $3.1 \mathrm{E}-02$ \\
\hline XE-133 & $9.5 \mathrm{E}-01$ & $1.0 \mathrm{E}+00$ & $6.8 \mathrm{E}-01$ \\
\hline XE-135 & $6.6 E-01$ & $9.6 \mathrm{E}-01$ & $4.8 \mathrm{E}-01$ \\
\hline CS-134 & $1.1 \mathrm{E}-02$ & 6.7E-02 & 7.3E-02 \\
\hline CS-136 & $1.1 \mathrm{E}-02$ & $6.7 \mathrm{E}-02$ & 7.2E-02 \\
\hline CS-137 & $1.1 \mathrm{E}-02$ & $6.7 \mathrm{E}-02$ & 7.3E-02 \\
\hline BA-139 & $2.5 \mathrm{E}-05$ & $1.2 \mathrm{E}-02$ & $.7 .5 \mathrm{E}-04$ \\
\hline BA-140 & $3.2 E-03$ & $2.3 \mathrm{E}-02$ & $3.2 \mathrm{E}-02$ \\
\hline LA-140 & $6.8 \mathrm{E}-04$ & $2.8 \mathrm{E}-03$ & $6.4 \mathrm{E}-03$ \\
\hline LA-141 & 4.0E-05 & $1.8 \mathrm{E}-03$ & $7.9 \mathrm{E}-04$ \\
\hline LA-142 & $3.2 \mathrm{E}-06$ & $1.3 \mathrm{E}-03$ & 1.1E-04 \\
\hline CE-141 & 3.3E-04 & $2.3 \mathrm{E}-03$ & $3.8 \mathrm{E}-03$ \\
\hline CE-143 & $2.7 \mathrm{E}-04$ & 2.2E-03 & $3.3 E-03$ \\
\hline CE-144 & $3.3 \mathrm{E}-04$ & 2.3E-03 & $3.8 \mathrm{E}-03$ \\
\hline PR-143 & 2.2E-04 & 2.3E-03 & $3.0 \mathrm{E}-03$ \\
\hline ND-147 & $2.1 E-04$ & 2.3E-03 & $2.9 \mathrm{E}-03$ \\
\hline NP-239 & $2.9 E-04$ & 2.3E-03 & $3.5 \mathrm{E}-03$ \\
\hline PU-238 & $3.3 E-04$ & 2.3E-03 & $3.8 \mathrm{E}-03$ \\
\hline PU-239 & 3.3E-04 & 2.3E-03 & $3.8 \mathrm{E}-03$ \\
\hline PU-240 & $3.3 E-04$ & $2.3 E-03$ & 3.8E-03 \\
\hline PU-241 & $3.3 E-04$ & 2.3E-03 & $3.8 \mathrm{E}-03$ \\
\hline AM-241 & 2.2E-04 & 2.3E-03 & $3.0 \mathrm{E}-03$ \\
\hline CM-242 & $2.2 \mathrm{E}-04$ & 2.3E-03 & $3.0 \mathrm{E}-03$ \\
\hline CM-244 & 2.2E-04 & $2.3 E-03$ & $3.0 \mathrm{E}-03$ \\
\hline
\end{tabular}


4 Results

Table 4.1.6 Inventory Released Compared to the Shutdown Inventory for the Three Modified BWR Source Terms Grouped by Isotope Grouping (No Evacuation Case)

\begin{tabular}{|c|c|c|c|c||}
\hline Group & Shutdown & MGG3 & MLAS3 & MLAS6 \\
\hline 1 & $4.4 \mathrm{E}+08$ & $2.5 \mathrm{E}+08$ & $3.8 \mathrm{E}+08$ & $1.8 \mathrm{E}+08$ \\
\hline 2 & $8.7 \mathrm{E}+08$ & $4.8 \mathrm{E}+07$ & $7.5 \mathrm{E}+07$ & $3.2 \mathrm{E}+07$ \\
\hline 3 & $3.0 \mathrm{E}+07$ & $3.3 \mathrm{E}+05$ & $2.0 \mathrm{E}+06$ & $2.2 \mathrm{E}+06$ \\
\hline 4 & $2.4 \mathrm{E}+08$ & $1.4 \mathrm{E}+06$ & $1.0 \mathrm{E}+07$ & $1.1 \mathrm{E}+07$ \\
\hline 5 & $3.9 \mathrm{E}+08$ & $7.5 \mathrm{E}+05$ & $7.4 \mathrm{E}+06$ & $7.8 \mathrm{E}+06$ \\
\hline 6 & $6.9 \mathrm{E}+08$ & $2.0 \mathrm{E}+05$ & $2.0 \mathrm{E}+06$ & $9.2 \mathrm{E}+05$ \\
\hline 7 & $1.7 \mathrm{E}+09$ & $4.6 \mathrm{E}+05$ & $4.3 \mathrm{E}+06$ & $5.7 \mathrm{E}+06$ \\
\hline 8 & $2.6 \mathrm{E}+09$ & $7.7 \mathrm{E}+05$ & $5.9 \mathrm{E}+06$ & $9.2 \mathrm{E}+06$ \\
\hline 9 & $3.8 \mathrm{E}+08$ & $6.1 \mathrm{E}+05$ & $6.4 \mathrm{E}+06$ & $6.1 \mathrm{E}+06$ \\
\hline
\end{tabular}

Table 4.1.7 Fraction of Shutdown Inventory Released for the Three Modified BWR Source Terms Grouped by Isotope Grouping (No Evacuation Case)

\begin{tabular}{|c|c|c|c||}
\hline Group & MGG3 & MLAS3 & MLAS6 \\
\hline 1 & $5.9 \mathrm{E}-01$ & $9.2 \mathrm{E}-01$ & $4.4 \mathrm{E}-01$ \\
\hline 2 & $5.8 \mathrm{E}-02$ & $9.2 \mathrm{E}-02$ & $3.9 \mathrm{E}-02$ \\
\hline 3 & $1.2 \mathrm{E}-02$ & $7.1 \mathrm{E}-02$ & $7.8 \mathrm{E}-02$ \\
\hline 4 & $6.2 \mathrm{E}-03$ & $4.6 \mathrm{E}-02$ & $5.0 \mathrm{E}-02$ \\
\hline 5 & $2.0 \mathrm{E}-03$ & $2.0 \mathrm{E}-02$ & $2.1 \mathrm{E}-02$ \\
\hline 6 & $3.1 \mathrm{E}-04$ & $3.1 \mathrm{E}-03$ & $1.4 \mathrm{E}-03$ \\
\hline 7 & $2.9 \mathrm{E}-04$ & $2.6 \mathrm{E}-03$ & $3.5 \mathrm{E}-03$ \\
\hline 8 & $3.1 \mathrm{E}-04$ & $2.4 \mathrm{E}-03$ & $3.7 \mathrm{E}-03$ \\
\hline 9 & $1.7 \mathrm{E}-03$ & $1.8 \mathrm{E}-02$ & $1.7 \mathrm{E}-02$ \\
\hline
\end{tabular}


Table 4.1.8 Inventory Released Compared to the Shutdown Core Inventory for the Modified PWR Source Term (No Evacuation Case)

\begin{tabular}{|c|c|c|c|c|}
\hline \multirow[t]{2}{*}{ Isotope } & \multirow[t]{2}{*}{ Group } & \multirow{2}{*}{$\begin{array}{l}\text { Core Inventory } \\
\text { (Ci) }\end{array}$} & \multicolumn{2}{|c|}{ MZ2 } \\
\hline & & & (Ci) & (Fraction) \\
\hline CO-58 & 6 & $9.7 \mathrm{E}+05$ & $1.9 \mathrm{E}-02$ & $2.0 \mathrm{E}-08$ \\
\hline CO-60 & 6 & $7.4 E+05$ & $1.5 \mathrm{E}-02$ & $2.0 \mathrm{E}-08$ \\
\hline KR-85 & 1 & $7.5 E+05$ & $7.5 \mathrm{E}+05$ & $1.0 \mathrm{E}+00$ \\
\hline KR-85M & 1 & $3.5 \mathrm{E}+07$ & $1.4 E+07$ & $3.9 \mathrm{E}-01$ \\
\hline KR-87 & 1 & $6.4 E+07$ & $2.3 E+06$ & $3.5 \mathrm{E}-02$ \\
\hline KR-88 & 1 & $8.6 E+07$ & $1.9 E+07$ & 2.2E-01 \\
\hline RB-86 & 3 & 5.7E +04 & $3.4 \mathrm{E}-01$ & $5.9 \mathrm{E}-06$ \\
\hline SR-89 & 5 & $1.1 E+08$ & $1.1 E+02$ & $1.0 \mathrm{E}-06$ \\
\hline SR-90 & 5 & $5.8 \mathrm{E}+06$ & $5.8 \mathrm{E}+\infty 0$ & $1.0 \mathrm{E}-06$ \\
\hline SR-91 & 5 & $1.4 \mathrm{E}+08$ & $8.9 E+01$ & $6.4 \mathrm{E}-07$ \\
\hline SR-92 & 5 & $1.4 \mathrm{E}+08$ & $3.0 \mathrm{E}+01$ & $2.1 \mathrm{E}-07$ \\
\hline$Y-90$ & 7 & $6.3 \mathrm{E}+06$ & $9.6 \mathrm{E}-01$ & $1.5 \mathrm{E}-07$ \\
\hline$Y-91$ & 7 & $1.3 E+08$ & $1.3 \mathrm{E}+01$ & $1.0 \mathrm{E}-07$ \\
\hline$Y-92$ & 7 & $1.5 \mathrm{E}+08$ & $4.8 \mathrm{E}+01$ & 3.3E-07 \\
\hline$Y-93$ & 7 & $1.6 \mathrm{E}+08$ & $1.1 \mathrm{E}+01$ & $6.6 \mathrm{E}-08$ \\
\hline ZR-95 & 7 & $1.7 \mathrm{E}+08$ & $1.7 \mathrm{E}+01$ & 1.0E-07 \\
\hline ZR-97 & 7 & 1.7E +08 & $1.3 \mathrm{E}+01$ & $7.8 \mathrm{E}-08$ \\
\hline NB-95 & 7 & $1.6 \mathrm{E}+08$ & $1.6 \mathrm{E}+01$ & $1.0 E-07$ \\
\hline MO-99 & 6 & $1.8 \mathrm{E}+08$ & $3.4 \mathrm{E}+00$ & $1.9 \mathrm{E}-08$ \\
\hline TC-99M & 6 & $1.6 \mathrm{E}+08$ & $3.4 \mathrm{E}+00$ & 2.1E-08 \\
\hline RU-103 & 6 & $1.4 \mathrm{E}+08$ & $2.7 \mathrm{E}+00$ & $2.0 \mathrm{E}-08$ \\
\hline RU-105 & 6 & $8.9 E+07$ & $6.8 \mathrm{E}-01$ & 7.7E-09 \\
\hline RU-106 & 6 & $3.1 E+07$ & $6.2 \mathrm{E}-01$ & $2.0 \mathrm{E}-08$ \\
\hline RH-105 & 6 & $6.2 E+07$ & $1.2 \mathrm{E}+\infty 0$ & $2.0 \mathrm{E}-08$ \\
\hline SB-127 & 4 & $8.4 E+06$ & $5.6 \mathrm{E}+01$ & $6.7 \mathrm{E}-06$ \\
\hline SB-129 & 4 & $3.0 \mathrm{E}+07$ & $7.8 \mathrm{E}+01$ & $2.6 \mathrm{E}-06$ \\
\hline TE-127 & 4 & $8.1 E+06$ & $5.7 E+01$ & $7.0 \mathrm{E}-06$ \\
\hline TE-127M & 4 & $1.1 \mathrm{E}+06$ & $7.5 \mathrm{E}+00$ & $7.0 E-06$ \\
\hline TE-129 & 4 & $2.8 E+07$ & $1.0 E+02$ & $3.8 \mathrm{E}-06$ \\
\hline TE-129M & 4 & $7.4 E+06$ & $5.1 E+01$ & $7.0 E-06$ \\
\hline
\end{tabular}


Table 4.1.8 (Continued)

\begin{tabular}{|c|c|c|c|c|}
\hline \multirow[t]{2}{*}{ Isotope } & \multirow[t]{2}{*}{ Group } & \multirow{2}{*}{$\begin{array}{l}\text { Core Inventory } \\
\text { (Ci) }\end{array}$} & \multicolumn{2}{|c|}{ MZ2 } \\
\hline & & & (Ci) & (Fraction) \\
\hline TE-131M & 4 & $1.4 E+07$ & $8.6 E+01$ & 6.1E-06 \\
\hline TE-132 & 4 & $1.4 E+08$ & $9.3 E+02$ & $6.6 \mathrm{E}-06$ \\
\hline $\mid-131$ & 2 & $9.7 E+07$ & $2.8 \mathrm{E}+06$ & 2.9E-02 \\
\hline 1-132 & 2 & $1.4 E+08$ & $6.7 E+05$ & 4.7E-03 \\
\hline $1-133$ & 2 & $2.0 E+08$ & $5.0 \mathrm{E}+06$ & $2.4 \mathrm{E}-02$ \\
\hline $1-134$ & 2 & $2.2 E+08$ & $5.4 \mathrm{E}+04$ & $2.4 \mathrm{E}-04$ \\
\hline 1-135 & 2 & $1.9 E+08$ & $3.0 \mathrm{E}+06$ & 1.6E-02 \\
\hline$X E-133$ & 1 & $2.0 E+08$ & $2.0 \mathrm{E}+08$ & $9.7 E-01$ \\
\hline XE-135 & 1 & $3.8 \mathrm{E}+07$ & $2.6 \mathrm{E}+07$ & $6.7 \mathrm{E}-01$ \\
\hline CS-134 & 3 & $1.3 E+07$ & $7.8 \mathrm{E}+01$ & $6.0 \mathrm{E}-06$ \\
\hline CS-136 & 3 & $4.0 \mathrm{E}+06$ & $2.3 \mathrm{E}+01$ & $5.9 E-06$ \\
\hline CS-137 & 3 & $7.3 \mathrm{E}+06$ & $4.4 \mathrm{E}+01$ & $6.0 \mathrm{E}-06$ \\
\hline BA-139 & 9 & $1.9 E+08$ & $8.9 E+\infty 0$ & 4.7E-08 \\
\hline BA-140 & 9 & $1.9 E+08$ & $1.8 \mathrm{E}+02$ & $9.9 E-07$ \\
\hline LA-140 & 7 & $1.9 E+08$ & $3.6 \mathrm{E}+01$ & $1.9 \mathrm{E}-07$ \\
\hline LA-141 & 7 & $1.8 E+08$ & $6.0 \mathrm{E}+00$ & $3.4 \mathrm{E}-08$ \\
\hline LA-142 & 7 & $1.7 \mathrm{E}+08$ & $1.2 E+00$ & $7.0 \mathrm{E}-09$ \\
\hline CE-141 & 8 & $1.7 \mathrm{E}+08$ & $1.7 \mathrm{E}+01$ & $1.0 \mathrm{E}-07$ \\
\hline CE-143 & 8 & $1.7 E+08$ & $1.5 E+01$ & 8.8E-08 \\
\hline CE-144 & 8 & $1.0 E+08$ & $1.0 \mathrm{E}+01$ & $1.0 \mathrm{E}-07$ \\
\hline PR-143 & 7 & $1.6 E+08$ & $1.6 E+01$ & 1.0E-07 \\
\hline ND-147 & 7 & $7.3 E+07$ & $7.1 \mathrm{E}+\infty$ & $9.8 E-08$ \\
\hline NP-239 & 8 & $1.9 \mathrm{E}+09$ & $1.8 \mathrm{E}+02$ & $9.3 E-08$ \\
\hline PU-238 & 8 & $1.1 E+05$ & $1.1 E-02$ & $1.0 \mathrm{E}-07$ \\
\hline PU-239 & 8 & $2.5 E+04$ & $2.5 E-03$ & $1.0 \mathrm{E}-07$ \\
\hline PU-240 & 8 & $3.1 \mathrm{E}+04$ & $3.1 \mathrm{E}-03$ & $1.0 \mathrm{E}-07$ \\
\hline PU-241 & 8 & $5.3 E+06$ & 5.3E-01 & $1.0 \mathrm{E}-07$ \\
\hline AM-241 & 7 & $3.5 E+03$ & 3.5E-04 & $1.0 \mathrm{E}-07$ \\
\hline CM-242 & 7 & $1.3 E+06$ & $1.3 \mathrm{E}-01$ & $1.0 \mathrm{E}-07$ \\
\hline CM-244 & 7 & $7.8 E+04$ & $7.8 \mathrm{E}-03$ & $1.0 \mathrm{E}-07$ \\
\hline
\end{tabular}


Table 4.1.9 Released Inventory and Fractional Release for the Modified PWR Source Term Grouped by Isotope Grouping (No Evacuation Case)

\begin{tabular}{|c|c|c|c|}
\hline \multirow{2}{*}{ Group } & Core Inventory & \multicolumn{2}{|c|}{ MZ2 } \\
\cline { 2 - 4 } & $(\mathrm{Ci})$ & $(\mathrm{Ci})$ & (Fraction) \\
\hline 1 & $4.3 \mathrm{E}+08$ & $2.6 \mathrm{E}+08$ & $6.0 \mathrm{E}-01$ \\
\hline 2 & $8.6 \mathrm{E}+08$ & $1.2 \mathrm{E}+07$ & $1.3 \mathrm{E}-02$ \\
\hline 3 & $2.4 \mathrm{E}+07$ & $1.5 \mathrm{E}+02$ & $6.0 \mathrm{E}-06$ \\
\hline 4 & $2.4 \mathrm{E}+08$ & $1.4 \mathrm{E}+03$ & $5.8 \mathrm{E}-06$ \\
\hline 5 & $4.0 \mathrm{E}+08$ & $2.3 \mathrm{E}+02$ & $5.9 \mathrm{E}-07$ \\
\hline 6 & $6.6 \mathrm{E}+08$ & $1.2 \mathrm{E}+01$ & $1.8 \mathrm{E}-08$ \\
\hline 7 & $1.7 \mathrm{E}+09$ & $1.9 \mathrm{E}+02$ & $1.1 \mathrm{E}-07$ \\
\hline 8 & $2.4 \mathrm{E}+09$ & $2.2 \mathrm{E}+02$ & $9.3 \mathrm{E}-08$ \\
\hline 9 & $3.8 \mathrm{E}+08$ & $1.9 \mathrm{E}+02$ & $5.1 \mathrm{E}-07$ \\
\hline
\end{tabular}




\subsection{Source Terms for the Mean Evacuation Case}

For the mean evacuation case, candidate source terms were identified and modified to have the potential to lead to one mean early fatality as estimated by MACCS. Initially, all simplified source terms and population cases were considered. The results are presented in Table 4.2.1, which shows the number of early fatalities estimated by MACCS within the first mile beyond the exclusion boundary.

Three source terms, RPBI, RPB6, and RSUR2, were selected for modification to result in an estimated one mean early fatality. As in Section 4.1, Population Case 6 (1000 people per square mile and 0.4 miles exclusion boundary) was the focus of the consequence calculations. For these source terms, the only change made was to adjust the core inventory (i.e., the reactor power level) until the desired result of approximately one mean early fatality was achieved, with the exception of the noble gases which were maintained at the $3800 \mathrm{MWth}$ level. The modifications necessary are shown in Table 4.2.2.

A key factor which affects the calculation of early fatalities is the time when evacuation occurs relative to the time at which the release begins. For a given emergency response time, very early containment failure, where the release starts before evacuation can begin, can lead to more early fatalities than late containment failures.

To facilitate discussion of the time of release parameter (time at which release occurs relative to the time at which warning is given) as a factor potentially influencing the definition of a large release sourco term, candidate source terms were qualitatively binned into early, mid, and late categories. For this purpose early releases were considered to have release times $\leq 4$ hours, mid release between 4 and 8 hours, and late releases greater than 8 hours. Table 4.2.2 includes the release time categories for each of the source terms selected for modification.

Table 4.2.3 displays the MACCS estimated number of early fatalities for each of the modified source terms. Given the release times of the source terms, the timing of the evacuation, and the assumption that 99.5 percent of the population participates in the evacuation, it was necessary to consider distances beyond one mile from the site boundary to arrive at the potential for one mean early fatality. The contributions to the early fatalities from the ovacuated population ( $99.5 \%$ of the total) and the non-evacuated population $(0.5 \%)$ are shown separately.

Out to one mile from the exclusion boundary, each of the modified source terms leads to an estimated 0.5 to 0.6 mean early fatalities. Based on a density of 1000 per square mile, the mean of the population weighted early fatalities one mile from the exclusion boundary is about $1.0 \mathrm{E}-4$ in this case.

Tables 4.2.4 and 4.2.5 show the released inventory for the modified BWR source terms MPB1 and MPB6 by individual isotope and isotope groups and as fractions of the shutdown inventories. Tables 4.2.6 and 4.2.7 display the corresponding quantities for the modified PWR source term MSUR2.

The magnitude of the releases required to have the potential to lead to one early fatality in the mean evacuation case is extremely large. It is interesting to consider what contributes to the MACCS estimated number of early fatalities. Table 4.2.8 shows the MACCS estimated number of early fatalities for the three modified source terms, and the contributions from the evacuating and the non-evacuating population. (As stated above, it was necessary in this case to consider distances beyond one mile to achieve the potential for one early fatality). The MACCS estimated total number of early fatalities is the sum of the number experienced by each emergency action (evacuees vs. nonevacuees) multiplied by the respective fraction of the population in each cohort. This table indicates that the evacuees for the three source terms examined do not receive enough radiation exposure to produce even a single 
estimated fatality within this group. Only for the population that does not evacuate does MACCS estimate early fatalities.

This observation is supported by the results provided in Table 4.2.9, the number of individuals receiving exposure exceeding the threshold doses to the three critical organs (red marrow, lungs, lower large intestine) evaluated in the MACCS early fatality health effects model. For the evacuees, there were no exposures exceeding the thresholds for early fatality. All of the exposure exceeding the thresholds was experienced by the population that did not evacuate.

Tables B.2.1-B.2.3 in Appendix B provide the centerline doses to red marrow, lungs, and lower large intestine (LLI) with distance and for the different pathways that make up the acute (seven day) exposure. Results are given for the RPB1 source term. Inspection of these tables indicate that the dose to the evacuees is very small compared to the dose to the non-evacuees. For non-evacuees, the major contributor to the red marrow dose is from the seven day groundshine, which is the major contributor to early fatalities. For lung and LLI, the major doses arise from inhalation, with the groundshine contribution being about $60 \%$ of the inhalation contribution. These three tables are used to derive the information in Tables B.2.4-B.2.6 which show the relative fractions of dose at each distance for each pathway. These fractions are displayed by protective action assumption; that is, the total population, the evacuated population, and the non-evacuated population. Since the dose to the total population is dominated by the dose to the non-evacuated population, it is not surprising that the relative fractions of dose to the total population follows the relative fractions of dose to the non-evacuated population.

Of the population that participates in the evacuation, the inhalation dose is the dominant contributor close in. For the lungs and LLI, the inhalation contribution is the overwbelming factor at all distances where non-zero results were obtained.

In summary, for the three modified source terms in the mean evacuation case, early fatalities were limited to the small fraction of the population that did not participate in the evacuation. For the accidents examined, the evacuation was effective. There was sufficient time after waming was issued to execute the evacuation. For the sequences PB1 and PB2, the evacuees nearest the reactor have already moved 9 miles at the time of release. In the SUR2 sequence, the evacuation out to 10 miles has been completed. The magnitude of these source terms, that is the fractional releases, is therefore very large. In fact, several isotopic releases exceed those that are estimated to have occurred during the Chernobyl accident. 
4 Results

Table 4.2.1 Estimated Number of Mean Early Fatalities 1 Mile Beyond Exclusion Boundary Emergency Response: Mean Evacuation

Exclusion Zone: 1 (0.25 mile, except Case 6 which is 0.4 mile)

Reactors All Scaled to 3800 MWth

80\% Weather with 3940 ft. Mixing Height

\begin{tabular}{|c|c|c|c|c|c|}
\hline \multirow[t]{2}{*}{ Source Term } & \multicolumn{5}{|c|}{ Population Case } \\
\hline & 1 & 2 & 5 & 6 & 7 \\
\hline RGG1 & 0.2 & $<0.1$ & 0.4 & 0.4 & 0.8 \\
\hline RGG2 & $<0.1$ & $<0.1$ & $<0.1$ & $<0.1$ & $<0.1$ \\
\hline RGG3 & $<0.1$ & $<0.1$ & $<0.1$ & $<0.1$ & $<0.1$ \\
\hline RGG4 & $<0.1$ & $<0.1$ & $<0.1$ & $<0.1$ & $<0.1$ \\
\hline RGG5 & 0 & 0 & 0 & 0 & 0 \\
\hline RLASI & $<0.1$ & $<0.1$ & $<0.1$ & $<0.1$ & $<0.1$ \\
\hline RLAS2 & $<0.1$ & $<0.1$ & $<0.1$ & $<0.1$ & $<0.1$ \\
\hline RLAS3 & $<0.1$ & $<0.1$ & $<0.1$ & $<0.1$ & $<0.1$ \\
\hline RLAS4 & $<0.1$ & $<0.1$ & $<0.1$ & $<0.1$ & $<0.1$ \\
\hline RLASS & $<0.1$ & $<0.1$ & 0.1 & 0.1 & 0.2 \\
\hline RLAS6 & $<0.1$ & $<0.1$ & $<0.1$ & $<0.1$ & $<0.1$ \\
\hline RLAS7 & $<0.1$ & $<0.1$ & $<0.1$ & $<0.1$ & $<0.1$ \\
\hline RPB1 & 0.1 & $<0.1$ & 0.2 & 0.2 & 0.4 \\
\hline RPB2 & $<0.1$ & $<0.1$ & 0.1 & 0.1 & 0.2 \\
\hline RPB3 & $<0.1$ & $<0.1$ & $<0.1$ & $<0.1$ & 0.1 \\
\hline RPB4 & $<0.1$ & $<0.1$ & $<0.1$ & 0 & $<0.1$ \\
\hline RPB5 & 0 & 0 & 0 & 0 & 0 \\
\hline RPB6 & 0.3 & $<0.1$ & 0.8 & 0.8 & 2 \\
\hline RSEQ1 & 62 & 13 & 120 & 120 & 240 \\
\hline RSEQ2 & 12 & 2 & 23 & 17 & 45 \\
\hline RSEQ3 & $<0.1$ & $<0.1$ & $<0.1$ & $<0.1$ & $<0.1$ \\
\hline RSEQ4 & 0 & 0 & 0 & 0 & 0 \\
\hline RSEQ5 & 23 & 5 & 46 & 41 & 92 \\
\hline
\end{tabular}


Table 4.2.1 (Continued)

\begin{tabular}{||l|c|c|c|c|c||}
\hline \multirow{2}{*}{ Source Term } & \multicolumn{5}{|c||}{ Population Case } \\
\cline { 2 - 6 } & 1 & 2 & 5 & 6 & 7 \\
\hline RSUR1 & 19 & 4 & 37 & 32 & 74 \\
\hline RSUR2 & 0.1 & $<0.01$ & 0.2 & 0.2 & 0.4 \\
\hline RSUR3 & 0 & 0 & 0 & 0 & 0 \\
\hline RSUR4 & 24 & 5 & 48 & 44 & 96 \\
\hline RZ1 & 25 & 5 & 49 & 46 & 97 \\
\hline RZ2 & $<0.1$ & $<0.1$ & $<0.1$ & $<0.1$ & $<0.1$ \\
\hline RZ3 & 0 & 0 & 0 & 0 & 0 \\
\hline RZ4 & 18 & 4 & 36 & 31 & 71 \\
\hline
\end{tabular}


Table 4.2.2 Changes to the Three Selected Source Terms to Effect One Early Fatality in the Mean as Estimated by MACCS

Emergency Response: Mean Evacuation

Exclusion Zone: 3 (0.4 mile)

Reactors All Scaled in Power to Effect One Early Fatality

80\% Weather with 3940 ft. Mixing Height

\begin{tabular}{|l|c|c|c|}
\hline Source Term & Original Power (MWth) & Adjusted Power (MWth) & Release Time \\
\hline RPB1 & 3800 & 9880 & Mid \\
\hline RPB6 & 3800 & 2926 & Mid \\
\hline RSUR2 & 3800 & 14000 & Late \\
\hline
\end{tabular}

Table 4.2.3 Mean Early Fatalities for the Three Source Terms, Initial Inventories Adjusted to Result in 1 Early Fatality for the Mean Evacuation.

\begin{tabular}{|c|c|c|c|}
\hline \multicolumn{4}{|c|}{ 0.4-1.4 miles } \\
\hline & Evacuees & Non-Evacuees & Total \\
\hline MPB1 & $0 * 0.995=0$ & $105 * 0.005=0.53$ & 0.53 \\
\hline MPB6 & $0 * 0.995=0$ & $107 * 0.005=0.54$ & 0.54 \\
\hline MSUR2 & $0 * 0.995=0$ & $118 * 0.005=0.59$ & 0.59 \\
\hline \multicolumn{4}{|c|}{$0.4-10$ miles } \\
\hline & Evacuees & Non-Evacuees & Total \\
\hline MPB1 & $0 * 0.995=0$ & $200 * 0.005=1.00$ & 1.00 \\
\hline MPB6 & $0 * 0.995=0$ & $200 * 0.005=1.00$ & 1.00 \\
\hline MSUR2 & $0 * 0.995=0$ & $200 * 0.005=1.00$ & 1.00 \\
\hline \multicolumn{4}{|c|}{ Population Weighted Early Fatalities 0.4-1.4 miles } \\
\hline & Evacuees & Non-Evacuees & Total \\
\hline MPB1 & $0 * 0.995=0$ & $0.0185 * 0.005=9.3 \mathrm{E}-5$ & 9.3E-5 \\
\hline MPB6 & $0 * 0.995=0$ & $0.0188 * 0.005=9.4 \mathrm{E}-5$ & $9.4 \mathrm{E}-5$ \\
\hline MSUR2 & $0 * 0.995=0$ & $0.02 * 0.005=1.0 \mathrm{E}-4$ & $1.0 \mathrm{E}-4$ \\
\hline
\end{tabular}


Table 4.2.4 Inventory Released and Shutdown Inventory by Individual Isotope for the Modified BWR Source Terms

\begin{tabular}{|c|c|c|c|c|c|c|}
\hline \multirow[t]{3}{*}{ Isotope } & \multirow[t]{3}{*}{ Group } & \multirow{3}{*}{$\begin{array}{l}\text { Shutdown } \\
\text { Inventory } \\
\text { (Ci) }\end{array}$} & \multicolumn{4}{|c|}{ Released Inventory } \\
\hline & & & \multicolumn{2}{|c|}{ MPB1 } & \multicolumn{2}{|c|}{ MPB6 } \\
\hline & & & (Ci) & Fraction & (Ci) & Fraction \\
\hline CO-58 & 6 & $5.8 \mathrm{E}+05$ & $2.5 E+03$ & $4.4 \mathrm{E}-03$ & $3.1 \mathrm{E}+03$ & $5.3 \mathrm{E}-03$ \\
\hline$c 0-60$ & 6 & $7.0 \mathrm{E}+05$ & $3.1 E+03$ & 4.4E-03 & $3.7 E+03$ & $5.4 \mathrm{E}-03$ \\
\hline KR-85 & 1 & $9.5 \mathrm{E}+05$ & $9.5 E+05$ & $1.0 \mathrm{E}+00$ & $6.9 E+05$ & $7.2 \mathrm{E}-01$ \\
\hline KR-85M & 1 & $3.5 E+07$ & $5.8 \mathrm{E}+06$ & $1.7 \mathrm{E}-01$ & $4.2 E+06$ & $1.2 \mathrm{E}-01$ \\
\hline KR-87 & 1 & $6.3 E+07$ & $1.1 \mathrm{E}+05$ & $1.8 \mathrm{E}-03$ & $8.2 E+04$ & $1.3 E-03$ \\
\hline KR-88 & 1 & $8.5 E+07$ & $4.9 E+06$ & $5.7 \mathrm{E}-02$ & $3.5 E+06$ & 4.2E-02 \\
\hline RB-86 & 3 & $5.3 E+04$ & $1.3 E+04$ & $2.4 \mathrm{E}-01$ & $1.5 \mathrm{E}+04$ & $2.8 \mathrm{E}-01$ \\
\hline SR-89 & 5 & $1.1 \mathrm{E}+08$ & $1.1 \mathrm{E}+07$ & $1.0 \mathrm{E}-01$ & $1.2 E+07$ & $1.2 \mathrm{E}-01$ \\
\hline SR-90 & 5 & $7.5 E+06$ & $7.5 E+05$ & $1.0 \mathrm{E}-01$ & $8.9 E+05$ & $1.2 \mathrm{E}-01$ \\
\hline SR-91 & 5 & $1.4 \mathrm{E}+08$ & $5.8 E+06$ & $4.3 E-02$ & $6.9 E+06$ & 5.0E-02 \\
\hline SR-92 & 5 & $1.4 \mathrm{E}+08$ & $7.1 \mathrm{E}+05$ & $5.0 \mathrm{E}-03$ & $8.4 E+05$ & $5.9 \mathrm{E}-03$ \\
\hline Y-90 & 7 & $8.0 \mathrm{E}+06$ & $1.5 \mathrm{E}+05$ & $1.8 \mathrm{E}-02$ & $1.6 \mathrm{E}+05$ & $2.0 \mathrm{E}-02$ \\
\hline Y-91 & 7 & $1.3 E+08$ & $1.1 E+06$ & $8.4 \mathrm{E}-03$ & $1.1 E+06$ & $8.6 \mathrm{E}-03$ \\
\hline Y-92 & 7 & $1.4 \mathrm{E}+08$ & $2.5 E+06$ & $1.7 \mathrm{E}-02$ & $2.9 \mathrm{E}+06$ & $2.0 \mathrm{E}-02$ \\
\hline Y-93 & 7 & $1.6 \mathrm{E}+08$ & $5.9 E+05$ & $3.6 \mathrm{E}-03$ & $5.9 \mathrm{E}+05$ & $3.6 \mathrm{E}-03$ \\
\hline ZR-95 & 7 & $1.7 \mathrm{E}+08$ & $1.4 \mathrm{E}+06$ & $8.0 E-03$ & $1.4 \mathrm{E}+06$ & 8.1E-03 \\
\hline ZR-97 & 7 & $1.7 \mathrm{E}+08$ & $8.7 E+05$ & $5.0 \mathrm{E}-03$ & $8.7 E+05$ & $5.0 \mathrm{E}-03$ \\
\hline NB-95 & 7 & $1.6 \mathrm{E}+08$ & $1.3 E+06$ & $8.1 E-03$ & $1.3 \mathrm{E}+06$ & 8.1E-03 \\
\hline MO-99 & 6 & $1.8 \mathrm{E}+08$ & $7.2 E+05$ & $3.9 E-03$ & $8.8 E+05$ & $4.8 E-03$ \\
\hline TC-99M & 6 & $1.6 \mathrm{E}+08$ & $7.4 E+05$ & 4.7E-03 & $9.0 \mathrm{E}+05$ & $5.7 \mathrm{E}-03$ \\
\hline RU-103 & 6 & $1.4 \mathrm{E}+08$ & $6.1 E+05$ & $4,4 E-03$ & $7.4 \mathrm{E}+05$ & $5.3 \mathrm{E}-03$ \\
\hline RU-105 & 6 & $9.3 E+07$ & $6.8 E+04$ & 7.2E-04 & $8.2 E+04$ & $8.8 E-04$ \\
\hline RU-106 & 6 & $3.8 \mathrm{E}+07$ & $1.7 \mathrm{E}+05$ & 4.4E-03 & $2.0 \mathrm{E}+05$ & $5.4 \mathrm{E}-03$ \\
\hline RH-105 & 6 & $7.0 \mathrm{E}+07$ & $2.8 \mathrm{E}+05$ & $4.0 E-03$ & $3.4 \mathrm{E}+05$ & 4.9E-03 \\
\hline SB-127 & 4 & $8.8 E+06$ & $1.4 \mathrm{E}+06$ & $1.6 \mathrm{E}-01$ & $1.7 \mathrm{E}+06$ & $1.9 \mathrm{E}-01$ \\
\hline SB-129 & 4 & $3.1 \mathrm{E}+07$ & $8.4 \mathrm{E}+05$ & 2.7E-02 & $9.9 \mathrm{E}+05$ & $3.2 \mathrm{E}-02$ \\
\hline TE-127 & 4 & $8.6 E+06$ & $1.5 \mathrm{E}+06$ & $1.8 \mathrm{E}-01$ & $1.8 E+06$ & $2.1 E-01$ \\
\hline TE-127M & 4 & $1.2 \mathrm{E}+06$ & $2.1 E+05$ & $1.8 \mathrm{E}-01$ & $2.4 \mathrm{E}+05$ & 2.1E-01 \\
\hline TE-129 & 4 & $2.9 E+07$ & $1.1 \mathrm{E}+06$ & $4.0 \mathrm{E}-02$ & $1.4 \mathrm{E}+06$ & $4.7 \mathrm{E}-02$ \\
\hline
\end{tabular}


4 Results

Table 4.2.4 (Continued)

\begin{tabular}{|c|c|c|c|c|c|c|}
\hline \multirow[t]{3}{*}{ Isotope } & \multirow[t]{3}{*}{ Group } & \multirow{3}{*}{$\begin{array}{l}\text { Shutdown } \\
\text { Inventory } \\
\text { (Ci) }\end{array}$} & \multicolumn{4}{|c|}{ Released Inventory } \\
\hline & & & \multicolumn{2}{|c|}{ MPB1 } & \multicolumn{2}{|c|}{ MPB6 } \\
\hline & & & (Ci) & Fraction & (Ci) & Fraction \\
\hline TE-129M & 4 & $7.6 \mathrm{E}+06$ & $1.3 E+06$ & $1.8 \mathrm{E}-01$ & $1.6 \mathrm{E}+06$ & 2.1E-01 \\
\hline TE-131M & 4 & $1.5 \mathrm{E}+07$ & $2.0 \mathrm{E}+06$ & $1.4 \mathrm{E}-01$ & $2.3 E+06$ & $1.6 \mathrm{E}-01$ \\
\hline TE-132 & 4 & $1.4 \mathrm{E}+08$ & $2.3 E+07$ & $1.6 \mathrm{E}-01$ & $2.7 \mathrm{E}+07$ & $1.9 \mathrm{E}-01$ \\
\hline $\mathrm{I}-131$ & 2 & $9.8 \mathrm{E}+07$ & $2.5 E+07$ & $2.6 \mathrm{E}-01$ & $3.0 \mathrm{E}+07$ & $3.1 E-01$ \\
\hline $\mathrm{I}-132$ & 2 & $1.4 \mathrm{E}+08$ & $2.4 \mathrm{E}+07$ & $1.7 \mathrm{E}-01$ & $2.8 \mathrm{E}+07$ & $2.0 \mathrm{E}-01$ \\
\hline I-133 & 2 & $2.1 E+08$ & $3.7 \mathrm{E}+07$ & $1.8 \mathrm{E}-01$ & $4.4 \mathrm{E}+07$ & $2.2 \mathrm{E}-01$ \\
\hline I-134 & 2 & $2.3 E+08$ & $5.7 \mathrm{E}+03$ & $2.5 \mathrm{E}-05$ & $6.8 E+03$ & $3.0 \mathrm{E}-05$ \\
\hline I-135 & 2 & $1.9 E+08$ & $1.5 \mathrm{E}+07$ & 7.8E-02 & $1.8 \mathrm{E}+07$ & $9.3 \mathrm{E}-02$ \\
\hline XE-133 & 1 & $2.1 E+08$ & $2.0 \mathrm{E}+08$ & $9.6 \mathrm{E}-01$ & $1.4 \mathrm{E}+08$ & $7.0 \mathrm{E}-01$ \\
\hline $\mathrm{XE}-135$ & 1 & $4.9 E+07$ & $3.7 \mathrm{E}+07$ & $7.5 \mathrm{E}-01$ & $3.4 \mathrm{E}+07$ & $6.9 E-01$ \\
\hline CS-134 & 3 & $1.6 \mathrm{E}+07$ & $3.9 E+06$ & 2.4E-01 & $4.7 \mathrm{E}+06$ & $2.9 \mathrm{E}-01$ \\
\hline CS-136 & 3 & $4.3 \mathrm{E}+06$ & $1.0 \mathrm{E}+06$ & 2.4E-01 & $1.2 \mathrm{E}+06$ & $2.8 \mathrm{E}-01$ \\
\hline CS-137 & 3 & $9.6 \mathrm{E}+06$ & $2.4 E+06$ & 2.4E-01 & $2.8 \mathrm{E}+06$ & $2.9 \mathrm{E}-01$ \\
\hline BA-139 & 9 & $1.9 \mathrm{E}+08$ & $4.1 E+04$ & $2.1 \mathrm{E}-04$ & $4.8 \mathrm{E}+04$ & $2.5 \mathrm{E}-04$ \\
\hline BA-140 & 9 & $1.9 \mathrm{E}+08$ & $1.4 \mathrm{E}+07$ & $7.4 \mathrm{E}-02$ & $1.7 \mathrm{E}+07$ & $8.8 \mathrm{E}-02$ \\
\hline iA-140 & 7 & $1.9 E+08$ & $3.8 \mathrm{E}+06$ & 2.0E-02 & $4.3 E+06$ & $2.3 E-02$ \\
\hline LA-141 & 7 & $1.8 \mathrm{E}+08$ & $1.8 \mathrm{E}+05$ & 1.0E-03 & $1.8 \mathrm{E}+05$ & $1.0 \mathrm{E}-03$ \\
\hline LA-142 & 7 & $1.7 \mathrm{E}+08$ & $8.3 E+03$ & 4.9E-05 & $8.3 E+03$ & $4.9 E-05$ \\
\hline CE-141 & 8 & $1.7 E+08$ & $1.8 \mathrm{E}+06$ & $1.0 \mathrm{E}-02$ & $2.6 \mathrm{E}+06$ & $1.5 \mathrm{E}-02$ \\
\hline CE-143 & 8 & $1.7 \mathrm{E}+08$ & $1.4 \mathrm{E}+06$ & 8.2E-03 & $2.0 \mathrm{E}+06$ & 1.2E-02 \\
\hline CE-144 & 8 & $1.1 \mathrm{E}+08$ & $1.2 E+06$ & $1.1 E-02$ & $1.7 E+06$ & $1.5 E-02$ \\
\hline PR-143 & 7 & $1.6 \mathrm{E}+08$ & $1.3 E+06$ & 8.1E-03 & $1.3 E+06$ & $8.3 E-03$ \\
\hline ND-147 & 7 & $7.2 \mathrm{E}+07$ & $5.7 \mathrm{E}+05$ & $7.8 \mathrm{E}-03$ & $5.7 \mathrm{E}+05$ & $7.9 \mathrm{E}-03$ \\
\hline NP-239 & 8 & $2.2 \mathrm{E}+09$ & $2.0 \mathrm{E}+07$ & $9.1 \mathrm{E}-03$ & $2.9 \mathrm{E}+07$ & $1.3 \mathrm{E}-02$ \\
\hline PU-238 & 8 & $1.5 \mathrm{E}+05$ & $1.6 \mathrm{E}+03$ & $1.1 \mathrm{E}-02$ & $2.3 E+03$ & $1.5 \mathrm{E}-02$ \\
\hline PU-239 & 8 & $3.8 \mathrm{E}+04$ & $4.0 \mathrm{E}+02$ & $1.1 \mathrm{E}-02$ & $5.8 \mathrm{E}+02$ & $1.5 \mathrm{E}-02$ \\
\hline PU-240 & 8 & $4.8 \mathrm{E}+04$ & $5.0 \mathrm{E}+02$ & $1.1 \mathrm{E}-02$ & $7.3 E+02$ & $1.5 E-02$ \\
\hline PU-241 & 8 & $8.2 \mathrm{E}+06$ & $8.6 E+04$ & $1.1 \mathrm{E}-02$ & $1.3 E+05$ & $1.5 \mathrm{E}-02$ \\
\hline AM-241 & 7 & $8.3 E+03$ & $6.7 E+01$ & 8.1E-03 & $6.8 \mathrm{E}+01$ & 8.1E-03 \\
\hline CM-242 & 7 & $2.2 E+06$ & $1.8 \mathrm{E}+04$ & 8.1E-03 & $1.8 E+04$ & 8.1E-03 \\
\hline CM-244 & 7 & $1.2 E+05$ & $9.6 \mathrm{E}+02$ & 8.1E-03 & $9.7 \mathrm{E}+02$ & 8.1E-03 \\
\hline
\end{tabular}


Table 4.2.5 Inventory Released and Shutdown Inventory by Isotope Groups for the Two BWR Source Terms

\begin{tabular}{|c|c|c|c|c|c|}
\hline \multirow[t]{3}{*}{ Group } & \multirow{3}{*}{$\begin{array}{c}\text { Shutdown } \\
\text { Inventory } \\
\text { (Ci) }\end{array}$} & \multicolumn{4}{|c|}{ Released Inventory } \\
\hline & & \multicolumn{2}{|c|}{ MPB1 } & \multicolumn{2}{|c|}{ MPB6 } \\
\hline & & $(\mathrm{Ci})$ & Fraction & (Ci) & Fraction \\
\hline 1 & $4.4 \mathrm{E}+08$ & $2.5 E+08$ & $5.6 E-01$ & $1.9 E+08$ & $4.2 E-01$ \\
\hline 2 & $8.7 E+08$ & $1.0 \mathrm{E}+08$ & $1.2 \mathrm{E}-01$ & $1.2 E+08$ & $1.4 E-01$ \\
\hline 3 & $3.0 \mathrm{E}+07$ & $7.3 E+06$ & $2.4 E-01$ & $8.7 E+06$ & $2.9 \mathrm{E}-01$ \\
\hline 4 & $2.4 E+08$ & $3.1 \mathrm{E}+07$ & $1.3 E-01$ & $3.7 \mathrm{E}+07$ & $1.5 \mathrm{E}-01$ \\
\hline 5 & $3.9 \mathrm{E}+08$ & $1.8 E+07$ & $4.5 E-02$ & $2.1 E+07$ & $5.4 E-02$ \\
\hline 6 & $6.9 E+08$ & $2.6 E+06$ & $3.8 E-03$ & $3.2 E+06$ & 4.6E-03 \\
\hline 7 & $1.7 \mathrm{E}+09$ & $1.4 E+07$ & $8.0 E-03$ & $1.5 E+07$ & 8.6E-03 \\
\hline 8 & $2.6 E+09$ & $2.4 \mathrm{E}+07$ & $9.2 E-03$ & $3.5 E+07$ & 1.3E-02 \\
\hline 9 & $3.8 E+08$ & $1.4 \mathrm{E}+07$ & 3.7E-02 & $1.7 E+07$ & 4.4E-02 \\
\hline
\end{tabular}


Table 4.2.6 Inventory Released and Shutdown Inventory by Individual Isotope for the Modified PWR Source Term

\begin{tabular}{|c|c|c|c|c|}
\hline \multirow[t]{3}{*}{ Isotope } & \multirow[t]{3}{*}{ Group } & \multirow{3}{*}{$\begin{array}{l}\text { Shutdown Inventory } \\
\text { (Ci) }\end{array}$} & \multirow{2}{*}{\multicolumn{2}{|c|}{$\begin{array}{c}\text { Released Inventory } \\
\text { MSUR2 }\end{array}$}} \\
\hline & & & & \\
\hline & & & (Ci) & Fraction \\
\hline CO-58 & 6 & $9.7 \mathrm{E}+05$ & $2.6 \mathrm{E}+03$ & 2.7E-03 \\
\hline$C O-60$ & 6 & $7.4 E+05$ & $2.0 \mathrm{E}+03$ & $2.7 \mathrm{E}-03$ \\
\hline KR-85 & 1 & $7.4 E+05$ & $7.6 \mathrm{E}+05$ & $1.0 \mathrm{E}+00$ \\
\hline KR-85M & 1 & $3.5 E+07$ & $5.6 \mathrm{E}+06$ & $1.6 \mathrm{E}-01$ \\
\hline KR-87 & 1 & $6.4 E+07$ & $9.2 \mathrm{E}+04$ & $1.4 E-03$ \\
\hline KR-88 & 1 & $8.6 E+07$ & $4.5 E+06$ & 5.2E-02 \\
\hline RB-86 & 3 & $5.7 \mathrm{E}+04$ & $4.5 E+03$ & $7.9 \mathrm{E}-02$ \\
\hline SR-89 & 5 & $1.1 E+08$ & $8.7 E+05$ & 8.0E-03 \\
\hline SR-90 & 5 & $5.8 \mathrm{E}+06$ & $4.7 E+04$ & 8.1E-03 \\
\hline SR-91 & 5 & $1.4 E+08$ & $4.7 E+05$ & $3.4 \mathrm{E}-03$ \\
\hline SR-92 & 5 & $1.4 E+08$ & $5.4 \mathrm{E}+04$ & 3.8E-0.4 \\
\hline$Y-90$ & 7 & $6.3 E+06$ & $1.2 \mathrm{E}+04$ & $1.9 E-03$ \\
\hline$Y-91$ & 7 & $1.3 E+08$ & $1.5 \mathrm{E}+05$ & $1.1 \mathrm{E}-03$ \\
\hline$Y-92$ & 7 & $1.5 \mathrm{E}+08$ & $2.0 \mathrm{E}+05$ & $1.4 E-03$ \\
\hline$Y-93$ & 7 & $1.6 E+08$ & $7.8 \mathrm{E}+04$ & 4.7E-04 \\
\hline ZR-95 & 7 & $1.7 E+08$ & $1.8 \mathrm{E}+05$ & $1.1 \mathrm{E}-03$ \\
\hline ZR-97 & 7 & $1.7 E+08$ & $1.1 \mathrm{E}+05$ & $6.6 E-04$ \\
\hline NB-95 & 7 & $1.6 \mathrm{E}+08$ & $1.7 E+05$ & 1.1E-03 \\
\hline MO-99 & 6 & $1.8 E+08$ & $4.4 E+05$ & $2.4 \mathrm{E}-03$ \\
\hline TC-99M & 6 & $1.6 \mathrm{E}+08$ & $4.5 E+05$ & $2.8 \mathrm{E}-03$ \\
\hline RU-103 & 6 & $1.4 E+08$ & $.3 .6 \mathrm{E}+05$ & 2.7E-03 \\
\hline RU-105 & 6 & $8.9 E+07$ & $3.7 \mathrm{E}+04$ & 4.1E-04 \\
\hline RU-106 & 6 & $3.1 E+07$ & $8.4 E+04$ & 2.7E-03 \\
\hline RH-105 & 6 & $6.2 \mathrm{E}+07$ & $1.5 \mathrm{E}+05$ & $2.5 \mathrm{E}-03$ \\
\hline SB-127 & 4 & $8.4 E+06$ & $1.9 \mathrm{E}+06$ & $2.2 \mathrm{E}-01$ \\
\hline SB-129 & 4 & $3.0 \mathrm{E}+07$ & $1.1 E+06$ & $3.6 \mathrm{E}-02$ \\
\hline TE-127 & 4 & $8.1 E+06$ & $1.9 \mathrm{E}+06$ & $2.4 \mathrm{E}-01$ \\
\hline TE-127M & 4 & $1.1 E+06$ & $2.6 \mathrm{E}+05$ & 2.4E-01 \\
\hline TE-129 & 4 & $2.8 E+07$ & $1.4 \mathrm{E}+06$ & $5.2 \mathrm{E}-02$ \\
\hline
\end{tabular}


Table 4.2.6 (Continued)

\begin{tabular}{|c|c|c|c|c|}
\hline \multirow[t]{3}{*}{ Isotope } & \multirow[t]{3}{*}{ Group } & \multirow{3}{*}{$\begin{array}{l}\text { Shutdown Inventory } \\
\text { (Ci) }\end{array}$} & \multirow{2}{*}{\multicolumn{2}{|c|}{$\frac{\text { Released Inventory }}{\text { MSUR2 }}$}} \\
\hline & & & & \\
\hline & & & (Ci) & Fraction \\
\hline TE-129M & 4 & $7.4 \mathrm{E}+06$ & $1.8 \mathrm{E}+06$ & $2.4 \mathrm{E}-01$ \\
\hline TE-131M & 4 & $1.4 \mathrm{E}+07$ & $2.6 \mathrm{E}+06$ & $1.8 \mathrm{E}-01$ \\
\hline TE-132 & 4 & $1.4 \mathrm{E}+08$ & $3.1 E+07$ & 2.2E-01 \\
\hline I-131 & 2 & $9.7 \mathrm{E}+07$ & $1.5 \mathrm{E}+07$ & $1.6 \mathrm{E}-01$ \\
\hline I-132 & 2 & $1.4 \mathrm{E}+08$ & $3.1 E+07$ & $2.2 \mathrm{E}-01$ \\
\hline I-133 & 2 & $2.0 \mathrm{E}+08$ & $2.2 \mathrm{E}+07$ & $1.1 \mathrm{E}-01$ \\
\hline I-134 & 2 & $2.2 \mathrm{E}+08$ & $2.7 \mathrm{E}+03$ & $1.2 \mathrm{E}-05$ \\
\hline I-135 & 2 & $1.9 E+08$ & $8.8 \mathrm{E}+06$ & $4.6 \mathrm{E}-02$ \\
\hline XE-133 & 1 & $2.0 \mathrm{E}+08$ & $2.0 \mathrm{E}+08$ & $9.7 \mathrm{E}-01$ \\
\hline XE-135 & 1 & $3.8 \mathrm{E}+07$ & $2.5 E+07$ & $6.6 \mathrm{E}-01$ \\
\hline CS-134 & 3 & $1.3 \mathrm{E}+07$ & $1.1 \mathrm{E}+06$ & 8.1E-02 \\
\hline CS-136 & 3 & $4.0 \mathrm{E}+06$ & $3.1 \mathrm{E}+05$ & $7.8 \mathrm{E}-02$ \\
\hline CS-137 & 3 & $7.3 E+06$ & $5.9 E+05$ & 8.1E-02 \\
\hline BA-139 & 9 & $1.9 E+08$ & $3.8 \mathrm{E}+03$ & $2.0 \mathrm{E}-05$ \\
\hline BA-140 & 9 & $1.9 E+08$ & $1.5 E+06$ & $7.9 \mathrm{E}-03$ \\
\hline LA-140 & 7 & $1.9 E+08$ & $4.5 E+05$ & $2.3 \mathrm{E}-03$ \\
\hline LA-141 & 7 & $1.8 \mathrm{E}+08$ & $2.3 E+04$ & $1.3 E-04$ \\
\hline LA-142 & 7 & $1.7 \mathrm{E}+08$ & $9.7 \mathrm{E}+02$ & $5.8 \mathrm{E}-06$ \\
\hline CE-141 & 8 & $1.7 \mathrm{E}+08$ & $1.8 \mathrm{E}+05$ & $1.1 \mathrm{E}-03$ \\
\hline CE-143 & 8 & $1.7 E+08$ & $1.4 \mathrm{E}+05$ & 8.4E-04 \\
\hline CE-144 & 8 & $1.0 \mathrm{E}+08$ & $1.1 \mathrm{E}+05$ & $1.1 \mathrm{E}-03$ \\
\hline PR-143 & 7 & $1.6 E+08$ & $1.7 E+05$ & $1.1 \mathrm{E}-03$ \\
\hline ND-147 & 7 & $7.3 E+07$ & $7.6 \mathrm{E}+04$ & $1.0 \mathrm{E}-03$ \\
\hline NP-239 & 8 & $1.9 \mathrm{E}+09$ & $1.8 \mathrm{E}+06$ & $9.3 \mathrm{E}-04$ \\
\hline PU-238 & 8 & $1.1 \mathrm{E}+05$ & $1.2 \mathrm{E}+02$ & $1.1 \mathrm{E}-03$ \\
\hline PU-239 & 8 & $2.5 E+04$ & $2.7 \mathrm{E}+01$ & $1.1 \mathrm{E}-03$ \\
\hline PU-240 & 8 & $3.1 E+04$ & $3.4 \mathrm{E}+01$ & $1.1 \mathrm{E}-03$ \\
\hline PU-241 & 8 & $5.3 E+06$ & $5.7 E+03$ & $1.1 \mathrm{E}-03$ \\
\hline AM-241 & 7 & $3.5 E+03$ & $3.8 \mathrm{E}+\infty 0$ & $1.1 \mathrm{E}-03$ \\
\hline CM-242 & 7 & $1.3 E+06$ & $1.4 \mathrm{E}+03$ & $1.1 \mathrm{E}-03$ \\
\hline CM-244 & 7 & $7.8 \mathrm{E}+04$ & $8.4 \mathrm{E}+01$ & $1.1 \mathrm{E}-03$ \\
\hline
\end{tabular}


4 Results

Table 4.2.7 Inventory Released and Shutdown by lsotope Groups for the Modified PWR Source Term

\begin{tabular}{|c|c|c|c|}
\hline \multirow{2}{*}{$\begin{array}{c}\text { Isotope } \\
\text { Group }\end{array}$} & \multirow{2}{*}{$\begin{array}{l}\text { Shutdown } \\
\text { Inventory }\end{array}$} & & \multicolumn{2}{|c|}{ Released Inventory } \\
\cline { 3 - 4 } & & $(\mathrm{C})$ & MSUR2 \\
\cline { 3 - 4 } & & $2.3 \mathrm{E}+08$ & $5.5 \mathrm{E}-01$ \\
\hline 1 & $4.3 \mathrm{E}+08$ & $7.7 \mathrm{E}+07$ & $9.0 \mathrm{E}-02$ \\
\hline 2 & $8.6 \mathrm{E}+08$ & $2.0 \mathrm{E}+06$ & $8.0 \mathrm{E}-02$ \\
\hline 3 & $2.4 \mathrm{E}+07$ & $4.1 \mathrm{E}+07$ & $1.8 \mathrm{E}-01$ \\
\hline 4 & $2.4 \mathrm{E}+08$ & $1.4 \mathrm{E}+06$ & $3.6 \mathrm{E}-03$ \\
\hline 5 & $4.0 \mathrm{E}+08$ & $1.5 \mathrm{E}+06$ & $2.3 \mathrm{E}-03$ \\
\hline 6 & $6.6 \mathrm{E}+08$ & $1.6 \mathrm{E}+06$ & $9.1 \mathrm{E}-04$ \\
\hline 7 & $1.8 \mathrm{E}+09$ & $2.2 \mathrm{E}+06$ & $9.4 \mathrm{E}-04$ \\
\hline 8 & $2.4 \mathrm{E}+09$ & $1.5 \mathrm{E}+06$ & $3.9 \mathrm{E}-03$ \\
\hline 9 & $3.8 \mathrm{E}+08$ & & \\
\hline
\end{tabular}


Table 4.2.8 Early Fatalities for the Three Adjusted Source Terms

(This table illustrates the contributions to the early fatalities. The first number (multiplied by 0.995 ) is the number of early fatalities incurred by the evacuees, the second set (multiplied by 0.005 ) is the number of early fatalities incurred by the non-evacuees, the last number is the total number of early fatalities.)

\begin{tabular}{|l|c|c|c|c|}
\hline \multicolumn{5}{|c|}{ Total Early Fatalities 0.4-1.4 Miles } \\
\hline & Evacuees & Non-Evacuees & & Total \\
\hline RPB1 & $0 * 0.995$ & $+105 * 0.005$ & $=$ & 0.526 \\
\hline RPB6 & $0 * 0.995$ & $+107 * 0.005$ & $=$ & 0.535 \\
\hline RSUR2 & $0 * 0.995$ & $+118 * 0.005$ & $=$ & 0.589 \\
\hline
\end{tabular}


4 Results

Table 4.2.9 Estimated Number of Individuals Receiving Exposure Exceeding Threshold Doses to the Three Critical Organs in the Early Fatality Health Effects Model

\begin{tabular}{|c|c|c|c|c|c|}
\hline & $\begin{array}{c}\text { Contribution from } \\
\text { Evacuees } \\
99.5 \%\end{array}$ & & $\begin{array}{c}\text { Contribution from } \\
\text { Non-Evacuees } \\
0.5 \%\end{array}$ & & $\begin{array}{l}\text { Mean Number of } \\
\text { Individuals }\end{array}$ \\
\hline \multicolumn{6}{|l|}{ Source Term: RPB1 } \\
\hline Red Marrow > 150 Rem & $0.995 * 0$ & + & $0.005 * 612$ & $=$ & 3.1 \\
\hline Lungs > 500 Rem & $0.995 * 0$ & + & $0.005 * 284$ & $=$ & 1.4 \\
\hline Lower LI > 800 Rem & $0.995 * 0$ & + & $0.005 * 143$ & $=$ & 0.7 \\
\hline \multicolumn{6}{|l|}{ Source Term: RPB6 } \\
\hline Red Marrow > 150 Rem & $0.995 * 0$ & + & $0.005 * 834$ & $=$ & 4.2 \\
\hline Lungs > 500 Rem & $0.995 * 0$ & + & $0.005 * 340$ & $=$ & 1.7 \\
\hline Lower LI > 800 Rem & $0.995 * 0$ & + & $0.005 * 125$ & $=$ & 0.6 \\
\hline \multicolumn{6}{|l|}{ Source Term: RSUR2 } \\
\hline Red Marrow > 150 Rem & $0.995 * 0$ & + & $0.005 * 594$ & $=$ & 3.0 \\
\hline Lungs > 500 Rem & $0.995 * 0$ & + & $0.005 * 215$ & $=$ & 1.1 \\
\hline Lower LI > 800 Rem & $0.995 * 0$ & + & $0.005 * 83$ & $=$ & 0.4 \\
\hline
\end{tabular}




\subsection{Source Terms for the Conservative Evacuation Case}

The next set of large release source terms considered was for the conservative evacuation case. By inspection of the survey results for the no evacuation and the mean evacuation cases, three source terms (RPB2, RPB3, and RSUR2) were identified as candidates. For each of these source terms the release fractions for the iodine, cesium, and tellurium groups were then arbitrarily adjusted until MACCS predicted one mean early fatality. The estimated number of early fatalities are presented in Table 4.3.1. The release time classifications are also given in the table based on the discussion in Section 4.2. For the conservative evacuation case, the potential early fatality in the mid release time category source term is dominated by the population that is evacuated. For the late release source term, the sole contribution to the MACCS predicted early fatality is from the population that did not participate in the evacuation. In the conservative evacuation case, there is an order of magnitude increase in the number of people who did not participate in the evacuation, as compared to the mean evacuation case. The modified releases are small enough that there was little contribution to the estimated number of early fatalities one mile beyond the exclusion boundary.

Table 4.3.2 shows the physical characteristics of the source terms before and after the modification required to achieve the result of one early fatality predicted by MACCS. Inspection of the table shows that for the mid release time source terms, the I release fraction had to be reduced by approximately a third; the Cs and Te fractions noeded small reductions.

Tables 4.3.3 and 4.3.4 provide the inventories and fractions of individual isotopes released from containment, for the modified BWR and PWR source terms, respectively. Tables 4.3.5 and 4.3.6 show the releases by the standard isotope groupings.

Because the population that participated in the evacuation in the conservative evacuation case contributes to the early fatalities for the two mid release timings, there should be different dose pathways for the exposures, when compared to the mean evacuation case. Table 4.3.7 shows the total number of cases of exposure exceeding the early fatality threshold doses to the three critical organs (red marrow, lungs, lower large intestine) considered in the early fatality health effects model in MACCS.

Tables B.3.1-B.3.3 of Appendix B provide the centerline dose from the modified source term MPB3 to red marrow, lungs and lower large intestine as a function of distance and for the different pathways that make up the acute (seven day) exposure. The dose to the evacuees is now large compared to the dose to the non-evacuees. The main contributor to the red marrow dose, which is the major contributor to the early fatality, was from inhalation. The lung and large intestine received major doses from inhalation, with the groundshine contribution being about $60 \%$ of the inhalation contribution.

Also in Appendix B, Tables B.3.4-B.3-6 provide the relative fraction of dose at each distance, for each pathway and by protective action assumption. Again the red marrow dose is the dominant contributor to the potential early fatalities. For those participating in the evacuation, cloudshine and inhalation were the dominant pathways for dose, with inhalation most dominant close in (0.4-1.4 miles). This close in region is where MACCS estimates the greatest potential for early fatalities to occur. However, there was some contribution ( $>20 \%)$ from groundshine. In the non-evacuation population, the groundshine was the largest factor, with cloudshine and inhalation making significant contributions. Dose to the lungs and large intestines was dominated by inhalation in both groups. Cloudshine was the next largest contributor for the evacuees, and groundshine was the second largest contributor for the nonevacuees. 


\section{Results}

The potential to lead to an early fatality is linearly dependent on the population density, for the population cases 1, 5 and 7 where population was uniformly distributed from the exclusion boundary out to 30 miles. This results from the way the MACCS codes estimates early fatalities. The MACCS codes first estimates the individual risks of early fatality and then multiplies the results by the affected populations. However, since the individual risk model is nonlinear with dose, the radioisotope releases that have the potential to lead to one early fatality will not scale linearly with uniform population density. To examine the impact of the non-linearity of the early fatality risk model on the magnitude of the radionuclide releases, a set of four calculations were done. In these calculations the population surrounding the plant was assumed to be uniformly distributed out to 30 miles and had a density of 10 people per square mile. Four source terms, two BWR source terms, RPB1 and RPB6, and two PWR source terms, RSEQ1 and RSEQ5 were selected for modification in this case. For each of these source terms the release fractions of the iodine, cesium, and tellurium groups were adjusted so as to lead to approximately one MACCS predicted mean early fatality. Table 4.3.8 shows the MACCS predicted early fatalities for each of the modified source terms by the evacuee and non-evacuee populations and the release time categories. Since the conservative evacuation response is assumed for this calculation, both the evacuee and the non-evacuee populations contribute to the calculatod number of early fatalities. Table 4.3.9 displays the modifications needed to the release fraction of the I, Cs, and Te groups of the original source terms to arrive at a MACCS predicted one mean early fatality.

The inventories of nuclides released to the environment by the modified BWR source terms are listed in Table 4.3.10, and the inventories released by the modified PWR source terms are listed in Table 4.3.11. Grouped radioisotope releases for the modified BWR and PWR source terms are provided in Tables 4.3.12 and 4.3.13, respectively. 
Table 4.3.1 Estimated Mean Early Fatalities for the Three Modified Source Terms

Emergency Response: Conservative Evacuation Case

Population Distribution: Uniform 1000 People/Sq. Mi.

Exclusion Zone Boundary: 3 (0.4 mile)

80\% Weather with 3940 ft. Mixing Height

\begin{tabular}{|c|c|c|c|}
\hline \multicolumn{4}{|c|}{$0.4-1.4$ miles } \\
\hline & Evacuees & Non-Evacues & Total \\
\hline MPB2 (Mid) & $0.65 * .95=0.62$ & $6.5 * .05=0.325$ & 0.945 \\
\hline MPB3 (Mid) & $0.78 * .95=0.74$ & $3.8 * .05=0.19$ & 0.933 \\
\hline MSUR2 (Lato) & $0 * .95=0$ & $21 . * .05=1.04$ & 1.04 \\
\hline \multicolumn{4}{|c|}{ Population Weighted Early Fatalities } \\
\hline \multicolumn{4}{|c|}{$0.4-1.4$ miles } \\
\hline & Evacuees & Non-Evacuees & Total \\
\hline MPB2 (Mid) & $6.1 E-4 * 0.95=5.8 E-4$ & $3.8 \mathrm{E}-3 * 0.05=1.9 \mathrm{E}-4$ & $7.7 E-4$ \\
\hline MPB3 (Mid) & $2.3 \mathrm{E}-4 * 0.95=2.2 \mathrm{E}-4$ & $1.1 \mathrm{E}-3 * 0.05=5.5 \mathrm{E}-5$ & $2.8 E-4$ \\
\hline MSUR2 (Late) & $0.0 * 0.95=0.0$ & $3.6 \mathrm{E}-3 * 0.05=1.8 \mathrm{E}-4$ & $1.8 E-4$ \\
\hline
\end{tabular}


Table 4.3.2 Original and Modified Release Fractions

Emergency Response: Conservative Evacuation Case

Population Distribution: Uniform 1000 People/Sq. Mi.

Exclusion Zone Boundary: 3 (0.4 mile)

80\% Weather with $3940 \mathrm{ft}$. Mixing Height

\section{Original Release Fractions}

\begin{tabular}{|c|c|c|c|c|c|c|c|c|c|}
\hline $\begin{array}{l}\text { Source } \\
\text { Term }\end{array}$ & $\mathbf{X E} / \mathbf{K R}$ & I & CS & $\mathbf{T E}$ & SR & $\mathbf{R U}$ & $\mathbf{L A}$ & CE & $\mathbf{B A}$ \\
\hline \multicolumn{10}{|c|}{ RPB3 (MID)a: } \\
\hline PUFF1 & 0.76 & 2.3E-3 & 0.001 & $1.5 \mathrm{E}-3$ & $5 . E-4$ & S.E-5 & 1.E-5 & 5.E-5 & $6 . E-4$ \\
\hline PUFF2 & 0.24 & 0.04 & 0.04 & 0.03 & 0.02 & $3 . E-4$ & $1.5 \mathrm{E}-3$ & 2.E-3 & 0.02 \\
\hline \multicolumn{10}{|c|}{ RPB2 (MID)": } \\
\hline PUFF1 & 0.76 & 0.01 & 0.01 & 3.E-3 & 1.E-3 & $1.2 \mathrm{E}-3$ & 3.E-4 & 3.E-4 & $1.2 \mathrm{E}-3$ \\
\hline PUFF2 & 0.24 & 0.1 & 10.09 & 0.07 & 0.04 & $6 . E-4$ & 3.E-3 & 4.E-3 & 0.03 \\
\hline \multicolumn{10}{|c|}{ RSUR2 (LATE): } \\
\hline PUFF1 & 1.0 & 0.06 & 0.03 & 0.09 & $3 E-3$ & $1 E-3$ & 4.E-4 & 4. $E-4$ & $3 E-3$ \\
\hline \multicolumn{10}{|c|}{ Modified Relense Fractions } \\
\hline \multicolumn{10}{|c|}{ MPB3 (MID): } \\
\hline PUFF1 & 0.76 & $1.8 \mathrm{E}-3$ & $8 E-4$ & $1.2 \mathrm{E}-3$ & 5.E-4 & 5.E-5 & 1.E-5 & 5.E-5 & $6 . E-4$ \\
\hline PUFF2 & 0.24 & 0.032 & 0.032 & 0.024 & 0.016 & 3.E-4 & $1.5 \mathrm{E}-3$ & 2.E-3 & 0.02 \\
\hline \multicolumn{10}{|c|}{ MPB2 (MID)": } \\
\hline PUFF1 & 0.76 & 5.7E-3 & $5.7 \mathrm{E}-3$ & $1.7 \mathrm{E}-3$ & 1.E-3 & $1.2 \mathrm{E}-3$ & 3.E-4 & 3.E-4 & $1.2 \mathrm{E}-3$ \\
\hline PUFF2 & 0.24 & 0.057 & 0.053 & 0.04 & 0.023 & 6.E-4 & 3.E-3 & 4.E-3 & 0.03 \\
\hline \multicolumn{10}{|c|}{ MSUR2 (LATE): } \\
\hline PUFF1 & 1.0 & 0.042 & 0.021 & 0.063 & $3 \mathrm{E}-3$ & 1E-3 & 4.E-4 & 4.E-4 & $3 \mathrm{E}-3$ \\
\hline
\end{tabular}


Table 4.3.2 Original and Modified Release Fractions (Continued)

\begin{tabular}{||l|c|c|c|c|c|c||}
\hline \multicolumn{7}{|c||}{ Additional Source Term Characteristics } \\
\hline Source Term & Purf & (Hr) & Heat (W) & Height (M) & Delay (H) & Duration (H) \\
\hline RPB3 & 1 & 4.8 & 0. & 30 & 4.8 & 2.5 \\
\hline & 2 & & 0. & 30. & 7.3 & 4 \\
\hline RPB2 & 1 & 4.8 & $6.4 E 7$ & 30. & 7.3 & 0.25 \\
\hline & 2 & & $2.3 E 6$ & 30. & 7.55 & 4 \\
\hline RSUR2 & 1 & 5 & $5.2 E 5$ & 10. & 12 & 3 \\
\hline
\end{tabular}

a. Timing class.

b. Puff 1 and Puff 2 indicate the total release occurs in two distinct phases.

Fractional releases are provided for each phase. 
Table 4.3.3 Release Inventories Compared to the Shutdown Core Inventory for the Two BWR Source Terms (Conservative Evacuation Case)

\begin{tabular}{|c|c|c|c|c|c|c|}
\hline Isotope & Group & $\begin{array}{c}\text { Core } \\
\text { Inventory } \\
\text { (Ci) }\end{array}$ & $\begin{array}{c}\text { MPB2 } \\
\text { (Ci) }\end{array}$ & $\begin{array}{c}\text { MPB2 } \\
\text { Fraction }\end{array}$ & $\begin{array}{c}\text { MPB3 } \\
\text { (Ci) }\end{array}$ & $\begin{array}{c}\text { MPB3 } \\
\text { Fraction }\end{array}$ \\
\hline CO-58 & 6 & $5.8 \mathrm{E}+05$ & $1.0 \mathrm{E}+03$ & $1.8 \mathrm{E}-03$ & $2.0 \mathrm{E}+02$ & 3.5E-04 \\
\hline CO-60 & 6 & $7.0 \mathrm{E}+05$ & $1.3 E+03$ & $1.8 \mathrm{E}-03$ & $2.4 E+02$ & 3.5E-04 \\
\hline KR-85 & 1 & $9.5 \mathrm{E}+05$ & $9.5 E+05$ & $1.0 \mathrm{E}+\infty 0$ & $9.5 E+05$ & $1.0 E+00$ \\
\hline KR-85M & 1 & $3.5 \mathrm{E}+07$ & $1.1 \mathrm{E}+07$ & 3.2E-01 & $1.5 \mathrm{E}+07$ & 4.4E-01 \\
\hline KR-87 & 1 & $6.3 E+07$ & $1.1 \mathrm{E}+06$ & $1.8 \mathrm{E}-02$ & $3.7 \mathrm{E}+06$ & $5.9 \mathrm{E}-02$ \\
\hline KR-88 & 1 & $8.5 E+07$ & $1.4 \mathrm{E}+07$ & $1.6 \mathrm{E}-01$ & $2.3 E+07$ & 2.7E-01 \\
\hline RB-86 & 3 & $5.3 \mathrm{E}+04$ & $3.1 \mathrm{E}+03$ & $5.8 \mathrm{E}-02$ & $2.2 E+03$ & 4.1E-02 \\
\hline SR-89 & 5 & $1.1 E+08$ & $2.5 E+06$ & $2.4 \mathrm{E}-02$ & $2.2 E+06$ & $2.0 \mathrm{E}-02$ \\
\hline SR-90 & 5 & $7.5 \mathrm{E}+06$ & $1.8 \mathrm{E}+05$ & 2.4E-02 & $1.5 \mathrm{E}+05$ & $2.0 \mathrm{E}-02$ \\
\hline SR-91 & 5 & $1.4 E+08$ & $1.9 E+06$ & $1.4 \mathrm{E}-02$ & $1.7 \mathrm{E}+06$ & $1.2 \mathrm{E}-02$ \\
\hline SR-92 & 5 & $1.4 \mathrm{E}+08$ & $5.0 \mathrm{E}+05$ & 3.5E-03 & $4.6 E+05$ & $3.2 \mathrm{E}-03$ \\
\hline Y-90 & 7 & $8.0 \mathrm{E}+06$ & $3.8 \mathrm{E}+04$ & 4.8E-03 & $2.3 E+04$ & $2.8 \mathrm{E}-03$ \\
\hline$Y-91$ & 7 & $1.3 E+08$ & 4.3E+05 & 3.4E-03 & $2.0 \mathrm{E}+05$ & $1.6 \mathrm{E}-03$ \\
\hline Y-92 & 7 & $1.4 \mathrm{E}+08$ & $1.0 \mathrm{E}+06$ & 7.2E-03 & 8.7E+05 & $6.1 \mathrm{E}-03$ \\
\hline Y-93 & 7 & $1.6 \mathrm{E}+08$ & $3.2 \mathrm{E}+05$ & $2.0 \mathrm{E}-03$ & $1.5 \mathrm{E}+05$ & $9.2 \mathrm{E}-04$ \\
\hline ZR-95 & 7 & $1.7 \mathrm{E}+08$ & $5.6 \mathrm{E}+05$ & 3.3E-03 & $2.5 \mathrm{E}+05$ & $1.5 \mathrm{E}-03$ \\
\hline ZR-97 & 7 & $1.7 E+08$ & $4.2 E+05$ & $2.4 \mathrm{E}-03$ & $1.9 E+05$ & $1.1 \mathrm{E}-03$ \\
\hline NB-95 & 7 & $1.6 \mathrm{E}+08$ & $5.3 E+05$ & 3.3E-03 & $2.4 \mathrm{E}+05$ & $1.5 \mathrm{E}-03$ \\
\hline MO-99 & 6 & $1.8 \mathrm{E}+08$ & $3.1 E+05$ & 1.7E-03 & $6.0 E+04$ & $3.3 \mathrm{E}-04$ \\
\hline TC-99M & 6 & $1.6 \mathrm{E}+08$ & $3.0 \mathrm{E}+05$ & $1.9 \mathrm{E}-03$ & $5.9 \mathrm{E}+04$ & 3.7E-04 \\
\hline RU-103 & 6 & $1.4 \mathrm{E}+08$ & $2.5 \mathrm{E}+05$ & $1.8 \mathrm{E}-03$ & $4.9 E+04$ & $3.5 \mathrm{E}-04$ \\
\hline RU-105 & 6 & $9.3 E+07$ & $5.3 E+04$ & 5.7E-04 & $1.1 E+04$ & $1.2 \mathrm{E}-04$ \\
\hline RU-106 & 6 & $3.8 \mathrm{E}+07$ & $6.9 E+04$ & $1.8 \mathrm{E}-03$ & $1.3 E+04$ & $3.5 \mathrm{E}-04$ \\
\hline RH-105 & 6 & $7.0 \mathrm{E}+07$ & $1.2 \mathrm{E}+05$ & 1.7E-03 & $2.4 \mathrm{E}+04$ & $3.4 \mathrm{E}-04$ \\
\hline SB-127 & 4 & $8.8 E+06$ & $3.5 \mathrm{E}+05$ & $3.9 \mathrm{E}-02$ & $2.6 \mathrm{E}+05$ & $3.0 \mathrm{E}-02$ \\
\hline SB-129 & 4 & $3.1 \mathrm{E}+07$ & $3.8 \mathrm{E}+05$ & 1.3E-02 & $3.1 \mathrm{E}+05$ & $1.0 \mathrm{E}-02$ \\
\hline TE-127 & 4 & $8.6 \mathrm{E}+06$ & $3.6 \mathrm{E}+05$ & 4.2E-02 & 2.7E+05 & 3.2E-02 \\
\hline TE-127M & 4 & $1.2 \mathrm{E}+06$ & $4.8 E+04$ & 4.2E-02 & $3.6 \mathrm{E}+04$ & 3.2E-02 \\
\hline TE-129 & 4 & $2.9 \mathrm{E}+07$ & $5.2 E+05$ & $1.8 \mathrm{E}-02$ & $4.1 E+05$ & $1.4 \mathrm{E}-02$ \\
\hline
\end{tabular}


Table 4.3.3 (Continued)

\begin{tabular}{|c|c|c|c|c|c|c|}
\hline Isotope & Group & $\begin{array}{c}\text { Core } \\
\text { Inventory } \\
\text { (Ci) }\end{array}$ & $\begin{array}{c}\text { MPB2 } \\
\text { (Ci) }\end{array}$ & $\begin{array}{c}\text { MPB2 } \\
\text { Fraction }\end{array}$ & $\begin{array}{c}\text { MPB3 } \\
\text { (Ci) }\end{array}$ & $\begin{array}{c}\text { MPB3 } \\
\text { Fraction }\end{array}$ \\
\hline TE-129M & 4 & $7.6 \mathrm{E}+06$ & $3.1 E+05$ & $4.1 E-02$ & $2.4 E+05$ & $3.1 E-02$ \\
\hline TE-131M & 4 & $1.5 E+07$ & $5.1 E+05$ & $3.5 \mathrm{E}-02$ & $3.9 E+05$ & 2.7E-02 \\
\hline TE-132 & 4 & $1.4 E+08$ & $5.5 E+06$ & $3.9 \mathrm{E}-02$ & $4.2 E+06$ & $3.0 \mathrm{E}-02$ \\
\hline$I-131$ & 2 & $9.8 E+07$ & $6.0 E+06$ & $6.1 E-02$ & 4. $1 E+06$ & 4.1E-02 \\
\hline $\mathrm{I}-132$ & 2 & $1.4 E+08$ & $6.0 \mathrm{E}+06$ & $4.2 E-02$ & $4.5 E+06$ & 3.1E-02 \\
\hline$I-133$ & 2 & $2.1 E+08$ & $1.0 \mathrm{E}+07$ & $4.9 \mathrm{E}-02$ & $6.9 E+06$ & $3.3 E-02$ \\
\hline I-134 & 2 & $2.3 E+08$ & $3.7 \mathrm{E}+04$ & $1.6 E-04$ & $4.0 E+04$ & $1.8 E-04$ \\
\hline $1-135$ & 2 & $1.9 E+08$ & $5.5 E+06$ & $2.8 \mathrm{E}-02$ & $3.9 E+06$ & $2.0 \mathrm{E}-02$ \\
\hline XE-133 & 1 & $2.1 E+08$ & $2.0 E+08$ & 9.6E-01 & $2.0 E+08$ & $9.7 E-01$ \\
\hline XE-135 & 1 & $4.9 E+07$ & $3.2 E+07$ & $6.4 \mathrm{E}-01$ & $3.5 E+07$ & $7.1 \mathrm{E}-01$ \\
\hline CS-134 & 3 & $1.6 \mathrm{E}+07$ & $9.4 E+05$ & 5.9E-02 & $6.6 E+05$ & 4.1E-02 \\
\hline CS-136 & 3 & $4.3 E+06$ & $2.5 E+05$ & $5.8 \mathrm{E}-02$ & $1.7 E+05$ & $4.0 \mathrm{E}-02$ \\
\hline CS-137 & 3 & $9.6 E+06$ & $5.7 E+05$ & 5.9E-02 & $3.9 E+05$ & 4.1E-02 \\
\hline BA-139 & 9 & $1.9 E+08$ & $1.4 E+05$ & $7.2 E-04$ & $1.1 \mathrm{E}+05$ & 5.7E-04 \\
\hline BA-140 & 9 & $1.9 E+08$ & $5.7 E+06$ & $3.1 \mathrm{E}-02$ & $3.8 E+06$ & $2.0 \mathrm{E}-02$ \\
\hline LA-140 & 7 & $1.9 E+08$ & $1.3 E+06$ & $6.6 \mathrm{E}-03$ & $7.0 \mathrm{E}+05$ & $3.7 E-03$ \\
\hline LA-141 & 7 & $1.8 E+08$ & $1.5 E+05$ & 8.8E-04 & $7.4 E+04$ & 4.2E-04 \\
\hline LA-142 & 7 & $1.7 E+08$ & $2.1 E+04$ & $1.2 \mathrm{E}-04$ & $1.1 \mathrm{E}+04$ & $6.4 E-05$ \\
\hline CE-141 & 8 & $1.7 E+08$ & $7.3 E+05$ & 4.3E-03 & $3.5 E+05$ & $2.0 \mathrm{E}-03$ \\
\hline CE-143 & 8 & $1.7 E+08$ & $6.1 E+05$ & $3.7 E-03$ & $2.9 E+05$ & $1.8 E-03$ \\
\hline CE-144 & 8 & $1.1 E+08$ & 4.7E+05 & 4.3E-03 & $2.3 E+05$ & $2.0 E-03$ \\
\hline PR-143 & 7 & $1.6 E+08$ & $5.4 E+05$ & $3.3 \mathrm{E}-03$ & $2.5 E+05$ & $1.5 E-03$ \\
\hline ND-147 & 7 & $7.2 \mathrm{E}+07$ & $2.3 E+05$ & $3.2 \mathrm{E}-03$ & $1.1 E+05$ & $1.5 E-03$ \\
\hline NP-239 & 8 & $2.2 E+09$ & $8.5 E+06$ & $3.9 E-03$ & $4.0 E+06$ & $1.9 E-03$ \\
\hline PU-238 & 8 & $1.5 E+05$ & $6.5 E+02$ & 4.3E-03 & $3.1 E+02$ & $2.1 E-03$ \\
\hline PU-239 & 8 & $3.8 \mathrm{E}+04$ & $1.6 E+02$ & 4.3E.03 & $7.8 E+01$ & $2.1 E-03$ \\
\hline PU-240 & 8 & $4.8 \mathrm{E}+04$ & $2.0 \mathrm{E}+02$ & 4.3E-03 & $9.8 \mathrm{E}+01$ & $2.0 \mathrm{E}-03$ \\
\hline PU-241 & 8 & $8.2 E+06$ & $3.5 E+04$ & 4.3E-03 & $1.7 E+04$ & $2.1 E-03$ \\
\hline AM-241 & 7 & $8.3 E+03$ & $2.8 E+01$ & $3.3 E-03$ & $1.3 \mathrm{E}+01$ & $1.5 E-03$ \\
\hline CM-242 & 7 & $2.2 E+06$ & $7.3 E+03$ & $3.3 E-03$ & $3.3 E+03$ & $1.5 \mathrm{E}-03$ \\
\hline CM-244 & 7 & $1.2 E+05$ & $3.9 E+02$ & $3.3 E-03$ & $1.8 \mathrm{E}+02$ & $1.5 E-03$ \\
\hline
\end{tabular}


Table 4.3.4 Release Inventory Compared to the Shutdown Core Inventory for the PWR Source Term (Conservative Evacuation Case)

\begin{tabular}{|c|c|c|c|c|}
\hline Isotope & Group & $\begin{array}{c}\text { Core } \\
\text { Inventory } \\
(\mathbf{C i})\end{array}$ & $\begin{array}{c}\text { MSUR2 } \\
\text { (Ci) }\end{array}$ & $\begin{array}{l}\text { MSUR2 } \\
\text { Fraction }\end{array}$ \\
\hline CO-58 & 6 & $9.7 E+05$ & $9.6 E+02$ & $9.9 \mathrm{E}-04$ \\
\hline$C O-60$ & 6 & $7.4 \mathrm{E}+05$ & $7.4 \mathrm{E}+02$ & $1.0 \mathrm{E}-03$ \\
\hline KR-85 & 1 & $7.5 \mathrm{E}+05$ & $7.5 E+05$ & $1.0 \mathrm{E}+00$ \\
\hline KR-85M & 1 & $3.5 \mathrm{E}+07$ & $5.5 E+06$ & $1.6 \mathrm{E}-01$ \\
\hline KR-87 & 1 & $6.4 \mathrm{E}+07$ & $9.0 \mathrm{E}+04$ & $1.4 \mathrm{E}-03$ \\
\hline KR-88 & 1 & $8.6 \mathrm{E}+07$ & $4.4 E+06$ & $5.1 \mathrm{E}-02$ \\
\hline RB-86 & 3 & $5.7 E+04$ & $1.7 E+03$ & $2.9 E-02$ \\
\hline SR-89 & 5. & $1.1 \mathrm{E}+08$ & $3.2 E+05$ & $3.0 \mathrm{E}-03$ \\
\hline SR-90 & 5 & $5.8 \mathrm{E}+06$ & $1.8 \mathrm{E}+04$ & $3.0 \mathrm{E}-03$ \\
\hline SR-91 & 5 & $1.4 E+08$ & $1.7 E+05$ & $1.2 \mathrm{E}-03$ \\
\hline SR-92 & 5 & $1.4 \mathrm{E}+08$ & $2.0 \mathrm{E}+04$ & $1.4 \mathrm{E}-04$ \\
\hline Y-90 & 7 & $6.3 E+06$ & 4.3E +03 & $6.9 \mathrm{E}-04$ \\
\hline$Y-91$ & 7 & $1.3 E+08$ & $5.4 \mathrm{E}+04$ & 4.1E-04 \\
\hline Y-92 & 7 & $1.5 E+08$ & $7.5 E+04$ & $5.2 \mathrm{E}-04$ \\
\hline Y-93 & 7 & $1.6 E+08$ & $2.9 E+04$ & $1.8 \mathrm{E}-04$ \\
\hline ZR-95 & 7 & $1.7 \mathrm{E}+08$ & $6.6 E+04$ & $4.0 \mathrm{E}-04$ \\
\hline ZR-97 & 7 & $1.7 E+08$ & $4.2 E+04$ & $2.4 \mathrm{E}-04$ \\
\hline NB-95 & 7 & $1.6 \mathrm{E}+08$ & $6.3 E+04$ & 4.0E-04 \\
\hline MO-99 & 6 & $1.8 \mathrm{E}+08$ & $1.6 \mathrm{E}+05$ & $8.8 \mathrm{E}-04$ \\
\hline TC-99M & 6 & $1.6 \mathrm{E}+08$ & $1.7 E+05$ & $1.1 \mathrm{E}-03$ \\
\hline RU-103 & 6 & $1.4 E+08$ & $1.4 \mathrm{E}+05$ & 9.9E-04 \\
\hline RU-105 & 6 & $8.9 E+07$ & $1.4 \mathrm{E}+04$ & $1.5 \mathrm{E}-04$ \\
\hline RU-106 & 6 & $3.1 \mathrm{E}+07$ & $3.1 E+04$ & $1.0 \mathrm{E}-03$ \\
\hline RH-105 & 6 & $6.2 \mathrm{E}+07$ & $5.7 \mathrm{E}+04$ & $9.2 \mathrm{E}-04$ \\
\hline SB-127 & 4 & $8.4 \mathrm{E}+06$ & $6.9 E+05$ & $8.2 \mathrm{E}-02$ \\
\hline SB-129 & 4 & $3.0 \mathrm{E}+07$ & $3.9 E+05$ & $1.3 \mathrm{E}-02$ \\
\hline TE-127 & 4 & $8.1 E+06$ & $7.2 \mathrm{E}+05$ & $8.9 \mathrm{E}-02$ \\
\hline TE-127M & 4 & $1.1 \mathrm{E}+06$ & $9.6 \mathrm{E}+04$ & 9.0E-02 \\
\hline TE-129 & 4 & $2.8 \mathrm{E}+07$ & $5.4 \mathrm{E}+05$ & $1.9 \mathrm{E}-02$ \\
\hline
\end{tabular}


Table 4.3.4 (Continued)

4 Results

\begin{tabular}{|c|c|c|c|c|}
\hline Isotope & Group & $\begin{array}{c}\text { Core } \\
\text { Inventory } \\
\text { (Ci) }\end{array}$ & $\begin{array}{c}\text { MSUR2 } \\
\text { (Ci) }\end{array}$ & $\begin{array}{l}\text { MSUR2 } \\
\text { Fraction }\end{array}$ \\
\hline TE-129M & 4 & $7.4 \mathrm{E}+06$ & $6.5 E+05$ & $8.9 E-02$ \\
\hline TE-131M & 4 & $1.4 \mathrm{E}+07$ & $9.6 \mathrm{E}+05$ & $6.8 \mathrm{E}-02$ \\
\hline TE-132 & 4 & $1.4 E+08$ & $1.1 \mathrm{E}+07$ & $8.1 E-02$ \\
\hline I-131 & 2 & $9.7 \mathrm{E}+07$ & $5.6 E+06$ & $5.8 \mathrm{E}-02$ \\
\hline I-132 & 2 & $1.4 E+08$ & $1.2 \mathrm{E}+07$ & $8.1 E-02$ \\
\hline I-133 & 2 & $2.0 \mathrm{E}+08$ & $8.2 E+06$ & 4.0E-02 \\
\hline I-134 & 2 & $2.2 \mathrm{E}+08$ & $1.0 \mathrm{E}+03$ & $4.5 E-06$ \\
\hline I-135 & 2 & $1.9 \mathrm{E}+08$ & $3.3 E+06$ & $1.7 \mathrm{E}-02$ \\
\hline XE-133 & 1 & $2.0 \mathrm{E}+08$ & $1.9 E+08$ & $9.4 \mathrm{E}-01$ \\
\hline XE-135 & 1 & $3.8 \mathrm{E}+07$ & $1.9 \mathrm{E}+07$ & $5.0 \mathrm{E}-01$ \\
\hline CS-134 & 3 & $1.3 \mathrm{E}+07$ & $3.9 E+05$ & $3.0 \mathrm{E}-02$ \\
\hline CS-136 & 3 & $4.0 \mathrm{E}+06$ & $1.2 E+05$ & $2.9 \mathrm{E}-02$ \\
\hline CS-137 & 3 & $7.3 E+06$ & $2.2 E+05$ & $3.0 \mathrm{E}-02$ \\
\hline BA-139 & 9 & $1.9 \mathrm{E}+08$ & $1.4 \mathrm{E}+03$ & $7.4 \mathrm{E}-06$ \\
\hline BA-140 & 9 & $1.9 \mathrm{E}+08$ & $5.5 \mathrm{E}+05$ & $2.9 \mathrm{E}-03$ \\
\hline LA-140 & 7 & $1.9 \mathrm{E}+08$ & $1.7 \mathrm{E}+05$ & 8.7E-04 \\
\hline LA-141 & 7 & $1.8 \mathrm{E}+08$ & $8.5 E+n 3$ & $4.8 \mathrm{E}-05$ \\
\hline LA-142 & 7 & $1.7 E+08$ & $3.6 \mathrm{E}+02$ & $2.1 \mathrm{E}-06$ \\
\hline CE-141 & 8 & $1.7 \mathrm{E}+08$ & $6.8 \mathrm{E}+04$ & 4.0E-04 \\
\hline CE-143 & 8 & $1.7 \mathrm{E}+08$ & $5.1 E+04$ & $3.1 E-04$ \\
\hline CE-144 & 8 & $1.0 \mathrm{E}+08$ & 4.1E +04 & 4.0E-04 \\
\hline PR-143 & 7 & $1.6 \mathrm{E}+08$ & $6.5 E+04$ & $4.0 \mathrm{E}-04$ \\
\hline ND-147 & 7 & $7.3 E+07$ & $2.8 \mathrm{E}+04$ & $3.9 \mathrm{E}-04$ \\
\hline NP-239 & 8 & $1.9 \mathrm{E}+09$ & $6.7 E+05$ & 3.5E-04 \\
\hline PU-238 & 8 & $1.1 \mathrm{E}+05$ & $4.4 E+01$ & 4.0E-04 \\
\hline PU-239 & 8 & $2.5 \mathrm{E}+04$ & $1.0 \mathrm{E}+01$ & 4.0E-04 \\
\hline PU-240 & 8 & $3.1 E+04$ & $1.3 E+01$ & 4.0E-04 \\
\hline PU-241 & 8 & $5.3 E+06$ & $2.1 E+03$ & 4.0E-04 \\
\hline AM-241 & 7 & $3.5 \mathrm{E}+03$ & $1.4 \mathrm{E}+00$ & 4.0E-04 \\
\hline CM-242 & 7 & $1.3 E+06$ & $5.3 E+02$ & 4.0E-04 \\
\hline CM-244 & 7 & $7.8 E+04$ & $3.1 E+01$ & 4.0E-04 \\
\hline
\end{tabular}


Table 4.3.5 Release Inventories Compared to the Shutdown Core Inventory (by Group) for the Two BWR Source Terms (Conservative Evacuation Case)

\begin{tabular}{|c|c|c|c|c|c||}
\hline Group & $\begin{array}{c}\text { Core Inventory } \\
(\mathrm{Ci})\end{array}$ & $\begin{array}{c}\text { MPB2 } \\
(\mathrm{Ci})\end{array}$ & $\begin{array}{c}\text { MPB2 } \\
\text { Fraction }\end{array}$ & $\begin{array}{c}\text { MPB3 } \\
(\mathrm{Ci})\end{array}$ & $\begin{array}{c}\text { MPB3 } \\
\text { Fraction }\end{array}$ \\
\hline 1 & $4.4 \mathrm{E}+08$ & $2.6 \mathrm{E}+08$ & $5.9 \mathrm{E}-01$ & $2.8 \mathrm{E}+08$ & $6.3 \mathrm{E}-01$ \\
\hline 2 & $8.7 \mathrm{E}+08$ & $2.8 \mathrm{E}+07$ & $3.2 \mathrm{E}-02$ & $1.9 \mathrm{E}+07$ & $2.2 \mathrm{E}-02$ \\
\hline 3 & $3.0 \mathrm{E}+07$ & $1.8 \mathrm{E}+06$ & $5.9 \mathrm{E}-02$ & $1.2 \mathrm{E}+06$ & $4.1 \mathrm{E}-02$ \\
\hline 4 & $2.4 \mathrm{E}+08$ & $8.0 \mathrm{E}+06$ & $3.3 \mathrm{E}-02$ & $6.1 \mathrm{E}+06$ & $2.5 \mathrm{E}-02$ \\
\hline 5 & $3.9 \mathrm{E}+08$ & $5.1 \mathrm{E}+06$ & $1.3 \mathrm{E}-02$ & $4.4 \mathrm{E}+06$ & $1.1 \mathrm{E}-02$ \\
\hline 6 & $6.9 \mathrm{E}+08$ & $1.1 \mathrm{E}+06$ & $1.6 \mathrm{E}-03$ & $2.2 \mathrm{E}+05$ & $3.2 \mathrm{E}-04$ \\
\hline 7 & $1.7 \mathrm{E}+09$ & $5.6 \mathrm{E}+06$ & $3.2 \mathrm{E}-03$ & $3.1 \mathrm{E}+06$ & $1.8 \mathrm{E}-03$ \\
\hline 8 & $2.6 \mathrm{E}+09$ & $1.0 \mathrm{E}+07$ & $3.9 \mathrm{E}-03$ & $4.9 \mathrm{E}+06$ & $1.9 \mathrm{E}-03$ \\
\hline 9 & $3.8 \mathrm{E}+08$ & $5.9 \mathrm{E}+06$ & $1.6 \mathrm{E}-02$ & $3.9 \mathrm{E}+06$ & $1.0 \mathrm{E}-02$ \\
\hline
\end{tabular}

Table 4.3.6 Release Inventory Compared to the Shutdown Core Inventory (by Group) for the PWR Source Term (Conservative Evacuation Case)

\begin{tabular}{|c|c|c|c||}
\hline Group & $\begin{array}{c}\text { Core Inventory } \\
(\mathrm{Ci})\end{array}$ & $\begin{array}{c}\text { MSUR2 } \\
(\mathrm{Ci})\end{array}$ & $\begin{array}{c}\text { MSUR2 } \\
\text { Fraction }\end{array}$ \\
\hline 1 & $4.3 \mathrm{E}+08$ & $2.2 \mathrm{E}+08$ & $5.2 \mathrm{E}-01$ \\
\hline 2 & $8.6 \mathrm{E}+08$ & $2.9 \mathrm{E}+07$ & $3.3 \mathrm{E}-02$ \\
\hline 3 & $2.4 \mathrm{E}+07$ & $7.2 \mathrm{E}+05$ & $3.0 \mathrm{E}-02$ \\
\hline 4 & $2.4 \mathrm{E}+08$ & $1.5 \mathrm{E}+07$ & $6.5 \mathrm{E}-02$ \\
\hline 5 & $4.0 \mathrm{E}+08$ & $5.3 \mathrm{E}+05$ & $1.3 \mathrm{E}-03$ \\
\hline 6 & $6.6 \mathrm{E}+08$ & $5.7 \mathrm{E}+05$ & $8.6 \mathrm{E}-04$ \\
\hline 7 & $1.7 \mathrm{E}+09$ & $6.0 \mathrm{E}+05$ & $3.5 \mathrm{E}-04$ \\
\hline 8 & $2.4 \mathrm{E}+09$ & $8.4 \mathrm{E}+05$ & $3.5 \mathrm{E}-04$ \\
\hline 9 & $3.8 \mathrm{E}+08$ & $5.5 \mathrm{E}+05$ & $1.5 \mathrm{E}-03$ \\
\hline
\end{tabular}


Table 4.3.7 Estimated Number of Individuals Exposed to Doses Exceeding Fatality Threshold Doses (Conservative Evacuation Case)

\begin{tabular}{|c|c|c|c|c|}
\hline Critical Organ & Source Term & $\begin{array}{c}\text { Evacuees } \\
(95 \%)^{b}\end{array}$ & $\begin{array}{c}\text { Non-Evacuees } \\
(5 \%)\end{array}$ & $\begin{array}{c}\text { Total }^{\mathbf{c}} \\
\text { (Weighted) }\end{array}$ \\
\hline \multirow[t]{3}{*}{ Red Marrow } & MPB2 & 9.2 & 57 & 11.6 \\
\hline & MPB3 & 16 & 47 & 17.4 \\
\hline & MSUR2 & 0 & 107 & 5.4 \\
\hline \multirow[t]{3}{*}{ Lungs } & MPB2 & 9.0 & 17 & 9.4 \\
\hline & MPB3 & 8.8 & 13 & 9.0 \\
\hline & MSUR2 & 0 & 27 & 1.4 \\
\hline \multirow[t]{3}{*}{ Lower Large Intestine } & MPB2 & 0.7 & 3 & 0.8 \\
\hline & MPB3 & 1.4 & 3 & 1.4 \\
\hline & MSUR2 & 0 & 6 & 0.3 \\
\hline
\end{tabular}

'The three critical organs in the early fatality health effects model the threshold doses for red marrow, lungs and the lower large intestine are 150,500 and 800 Rem, respectively.

The entry within the parenthesis indicates the percent of the total population that is assumed to evacuate.

Total represents the weighted sum: $95 \%$ of the evacuees plus $5 \%$ of the non-evacuees in this case. 
4 Results

Table 4.3.8 Estimated Number of Mean Early Fatalities from Four Adjusted Source Terms: Adjusted to Result in One Early Fatality

Emergency Response: Conservative Evacuation Case

Population Distribution: Uniform 10 People/Sq. Mi.

Exclusion Zone Boundary: 3 (0.4 mile)

80\% Weather with 3940 ft. Mixing Height

\begin{tabular}{|c|c|c|c|c|}
\hline & Evacuees & Non-Evacuees & Total & Timing Bin \\
\hline \multicolumn{5}{|c|}{$0-1.4$ miles } \\
\hline MPB1 & $7.83 \mathrm{E}-01 * .95=0.744$ & $3.32 \mathrm{E}+00 * .05=0.166$ & 0.909 & Late \\
\hline MPB6 & $9.22 \mathrm{E}-01 * .95=0.876$ & $3.71 \mathrm{E}+00 * .05=0.186$ & 1.06 & Late \\
\hline MSEQ1 & $1.24 \mathrm{E}+00 * .95=1.18$ & $2.54 \mathrm{E}+00 * .05=0.127$ & 1.30 & Mid \\
\hline MSEQ5 & $1.31 \mathrm{E}+00 * .95=1.24$ & $1.77 \mathrm{E}+00 * .05=0.089$ & 1.33 & Early \\
\hline \multicolumn{5}{|c|}{ Population Weighted Risk 0-1.4 Miles } \\
\hline MPB1 & $1.7 \mathrm{E}-2 * .95=1.3 \mathrm{E}-2$ & $5.9 \mathrm{E}-2 * .05=2.9 \mathrm{E}-3$ & $1: 6 \mathrm{E}-2$ & Late \\
\hline MPB6 & $1.7 \mathrm{E}-2 * .95=1.6 \mathrm{E}-2$ & $6.6 \mathrm{E}-2 * .05=3.3 \mathrm{E}-3$ & $1.9 \mathrm{E}-2$ & Late \\
\hline MSEQ1 & $2.2 \mathrm{E}-2 * .95=2.1 \mathrm{E}-2$ & $4.5 \mathrm{E}-2 * .05=2.2 \mathrm{E}-3$ & $2.3 \mathrm{E}-2$ & Mid \\
\hline MSEQ5 & $2.3 \mathrm{E}-2 * .95=2.2 \mathrm{E}-2$ & $3.1 \mathrm{E}-2 * .05=1.6 \mathrm{E}-3$ & $2.4 \mathrm{E}-2$ & Early \\
\hline
\end{tabular}

'MPB1 denotes the modified PB1 source term. 
Table 4.3.9 The Release Fractions for the Original Four Source Terms (Conservative Evacuation Case)

Population Distribution: Uniform 10 People/Sq. Mi.

Exclusion Zone Boundary: 3 ( 0.4 mile)

80\% Weather with 3940 ft. Mixing Height

\begin{tabular}{|c|c|c|c|c|c|c|c|c|c|c|}
\hline & $\mathrm{XE} / \mathrm{KR}$ & $I$ & CS & TE & $\mathbf{S R}$ & $\mathbf{R U}$ & LA & $\mathrm{CE}$ & $\overline{B A}$ & $\underset{\text { Bing }}{\text { Timing }}$ \\
\hline \multicolumn{10}{|c|}{ FOR RPB1 } & Late \\
\hline PUFF 1 & 0.76 & 0.01 & 0.01 & 3.E-3 & 1.E-3 & $1.2 \mathrm{E}-3$ & 3.E-4 & 3.E-4 & $1.2 \mathrm{E}-3$ & \\
\hline PUFF 2 & 0.24 & 0.1 & 0.09 & 0.07 & 0.04 & $6 . E-4$ & 3.E-3 & 4.E-3 & 0.03 & \\
\hline \multicolumn{10}{|c|}{ FOR RPB6 } & Late \\
\hline PUFF 1 & 0.76 & 0.04 & 0.04 & 0.012 & 4.E-3 & 5.E-3 & $1.2 \mathrm{E}-3$ & $1.2 \mathrm{E}-3$ & 5.E-3 & \\
\hline PUFF 2 & 0.24 & 0.4 & 0.36 & 0.28 & 0.16 & $2.4 \mathrm{E}-3$ & 0.01 & 0.02 & 0.12 & \\
\hline \multicolumn{10}{|c|}{ FOR RSEQ1 } & Mid \\
\hline PUFF 1 & 1.0 & 0.37 & 0.27 & 0.13 & 0.025 & 0.008 & $1.6 \mathrm{E}-3$ & 8.E-3 & 0.03 & \\
\hline PUFF 2 & 0.0 & 0.22 & 0.35 & 0.30 & 0.13 & 3.E-3 & 0.013 & 0.018 & 0.12 & \\
\hline \multicolumn{10}{|c|}{ FOR RSEQS } & Early \\
\hline PUFF 1 & 1.0 & 0.075 & 0.06 & 0.02 & 5.E-3 & 1.E-3 & 3.E-4 & 1.E-3 & 5.E-3 & \\
\hline PUFF 2 & 0.0 & 0.04 & 0.06 & 0.05 & 0.02 & $6 . E-4$ & 3.E-3 & 3.E-3 & 0.02 & \\
\hline \multicolumn{11}{|c|}{ The Release Fractions after Adjustment for MACCS Predicted One Early Fatality } \\
\hline \multicolumn{10}{|c|}{ FOR MPB1 } & Late \\
\hline PUFF 1 & 0.76 & 0.011 & 0.011 & 3.4E-3 & 1.E-3 & $1.2 \mathrm{E}-3$ & 3.E-4 & 3.E-4 & $1.2 \mathrm{E}-3$ & \\
\hline PUFF 2 & 0.24 & 0.13 & 0.12 & 0.09 & 0.04 & 6.E-4 & 3.E-3 & 4.E-3 & 0.03 & \\
\hline \multicolumn{10}{|c|}{ FOR MPB6 } & Late \\
\hline PUFF 1 & 0.76 & 0.04 & 0.04 & 0.012 & 4.E-3 & 5.E-3 & $1.2 \mathrm{E}-3$ & $1.2 \mathrm{E}-3$ & 5.E-3 & \\
\hline PUFF 2 & 0.24 & 0.39 & 0.35 & 0.27 & 0.16 & $2.4 \mathrm{E}-3$ & 0.01 & 0.02 & 0.12 & \\
\hline \multicolumn{10}{|c|}{ FOR MSEQ1 } & Mid \\
\hline PUFF 1 & 1.0 & 0.06 & 0.044 & 0.02 & 4.E-3 & 2.E-3 & 3.E-4 & 1.E-3 & 5.E-3 & \\
\hline PUFF 2 & 0.0 & 0.036 & 0.059 & 0.05 & 0.023 & 1.E-3 & 2.E-3 & 3.E-3 & 0.022 & \\
\hline \multicolumn{10}{|c|}{ FOR MSEQ5 } & Early \\
\hline PUFF 1 & 1.0 & 0.013 & 0.01 & 4.E-3 & 1.E-3 & 4.E-4 & 3.E-4 & 2.E-4 & 1.E-3 & \\
\hline PUFF 2 & 0.0 & 0.007 & 0.01 & 0.009 & 4.E-3 & 6.E-4 & 5.E-4 & 5.E-4 & 3.E-3 & \\
\hline
\end{tabular}


Table 4.3.10 Inventory Released Compared to the Shutdown Core Inventory for the Two BWR Source Terms (Conservative Evacuation and 10 People Per Square Mile)

\begin{tabular}{|c|c|c|c|c|c|c|}
\hline Isotope & Group & $\begin{array}{c}\text { Core } \\
\text { Inventory } \\
\text { (Ci) }\end{array}$ & MPB1 (Ci) & $\begin{array}{c}\text { MPB1 } \\
\text { Fraction }\end{array}$ & $\operatorname{MPB6}(\mathrm{Ci})$ & $\begin{array}{c}\text { MPB6 } \\
\text { Fraction }\end{array}$ \\
\hline Co-58 & 6 & $5.8 E+05$ & $1.0 \mathrm{E}+03$ & $1.8 \mathrm{E}-03$ & $2.0 \mathrm{E}+03$ & $3.5 \mathrm{E}-03$ \\
\hline$C 0-60$ & 6 & $7.0 \mathrm{E}+05$ & $1.3 E+03$ & $1.8 \mathrm{E}-03$ & $2.4 E+03$ & 3.5E-03 \\
\hline KR-85 & 1 & $9.5 \mathrm{E}+05$ & $9.5 \mathrm{E}+05$ & $1.0 \mathrm{E}+\infty 0$ & $4.5 E+05$ & 4.7E-01 \\
\hline KR-85M & 1 & $3.5 E+67$ & $5.8 E+06$ & 1.7E-01 & $2.7 \mathrm{E}+06$ & $7.9 \mathrm{E}-02$ \\
\hline KR-87 & 1 & $6.3 E+07$ & $1.1 \mathrm{E}+05$ & $1.8 \mathrm{E}-03$ & $5.3 E+04$ & 8.4E-04 \\
\hline KR-88 & 1 & $8.5 \mathrm{E}+07$ & $4.9 \mathrm{E}+06$ & 5.7E-02 & $2.3 \mathrm{E}+06$ & 2.7E-02 \\
\hline RB-86 & 3 & $5.3 E+04$ & $6.8 \mathrm{E}+03$ & 1.3E-01 & $9.6 \mathrm{E}+03$ & $1.8 \mathrm{E}-01$ \\
\hline SR-89 & 5 & $1.1 \mathrm{E}+08$ & $4.3 E+06$ & 4.1E-02 & $8.1 E+06$ & 7.7E-02 \\
\hline SR-90 & 5 & $7.5 E+06$ & $3.0 \mathrm{E}+05$ & 4.1E-02 & $5.8 E+05$ & $7.7 \mathrm{E}-02$ \\
\hline SR-91 & 5 & $1.4 E+08$ & $2.4 E+06$ & 1.7E-02 & $4.5 E+06$ & 3.3E-02 \\
\hline SR-92 & 5 & $1.4 E+08$ & $2.9 E+05$ & $2.0 \mathrm{E}-03$ & $5.5 E+05$ & 3.8E-03 \\
\hline$Y-90$ & 7 & $8.0 E+06$ & $6.0 \mathrm{E}+04$ & 7.5E-03 & $1.1 E+05$ & 1.3E-02 \\
\hline$Y-91$ & 7 & $1.3 E+08$ & 4.4E + 05 & 3.5E-03 & $7.2 \mathrm{E}+05$ & $5.6 \mathrm{E}-03$ \\
\hline Y -92 & 7 & $1.4 \mathrm{E}+08$ & $1.0 \mathrm{E}+06$ & 7.1E-03 & $1.9 E+06$ & $1.3 \mathrm{E}-02$ \\
\hline$Y-93$ & 7 & $1.6 \mathrm{E}+08$ & $2.4 E+05$ & $1.5 \mathrm{E}-03$ & $3.9 E+05$ & $2.4 \mathrm{E}-03$ \\
\hline ZR-95 & 7 & $1.7 \mathrm{E}+08$ & $5.6 \mathrm{E}+05$ & 3.3E-03 & $8.9 E+05$ & $5.2 \mathrm{E}-03$ \\
\hline ZR-97 & 7 & $1.7 E+08$ & $3.5 E+05$ & 2.0E-03 & $5.7 E+05$ & $3.3 E-03$ \\
\hline NB-95 & 7 & $1.6 E+08$ & $5.3 E+05$ & 3.3E-03 & $8.4 E+05$ & $5.3 E-03$ \\
\hline MO-99 & 6 & $1.8 \mathrm{E}+08$ & $2.9 \mathrm{E}+05$ & $1.6 \mathrm{E}-03$ & $5.7 \mathrm{E}+05$ & $3.1 \mathrm{E}-03$ \\
\hline TC-99M & 6 & $1.6 \mathrm{E}+08$ & $3.0 \mathrm{E}+05$ & $1.9 \mathrm{E}-03$ & $5.9 \mathrm{E}+05$ & 3.7E-03 \\
\hline RU-103 & 6 & $1.4 E+08$ & $2.5 E+05$ & $1.8 \mathrm{E}-03$ & $4.8 \mathrm{E}+05$ & $3.5 \mathrm{E}-03$ \\
\hline RU-105 & 6 & $9.3 \mathrm{E}+07$ & $2.8 \mathrm{E}+04$ & 2.9E-04 & $5.3 E+04$ & 5.7E-04 \\
\hline RU-106 & 6 & $3.8 E+07$ & $6.9 E+04$ & $1.8 \mathrm{E}-03$ & $1.3 \mathrm{E}+05$ & $3.5 \mathrm{E}-03$ \\
\hline RH-105 & 6 & $7.0 \mathrm{E}+07$ & $1.2 E+05$ & 1.7E-03 & $2.2 \mathrm{E}+05$ & $3.2 \mathrm{E}-03$ \\
\hline SB-127 & 4 & $8.8 E+06$ & $7.5 E+05$ & 8.5E-02 & $1.1 \mathrm{E}+06$ & $1.2 E-01$ \\
\hline SB-129 & 4 & $3.1 \mathrm{E}+07$ & $4.4 E+05$ & 1.4E-02 & $6.2 E+05$ & $2.0 \mathrm{E}-02$ \\
\hline TE-127 & 4 & $8.6 \mathrm{E}+06$ & $7.9 E+05$ & 9.3E-02 & $1.1 \mathrm{E}+06$ & $1.3 \mathrm{E}-01$ \\
\hline TE-127M & 4 & $1.2 \mathrm{E}+06$ & $1.1 E+05$ & 9.3E-02 & $1.5 E+05$ & 1.3E-01 \\
\hline TE-129 & 4 & $2.9 \mathrm{E}+07$ & $6.0 \mathrm{E}+05$ & 2.1E-02 & $8.5 E+05$ & $3.0 \mathrm{E}-02$ \\
\hline TE-129M & 4 & $7.6 \mathrm{E}+06$ & $7.0 \mathrm{E}+05$ & $9.2 \mathrm{E}-02$ & $9.9 \mathrm{E}+05$ & $1.3 E-01$ \\
\hline
\end{tabular}


Table 4.3.10 (Continued)

\begin{tabular}{|c|c|c|c|c|c|c|}
\hline Isotope & Group & $\begin{array}{c}\text { Core } \\
\text { Inventory } \\
\text { (Ci) }\end{array}$ & $\operatorname{MPB1}(\mathrm{Ci})$ & $\begin{array}{c}\text { MPBI } \\
\text { Fraction }\end{array}$ & MPB6 (Ci) & $\begin{array}{l}\text { MPB6 } \\
\text { Fraction }\end{array}$ \\
\hline TE-131M & 4 & $1.5 \mathrm{E}+07$ & $1.0 \mathrm{E}+06$ & $7.1 \mathrm{E}-02$ & $1.5 E+06$ & $1.0 E-01$ \\
\hline TE-132 & 4 & $1.4 \mathrm{E}+08$ & $1.2 \mathrm{E}+07$ & $8.4 E-02$ & $1.7 \mathrm{E}+07$ & $1.2 E-01$ \\
\hline $\mid-131$ & 2 & $9.8 E+07$ & $1.3 E+07$ & $1.4 \mathrm{E}-01$ & $1.9 E+07$ & $1.9 \mathrm{E}-01$ \\
\hline I-132 & 2 & $1.4 E+08$ & $1.2 \mathrm{E}+07$ & $8.7 E-02$ & $1.8 \mathrm{E}+07$ & $1.2 E-01$ \\
\hline I-133 & 2 & $2.1 E+08$ & $2.0 \mathrm{E}+07$ & 9.5E-02 & $2.8 E+07$ & $1.4 E-01$ \\
\hline I-134 & 2 & $2.3 E+08$ & $3.0 \mathrm{E}+03$ & $1.3 E-05$ & $4.3 E+03$ & $1.9 \mathrm{E}-05$ \\
\hline I-135 & 2 & $1.9 E+08$ & $8.0 \mathrm{E}+06$ & 4.1E-02 & $1.1 E+07$ & $5.9 E-02$ \\
\hline XE-133 & 1 & $2.1 \mathrm{E}+08$ & $2.0 \mathrm{E}+08$ & $9.5 \mathrm{E}-01$ & $9.3 E+07$ & $4.5 E-01$ \\
\hline XE-135 & 1 & $4.9 E+07$ & $2.9 E+07$ & $5.9 E-01$ & $2.2 \mathrm{E}+07$ & $4.4 E-01$ \\
\hline CS-134 & 3 & $1.6 \mathrm{E}+07$ & $2.1 E+06$ & $1.3 E-01$ & $2.9 E+06$ & $1.8 E-01$ \\
\hline CS-136 & 3 & $4.3 E+06$ & $5.5 \mathrm{E}+05$ & $1.3 \mathrm{E}-01$ & $7.7 E+05$ & $1.8 E-01$ \\
\hline CS-137 & 3 & $9.6 E+06$ & $1.3 E+06$ & $1.3 E-01$ & $1.8 E+06$ & $1.8 E-01$ \\
\hline BA-139 & 9 & $1.9 E+08$ & $1.7 E+04$ & 8.8E-05 & $3.1 E+04$ & $1.7 E-04$ \\
\hline$B A-140$ & 9 & $1.9 \mathrm{E}+08$ & $5.7 E+06$ & $3.0 \mathrm{E}-02$ & $1.1 E+07$ & $5.7 E-02$ \\
\hline LA-140 & 7 & $1.9 E+08$ & $1.6 E+06$ & $8.2 E-03$ & $2.8 E+06$ & $1.5 E-02$ \\
\hline LA-141 & 7 & $1.8 E+08$ & $7.4 E+04$ & $4.2 E-04$ & $1.2 \mathrm{E}+05$ & $6.7 E-04$ \\
\hline LA-142 & 7 & $1.7 \mathrm{E}+08$ & $3.4 E+03$ & $2.0 \mathrm{E}-05$ & $5.4 E+03$ & $3.2 \mathrm{E}-05$ \\
\hline CE-141 & 8 & $1.7 \mathrm{E}+08$ & $7.3 E+05$ & 4.3E-03 & $1.7 E+06$ & 9.9E-03 \\
\hline CE-143 & 8 & $1.7 E+08$ & $5.6 \mathrm{E}+05$ & $3.4 \mathrm{E}-03$ & $1.3 E+06$ & $7.8 \mathrm{E}-03$ \\
\hline CE-144 & 8 & $1.1 E+08$ & $4.7 E+05$ & $4.3 E-03$ & $1.1 E+06$ & $1.0 \mathrm{E}-02$ \\
\hline PR-143 & 7 & $1.6 \mathrm{E}+08$ & $5.4 E+05$ & $3.3 E-03$ & 8.7E+05 & $5.4 E-03$ \\
\hline ND-147 & 7 & $7.2 \mathrm{E}+07$ & $2.3 E+05$ & $3.2 \mathrm{E}-03$ & $3.7 E+05$ & 5.1E-03 \\
\hline NP-239 & 8 & $2.2 \mathrm{E}+09$ & $8.0 E+06$ & $3.7 \mathrm{E}-03$ & $1.9 E+07$ & 8.6E-03 \\
\hline PU-238 & 8 & $1.5 E+05$ & $6.5 E+02$ & 4.3E-03 & $1.5 E+03$ & $1.0 \mathrm{E}-02$ \\
\hline PU-239 & 8 & $3.8 E+04$ & $1.6 E+02$ & 4.3E-03 & $3.8 E+02$ & $1.0 \mathrm{E}-02$ \\
\hline PU-240 & 8 & $4.8 E+04$ & $2.0 E+02$ & 4.3E-03 & $4.8 E+02$ & $1.0 E-02$ \\
\hline PU-241 & 8 & $8.2 \mathrm{E}+06$ & $3.5 E+04$ & 4.3E-03 & $8.2 E+04$ & $1.0 \mathrm{E}-02$ \\
\hline AM-241 & 7 & $8.3 E+03$ & $2.8 \mathrm{E}+01$ & 3.3E-03 & $4.4 E+01$ & 5.3E-03 \\
\hline CM-242 & 7 & $2.2 E+06$ & $7.3 E+03$ & 3.3E-03 & $1.2 E+04$ & 5.3E-03 \\
\hline CM-244 & 7 & $1.2 E+05$ & $3.9 E+02$ & 3.3E-03 & $6.3 E+02$ & $5.3 E-03$ \\
\hline
\end{tabular}


Table 4.3.11 Inventory Released Compared to the Shutdown Core Inventory for the Two PWR Source Terms (Conservative Evacuation and 10 People Per Square Mile)

\begin{tabular}{|c|c|c|c|c|c|c|}
\hline Isotope & Group & $\begin{array}{l}\text { Core Inventory } \\
\text { (Ci) }\end{array}$ & MSEQ1 (Ci) & $\begin{array}{l}\text { MSEQ1 } \\
\text { Fraction }\end{array}$ & MSEQS (Ci) & $\begin{array}{l}\text { MSEQS } \\
\text { Fraction }\end{array}$ \\
\hline CO-58 & 6 & $9.7 E+05$ & $2.9 E+03$ & $3.0 \mathrm{E}-03$ & $9.7 E+02$ & $1.0 \mathrm{E}-03$ \\
\hline $00-60$ & 6 & $7.4 \mathrm{E}+05$ & $2.2 E+03$ & $3.0 \mathrm{E}-03$ & $7.4 E+02$ & $1.0 \mathrm{E}-03$ \\
\hline KR-85 & 1 & $7.5 E+05$ & $7.5 E+05$ & $1.0 \mathrm{E}+\infty 0$ & $7.5 E+05$ & $1.0 \mathrm{E}+\infty 0$ \\
\hline KR-85M & 1 & $3.5 \mathrm{E}+07$ & $1.5 E+07$ & $4.3 E-01$ & $3.0 \mathrm{E}+07$ & $8.6 \mathrm{E}-01$ \\
\hline KR-87 & 1 & $6.4 E+07$ & $3.1 E+06$ & 4.9E-02 & $3.7 E+07$ & $5.8 \mathrm{E}-01$ \\
\hline KR-88 & 1 & $8.6 E+07$ & $2.2 \mathrm{E}+07$ & $2.6 \mathrm{E}-01$ & $6.7 \mathrm{E}+07$ & $7.8 \mathrm{E}-01$ \\
\hline RB-86 & 3 & $5.7 E+04$ & $5.8 E+03$ & $1.0 \mathrm{E}-01$ & $1.1 E+03$ & 2.0E-02 \\
\hline SR-89 & 5 & $1.1 E+08$ & $2.9 \mathrm{E}+06$ & 2.7E-02 & $5.4 E+05$ & $5.0 \mathrm{E}-03$ \\
\hline SR-90 & 5 & $5.8 \mathrm{E}+06$ & $1.6 E+05$ & 2.7E-02 & $2.9 E+04$ & 5.0E-03 \\
\hline SR-91 & 5 & $1.4 E+08$ & $2.4 E+06$ & $1.8 \mathrm{E}-02$ & $6.3 E+05$ & 4.5E-03 \\
\hline SR-92 & 5 & $1.4 \mathrm{E}+08$ & $8.6 E+05$ & $5.9 E-03$ & $5.1 E+05$ & $3.5 E-03$ \\
\hline Y-90 & 7 & $6.3 E+06$ & $2.3 E+04$ & $3.7 E-03$ & $5.4 E+03$ & $8.6 E-04$ \\
\hline$X-91$ & 7 & $1.3 E+08$ & $3.1 \mathrm{E}+05$ & $2.4 \mathrm{E}-03$ & $1.1 \mathrm{E}+05$ & 8.0E-04 \\
\hline$Y-92$ & 7 & $1.5 E+08$ & $1.3 E+06$ & $8.9 E-03$ & $2.3 E+05$ & $1.6 E-03$ \\
\hline$Y-93$ & 7 & $1.6 E+08$ & $2.5 E+05$ & $1.5 E-03$ & $1.2 \mathrm{E}+05$ & $7.3 \mathrm{E}-04$ \\
\hline ZR-95 & 7 & $1.7 E+08$ & $3.8 E+05$ & 2.3E-03 & $1.3 E+05$ & 8.0E-04 \\
\hline ZR-97 & 7 & $1.7 \mathrm{E}+08$ & $3.1 E+05$ & $1.8 \mathrm{E}-03$ & $1.3 E+05$ & $7.6 \mathrm{E}-04$ \\
\hline NB-95 & 7 & $1.6 \mathrm{E}+08$ & $3.6 E+05$ & 2.3E-03 & $1.3 \mathrm{E}+05$ & 8.0E-04 \\
\hline MO-99 & 6 & $1.8 E+08$ & $5.2 \mathrm{E}+05$ & $2.8 E-03$ & $1.8 E+05$ & 9.9E-04 \\
\hline TC-99M & 6 & $1.6 E+08$ & $5.0 \mathrm{E}+05$ & $3.2 \mathrm{E}-03$ & $1.6 \mathrm{E}+05$ & $1.0 \mathrm{E}-03$ \\
\hline RU-103 & 6 & $1.4 \mathrm{E}+08$ & $4.1 E+05$ & $3.0 \mathrm{E}-03$ & $1.4 E+05$ & $1.0 \mathrm{E}-03$ \\
\hline RU-105 & 6 & $8.9 E+07$ & $1.1 \mathrm{E}+05$ & $1.2 \mathrm{E}-03$ & $7.3 E+04$ & 8.2E-04 \\
\hline RU-106 & 6 & $3.1 E+07$ & $9.3 E+04$ & $3.0 \mathrm{E}-03$ & $3.1 E+04$ & $1.0 \mathrm{E}-03$ \\
\hline RH-105 & 6 & $6.2 \mathrm{E}+07$ & $1.8 \mathrm{E}+05$ & $3.0 \mathrm{E}-03$ & $6.2 \mathrm{E}+04$ & $1.0 \mathrm{E}-03$ \\
\hline SB-127 & 4 & $8.4 E+06$ & $5.6 \mathrm{E}+05$ & 6.7E-02 & $1.1 E+05$ & $1.3 E-02$ \\
\hline SB-129 & 4 & $3.0 \mathrm{E}+07$ & $8.2 E+05$ & 2.7E-02 & $3.1 E+05$ & $1.0 \mathrm{E}-02$ \\
\hline TE-127 & 4 & $8.1 E+06$ & $5.7 E+05$ & $7.0 \mathrm{E}-02$ & $1.1 E+05$ & $1.3 \mathrm{E}-02$ \\
\hline TE-127M & 4 & $1.1 \mathrm{E}+06$ & $7.5 E+04$ & $7.0 \mathrm{E}-02$ & $1.4 \mathrm{E}+04$ & $1.3 \mathrm{E}-02$ \\
\hline TE-129 & 4 & $2.8 \mathrm{E}+07$ & $1.1 \mathrm{E}+06$ & $3.9 \mathrm{E}-02$ & $3.5 \mathrm{E}+05$ & $1.3 \mathrm{E}-02$ \\
\hline TE-129M & 4 & $7.4 E+06$ & $5.1 E+05$ & $7.0 \mathrm{E}-02$ & $9.6 E+04$ & $1.3 \mathrm{E}-02$ \\
\hline
\end{tabular}


Table 4.3.11 (Continued)

4 Results

\begin{tabular}{|c|c|c|c|c|c|c|}
\hline Isotope & Group & $\begin{array}{l}\text { Core Inventory } \\
\text { (Ci) }\end{array}$ & MSEQ1 (Ci) & $\begin{array}{l}\text { MSEQ1 } \\
\text { Fraction }\end{array}$ & MSEQS (Ci) & $\begin{array}{l}\text { MSEQS } \\
\text { Fraction }\end{array}$ \\
\hline TE-131M & 4 & $1.4 E+07$ & $8.6 E+05$ & $6.1 E-02$ & $1.8 \mathrm{E}+05$ & $1.3 \mathrm{E}-02$ \\
\hline TE-132 & 4 & $1.4 E+08$ & $9.3 E+06$ & $6.6 \mathrm{E}-02$ & $1.8 \mathrm{E}+06$ & $1.3 E-02$ \\
\hline $1-131$ & 2 & 9.7E+07 & $9.1 E+06$ & $9.4 \mathrm{E}-02$ & $1.9 \mathrm{E}+06$ & $2.0 \mathrm{E}-02$ \\
\hline $1-132$ & 2 & $1.4 E+08$ & $1.0 E+07$ & $7.3 \mathrm{E}-02$ & $2.6 E+06$ & $1.8 \mathrm{E}-02$ \\
\hline $1-133$ & 2 & $2.0 \mathrm{E}+08$ & $1.6 \mathrm{E}+07$ & $7.9 \mathrm{E}-02$ & $3.9 E+06$ & $1.9 \mathrm{E}-02$ \\
\hline $1-134$ & 2 & $2.2 E+08$ & $2.4 E+05$ & $1.1 \mathrm{E}-03$ & $1.8 \mathrm{E}+06$ & $8.0 E-03$ \\
\hline $1-135$ & 2 & $1.9 \mathrm{E}+08$ & $1.0 \mathrm{E}+07$ & $5.3 E-02$ & $3.4 E+06$ & $1.8 E-02$ \\
\hline XE-133 & 1 & $2.0 \mathrm{E}+08$ & $2.0 \mathrm{E}+08$ & 9.7E-01 & $2.0 \mathrm{E}+08$ & $9.9 E-01$ \\
\hline XE-135 & 1 & $3.8 E+07$ & $3.0 \mathrm{E}+07$ & $7.8 \mathrm{E}-01$ & $3.6 E+07$ & 9.3E-01 \\
\hline CS-134 & 3 & $1.3 \mathrm{E}+07$ & $1.3 E+06$ & $1.0 \mathrm{E}-01$ & $2.6 E+05$ & $2.0 \mathrm{E}-02$ \\
\hline CS-136 & 3 & $4.0 \mathrm{E}+06$ & $4.0 \mathrm{E}+05$ & $1.0 \mathrm{E}-01$ & $7.9 \mathrm{E}+04$ & 2.0E-02 \\
\hline CS-137 & 3 & $7.3 E+06$ & $7.5 E+05$ & $1.0 \mathrm{E}-01$ & $1.5 E+05$ & $2.0 \mathrm{E}-02$ \\
\hline BA-139 & 9 & $1.9 E+08$ & 2.7E+05 & $1.4 \mathrm{E}-03$ & $3.8 \mathrm{E}+05$ & $2.0 E-03$ \\
\hline BA-140 & 9 & $1.9 E+08$ & $5.0 \mathrm{E}+06$ & 2.7E-02 & $7.5 E+05$ & $4.0 \mathrm{E}-03$ \\
\hline LA-140 & 7 & $1.9 E+08$ & $8.8 E+05$ & $4.6 \mathrm{E}-03$ & $1.7 E+05$ & 8.7E-04 \\
\hline LA-141 & 7 & $1.8 \mathrm{E}+08$ & $1.4 E+05$ & 8.1E-04 & $1.1 E+05$ & $6.3 E-04$ \\
\hline LA-142 & 7 & $1.7 E+08$ & $2.9 E+04$ & $1.7 E-04$ & $7.7 E+04$ & 4.5E-04 \\
\hline CE-141 & 8 & $1.7 E+08$ & $6.8 E+05$ & 4.0E-03 & $1.2 E+05$ & $7.0 \mathrm{E}-04$ \\
\hline CE-143 & 8 & $1.7 E+08$ & $5.9 E+05$ & 3.5E-03 & $1.1 E+05$ & $6.8 E-04$ \\
\hline CE-144 & 8 & $1.0 \mathrm{E}+08$ & $4.1 E+05$ & $4.0 \mathrm{E}-03$ & $1.2 \mathrm{E}+04$ & $7.0 \mathrm{E}-04$ \\
\hline PR-143 & 7 & $1.6 E+08$ & $3.8 E+05$ & 2.3E-03 & $1.3 E+05$ & 8.0E-04 \\
\hline ND-147 & 7 & $7.3 E+07$ & $1.6 \mathrm{E}+05$ & 2.3E-03 & $5.8 \mathrm{E}+04$ & 8.0E-04 \\
\hline NP-239 & 8 & $1.9 \mathrm{E}+09$ & $7.3 \mathrm{E}+06$ & $3.7 E-03$ & $1.3 E+06$ & $6.9 \mathrm{E}-04$ \\
\hline PU-238 & 8 & $1.1 \mathrm{E}+05$ & $4.4 E+02$ & $4.0 E-03$ & $7.7 \mathrm{E}+01$ & $7.0 \mathrm{E}-04$ \\
\hline PU-239 & 8 & $2.5 E+04$ & $1.0 \mathrm{E}+02$ & $4.0 \mathrm{E}-03$ & $1.7 E+01$ & $7.0 \mathrm{E}-04$ \\
\hline PU-240 & 8 & $3.1 E+04$ & $1.3 E+02$ & 4.0E-03 & $2.2 \mathrm{E}+01$ & $7.0 \mathrm{E}-04$ \\
\hline PU-241 & 8 & $5.3 \mathrm{E}+06$ & $2.1 E+04$ & $4.0 \mathrm{E}-03$ & $3.7 E+03$ & $7.0 \mathrm{E}-04$ \\
\hline AM-241 & 7 & $3.5 E+03$ & $8.1 E+\infty 0$ & 2.3E-03 & $2.8 \mathrm{E}+00$ & 8.0E-04 \\
\hline CM-242 & 7 & $1.3 \mathrm{E}+06$ & $3.1 E+03$ & $2.3 E-03$ & $1.1 \mathrm{E}+03$ & 8.0E-04 \\
\hline CM-244 & 7 & $7.8 E+04$ & $1.8 E+02$ & 2.3E-03 & $6.3 E+01$ & 8.0E-04 \\
\hline
\end{tabular}


Table 4.3.12 Grouped Inventory Released Compared to the Shutdown Core Inventory for the Two BWR Source Terms (Conservative Evacuation and 10 People Per Square Mile)

\begin{tabular}{|c|c|c|c|c|c|}
\hline Group & $\begin{array}{c}\text { Core } \\
\text { Inventory } \\
(\mathrm{Ci})\end{array}$ & $\begin{array}{c}\text { MPB1 } \\
(\mathrm{Ci})\end{array}$ & $\begin{array}{c}\text { MPB1 } \\
\text { Fraction }\end{array}$ & $\begin{array}{c}\text { MPB6 } \\
(\mathrm{Ci})\end{array}$ & $\begin{array}{c}\text { MPB6 } \\
\text { Fraction }\end{array}$ \\
\hline 1 & $4.4 \mathrm{E}+08$ & $2.4 \mathrm{E}+06$ & $5.4 \mathrm{E}-01$ & $1.2 \mathrm{E}+06$ & $2.7 \mathrm{E}-01$ \\
\hline 2 & $8.7 \mathrm{E}+06$ & $5.3 \mathrm{E}+06$ & $6.2 \mathrm{E}-02$ & $7.6 \mathrm{E}+06$ & $8.8 \mathrm{E}-02$ \\
\hline 3 & $3.0 \mathrm{E}+06$ & $3.9 \mathrm{E}+06$ & $1.3 \mathrm{E}-01$ & $5.5 \mathrm{E}+06$ & $1.8 \mathrm{E}-01$ \\
\hline 4 & $2.4 \mathrm{E}+06$ & $1.6 \mathrm{E}+06$ & $6.8 \mathrm{E}-02$ & $2.3 \mathrm{E}+06$ & $9.6 \mathrm{E}-02$ \\
\hline 5 & $7.5 \mathrm{E}+06$ & $3.0 \mathrm{E}+05$ & $4.1 \mathrm{E}-02$ & $5.8 \mathrm{E}+05$ & $7.7 \mathrm{E}-02$ \\
\hline 6 & $6.9 \mathrm{E}+06$ & $1.1 \mathrm{E}+06$ & $1.5 \mathrm{E}-03$ & $2.1 \mathrm{E}+06$ & $3.0 \mathrm{E}-03$ \\
\hline 7 & $1.7 \mathrm{E}+06$ & $5.6 \mathrm{E}+06$ & $3.3 \mathrm{E}-03$ & $9.6 \mathrm{E}+06$ & $5.6 \mathrm{E}-03$ \\
\hline 8 & $2.6 \mathrm{E}+06$ & $9.8 \mathrm{E}+06$ & $3.8 \mathrm{E}-03$ & $2.3 \mathrm{E}+06$ & $8.7 \mathrm{E}-03$ \\
\hline 9 & $3.8 \mathrm{E}+08$ & $5.7 \mathrm{E}+06$ & $1.5 \mathrm{E}-02$ & $1.1 \mathrm{E}+06$ & $2.9 \mathrm{E}-02$ \\
\hline
\end{tabular}

Table 4.3.13 Grouped Inventory Released Compared to the Shutdown Core Inventory for the Two PWR Source Terms (Conservative Evacuation and 10 People Per Square Mile)

\begin{tabular}{|c|c|c|c|c|c||}
\hline Group & $\begin{array}{c}\text { Core Inrentory } \\
(\mathrm{Ci})\end{array}$ & $\begin{array}{c}\text { MSEQ1 } \\
(\mathrm{Ci})\end{array}$ & $\begin{array}{c}\text { MSEQ1 } \\
\text { Fraction }\end{array}$ & $\begin{array}{c}\text { MSEQS } \\
(\mathrm{Ci})\end{array}$ & $\begin{array}{c}\text { MSEQ5 } \\
\text { Fraction }\end{array}$ \\
\hline 1 & $4.3 \mathrm{E}+08$ & $2.7 \mathrm{E}+08$ & $6.3 \mathrm{E}-01$ & $3.7 \mathrm{E}+08$ & $8.7 \mathrm{E}-01$ \\
\hline 2 & $8.6 \mathrm{E}+08$ & $4.6 \mathrm{E}+07$ & $5.4 \mathrm{E}-02$ & $1.4 \mathrm{E}+07$ & $1.6 \mathrm{E}-02$ \\
\hline 3 & $2.4 \mathrm{E}+07$ & $2.5 \mathrm{E}+06$ & $1.0 \mathrm{E}-01$ & $4.9 \mathrm{E}+05$ & $2.0 \mathrm{E}-02$ \\
\hline 4 & $2.4 \mathrm{E}+08$ & $1.4 \mathrm{E}+07$ & $5.8 \mathrm{E}-02$ & $3.0 \mathrm{E}+06$ & $1.3 \mathrm{E}-02$ \\
\hline 5 & $5.8 \mathrm{E}+06$ & $1.6 \mathrm{E}+05$ & $2.7 \mathrm{E}-02$ & $2.9 \mathrm{E}+04$ & $5.0 \mathrm{E}-03$ \\
\hline 6 & $6.6 \mathrm{E}+08$ & $1.8 \mathrm{E}+06$ & $2.8 \mathrm{E}-03$ & $6.5 \mathrm{E}+05$ & $9.8 \mathrm{E}-04$ \\
\hline 7 & $1.7 \mathrm{E}+09$ & $4.5 \mathrm{E}+06$ & $2.6 \mathrm{E}-03$ & $1.4 \mathrm{E}+06$ & $8.2 \mathrm{E}-04$ \\
\hline 8 & $2.4 \mathrm{E}+09$ & $8.9 \mathrm{E}+06$ & $3.7 \mathrm{E}-03$ & $1.6 \mathrm{E}+06$ & $6.9 \mathrm{E}-04$ \\
\hline 9 & $3.8 \mathrm{E}+08$ & $5.2 \mathrm{E}+06$ & $1.4 \mathrm{E}-02$ & $1.1 \mathrm{E}+06$ & $3.0 \mathrm{E}-03$ \\
\hline
\end{tabular}




\subsection{Scaling Source Terms for the Sheltering Case}

In the sheltering case, 95 percent of the population in the emergency zone is assumed to take shelter for a period of 12 hours (following which the shelterees are instantly relocated, i.e., removed from the calculation); 5 percent of the population is assuzied to follow normal activity as in the conservative evacuation case. The reduction in dose and dose rate to those taking shelter are shown in Table 2.1.2.

Throe source terms were selected for modification, RPB2, RPB3, and RSUR2, (the same ones as in the Conservative evacuation case due to similarity of results). In this case, the radionuclide release fractions (mainly of the iodine, tellurium, and cesium groups) for each source term were scaled so as to produce a MACCS estimated value of one mean early fatality.

The results for the early fatalities for each modified source term, broken down by protective action assumption, i.e., the percentage of the population assumed to take shelter or follow normal activity, are shown in Table 4.4.1. Table 4.4.2 shows the characteristics in terms of the release fractions and timing of release of the original source terms RPB2, RPB3 and RSUR2 and the modified source terms MPB2, MPB3, and MSUR2 (after scaling).

Table 4.4.1 also shows the relative and total contributions to the estimated number of early fatalities from the populations under each protective action assumption for each modified source term. For MPB3, which is an early release, the adjusted release fractions for iodine ( $2.6 \%$ for both puffs) and tellurium $(2 \%)$ are such as to produce a relative contribution of an extimated 2.7 early fatalities from the population following normal activity and an estimated 0.86 early fatalities from sheltering population. Multiplying the relative contributions by the assumed fractions of the population participating in each protective action, we see that for MPB3, 82 percent of the early fatality weight comes from the shelterees and the remainder from those engaged in normal activity. For MPB2, the corresponding weight estimates are 70 percent and 30 percent. In the case of MSUR2, which is a very late release, there is a much larger relative contribution of an estimated 10.7 early fatalities from the population following normal activity as compared with an estimated 0.51 fatalities from sheltering population. This leads to more than half of the MACCS estimated early fatalities coming from the normal activity population.

Tables 4.4.3 and 4.4.4 show the inventories of each radionuclide released to the environment to produce the MACCS estimated one mean early fatality for the modified BWR (MPB2, MPB3) and PWR (MSUR2) source terms respectively. Tables 4.4.5 and 4.4.6 show the released inventories grouped by the standard isotope grouping scheme, for the modified BWR and PWR source terms, respectively.

Table 4.4.7 shows the total number of exposed individuals whose organ dose exceeds the threshold to the three organs (red marrow, lungs, lower large intestine) used in the MACCS early fatality health effects model. The sheltering assumption means that the population taking shelter stays in the area for 12 hours. Thus, there is always some exposure (even if at a reduced rate) to the shelterees. The number of people participating in each protective action assumption exceeding the early fatality threshold organ dose follows the same pattern observed in Table 4.4.1. For the MPB2 and MPB2 source terms, the major contribution to the total comes from the sheltered population while for MSUR2, it comes from the population following normal activity.

Tables B.4.1-B.4.3 of Appendix B provide the centerline dose to red marrow, lungs, and lower large intestine as a function of distance and for the different pathways that make up the acute (seven day) exposure. This data refers to the MPB3 source term only. As with the conservative evacuation assumption, this data illustrates that the dose to the sheltered population (95\% of the total) is almost as large as the dose to those following normal activity (5\% 


\section{Results}

of the total). These tables are condensed into Tables B.4.4-B.4.6 which show the relative percentage of dose at each distance for each pathway. These are divided by the total population, the sheltered population, and the normalactivity population. As expected, the red marrow dose is the dominant contributor. This is in contrast to the pathways for the conservative evacuation where cloudshine and inhalation were the main pathways, with inhalation being dominant. Again, as with the conservative evacuation case, doses to the lung and lower large intestine are dominated by the inhalation pathways in both groups. Cloudshine was the second largest contributor to lung and lower large intestine dose to the shelterees beyond about 0.5 miles and groundshine was the second largest contributor for the normal-activity population. 
Table 4.4.1 Estimated Number of Mean Early Fatalities from the Three Modified Source Terms (Sheltering Case)

Population Distribution: Uniform 1000 People/Sq. Mi.

Exclusion Zone Boundary: 0.4 mile

1 Hour Delay, 12 Hour Sheltering, Followed by Immediate Relocation for 95\% of Population.

The Release Fractions Were Adjusted to Result in One Early Fatality. 80\% Weather, 3940 Ft. Mixing Height

\begin{tabular}{||l|c|c|c|l||}
\hline \multicolumn{1}{|c|}{$\begin{array}{c}\text { Source } \\
\text { Term }\end{array}$} & $\begin{array}{c}\text { Shelter } \\
\text { Population } \\
(95 \%)\end{array}$ & $\begin{array}{c}\text { Normal Activity } \\
\text { Population } \\
(5 \%)\end{array}$ & $\begin{array}{c}\text { Weighted } \\
\text { Total }\end{array}$ & $\begin{array}{c}\text { Release Timing } \\
\text { Category }\end{array}$ \\
\hline MPB3 & 0.859 & 2.67 & 0.950 & Early \\
\hline MPB2 & 0.733 & 4.80 & 0.937 & Mid \\
\hline MSUR2 & 0.510 & 10.4 & 1.01 & Late \\
\hline
\end{tabular}


4 Results

Table 4.4.2 Adjustment of the Release Fractions to Result in One Early Fatality (Sheltering Case)

Population Distribution: Uniform 1000 People/Sq. Mi.

Exclusion Zone Boundary: 3 (0.4 mile)

80\% Weather with $3940 \mathrm{ft}$. Mixing Height

The Original Release Fractions for the Three Source Terms

\begin{tabular}{|c|c|c|c|c|c|c|c|c|c|c|c|c|}
\hline \multicolumn{13}{|c|}{ The Original Release Fractions for the Three Source Terms } \\
\hline & \multicolumn{2}{|c|}{$\mathbf{X E} / \mathbf{K R}$} & & 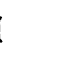 & CS & TE & \multicolumn{2}{|c|}{ SR } & RU & $\mathbf{L A}$ & CE & BA \\
\hline \multicolumn{13}{|c|}{ RPB3 (EARLY): } \\
\hline PUFF1 & \multicolumn{2}{|c|}{0.76} & \multicolumn{2}{|c|}{ 2.3E-3 } & 0.001 & $1.5 \mathrm{E}-3$ & \multicolumn{2}{|c|}{$5 \mathrm{E}-4$} & $5 E-5$ & 1E-5 & $5 E-5$ & $6 \mathrm{E}-4$ \\
\hline PUFF2 & \multicolumn{2}{|c|}{0.24} & \multicolumn{2}{|c|}{0.04} & 0.04 & 0.03 & \multicolumn{2}{|c|}{0.02} & $3 E-4$ & $1.5 \mathrm{E}-3$ & $2 E-3$ & 0.02 \\
\hline \multicolumn{13}{|c|}{ RPB2 (Mm): } \\
\hline PUFF1 & \multicolumn{2}{|c|}{0.76} & \multicolumn{2}{|c|}{0.01} & 0.01 & $3 E-3$ & \multicolumn{2}{|c|}{$1 \mathrm{E}-3$} & $1.2 \mathrm{E}-3$ & $3 E-4$ & $3 E-4$ & $1.2 \mathrm{E}-3$ \\
\hline PUFF2 & \multicolumn{2}{|c|}{0.24} & \multicolumn{2}{|c|}{0.1} & 0.09 & 0.07 & \multicolumn{2}{|c|}{0.04} & $6 E-4$ & $3 E-3$ & $4 E-3$ & 0.03 \\
\hline \multicolumn{13}{|c|}{ RSUR2 (LATE): } \\
\hline PUFF1 & \multicolumn{2}{|c|}{1.0} & \multicolumn{2}{|c|}{0.06} & 0.03 & 0.09 & \multicolumn{2}{|c|}{0.003} & 0.001 & $4 \mathrm{E}-4$ & $4 E-4$ & 0.003 \\
\hline \multicolumn{13}{|c|}{ The Release Fractions Adjusted to Produce One Early Fatality } \\
\hline \multicolumn{13}{|c|}{ MPB3 (EARLY): } \\
\hline PUFF1 & \multicolumn{2}{|c|}{0.76} & \multicolumn{2}{|c|}{$1.4 \mathrm{E}-3$} & $6 \mathrm{E}-4$ & $1 E-3$ & \multicolumn{2}{|c|}{$5 E-4$} & $5 E-5$ & IE-5 & $5 E-5$ & $6 E-4$ \\
\hline PUFF2 & \multicolumn{2}{|c|}{0.24} & \multicolumn{2}{|c|}{0.025} & 0.025 & 0.019 & \multicolumn{2}{|c|}{0.02} & $3 . E-4$ & $1.5 \mathrm{E}-3$ & $2 \mathrm{E}-3$ & 0.02 \\
\hline \multicolumn{13}{|c|}{ MPB2 (MID): } \\
\hline PUFF1 & & & & E-3 & $4.5 \mathrm{E}-3$ & $1.4 \mathrm{E}-3$ & & & $1.2 \mathrm{E}-3$ & $3 E-4$ & $3 E-4$ & $1.2 \mathrm{E}-3$ \\
\hline PUFF2 & & & & 45 & 0.04 & 0.03 & & & $6 E-4$ & $3 E-3$ & $4 E-3$ & 0.03 \\
\hline MSUR2 & & & & & & & & & & & & \\
\hline PUFF1 & & & & 03 & 0.015 & 0.045 & & & 0.001 & $4 E-4$ & $4 E-4$ & 0.003 \\
\hline & & & & & & racteristics & of $\mathrm{E}$ & ch & & & & \\
\hline Source & & $\mathbf{P}$ & & Ala & n (Hr) & Energy ( & & & ht (M) & Delay (it) & Du & ion (H) \\
\hline RPB3 & & & & & 4.8 & 0. & & & 30. & 4.8 & & 2.5 \\
\hline & & & & & & 0. & & & 30. & 7.3 & & 4 \\
\hline RPB2 & & & & & 4.8 & 6.4 & & & 30. & 7.3 & & 0.25 \\
\hline & & & & & & 2.3. & & & 30. & 7.55 & & 4 \\
\hline RSUR2 & & & & & 5 & 5.2 & & & 10. & 12 & & 3 \\
\hline
\end{tabular}


4.4.3 Inventory Released Compared to the Shutdown Core Inventory for the Two Modified BWR Source Terms

\begin{tabular}{|c|c|c|c|c|c|c|}
\hline Isotope & Group & $\begin{array}{l}\text { Core Inventory } \\
\text { (Ci) }\end{array}$ & $\begin{array}{c}\text { MPB2 } \\
\text { (Ci) }\end{array}$ & $\begin{array}{c}\text { MPB2 } \\
\text { Fraction }\end{array}$ & $\begin{array}{l}\text { MPB3 } \\
\text { (Ci) }\end{array}$ & $\begin{array}{c}\text { MPB3 } \\
\text { Fraction }\end{array}$ \\
\hline CO-58 & 6 & $5.8 E+05$ & $1.0 E+03$ & $1.8 E-03$ & $2.0 \mathrm{E}+02$ & $3.5 E-04$ \\
\hline CO-60 & 6 & $7.0 \mathrm{E}+05$ & $1.3 E+03$ & $1.8 \mathrm{E}-03$ & $2.4 E+02$ & $3.5 E-04$ \\
\hline KR-85 & 1 & $9.5 E+05$ & $9.5 E+05$ & $1.0 \mathrm{E}+00$ & $9.5 E+05$ & $1.0 E+\infty 0$ \\
\hline KR-85M & 1 & $3.5 E+07$ & $1.1 \mathrm{E}+07$ & $3.2 \mathrm{E}-01$ & $1.5 E+07$ & $4.4 E-01$ \\
\hline KR-87 & 1 & $6.3 E+07$ & $1.1 E+06$ & $1.8 \mathrm{E}-02$ & $3.7 E+06$ & 5.9E-02 \\
\hline KR-88 & 1 & $8.5 E+07$ & $1.4 E+07$ & $1.6 \mathrm{E}-01$ & $2.3 E+07$ & $2.7 E-01$ \\
\hline RB-86 & 3 & $5.3 E+04$ & $2.3 E+03$ & 4.4E-02 & $1.3 E+03$ & $2.5 E-02$ \\
\hline SR-89 & 5 & $1.1 E+08$ & $4.3 E+06$ & 4.1E-02 & $2.2 E+06$ & 2.0E-02 \\
\hline SR-90 & 5 & $7.5 E+06$ & $3.0 \mathrm{E}+05$ & 4.1E-02 & $1.5 E+05$ & $2.0 \mathrm{E}-02$ \\
\hline SR-91 & 5 & $1.4 E+08$ & $3.2 \mathrm{E}+06$ & $2.4 E-02$ & $1.7 E+06$ & $1.2 \mathrm{E}-02$ \\
\hline SR-92 & 5 & $1.4 E+08$ & $8.5 E+05$ & $6.0 \mathrm{E}-03$ & $4.6 E+05$ & $3.2 E-03$ \\
\hline$Y-90$ & 7 & $8.0 E+06$ & $4.8 E+04$ & $6.0 \mathrm{E}-03$ & $2.3 E+04$ & $2.8 E-03$ \\
\hline Y-91 & 7 & $1.3 \mathrm{E}+08$ & $4.4 E+05$ & $3.4 E-03$ & $2.0 \mathrm{E}+05$ & $1.6 \mathrm{E}-03$ \\
\hline Y-92 & 7 & $1.4 E+08$ & $1.7 \mathrm{E}+06$ & $1.2 \mathrm{E}-02$ & $8.7 E+05$ & $6.1 E-03$ \\
\hline Y-93 & 7 & $1.6 \mathrm{E}+08$ & $3.2 E+05$ & $2.0 E-03$ & $1.5 E+05$ & $9.2 \mathrm{E}-04$ \\
\hline ZR-95 & 7 & $1.7 E+08$ & $5.6 E+05$ & $3.3 E-03$ & $2.5 E+05$ & $1.5 E-03$ \\
\hline ZR-97 & 7 & $1.7 E+08$ & $4.2 E+05$ & $2.4 E-03$ & $1.9 E+05$ & $1.1 \mathrm{E}-03$ \\
\hline NB-95 & 7 & $1.6 E+08$ & $5.3 E+05$ & $3.3 E-03$ & $2.4 \mathrm{E}+05$ & $1.5 E-03$ \\
\hline MO-99 & 6 & $1.8 \mathrm{E}+08$ & $3.1 \mathrm{E}+05$ & $1.7 E-03$ & $6.0 E+04$ & $3.3 E-04$ \\
\hline TC-99M & 6 & $1.6 E+08$ & $3.0 \mathrm{E}+05$ & $1.9 E-03$ & $5.9 E+04$ & $3.7 E-04$ \\
\hline RU-103 & 6 & $1.4 \mathrm{E}+08$ & $2.5 E+05$ & $1.8 E-03$ & $4.9 E+04$ & $3.5 E-04$ \\
\hline RU-105 & 6 & $9.3 E+07$ & $5.3 E+04$ & 5.7E-04 & $1.1 E+04$ & $1.2 \mathrm{E}-04$ \\
\hline RU-106 & 6 & $3.8 E+07$ & $6.9 E+04$ & $1.8 \mathrm{E}-03$ & $1.3 E+04$ & $3.5 E-04$ \\
\hline RH-105 & 6 & $7.0 \mathrm{E}+07$ & $1.2 \mathrm{E}+05$ & $1.7 \mathrm{E}-03$ & $2.4 \mathrm{E}+04$ & $3.4 E-04$ \\
\hline SB-127 & 4 & $8.8 E+06$ & $2.6 E+05$ & $3.0 \mathrm{E}-02$ & $1.7 E+05$ & $1.9 \mathrm{E}-02$ \\
\hline SB-129 & 4 & $3.1 E+07$ & $2.9 E+05$ & $9.4 E-03$ & $2.0 E+05$ & $6.4 E-03$ \\
\hline TE-127 & 4 & $8.6 E+06$ & $2.7 E+05$ & 3.1E-02 & $1.7 E+05$ & $2.0 \mathrm{E}-02$ \\
\hline TE-127M & 4 & $1.2 E+06$ & $3.6 E+04$ & 3.1E-02 & $2.3 E+04$ & $2.0 \mathrm{E}-02$ \\
\hline TE-129 & 4 & $2.9 E+07$ & $3.9 E+05$ & $1.4 \mathrm{E}-02$ & $2.6 E+05$ & $9.2 E-03$ \\
\hline TE-129M & 4 & $7.6 E+06$ & $2.4 E+05$ & 3.1E-02 & $1.5 \mathrm{E}+05$ & $2.0 \mathrm{E}-02$ \\
\hline
\end{tabular}


4 Results

Table 4.4.3 (Continued)

\begin{tabular}{|c|c|c|c|c|c|c|}
\hline Isotope & Group & $\begin{array}{l}\text { Core Inventory } \\
\text { (Ci) }\end{array}$ & $\begin{array}{l}\text { MPB2 } \\
\text { (Ci) }\end{array}$ & $\begin{array}{c}\text { MPB2 } \\
\text { Fraction }\end{array}$ & $\begin{array}{c}\text { MPB3 } \\
(\mathbf{C i})\end{array}$ & $\begin{array}{c}\text { MPB3 } \\
\text { Fraction }\end{array}$ \\
\hline TE-131M & 4 & $1.5 E+07$ & $3.8 \mathrm{E}+05$ & $2.6 \mathrm{E}-02$ & $2.5 \mathrm{E}+05$ & $1.7 \mathrm{E}-02$ \\
\hline TE-132 & 4 & $1.4 \mathrm{E}+08$ & $4.2 \mathrm{E}+06$ & 2.9E-02 & $2.7 E+06$ & $1.9 \mathrm{E}-02$ \\
\hline I-131 & 2 & $9.8 \mathrm{E}+07$ & $4.7 E+06$ & 4.8E-02 & $2.5 E+06$ & $2.6 \mathrm{E}-02$ \\
\hline $\mathrm{I}-132$ & 2 & $1.4 \mathrm{E}+08$ & $4.6 \mathrm{E}+06$ & $3.2 \mathrm{E}-02$ & $2.8 E+06$ & $2.0 \mathrm{E}-02$ \\
\hline I-133 & 2 & $2.1 \mathrm{E}+08$ & $7.9 E+06$ & 3.9E-02 & $4.3 E+06$ & 2.1E-02 \\
\hline I-134 & 2 & $2.3 E+08$ & $2.9 E+04$ & $1.3 \mathrm{E}-04$ & $2.5 E+04$ & 1.1E-04 \\
\hline I-135 & 2 & $1.9 \mathrm{E}+08$ & $4.4 E+06$ & 2.2E-02 & $2.4 \mathrm{E}+06$ & $1.2 \mathrm{E}-02$ \\
\hline XE-133 & 1 & $2.1 \mathrm{E}+08$ & $2.0 \mathrm{E}+08$ & $9.6 \mathrm{E}-01$ & $2.0 \mathrm{E}+08$ & $9.7 \mathrm{E}-01$ \\
\hline XE-135 & 1 & $4.9 \mathrm{E}+07$ & $3.1 E+07$ & $6.3 E-01$ & $3.4 E+07$ & $7.0 \mathrm{E}-01$ \\
\hline CS-134 & 3 & $1.6 \mathrm{E}+07$ & $7.2 E+05$ & 4.5E-02 & $4.1 E+05$ & $2.6 \mathrm{E}-02$ \\
\hline CS-136 & 3 & $4.3 E+06$ & $1.9 E+05$ & 4.4E-02 & $1.1 E+05$ & $2.5 \mathrm{E}-02$ \\
\hline CS-137 & 3 & $9.6 E+06$ & $4.3 E+05$ & 4.4E-02 & $2.5 \mathrm{E}+05$ & $2.6 \mathrm{E}-02$ \\
\hline BA-139 & 9 & $1.9 E+08$ & $1.4 \mathrm{E}+05$ & 7.2E-04 & $1.1 E+05$ & $5.7 E-04$ \\
\hline BA-140 & 9 & $1.9 \mathrm{E}+08$ & $5.7 E+06$ & 3.1E-02 & $3.8 \mathrm{E}+06$ & $2.0 \mathrm{E}-02$ \\
\hline LA-140 & 7 & $1.9 \mathrm{E}+08$ & $1.3 E+06$ & $6.6 \mathrm{E}-03$ & $7.0 \mathrm{E}+05$ & 3.7E-03 \\
\hline LA-141 & 7 & $1.8 \mathrm{E}+08$ & $1.5 E+05$ & 8.8E-04 & $7.4 \mathrm{E}+04$ & $4.2 \mathrm{E}-04$ \\
\hline LA-142 & 7 & $1.7 \mathrm{E}+08$ & $2.1 E+04$ & $1.2 \mathrm{E}-04$ & $1.1 E+04$ & $6.4 \mathrm{E}-05$ \\
\hline CE-141 & 8 & $1.7 E+08$ & $7.3 E+05$ & 4.3E-03 & $3.5 \mathrm{E}+05$ & 2.0E-03 \\
\hline CE-143 & 8 & $1.7 \mathrm{E}+08$ & $6.1 E+05$ & 3.7E-03 & $2.9 E+05$ & $1.8 \mathrm{E}-03$ \\
\hline CE-144 & 8 & $1.1 E+08$ & $4.7 E+05$ & 4.3E-03 & $2.3 E+05$ & $2.0 \mathrm{E}-03$ \\
\hline PR-143 & 7 & $1.6 \mathrm{E}+08$ & $5.4 E+05$ & 3.3E-03 & $2.5 E+05$ & $1.5 E-03$ \\
\hline ND-147 & 7 & $7.2 E+07$ & $2.3 E+05$ & $3.2 \mathrm{E}-03$ & $1.1 E+05$ & $1.5 \mathrm{E}-03$ \\
\hline NP-239 & 8 & $2.2 E+09$ & $8.5 E+06$ & $3.9 \mathrm{E}-03$ & $4.0 E+06$ & $1.9 \mathrm{E}-03$ \\
\hline PU-238 & 8 & $1.5 E+05$ & $6.5 E+02$ & 4.3E-03 & $3.1 E+02$ & $2.1 E-03$ \\
\hline PU-239 & 8 & $3.8 \mathrm{E}+04$ & $1.6 \mathrm{E}+02$ & 4.3E-03 & $7.8 \mathrm{E}+01$ & $2.1 \mathrm{E}-03$ \\
\hline PU-240 & 8 & $4.8 \mathrm{E}+04$ & $2.0 \mathrm{E}+02$ & 4.3E-03 & $9.8 \mathrm{E}+01$ & $2.0 \mathrm{E}-03$ \\
\hline PU-241 & 8 & $8.2 E+06$ & $3.5 E+04$ & 4.3E-03 & $1.7 E+04$ & 2.1E-03 \\
\hline AM-241 & 7 & $8.3 E+03$ & $2.8 \mathrm{E}+01$ & 3.3E-03 & $1.3 E+01$ & $1.5 \mathrm{E}-03$ \\
\hline CM-242 & 7 & $2.2 E+06$ & $7.3 E+03$ & 3.3E-03 & $3.3 E+03$ & $1.5 \mathrm{E}-03$ \\
\hline CM-244 & 7 & $1.2 \mathrm{E}+05$ & $3.9 E+02$ & 3.3E-03 & $1.8 \mathrm{E}+02$ & $1.5 \mathrm{E}-03$ \\
\hline
\end{tabular}


Table 4.4.4 Inventory Released Compared to the Shutdown Core Inventory for the Modified PWR Source Term

\begin{tabular}{|c|c|c|c|c|}
\hline Isotope & Group & $\begin{array}{l}\text { Core Inventory } \\
\text { (Ci) }\end{array}$ & $\begin{array}{c}\text { MSUR2 } \\
\text { (Ci) }\end{array}$ & $\begin{array}{c}\text { MSUR2 } \\
\text { Fraction }\end{array}$ \\
\hline CO-58 & 6 & $9.7 E+05$ & $9.6 \mathrm{E}+02$ & $9.9 E-04$ \\
\hline$C O-60$ & 6 & $7.4 E+05$ & $7.4 \mathrm{E}+02$ & 1.0E-03 \\
\hline KR-85 & 1 & $7.5 \mathrm{E}+05$ & $7.5 \mathrm{E}+05$ & $1.0 \mathrm{E}+00$ \\
\hline KR-85M & 1 & $3.5 E+07$ & $5.5 \mathrm{E}+06$ & $1.6 \mathrm{E}-01$ \\
\hline KR-87 & 1 & $6.4 \mathrm{E}+07$ & $9.0 \mathrm{E}+04$ & $1.4 \mathrm{E}-03$ \\
\hline KR-88 & 1 & $8.6 \mathrm{E}+07$ & $4.4 E+06$ & 5.1E-02 \\
\hline RB-86 & 3 & $5.7 \mathrm{E}+04$ & $8.4 \mathrm{E}+02$ & 1.5E-02 \\
\hline SR-89 & 5 & $1.1 \mathrm{E}+08$ & $3.2 E+05$ & 3.0E-03 \\
\hline SR-90 & 5 & $5.8 \mathrm{E}+06$ & $1.8 \mathrm{E}+04$ & 3.0E-03 \\
\hline SR-91 & 5 & $1.4 \mathrm{E}+08$ & $1.7 \mathrm{E}+05$ & $1.2 \mathrm{E}-03$ \\
\hline SR-92 & 5 & $1.4 \mathrm{E}+0$ & $2.0 \mathrm{E}+04$ & $1.4 \mathrm{E}-04$ \\
\hline$Y-90$ & 7 & $6.3 E+06$ & $4.3 E+03$ & $6.9 \mathrm{E}-04$ \\
\hline$Y-91$ & 7 & $1.3 E+08$ & $5.4 E+04$ & 4.1E-04 \\
\hline$Y-92$ & 7 & $1.5 \mathrm{E}+08$ & $7.5 \mathrm{E}+04$ & $5.2 \mathrm{E}-04$ \\
\hline$Y-93$ & 7 & $1.6 \mathrm{E}+08$ & $2.9 E+04$ & $1.8 \mathrm{E}-04$ \\
\hline ZR-95 & 7 & $1.7 \mathrm{E}+08$ & $6.6 \mathrm{E}+04$ & 4.0E-04 \\
\hline ZR-97 & 7 & $1.7 \mathrm{E}+08$ & $4.2 E+04$ & 2.4E-04 \\
\hline NB-95 & 7 & $1.6 \mathrm{E}+08$ & $6.3 E+04$ & $4.0 \mathrm{E}-04$ \\
\hline MO-99 & 6 & $1.8 \mathrm{E}+08$ & $1.6 E+05$ & 8.8E-04 \\
\hline TC-99M & 6 & $1.6 E+08$ & $1.7 E+05$ & $1.1 \mathrm{E}-03$ \\
\hline RU-103 & 6 & $1.4 E+08$ & $1.4 \mathrm{E}+05$ & 9.9E-04 \\
\hline RU-105 & 6 & $8.9 E+07$ & $1.4 \mathrm{E}+04$ & $1.5 \mathrm{E}-04$ \\
\hline RU-106 & 6 & $3.1 E+07$ & $3.1 E+04$ & $1.0 \mathrm{E}-03$ \\
\hline RH-105 & 6 & $6.2 \mathrm{E}+07$ & $5.7 E+04$ & 9.2E-0.4 \\
\hline SB-127 & 4 & $8.4 E+06$ & $3.5 E+05$ & 4.1E-02 \\
\hline SB-129 & 4 & $3.0 \mathrm{E}+07$ & $2.0 \mathrm{E}+05$ & $6.6 \mathrm{E}-03$ \\
\hline TE-127 & 4 & $8.1 E+06$ & $3.6 \mathrm{E}+05$ & 4.5E-02 \\
\hline TE-127M & 4 & $1.1 E+06$ & $4.8 \mathrm{E}+04$ & $4.5 \mathrm{E}-02$ \\
\hline TE-129 & 4 & $2.8 \mathrm{E}+07$ & $2.7 \mathrm{E}+05$ & $9.6 \mathrm{E}-03$ \\
\hline
\end{tabular}


Table 4.4.4 (Continued)

\begin{tabular}{|c|c|c|c|c|}
\hline Isotope & Group & $\begin{array}{l}\text { Core Inventory } \\
\text { (Ci) }\end{array}$ & $\begin{array}{c}\text { MSUR2 } \\
\text { (Ci) }\end{array}$ & $\begin{array}{l}\text { MSUR2 } \\
\text { Fraction }\end{array}$ \\
\hline TE-129M & 4 & $7.4 \mathrm{E}+06$ & $3.3 E+05$ & 4.4E-02 \\
\hline TE-131M & 4 & $1.4 \mathrm{E}+07$ & $4.8 \mathrm{E}+05$ & 3.4E-02 \\
\hline TE-132 & 4 & $1.4 \mathrm{E}+08$ & $5.7 \mathrm{E}+06$ & 4.0E-02 \\
\hline I-131 & 2 & $9.7 E+07$ & $2.8 E+06$ & 2.9E-02 \\
\hline I-132 & 2 & $1.4 E+08$ & $5.8 \mathrm{E}+06$ & 4.1E-02 \\
\hline I-133 & 2 & $2.0 \mathrm{E}+08$ & $4.1 \mathrm{E}+06$ & $2.0 \mathrm{E}-02$ \\
\hline I-134 & 2 & $2.2 \mathrm{E}+08$ & $5.1 \mathrm{E}+02$ & 2.3E-06 \\
\hline I-135 & 2 & $1.9 E+08$ & $1.6 E+06$ & 8.5E-03 \\
\hline$X E-133$ & 1 & $2.0 E+08$ & $1.9 \mathrm{E}+08$ & $9.4 \mathrm{E}-01$ \\
\hline XE-135 & 1 & $3.8 \mathrm{E}+07$ & $1.7 \mathrm{E}+07$ & $4.5 \mathrm{E}-01$ \\
\hline CS-134 & 3 & $1.3 \mathrm{E}+07$ & $2.0 \mathrm{E}+05$ & $1.5 \mathrm{E}-02$ \\
\hline CS-136 & 3 & $4.0 \mathrm{E}+06$ & $5.8 \mathrm{E}+04$ & $1.5 \mathrm{E}-02$ \\
\hline CS-137 & 3 & $7.3 E+06$ & $1.1 \mathrm{E}+05$ & $1.5 \mathrm{E}-02$ \\
\hline BA-139 & 9 & $1.9 E+08$ & $1.4 E+03$ & $7.4 \mathrm{E}-06$ \\
\hline BA-140 & 9 & $1.9 E+08$ & $5.5 E+05$ & $2.9 \mathrm{E}-03$ \\
\hline LA-140 & 7 & $1.9 E+08$ & $1.7 \mathrm{E}+05$ & 8.7E-04 \\
\hline LA-141 & 7 & $1.8 \mathrm{E}+08$ & $8.5 E+03$ & 4.8E-05 \\
\hline LA-142 & 7 & $1.7 E+08$ & $3.6 \mathrm{E}+02$ & 2.1E-06 \\
\hline CE-141 & 8 & 1.7E+08 & $6.8 \mathrm{E}+04$ & 4.0E-04 \\
\hline CE-143 & 8 & $1.7 E+08$ & $5.1 E+04$ & 3.1E-04 \\
\hline CE-144 & 8 & $1.0 \mathrm{E}+08$ & $4.1 E+04$ & 4.0E-04 \\
\hline PR-143 & 7 & $1.6 \mathrm{E}+08$ & $6.5 \mathrm{E}+04$ & $4.0 \mathrm{E}-04$ \\
\hline ND-147 & 7 & $7.3 E+07$ & $2.8 \mathrm{E}+04$ & $3.9 \mathrm{E}-04$ \\
\hline NP-239 & 8 & $1.9 E+09$ & $6.7 E+05$ & 3.5E-04 \\
\hline PU-238 & 8 & $1.1 \mathrm{E}+05$ & $4.4 \mathrm{E}+01$ & 4.0E-04 \\
\hline PU-239 & 8 & $2.5 E+04$ & $1.0 \mathrm{E}+01$ & $4.0 \mathrm{E}-04$ \\
\hline PU-240 & 8 & $3.1 E+04$ & $1.3 E+01$ & $4.0 \mathrm{E}-04$ \\
\hline PU-241 & 8 & $5.3 E+06$ & $2.1 E+03$ & 4.0E-04 \\
\hline AM-241 & 7 & $3.5 \mathrm{E}+03$ & $1.4 \mathrm{E}+\infty 0$ & $4.0 \mathrm{E}-04$ \\
\hline CM-242 & 7 & $1.3 E+06$ & $5.3 \mathrm{E}+02$ & $4.0 \mathrm{E}-04$ \\
\hline CM-244 & 7 & $7.8 \mathrm{E}+04$ & $3.1 \mathrm{E}+01$ & $4.0 \mathrm{E}-04$ \\
\hline
\end{tabular}


Table 4.4.5 Inventory Released Compared to the Shutdown Core Inventory for the Two Modified BWR Source Terms, Listed by Isotope Grouping

\begin{tabular}{|c|c|c|c|c|c||}
\hline Group & $\begin{array}{c}\text { Core Inventory } \\
(\mathrm{Ci})\end{array}$ & $\begin{array}{c}\text { MPB2 } \\
(\mathrm{Ci})\end{array}$ & $\begin{array}{c}\text { MPB2 } \\
\text { Fraction }\end{array}$ & $\begin{array}{c}\text { MPB3 } \\
(\mathrm{Ci})\end{array}$ & $\begin{array}{c}\text { MPB3 } \\
\text { Fraction }\end{array}$ \\
\hline 1 & $4.4 \mathrm{E}+08$ & $2.6 \mathrm{E}+08$ & $5.8 \mathrm{E}-01$ & $2.8 \mathrm{E}+08$ & $6.3 \mathrm{E}-01$ \\
\hline 2 & $8.7 \mathrm{E}+08$ & $2.2 \mathrm{E}+07$ & $2.5 \mathrm{E}-02$ & $1.2 \mathrm{E}+07$ & $1.4 \mathrm{E}-02$ \\
\hline 3 & $3.0 \mathrm{E}+07$ & $1.3 \mathrm{E}+06$ & $4.4 \mathrm{E}-02$ & $7.7 \mathrm{E}+05$ & $2.6 \mathrm{E}-02$ \\
\hline 4 & $2.4 \mathrm{E}+08$ & $6.0 \mathrm{E}+06$ & $2.5 \mathrm{E}-02$ & $3.9 \mathrm{E}+06$ & $1.6 \mathrm{E}-02$ \\
\hline 5 & $3.9 \mathrm{E}+08$ & $8.7 \mathrm{E}+06$ & $2.2 \mathrm{E}-02$ & $4.4 \mathrm{E}+06$ & $1.1 \mathrm{E}-02$ \\
\hline 6 & $6.9 \mathrm{E}+08$ & $1.1 \mathrm{E}+06$ & $1.6 \mathrm{E}-03$ & $2.2 \mathrm{E}+05$ & $3.2 \mathrm{E}-04$ \\
\hline 7 & $1.7 \mathrm{E}+09$ & $6.2 \mathrm{E}+06$ & $3.6 \mathrm{E}-03$ & $3.1 \mathrm{E}+06$ & $1.8 \mathrm{E}-03$ \\
\hline 8 & $2.6 \mathrm{E}+09$ & $1.0 \mathrm{E}+07$ & $3.9 \mathrm{E}-03$ & $4.9 \mathrm{E}+06$ & $1.9 \mathrm{E}-03$ \\
\hline 9 & $3.8 \mathrm{E}+08$ & $5.9 \mathrm{E}+06$ & $1.6 \mathrm{E}-02$ & $3.9 \mathrm{E}+06$ & $1.0 \mathrm{E}-02$ \\
\hline
\end{tabular}


Table 4.4.6 Inventory Released Compared to the Shutdown Core Inventory for the Modified PWR Source Term, Listed by Isotope Grouping

\begin{tabular}{|c|c|c|c||}
\hline Group & $\begin{array}{c}\text { Core Inventory } \\
(\mathrm{Ci})\end{array}$ & $\begin{array}{c}\mathrm{MPB} 2 \\
(\mathrm{Ci})\end{array}$ & $\begin{array}{c}\text { MPB2 } \\
\text { Fraction }\end{array}$ \\
\hline 1 & $4.3 \mathrm{E}+08$ & $2.2 \mathrm{E}+08$ & $5.1 \mathrm{E}-01$ \\
\hline 2 & $8.6 \mathrm{E}+08$ & $1.4 \mathrm{E}+07$ & $1.7 \mathrm{E}-02$ \\
\hline 3 & $2.4 \mathrm{E}+07$ & $3.6 \mathrm{E}+05$ & $1.5 \mathrm{E}-02$ \\
\hline 4 & $2.4 \mathrm{E}+08$ & $7.7 \mathrm{E}+06$ & $3.3 \mathrm{E}-02$ \\
\hline 5 & $4.0 \mathrm{E}+08$ & $5.3 \mathrm{E}+05$ & $1.3 \mathrm{E}-03$ \\
\hline 6 & $6.6 \mathrm{E}+08$ & $5.7 \mathrm{E}+05$ & $8.6 \mathrm{E}-04$ \\
\hline 7 & $1.7 \mathrm{E}+09$ & $6.0 \mathrm{E}+05$ & $3.5 \mathrm{E}-04$ \\
\hline 8 & $2.4 \mathrm{E}+09$ & $8.4 \mathrm{E}+05$ & $3.5 \mathrm{E}-04$ \\
\hline 9 & $3.8 \mathrm{E}+08$ & $5.5 \mathrm{E}+05$ & $1.5 \mathrm{E}-03$ \\
\hline
\end{tabular}


Table 4.4.7 The Mean Number of Individuals Exceeding Dose Thresholds to the Three Organs Used in the Early Fatality Health Effects Model

\begin{tabular}{|l|l|}
\hline Source Term: MPB2 & Mean Number Exceeding Dose Threshold \\
\hline Red Marrow > 150 Rem & $0.95 * 12.8+0.05 * 39.8=14.2$ \\
\hline Lungs > 500 Rem & $0.95 * 4.75+0.05 * 16.3=5.32$ \\
\hline Lower Large Intestine > 800 Rem & $0.95 * 0.478+0.05 * 2.24=0.566$ \\
\hline Source Term: MPB3 & $0.95 * 10.5+0.05 * 25.1=11.3$ \\
\hline Red Marrow > 150 Rem & $0.95 * 3.49+0.05 * 8.82=3.76$ \\
\hline Lungs > 500 Rem & $0.95 * 0.439+0.05 * 2.01=0.518$ \\
\hline Lower Large Intestine > 800 Rem & $0.95 * 7.04+0.05 * 59.8=9.68$ \\
\hline Source Term: MSUR2 & $0.95 * 1.93+0.05 * 13.8=2.53$ \\
\hline Red Marrow > 150 Rem & $0.95 * 0.174+0.05 * 2.37=0.284$ \\
\hline Lungs > 500 Rem & Lower Large Intestine > 800 Rem \\
\hline
\end{tabular}




\subsection{Equivalent Curies}

The final set of calculations reported bere was to determine the amount, in curies, released of a particular isotope. lodine-131 the c would have the potential to cause one early fatality in the mean for various sets of assumptions regarding population, meteorology, exclusion boundary, power level, emergency response, time of release, warning time, etc. Some of the cases overlap those analyzed in sections 4.1, 4.2, 4.3 and 4.4. The objective is to establish the equivalence, under the same set of assumptions, between the amount of 1-131 released and the amounts of all of the nine radionuclide groups released required to result in one mean early fatality as estimated by MACCS.

The specific assumptions for the two hypothetical base cases which were developed are shown in Table 4.5.1. Case 1 assumed a time of release two hours after accident recognition, a release duration of one hour, and a delay time of two bours for the emergency response. In Case 2, the corresponding times were assumed to be four, two, and four hours, respectively. Both cases assumed a population density of 1000 people per sq. mile, exclusion boundary distance of 0.4 mile, 80 th percentile weather, reactor power level of $3800 \mathrm{MWth}$, and an emergency response consisting of 100 percent of the population within 10 miles sheltering for 12 hours followed by an instant relocation.

The following sets of calculations were carried out:

(1) The first set of calculations based on Case 1 and Case 2 considered only I-131 releases; the inventory of all the other isotopes was set equal to zero. The results for the number of MACCS estimated early fatalities (mean and 95th percentile) within one mile of the exclusion area boundary are shown in Tables 4.5.2 and 4.5.3 for Cases 1 and 2, respectively as a function of the I-131 inventory released. The mean values of the early fatalities for both cases are also depicted graphically in Figure 4.5.1. For these calculations, the power level (or equivalently the inventory of I-131), was scaled from 0.2 to 1.0 times the $3800 \mathrm{MWth}$ level. As Table 4.5.2 shows, the estimated number of mean early fatalities varies from 0.03 to 12.3 over this range of power levels for Case 1. The I-131 inventory released needed to have the potential to lead to one mean early fatality corresponds to between 40 and 50 percent of the basic $3800 \mathrm{MWth}$ reactor inventory of I-131 which is equivalent to about 4 to 5 percent of the total iodine inventory at shutdown. For Case 2, as shown in Table 4.5.3, the corresponding fraction of the I-131 inventory release that potentially leads to one mean early fatality lies between 50 and 60 percent of the I-131 inventory of a 3800 MWth reactor.

(2) Tables 4.5.4 and 4.5.5 are based on I-131 release fractions which have the potential to lead to one early fatality within one mile from the exclusion area boundary in Cases $1(0.428)$ and $2(0.536)$, respectively. For these releases, the number of people exceeding the threshold dose for early fatality to each of the critical organs (in the MACCS early health model) is shown.

(3) The next set of calculations, displayed in Tables 4.5.6, 4.5.7, and Figure 4.5.2, were designed to evaluate the site boundary dose as a function of the I-131 released inventory for both the cases, 1 and 2 . Assuming one person located at the site boundary in each of the 16 angular sectors, the population weighted risk of early fatality was calculated (this is the number of early fatalities divided by the total population "at risk" which is equal to 16 in this particular case). For almost all of the releases, the dose at the boundary is much higher than the LD50' dose as shown in Figure 4.5.2. However, for most weather conditions, the plume at the site

'LD50 is defined as the dose to a given organ that will result in a $50 \%$ probability of causing death from the exposure. This value is 350 rem to the Red Marrow, 1,000 rems to the lungs and 1,500 rems to the lower large intestine. 
boundary spreads over only a fraction of a sector, and since there is only one person in each sector, the code calculates a fractional fatality in most casos. (If one early fatality occurred in each case, the population weighted risk would be identical to $1 / 16$ or 0.0625 ). That is why Tables 4.5.6 and 4.5.7 and Figure 4.5.3 show mean values of the population weighted risk below 0.06 in all cases. ${ }^{2}$ A comparison can be made between the large release frequency of $10^{-6}$ per year and the early fatality QHO. For a population weighted risk of $0.06(1 / 16)$, the frequency implied by the QHO is: $5 \times 10^{-7} / 0.06$ per year, or, approximately, $10^{-5}$ per year. The large release guideline is thus approximately one order of magnitude more conservative than the QHO.

(4) In the next set of calculations, the population density was reduced from 1000 to 10 people/sq. mile; the other inputs were the same. The results are shown in Tables 4.5.8, 4.5.9 and Figure 4.5.4 for both cases, 1 and 2. The estimated number of early fatalities scale roughly by the change in population density, i.e., reduced by about two orders of magnitude.

(5) For the final set of calculations carried out for Cases 1 and 2, the release fractions of iodine, cesium, and tellurium for a PWR inventory were adjusted to result in the potential to lead to one mean early fatality out to one mile from the exclusion boundary with the release time, etc., as assumed above. The resulting source terms are labelled as MPWR1 and MPWR2 and shown in Table 4.5.10. In addition, another source term MPWR3 was created, by allowing a hold up in containment for 12 hours before release, assuming a release duration of 12 hours and then adjusting the release fractions to obtain the potential to lead to one mean early fatality. The population exceeding the threshold dose to the critical organs are shown in Table 4.5.11 for the MPWR1, MPWR2 and MPWR3 source terms. For MPWR1 and MPWR2, the power levels (and corresponding inventories) were also reduced to $1800 \mathrm{MWth}$ and the estimated number of early fatalities were recalculated. The number of estimatod mean early fatalities was calculated to be 0.020 and 0.022 . This calculation indicates the threshold nature of the early bealth model used to estimate early fatalities.

Doses to the critical organs and by pathway are shown in the tables in Appendix B. Table B.5.1 shows the dose to the three critical organs by pathway for the I-131 releases with the Case 1 and 2 timing characteristics. Table B.5.2 displays the dose to the three critical organs by pathway for the MPWR1, MPWR2, and MPWR3 source terms shown in Table 4.5.10. The information in Tables B.5.1 and B.5.2 has been condensed into Tables B.5.3 and B.5.4 to show the relative fractions of dose by pathway to the critical organs.

For all of these source terms the red marrow dose is the dominant contributor as is emphasized by the tables showing the population exceeding the threshold doses for the early health effects model. It is interesting to note that for the I-131 release alone, the groundshine pathway is the major contributor to the red marrow dose. With the full inventory, the cloudshine is the largest contributor to the red marrow dose for the MPWRI source term, groundshine is the major contributor for the MPWR3 source term, and groundshine and cloudshine are evenly split for the MPWR2 source term.

\footnotetext{
2Because most weather conditions will not cause much lateral spread of the plume, there is a portion of the population within a sector that is not directly under the centerline portion of the plume. Therefore in order for there to be a population weighted fatality of $1 / 16$ an extremely large amount of $1-131$ must be released. Only at the 95th percentile, where extreme weather leads to more lateral dispersion of the plume, does the population weighted risk exceed $1 / 16$ by a small amount, i.e., there is a small probability of a potential to lead to an early fatality in an adjacent sector.
} 


\section{Results}

Tables 4.5.12 and 4.5.13 display the inventories assumed to be released to the environment for the MPWR1, MPWR2, and MPWR3 source terms. Table 4.5.12 shows the inventory of each radioisotope release and Table 4.5.13 displays the inventory released grouped by the standard isotope grouping. 
4 Results

Figure 4.5-1 Estimated Number of Early Fatalities Between 0.4-1.4 Miles (1000 People/Sq. Mi.) as a Function of I-131 Release

(82 ft. ht., 0 MW Energy, 80th Percentile Weather)

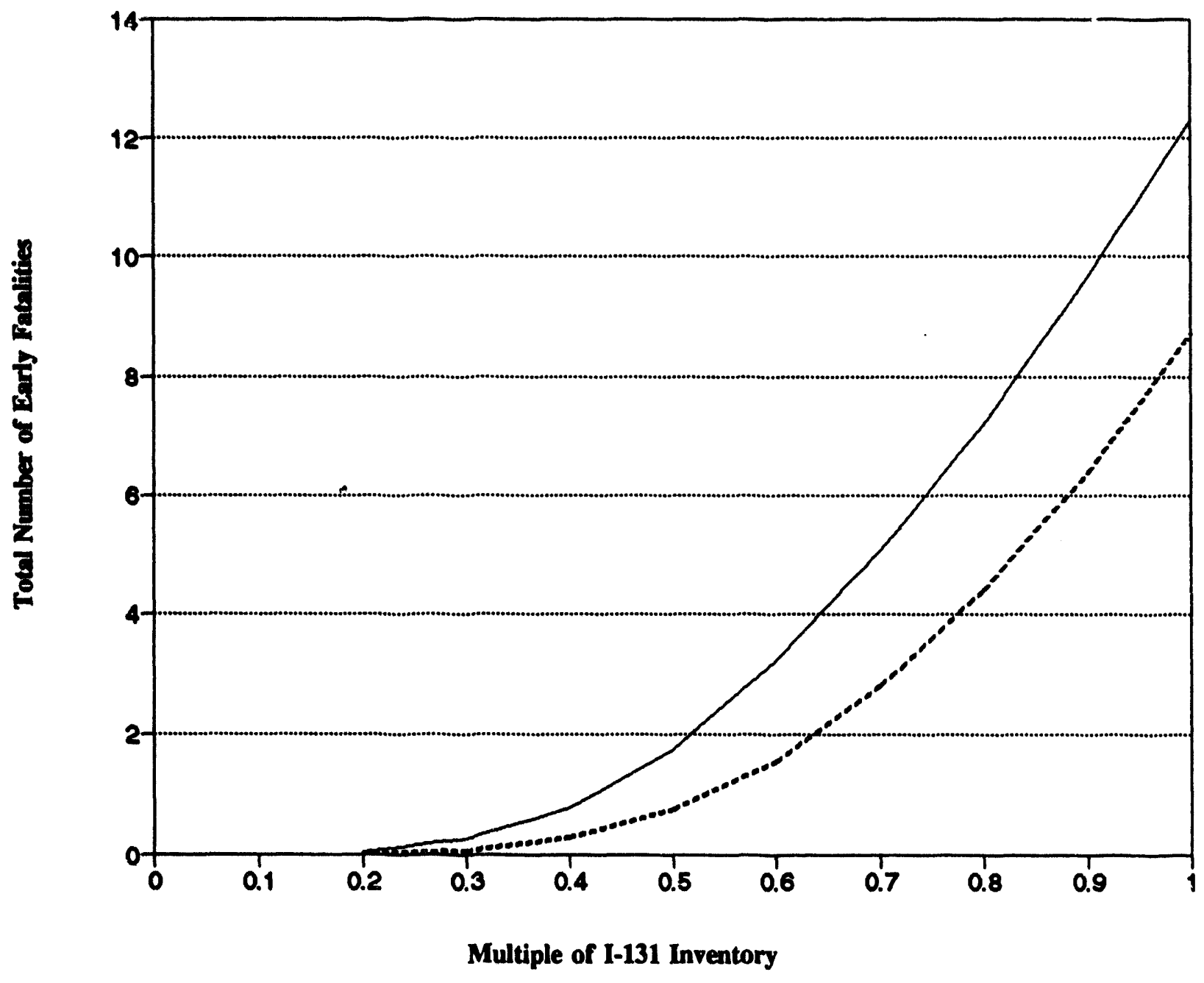

Case 1: 2 hour delay, 1 hour release duration Case 2: 4 bour delay, 2 hour release duration 
Figure 4.5-2 Whole Body Dose at Site Boundary as a Function of I-131 Release (82 ft. ht., 0 MW Energy, 80th Percentile Weather)

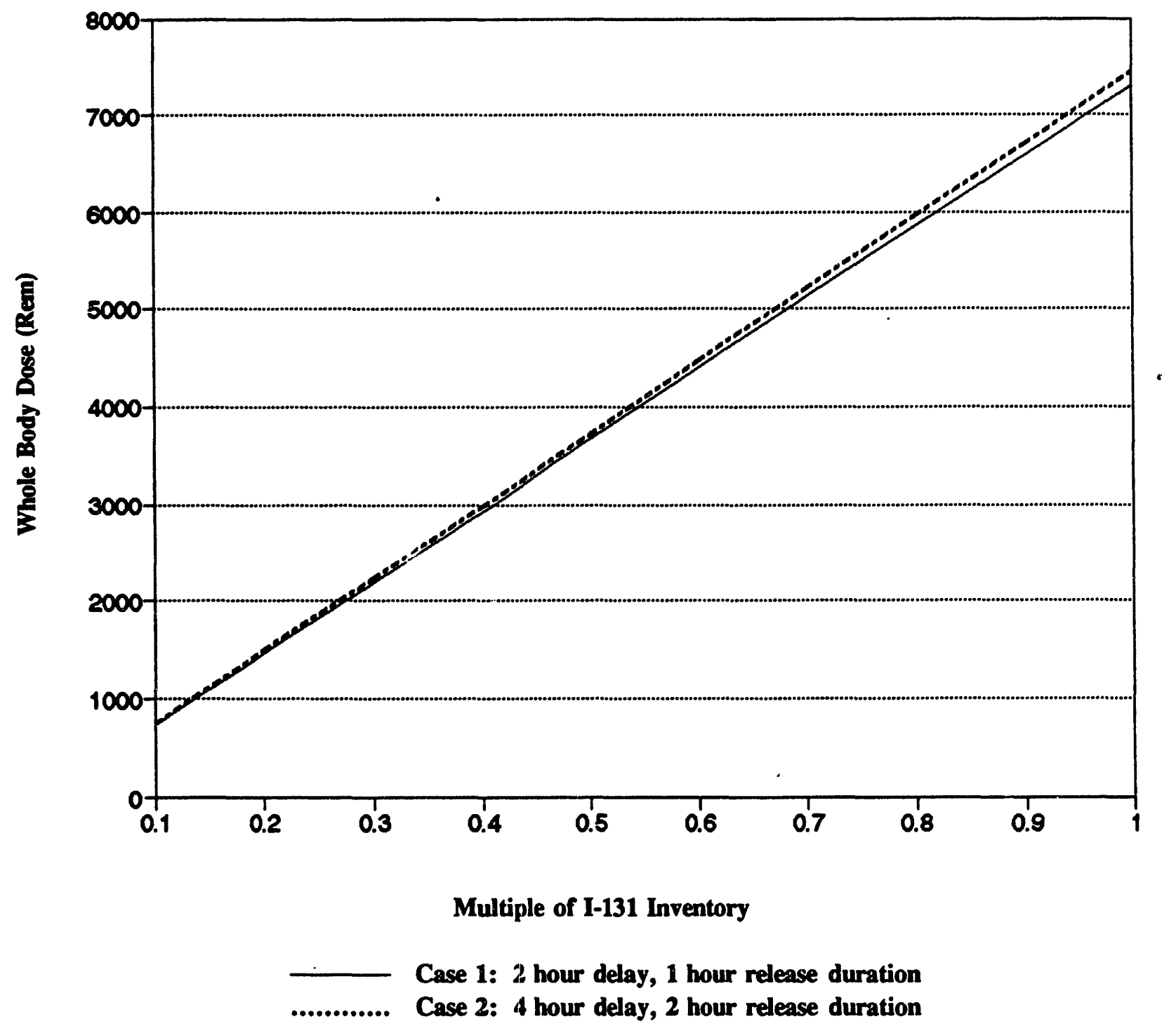


Figure 4.5-3 Population Weighted Risk for Early Fatalities at Site Boundary as a Function of I-131 Release

(82 ft. ht., 0 MW Energy, 80th Percentile Weather)

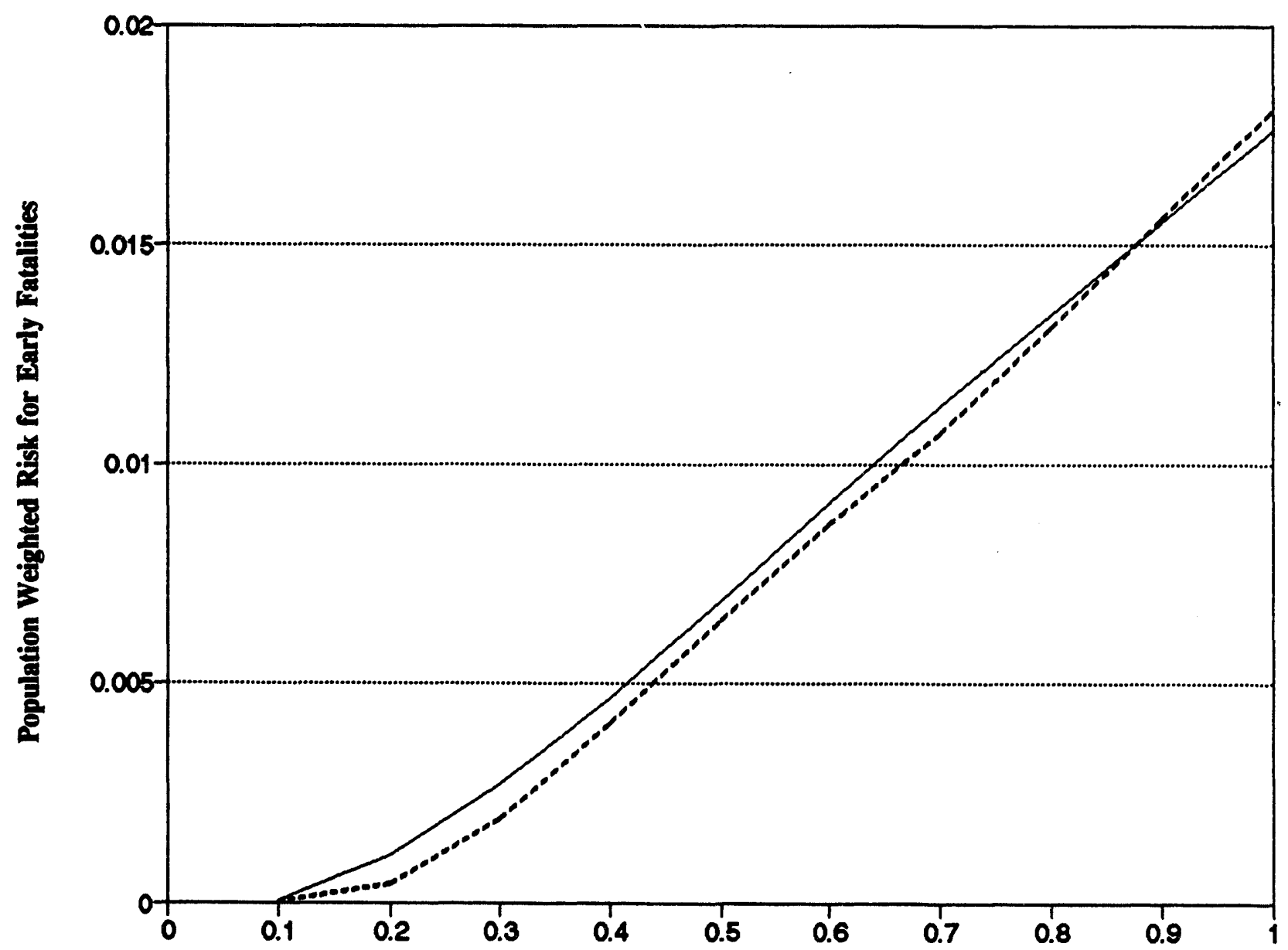

Multiple of I-131 Inventory

Case 1: 2 hour delay, 1 hour release duration

Case 2: 4 hour delay, 2 hour release duration 
Figure 4.5-4 Estimated Number of Early Fatalities Between 0.4-1.4 Miles (1000 People/Sq. Mi.) as a Function of I-131 Release

(82 ft. ht., 0 MW Energy, 80th Percentile Weather)

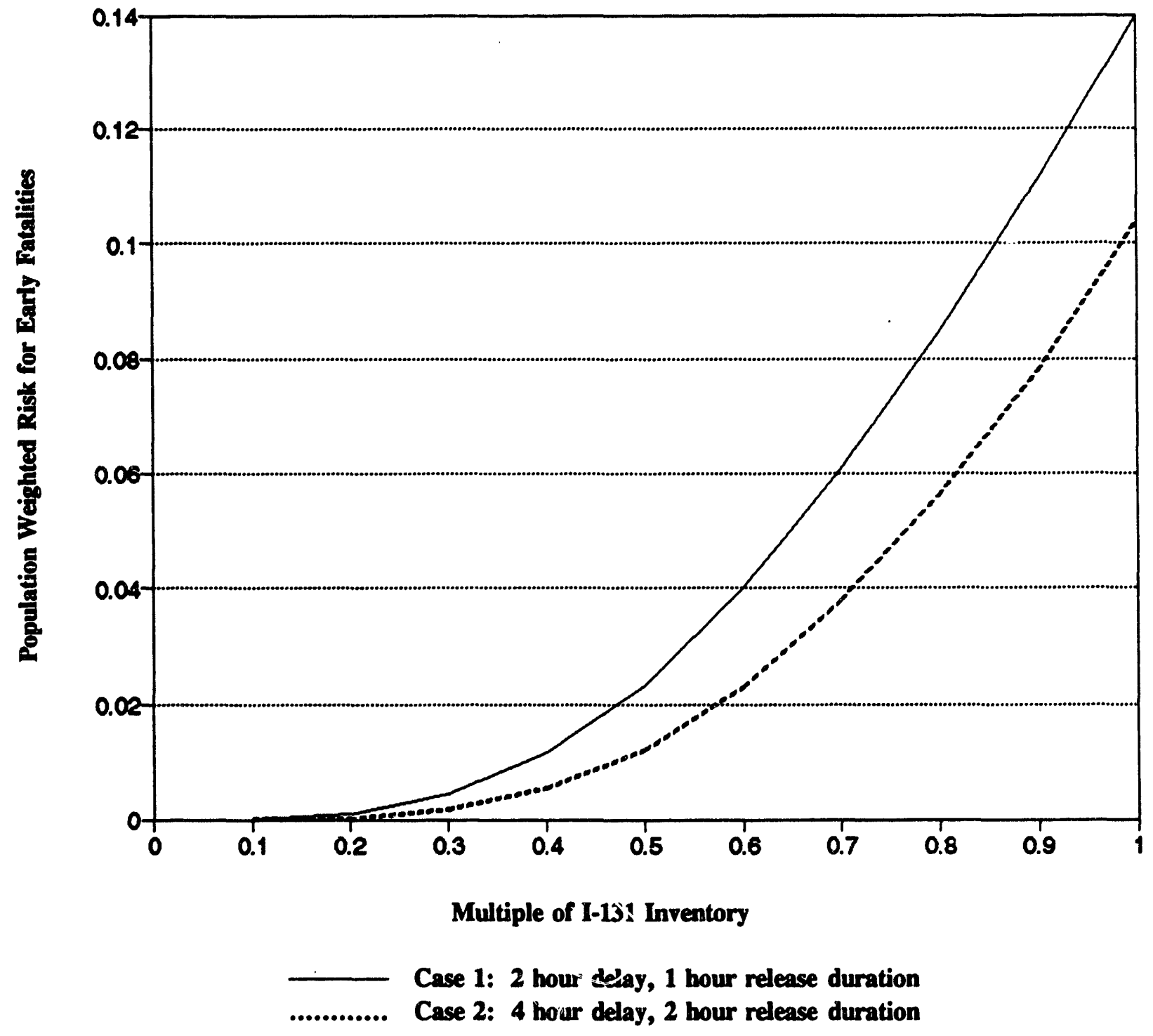


Table 4.5.1 Basic Input Assumptions for the Equivalent Curies Determination

Elevation: $82 \mathrm{Ft}$.

Energy: 0 MW

Emergency Response: $100 \%$ Sheltering for 12 Hours Followed by Relocation

Reactor Power Level: 3800 MW - I-131 Only

Population Density: Uniform 1000 People/Sq. Mi.

\begin{tabular}{||l|c|c|c|}
\hline & Time of Release & Duration & Alarm Time \\
\hline Case 1 & $2 \mathrm{Hr}$ & $1 \mathrm{Hr}$ & $2 \mathrm{Hr}$ \\
\hline Case 2 & $4 \mathrm{Hr}$ & $2 \mathrm{Hr}$ & $4 \mathrm{Hr}$ \\
\hline
\end{tabular}


Table 4.5.2 Mean Early Fatalities for I-131 Release: Case 1

Inventory: 3800 MWth PWR

$$
\text { I-131 Only }
$$

Release Height: 82 Ft.

Energy: 0 MW

Weather: 80th Percentile

Population: Uniform 1000 People/Sq. Mi.

Calculation: Early Fatalities

\begin{tabular}{|c|c|c|c|c|}
\hline & \multicolumn{2}{|c|}{ Time of Release } & Duration & Alarm Time \\
\hline Case 1 & \multicolumn{2}{|c|}{$2 \mathrm{Hrs}$} & $1 \mathrm{Hr}$ & $2 \mathrm{Hrs}$ \\
\hline \multirow{2}{*}{\multicolumn{2}{|c|}{$\begin{array}{l}\text { I-131 Release } \\
\text { Fraction }\end{array}$}} & \multicolumn{3}{|c|}{$\begin{array}{c}\text { Early Fatalities } \\
(0.4-1.4 \mathrm{Mi})\end{array}$} \\
\hline & & & ean & 95th \\
\hline \multicolumn{2}{|c|}{0.2} & & $5 E-02$ & $9.49 \mathrm{E}-02$ \\
\hline \multicolumn{2}{|c|}{0.3} & & $1 E-01$ & $1.68 \mathrm{E}+00$ \\
\hline \multicolumn{2}{|c|}{0.4} & & $4 E-01$ & $4.46 E+00$ \\
\hline \multicolumn{2}{|c|}{0.5} & & $E+\infty$ & $1.59 \mathrm{E}+01$ \\
\hline \multicolumn{2}{|c|}{0.6} & & $E+\infty$ & $1.72 \mathrm{E}+01$ \\
\hline \multicolumn{2}{|c|}{0.7} & & $E+\infty$ & $4.40 E+01$ \\
\hline \multicolumn{2}{|c|}{0.8} & & $E+\infty$ & $4.45 \mathrm{E}+01$ \\
\hline \multicolumn{2}{|c|}{0.9} & & $E+\infty$ & $6.47 E+01$ \\
\hline \multicolumn{2}{|c|}{1.0} & & $E+01$ & $9.14 E+01$ \\
\hline
\end{tabular}


Table 4.5.3 Mean Early Fatalities for I-131 Release: Case 2 Inventory: 3800 MWth PWR

$$
\text { I-131 Only }
$$

Release Height: 82 Ft.

Energy: 0 MW

Weather: 80th Percentile

Population: Uniform 1000 People/Sq. Mi.

Calculation: Early Fatalities

\begin{tabular}{|c|c|c|c|}
\hline & Time of Release & Duration & Alarm Time \\
\hline Case 2 & $4 \mathrm{Hrs}$ & $2 \mathrm{Hr}$ & $4 \mathrm{Hrs}$ \\
\hline \multirow{2}{*}{\multicolumn{2}{|c|}{$\begin{array}{l}\text { I-131 Release } \\
\text { Fraction }\end{array}$}} & \multicolumn{2}{|c|}{$\begin{array}{c}\text { Early Fatalities } \\
(0.4-1.4 \mathrm{Mi})\end{array}$} \\
\hline & & Mean & 95th \\
\hline \multicolumn{2}{|c|}{0.2} & $26 \mathrm{E}-03$ & $2.22 \mathrm{E}-02$ \\
\hline \multicolumn{2}{|c|}{0.3} & $.01 E-02$ & $2.36 \mathrm{E}-01$ \\
\hline \multicolumn{2}{|c|}{0.4} & $.97 E-01$ & $1.32 \mathrm{E}+00^{\circ}$ \\
\hline \multicolumn{2}{|c|}{0.5} & $.53 E-01$ & $3.70 \mathrm{E}+00$ \\
\hline \multicolumn{2}{|c|}{0.6} & $57 \mathrm{E}+00$ & $8.11 E+00$ \\
\hline \multicolumn{2}{|c|}{0.7} & $81 E+\infty 0$ & $1.32 \mathrm{E}+01$ \\
\hline \multicolumn{2}{|c|}{0.8} & $41 E+\infty$ & $2.24 \mathrm{E}+01$ \\
\hline \multicolumn{2}{|c|}{0.9} & $37 E+\infty$ & $3.45 \mathrm{E}+01$ \\
\hline \multicolumn{2}{|c|}{1.0} & $73 E+\infty 0$ & $5.33 E+01$ \\
\hline
\end{tabular}


Table 4.5.4 Estimated Population Exceeding Threshold Dose for I-131 Release: Case 1

Source Term: Case 1

Multiple of 3800 Mwth I-131 Inventory: 0.428

Released Inventory of All Plumes: I-131 4.1E $+7 \mathrm{Ci}$

Mean Early Fatalities (0.4-1.4 Mi): 1.00

\begin{tabular}{||l|c|c||}
\hline \multicolumn{1}{|c|}{ Organ } & $\begin{array}{c}\text { Early Acute } \\
\text { Threshold Dose }\end{array}$ & $\begin{array}{c}\text { Population Exceeding } \\
\text { Threshold }\end{array}$ \\
\hline Red Marrow & 150 Rem & 11 \\
\hline Lungs & 500 Rem & 3.62 \\
\hline Lower Large Intestine & 800 Rem & 0 \\
\hline
\end{tabular}

Table 4.5.5 Estimated Population Exceeding Threshold Dose for I-131 Release: Case 2

Source Term: Case 2

Multiple of 3800 MWth I-131 Inventory: 0.536

Released Inventory of All Plumes: I-131 $5.1 E+07 \mathrm{Ci}$

Mean Early Fatalities (0.4-1.4 Mi): 1.02

\begin{tabular}{||l|c|c||}
\hline \multicolumn{1}{|c|}{ Organ } & $\begin{array}{c}\text { Early Acute } \\
\text { Threshold Dose }\end{array}$ & $\begin{array}{c}\text { Population Exceeding } \\
\text { Threshold }\end{array}$ \\
\hline Red Marrow & 150 Rem & 16.1 \\
\hline Lungs & 500 Rem & 4.24 \\
\hline Lower Large Intestine & 800 Rem & $2.94 E-04$ \\
\hline
\end{tabular}


Table 4.5.6 Population Weighted Risk at Site Boundary for I-131 Release: Case 1

Inventory: 3800 MWth PWR

I-131 Only

Release Height: 82 Ft.

Energy: 0 MW

Weather: 80th Percentile

Population: 1 Person Per Sector Between 0.39 and 0.4 Mi.

Calculation: Population Weighted Risk for Early Fatalities

\begin{tabular}{|c|c|c|c|}
\hline & Time of Release & Duration & Alarm Time \\
\hline Case 1 & $2 \mathrm{Hrs}$ & $1 \mathrm{Hr}$ & $2 \mathrm{Hrs}$ \\
\hline \multirow{2}{*}{\multicolumn{2}{|c|}{$\begin{array}{l}\text { I-131 Release } \\
\text { Fraction }\end{array}$}} & \multicolumn{2}{|c|}{$\begin{array}{c}\text { Early Fatalities } \\
(0.4-1.4 \mathrm{Mi})\end{array}$} \\
\hline & & Mean & 95th \\
\hline \multicolumn{2}{|c|}{0.1} & $5.53 \mathrm{E}-05$ & $1.70 \mathrm{E}-04$ \\
\hline \multicolumn{2}{|c|}{0.2} & $1.07 \mathrm{E}-03$ & 6.38E-03 \\
\hline \multicolumn{2}{|c|}{0.3} & $2.74 E-03$ & $1.90 \mathrm{E}-02$ \\
\hline \multicolumn{2}{|c|}{0.4} & $4.68 E-03$ & $2.25 \mathrm{E}-02$ \\
\hline \multicolumn{2}{|c|}{0.5} & $6.85 E-03$ & $3.03 \mathrm{E}-02$ \\
\hline \multicolumn{2}{|c|}{0.6} & $9.09 E-03$ & 3.16E-02 \\
\hline \multicolumn{2}{|c|}{0.7} & $1.13 \mathrm{E}-02$ & $3.42 \mathrm{E}-02$ \\
\hline \multicolumn{2}{|c|}{0.8} & 1.34E-02 & $3.60 \mathrm{E}-02$ \\
\hline \multicolumn{2}{|c|}{0.9} & $1.55 \mathrm{E}-02$ & $3.73 E-02$ \\
\hline \multicolumn{2}{|c|}{1.0} & $1.76 \mathrm{E}-02$ & $4.28 E-02$ \\
\hline
\end{tabular}


Table 4.5.7 Population Weighted Risk at Site Boundary for I-131 Release: Case 2

Inventory: 3800 MWth PWR

$$
\text { I-131 Only }
$$

Release Height: $82 \mathrm{Ft}$.

Energy: 0 MW

Weather: 80th Percentile

Population: 1 Person Per Sector Between 0.39 and 0.4 Mi.

Calculation: Population Weighted Risk for Early Fatalities

\begin{tabular}{|c|c|c|c|}
\hline & Time of Release & Duration & Alarm Time \\
\hline Case 2 & $4 \mathrm{Hrs}$ & $2 \mathrm{Hrs}$ & $3 \mathrm{Hrs}$ \\
\hline \multirow{2}{*}{\multicolumn{2}{|c|}{$\begin{array}{l}\text { I-131 Release } \\
\text { Fraction }\end{array}$}} & \multicolumn{2}{|c|}{$\begin{array}{c}\text { Early Fatalities } \\
(0.4-1.4 \text { Mi) }\end{array}$} \\
\hline & & Mean & 95th \\
\hline \multicolumn{2}{|c|}{0.1} & $9.98 \mathrm{E}-06$ & $0.00 E+00$ \\
\hline \multicolumn{2}{|c|}{0.2} & 4.40E-04 & 2.35E-03 \\
\hline \multicolumn{2}{|c|}{0.3} & $1.91 \mathrm{E}-03$ & $1.32 \mathrm{E}-02$ \\
\hline \multicolumn{2}{|c|}{0.4} & $4.11 \mathrm{E}-03$ & $2.67 \mathrm{E}-02$ \\
\hline \multicolumn{2}{|c|}{0.5} & $6.42 \mathrm{E}-03$ & $2.85 \mathrm{E}-02$ \\
\hline \multicolumn{2}{|c|}{0.6} & 8.59E-03 & $3.16 \mathrm{E}-02$ \\
\hline \multicolumn{2}{|c|}{0.7} & 1.07E-02 & 3.33E-02 \\
\hline \multicolumn{2}{|c|}{0.8} & 1.31E-02 & $4.12 \mathrm{E}-02$ \\
\hline \multicolumn{2}{|c|}{0.9} & $1.56 \mathrm{E}-02$ & 4.13E-02 \\
\hline \multicolumn{2}{|c|}{1.0} & $1.81 \mathrm{E}-02$ & $4.48 \mathrm{E}-02$ \\
\hline
\end{tabular}


Table 4.5.8 Mean Early Fatalities for I-131 Release (10 People/Sq. Mi.): Case 1 Release Timing

Inventory: 3800 MWth PWR

I-131 Only

Release Height: 82 Ft.

Energy: 0 MW

Weather: 80th Percentile

Population: 10 People/Sq. Mi.

Calculation: Early Fatalities

\begin{tabular}{|c|c|c|c|c|}
\hline & \multicolumn{2}{|c|}{ Time of Release } & Duration & Alarm Time \\
\hline Case 1 & \multicolumn{2}{|c|}{$2 \mathrm{Hrs}$} & $1 \mathrm{Hr}$ & $2 \mathrm{Hrs}$ \\
\hline \multirow{2}{*}{\multicolumn{2}{|c|}{$\begin{array}{l}\text { I-131 Release } \\
\text { Fraction }\end{array}$}} & \multicolumn{3}{|c|}{$\begin{array}{c}\text { Early Fatalities } \\
(0.4-1.4 \mathrm{Mi})\end{array}$} \\
\hline & & & Iean & 95th \\
\hline \multicolumn{2}{|c|}{0.2} & & 4E-04 & $2.68 \mathrm{E}-03$ \\
\hline \multicolumn{2}{|c|}{0.3} & & $8 E-03$ & $2.68 \mathrm{E}-02$ \\
\hline \multicolumn{2}{|c|}{0.4} & & $5 \mathrm{E}-02$ & $6.74 \mathrm{E}-02$ \\
\hline \multicolumn{2}{|c|}{0.5} & & $1 E-02$ & $1.71 E-01$ \\
\hline \multicolumn{2}{|c|}{0.6} & & 10E-02 & 2.73E-01 \\
\hline \multicolumn{2}{|c|}{0.8} & & $4 E-02$ & $6.45 \mathrm{E}-01$ \\
\hline \multicolumn{2}{|c|}{1.0} & & $10 E-01$ & $9.18 \mathrm{E}-01$ \\
\hline
\end{tabular}


4 Results

Table 4.5.9 Mean Early Fatalities for I-131 Release (10 People/Sq. Mi.): Case 2 Release Timing Inventory: 3800 MWth PWR

$$
\text { I-131 Only }
$$

Release Height: $82 \mathrm{Ft}$.

Energy: 0 MW

Weather: 80th Percentile

Population: 10 People/Sq. Mi.

Calculation: Early Fatalities

\begin{tabular}{|c|c|c|c|}
\hline & Time of Release & Duration & Alarm Time \\
\hline Case 2 & $4 \mathrm{Hrs}$ & $2 \mathrm{Hrs}$ & $4 \mathrm{Hrs}$ \\
\hline \multirow{2}{*}{\multicolumn{2}{|c|}{$\begin{array}{l}\text { 1-131 Release } \\
\text { Fraction }\end{array}$}} & \multicolumn{2}{|c|}{$\begin{array}{c}\text { Early Fatalities } \\
(0.4-1.4 \mathrm{Mi})\end{array}$} \\
\hline & & Mean & 95th \\
\hline \multicolumn{2}{|c|}{0.2} & $1.74 \mathrm{E}-04$ & $5.93 E-04$ \\
\hline \multicolumn{2}{|c|}{0.3} & $1.53 E-03$ & $6.40 \mathrm{E}-03$ \\
\hline \multicolumn{2}{|c|}{0.4} & $5.36 E-03$ & $3.60 \mathrm{E}-02$ \\
\hline \multicolumn{2}{|c|}{0.5} & $1.21 \mathrm{E}-02$ & $7.96 \mathrm{E}-02$ \\
\hline \multicolumn{2}{|c|}{0.6} & $2.28 \mathrm{E}-02$ & $1.32 \mathrm{E}-01$ \\
\hline \multicolumn{2}{|c|}{0.8} & $5.60 \mathrm{E}-02$ & 3.31E-01 \\
\hline \multicolumn{2}{|c|}{1.0} & $1.04 \mathrm{E}-01$ & $5.48 E-01$ \\
\hline
\end{tabular}


Table 4.5.10 Modified PWR Source Terms

Use $100 \%$ of nobles and adjust the fractions of other isotopes to result in an estimated one early fatality with a density of 1000 people/sq. mi. Use both Cases 1 and 2.

\begin{tabular}{|c|c|c|c|c|c|c|c|c|}
\hline NG & $\mathbf{I}$ & Cs & Te & Sr & $\mathbf{R u}$ & $\mathbf{L a}$ & $\mathrm{Ce}$ & $\mathbf{B a}$ \\
\hline 1.0 & .0021 & .0021 & .0012 & .00035 & .000053 & .000053 & .000053 & .00035 \\
\hline \multicolumn{9}{|c|}{ Mean Early Fatalities (0.4-1.4 Mi): 1.04} \\
\hline \multicolumn{9}{|c|}{ MPWR2: 4 Hour Release Time, 2 Hour Duration } \\
\hline NG & $\mathbf{I}$ & Cs & Te & Sr & $\mathbf{R u}$ & $\mathbf{L a}$ & $\mathrm{Ce}$ & $\mathbf{B a}$ \\
\hline 1.0 & 0.018 & .018 & 0.011 & .0031 & .00046 & .00046 & .00046 & .0031 \\
\hline \multicolumn{9}{|c|}{ Mean Early Fatalities (0.4-1.4 Mi): 0.997} \\
\hline \multicolumn{9}{|c|}{ MPWR3: 12 Hour Release Time, 1 Hour Duration } \\
\hline NG & $\mathbf{I}$ & Cs & Te & Sr & $\mathbf{R u}$ & $\mathbf{L a}$ & $\mathrm{Ce}$ & $\mathbf{B a}$ \\
\hline 1.0 & .028 & .028 & .017 & .0047 & .00071 & .00071 & .00071 & .0047 \\
\hline
\end{tabular}

Table 4.5.11 Estimated Mean Number of Individuals Exceeding Threshold Dose for the Modified PWR for Source Terms MPWR1, MPWR2 and MPWR3 as Defined in Table 4.5.10.

\begin{tabular}{|l|c|c|c|c|}
\hline \multicolumn{1}{|c|}{ Organ } & $\begin{array}{c}\text { Early Acute } \\
\text { Threshold } \\
\text { Dose }\end{array}$ & MPWR1 & MPWR2 & MPWR3 \\
\hline Red Marrow & 150 Rem & 16.4 & 17.5 & 11.1 \\
\hline Lungs & 500 Rem & 0.113 & 1.48 & 2.46 \\
\hline Lower Large Intestine & 800 Rem & 0 & 0.0007 & 0.113 \\
\hline
\end{tabular}


Table 4.5.12 Released Inventory and Fractional Release for the Three Modified PWR Source Terms With Sheltering

\begin{tabular}{|c|c|c|c|c|c|c|c|c|}
\hline Isotope & Group & $\begin{array}{c}\text { Core } \\
\text { Inventory } \\
(\mathbf{C i})\end{array}$ & $\begin{array}{c}\text { MPWR1 } \\
\text { (Ci) }\end{array}$ & $\begin{array}{l}\text { MPWR1 } \\
\text { Fraction }\end{array}$ & $\begin{array}{c}\text { MPWR2 } \\
\text { (Ci) }\end{array}$ & $\begin{array}{l}\text { MPWR2 } \\
\text { Fraction }\end{array}$ & $\begin{array}{c}\text { MPWR3 } \\
\text { (Ci) }\end{array}$ & $\begin{array}{l}\text { MPWR3 } \\
\text { Fraction }\end{array}$ \\
\hline CO-58 & 6 & $9.7 E+05$ & $5.0 \mathrm{E}+01$ & 5.2E-05 & $4.3 E+02$ & 4.5E-04 & $6.7 E+02$ & $6.9 \mathrm{E}-04$ \\
\hline CO-60 & 6 & $7.4 \mathrm{E}+05$ & $3.8 \mathrm{E}+01$ & 5.2E-05 & $3.3 E+02$ & 4.5E-04 & $5.1 E+02$ & $6.9 \mathrm{E}-04$ \\
\hline KR-85 & 1 & $7.5 \mathrm{E}+05$ & $7.3 E+05$ & 9.7E-01 & $7.3 E+05$ & 9.7E-01 & $7.3 \mathrm{E}+05$ & $9.7 \mathrm{E}-01$ \\
\hline KR-85M & 1 & $3.5 \mathrm{E}+07$ & $2.5 E+07$ & 7.1E-01 & $1.8 \mathrm{E}+07$ & $5.2 \mathrm{E}-01$ & $5.3 \mathrm{E}+06$ & $1.5 \mathrm{E}-01$ \\
\hline KR-87 & 1 & $6.4 \mathrm{E}+07$ & $2.1 \mathrm{E}+07$ & $3.3 \mathrm{E}-01$ & $6.9 \mathrm{E}+06$ & $1.1 \mathrm{E}-01$ & $8.7 \mathrm{E}+04$ & $1.4 \mathrm{E}-03$ \\
\hline KR-88 & 1 & $8.6 \mathrm{E}+07$ & $5.1 \mathrm{E}+07$ & $5.9 \mathrm{E}-01$ & $3.1 \mathrm{E}+07$ & $3.6 \mathrm{E}-01$ & $4.3 E+06$ & 5.0E-02 \\
\hline RB-86 & 3 & $5.7 \mathrm{E}+04$ & $1.2 \mathrm{E}+02$ & $2.0 \mathrm{E}-03$ & $9.9 \mathrm{E}+02$ & $1.7 \mathrm{E}-02$ & $1.5 \mathrm{E}+03$ & 2.7E-02 \\
\hline SR-89 & 5 & $1.1 \mathrm{E}+08$ & 3.7E+04 & 3.4E-04 & $3.3 E+05$ & $3.0 \mathrm{E}-03$ & $4.9 E+05$ & 4.6E-03 \\
\hline SR-90 & 5 & $5.8 \mathrm{E}+06$ & $2.0 \mathrm{E}+03$ & 3.4E-04 & $1.8 \mathrm{E}+04$ & $3.0 \mathrm{E}-03$ & $2.7 \mathrm{E}+04$ & 4.6E-03 \\
\hline SR-91 & 5 & $1.4 \mathrm{E}+08$ & $4.1 \mathrm{E}+04$ & $2.9 \mathrm{E}-04$ & $3.1 E+05$ & $2.3 \mathrm{E}-03$ & $2.7 E+05$ & $1.9 \mathrm{E}-03$ \\
\hline SR-92 & 5 & $1.4 \mathrm{E}+08$ & $2.9 E+04$ & $2.0 \mathrm{E}-04$ & $1.6 \mathrm{E}+05$ & 1.1E-03 & $3.1 \mathrm{E}+04$ & 2.1E-04 \\
\hline$Y-90$ & 7 & $6.3 \mathrm{E}+06$ & $3.6 \mathrm{E}+02$ & 5.7E-05 & $3.4 \mathrm{E}+03$ & 5.5E-04 & $7.1 \mathrm{E}+03$ & 1.1E-03 \\
\hline$Y-91$ & 7 & $1.3 E+08$ & $6.8 E+03$ & $5.2 \mathrm{E}-05$ & $5.9 E+04$ & $4.5 \mathrm{E}-04$ & $9.3 E+04$ & 7.1E-04 \\
\hline $\mathrm{Y}-92$ & 7 & $1.5 E+08$ & $1.7 \mathrm{E}+04$ & $1.2 \mathrm{E}-04$ & 1.7E +05 & 1.2 & $1.2 E+05$ & $7.9 \mathrm{E}-04$ \\
\hline$Y-93$ & 7 & $1.6 \mathrm{E}+08$ & $7.4 \mathrm{E}+03$ & $4.5 \mathrm{E}-05$ & $5.6 \mathrm{E}+04$ & 3.4E-04 & $5.0 \mathrm{E}+04$ & 3.0E-04 \\
\hline ZR-95 & 7 & $1.7 \mathrm{E}+08$ & $8.6 \mathrm{E}+03$ & $5.2 \mathrm{E}-05$ & $7.4 \mathrm{E}+04$ & 4.5E-04 & $1.1 \mathrm{E}+05$ & $6.9 \mathrm{E}-04$ \\
\hline ZR-97 & 7 & $1.7 E+08$ & 8.2E +03 & $4.8 \mathrm{E}-05$ & $6.6 E+04$ & $3.8 \mathrm{E}-04$ & $7.3 E+04$ & 4.2E- 04 \\
\hline NB-95 & 7 & $1.6 \mathrm{E}+08$ & $8.1 E+03$ & $5.2 \mathrm{E}-05$ & $7.1 E+04$ & $4.5 E-04$ & $1.1 \mathrm{E}+05$ & $6.9 \mathrm{E}-04$ \\
\hline MO-99 & 6 & $1.8 E+08$ & $9.3 E+03$ & 5.1E-05 & $7.9 E+04$ & $4.3 E-04$ & $1.1 \mathrm{E}+05$ & 6.1E-04 \\
\hline TC-99M & 6 & $1.6 \mathrm{E}+08$ & 8.4E+03 & 5.3E-05 & $7.4 \mathrm{E}+04$ & 4.7E-04 & $1.2 \mathrm{E}+05$ & 7.3E-04 \\
\hline RU-103 & 6 & $1.4 E+08$ & $7.1 \mathrm{E}+03$ & $5.2 \mathrm{E}-05$ & $6.1 E+04$ & 4.5E-04 & $9.4 \mathrm{E}+04$ & $6.9 \mathrm{E}-04$ \\
\hline RU-105 & 6 & $8.9 E+07$ & $3.4 \mathrm{E}+03$ & 3.8E-05 & $2.1 E+04$ & $2.4 \mathrm{E}-04$ & $9.4 E+03$ & $1.1 \mathrm{E}-04$ \\
\hline RU-106 & 6 & $3.1 \mathrm{E}+07$ & $1.6 E+03$ & $5.2 \mathrm{E}-05$ & $1.4 \mathrm{E}+04$ & 4.5E-04 & $2.1 E+04$ & $6.9 \mathrm{E}-04$ \\
\hline RH-105 & 6 & $6.2 \mathrm{E}+07$ & $3.2 \mathrm{E}+03$ & $5.2 \mathrm{E}-05$ & $2.8 \mathrm{E}+04$ & $4.5 E-04$ & $3.9 E+04$ & $6.4 \mathrm{E}-04$ \\
\hline SB-127 & 4 & $8.4 E+06$ & $9.7 \mathrm{E}+03$ & $1.2 \mathrm{E}-03$ & 8.7E+04 & $1.0 \mathrm{E}-02$ & $1.3 E+05$ & 1.5E-02 \\
\hline SB-129 & 4 & $3.0 \mathrm{E}+07$ & $2.5 \mathrm{E}+04$ & 8.5E-04 & $1.7 \mathrm{E}+05$ & $5.7 \mathrm{E}-03$ & $7.2 \mathrm{E}+04$ & $2.4 \mathrm{E}-03$ \\
\hline TE-127 & 4 & $8.1 E+06$ & $9.5 \mathrm{E}+03$ & $1.2 \mathrm{E}-03$ & 8.7E+04 & $1.1 \mathrm{E}-02$ & $1.3 \mathrm{E}+05$ & $1.6 \mathrm{E}-02$ \\
\hline TE-127M & 4 & $1.1 \mathrm{E}+06$ & $1.3 E+03$ & $1.2 \mathrm{E}-03$ & $1.1 \mathrm{E}+04$ & 1.1E-02 & $1.8 \mathrm{E}+04$ & 1.7E-02 \\
\hline TE-129 & 4 & $2.8 E+07$ & $3.0 \mathrm{E}+04$ & $1.1 \mathrm{E}-03$ & $2.2 \mathrm{E}+05$ & 7.8E-03 & $9.9 E+04$ & $3.5 \mathrm{E}-03$ \\
\hline
\end{tabular}


Table 4.5.12 (continued)

\begin{tabular}{|c|c|c|c|c|c|c|c|c|}
\hline Isotope & Group & $\begin{array}{c}\text { Core } \\
\text { Inventory } \\
\text { (Ci) }\end{array}$ & $\begin{array}{c}\text { MPWR1 } \\
\text { (Ci) }\end{array}$ & $\begin{array}{l}\text { MPWR1 } \\
\text { Fraction }\end{array}$ & $\begin{array}{c}\text { MPWR2 } \\
\text { (Ci) }\end{array}$ & $\begin{array}{l}\text { MPWR2 } \\
\text { Fraction }\end{array}$ & $\begin{array}{c}\text { MPWR3 } \\
\text { (Ci) }\end{array}$ & $\begin{array}{l}\text { MPWR3 } \\
\text { Fraction }\end{array}$ \\
\hline TE-129M & 4 & $7.4 \mathrm{E}+06$ & $8.6 E+03$ & $1.2 \mathrm{E}-03$ & $7.8 \mathrm{E}+04$ & $1.1 \mathrm{E}-02$ & $1.2 \mathrm{E}+05$ & $1.6 \mathrm{E}-02$ \\
\hline TE-131M & 4 & $1.4 \mathrm{E}+07$ & $1.6 \mathrm{E}+04$ & $1.1 E-03$ & $1.4 \mathrm{E}+05$ & 9.8E-03 & $1.8 \mathrm{E}+05$ & $1.3 \mathrm{E}-02$ \\
\hline TE-132 & 4 & $1.4 E+08$ & $1.6 \mathrm{E}+05$ & $1.1 \mathrm{E}-03$ & $1.5 \mathrm{E}+06$ & 1.0E-02 & $2.1 E+06$ & $1.5 \mathrm{E}-02$ \\
\hline I-131 & 2 & $9.7 \mathrm{E}+07$ & $2.0 \mathrm{E}+05$ & $2.0 \mathrm{E}-03$ & $1.7 \mathrm{E}+06$ & $1.7 \mathrm{E}-02$ & $2.5 E+06$ & $2.6 \mathrm{E}-02$ \\
\hline $\mathrm{I}-132$ & 2 & $1.4 E+08$ & $2.3 E+05$ & $1.6 \mathrm{E}-03$ & $1.8 E+06$ & $1.2 E-02$ & $2.2 E+06$ & $1.5 \mathrm{E}-02$ \\
\hline I-133 & 2 & $2.0 \mathrm{E}+08$ & $3.9 \mathrm{E}+05$ & $1.9 \mathrm{E}-03$ & $3.1 E+06$ & $1.5 \mathrm{E}-02$ & $3.7 E+06$ & $1.8 \mathrm{E}-02$ \\
\hline I-134 & 2 & $2.2 \mathrm{E}+08$ & $9.4 \mathrm{E}+04$ & 4.2E-04 & $1.7 \mathrm{E}+05$ & 7.4E-04 & $4.6 \mathrm{E}+02$ & 2.1E-06 \\
\hline I-135 & 2 & $1.9 \mathrm{E}+08$ & $3.2 \mathrm{E}+05$ & $1.7 \mathrm{E}-03$ & $2.2 E+06$ & 1.1E-02 & $1.5 E+06$ & 7.7E-03 \\
\hline XE-133 & 1 & $2.0 \mathrm{E}+08$ & $2.0 \mathrm{E}+08$ & $9.6 \mathrm{E}-01$ & $1.9 E+08$ & 9.5E-01 & $1.9 E+08$ & 9.1E-01 \\
\hline XE-135 & 1 & $3.8 \mathrm{E}+07$ & $3.2 \mathrm{E}+07$ & $8.4 \mathrm{E}-01$ & $2.8 E+07$ & 7.4E-01 & $1.7 E+07$ & 4.4E-01 \\
\hline CS-134 & 3 & $1.3 \mathrm{E}+07$ & $2.7 \mathrm{E}+04$ & $2.0 \mathrm{E}-03$ & $2.3 \mathrm{E}+05$ & $1.8 \mathrm{E}-02$ & $3.6 \mathrm{E}+05$ & $E-02$ \\
\hline CS-136 & 3 & $4.0 \mathrm{E}+06$ & $8.1 \mathrm{E}+03$ & $2.0 \mathrm{E}-03$ & $6.9 E+04$ & $1.7 \mathrm{E}-02$ & $1.1 \mathrm{E}+05$ & 2.7E-02 \\
\hline CS-137 & 3 & $7.3 \mathrm{E}+06$ & $1.5 \mathrm{E}+04$ & $2.0 \mathrm{E}-03$ & $1.3 \mathrm{E}+05$ & $1.8 \mathrm{E}-02$ & $2.0 \mathrm{E}+05$ & 2.7E-02 \\
\hline BA-139 & 9 & $1.9 \mathrm{E}+08$ & $2.4 \mathrm{E}+04$ & $1.3 \mathrm{E}-04$ & $7.7 E+04$ & 4.1E-04 & $2.1 \mathrm{E}+03$ & $1.1 \mathrm{E}-05$ \\
\hline BA-140 & 9 & $1.9 \mathrm{E}+08$ & $6.3 E+04$ & $3.4 \mathrm{E}-04$ & $5.6 \mathrm{E}+05$ & 3.0E-03 & $8.3 E+05$ & 4.5E-03 \\
\hline LA-140 & 7 & $1.9 \mathrm{E}+08$ & $1.2 E+04$ & $6.1 E-05$ & $1.2 \mathrm{E}+05$ & $6.1 E-04$ & $2.7 E+05$ & $1.4 \mathrm{E}-03$ \\
\hline LA-141 & 7 & $1.8 \mathrm{E}+08$ & $6.4 \mathrm{E}+03$ & $3.6 \mathrm{E}-05$ & $3.9 \mathrm{E}+04$ & $2.2 \mathrm{E}-04$ & $1.5 \mathrm{E}+04$ & 8.4E-05 \\
\hline LA-142 & 7 & $1.7 E+08$ & 3.7E+03 & 2.2E-05 & $1.3 \mathrm{E}+04$ & $7.8 \mathrm{E}-05$ & $6.2 E+02$ & 3.7E-06 \\
\hline CE-141 & 8 & $1.7 \mathrm{E}+08$ & $8.8 E+03$ & $5.2 \mathrm{E}-05$ & $7.6 \mathrm{E}+04$ & $4.5 \mathrm{E}-04$ & $1.2 \mathrm{E}+05$ & $6.9 \mathrm{E}-04$ \\
\hline CE-143 & 8 & $1.7 E+08$ & $8.2 E+03$ & 4.9E-05 & $6.8 \mathrm{E}+04$ & 4.1E-04 & $8.9 E+04$ & 5.4E-04 \\
\hline CE-144 & 8 & $1.0 \mathrm{E}+08$ & $5.3 E+03$ & 5.2E-05 & $4.6 \mathrm{E}+04$ & $4.5 \mathrm{E}-04$ & $7.1 \mathrm{E}+04$ & 6.9E-04 \\
\hline PR-143 & 7 & $1.6 \mathrm{E}+08$ & $8.4 E+03$ & $5.2 \mathrm{E}-05$ & $7.3 E+04$ & $4.5 \mathrm{E}-04$ & $1.1 \mathrm{E}+05$ & 6.9E-04 \\
\hline ND-147 & 7 & $7.3 E+07$ & $3.7 \mathrm{E}+03$ & $\mathrm{E}-05$ & $3.2 \mathrm{E}+04$ & 4.4E-04 & $4.9 E+04$ & 6.7E-04 \\
\hline NP-239 & 8 & $1.9 \mathrm{E}+09$ & $9.8 \mathrm{E}+04$ & 5.0E-05 & $8.3 E+05$ & 4.3E-04 & $1.2 \mathrm{E}+06$ & 6.0E-04 \\
\hline PU-238 & 8 & $1.1 \mathrm{E}+05$ & $5.7 \mathrm{E}+\infty 0$ & $5.2 \mathrm{E}-05$ & $4.9 \mathrm{E}+01$ & 4.5E-04 & $7.6 \mathrm{E}+01$ & $6.9 \mathrm{E}-04$ \\
\hline PU-239 & 8 & $2.5 \mathrm{E}+04$ & $1.3 \mathrm{E}+\infty 0$ & $5.2 \mathrm{E}-05$ & $1.1 \mathrm{E}+01$ & $4.5 \mathrm{E}-04$ & $1.7 \mathrm{E}+01$ & $6.9 \mathrm{E}-04$ \\
\hline PU-240 & 8 & 3.1E + 04 & $1.6 \mathrm{E}+\infty 0$ & $5.2 \mathrm{E}-05$ & $1.4 \mathrm{E}+01$ & $4.5 \mathrm{E}-04$ & $2.2 \mathrm{E}+01$ & $6.9 \mathrm{E}-04$ \\
\hline PU-241 & 8 & $5.3 E+06$ & $2.7 \mathrm{E}+02$ & 5.2E-05 & $2.4 \mathrm{E}+03$ & 4.5E-04 & $3.7 \mathrm{E}+03$ & $6.9 \mathrm{E}-04$ \\
\hline AM-241 & 7 & $3.5 \mathrm{E}+03$ & $1.8 \mathrm{E}-01$ & $5.2 \mathrm{E}-05$ & $1.6 \mathrm{E}+00$ & 4.5E-04 & $2.4 \mathrm{E}+00$ & $6.9 \mathrm{E}-04$ \\
\hline CM-242 & 7 & $1.3 E+06$ & $6.9 \mathrm{E}+01$ & $5.2 \mathrm{E}-05$ & $6.0 \mathrm{E}+02$ & 4.5E-04 & $9.2 \mathrm{E}+02$ & $6.9 \mathrm{E}-04$ \\
\hline CM-244 & 7 & $.7 .8 \mathrm{E}+04$ & $4.0 \mathrm{E}+\infty 0$ & 5.2E-05 & $3.5 \mathrm{E}+01$ & 4.5E-04 & $5.4 \mathrm{E}+01$ & $6.9 \mathrm{E}-04$ \\
\hline
\end{tabular}



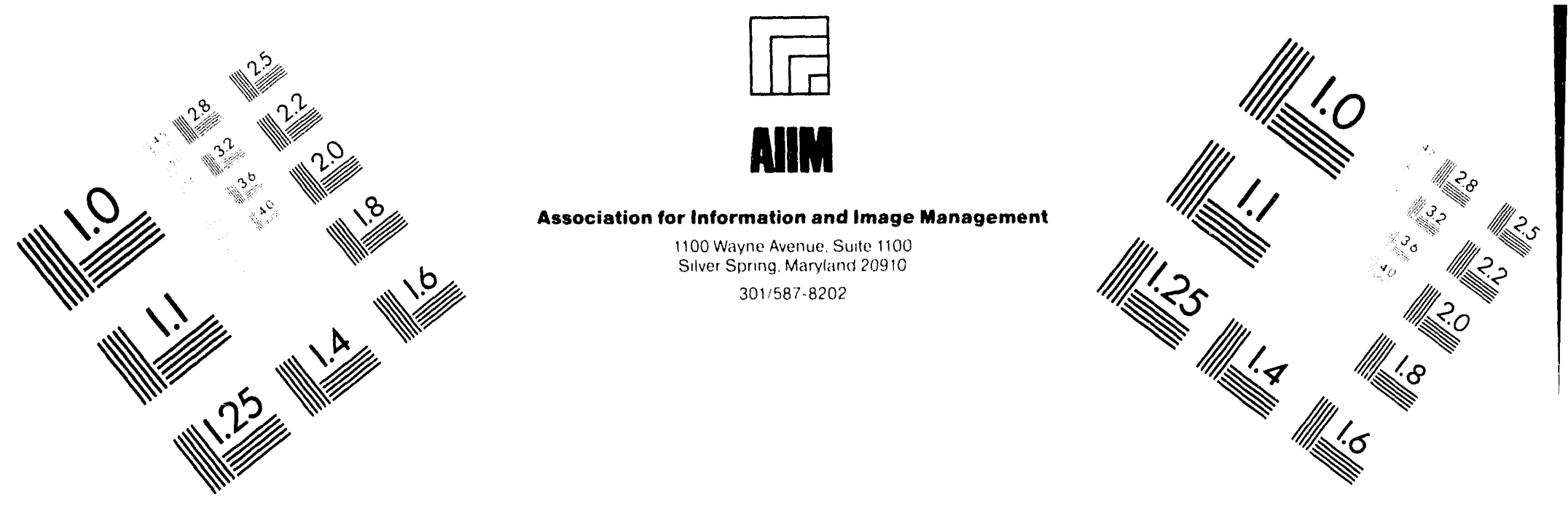

\section{Centimeter}

| Inches
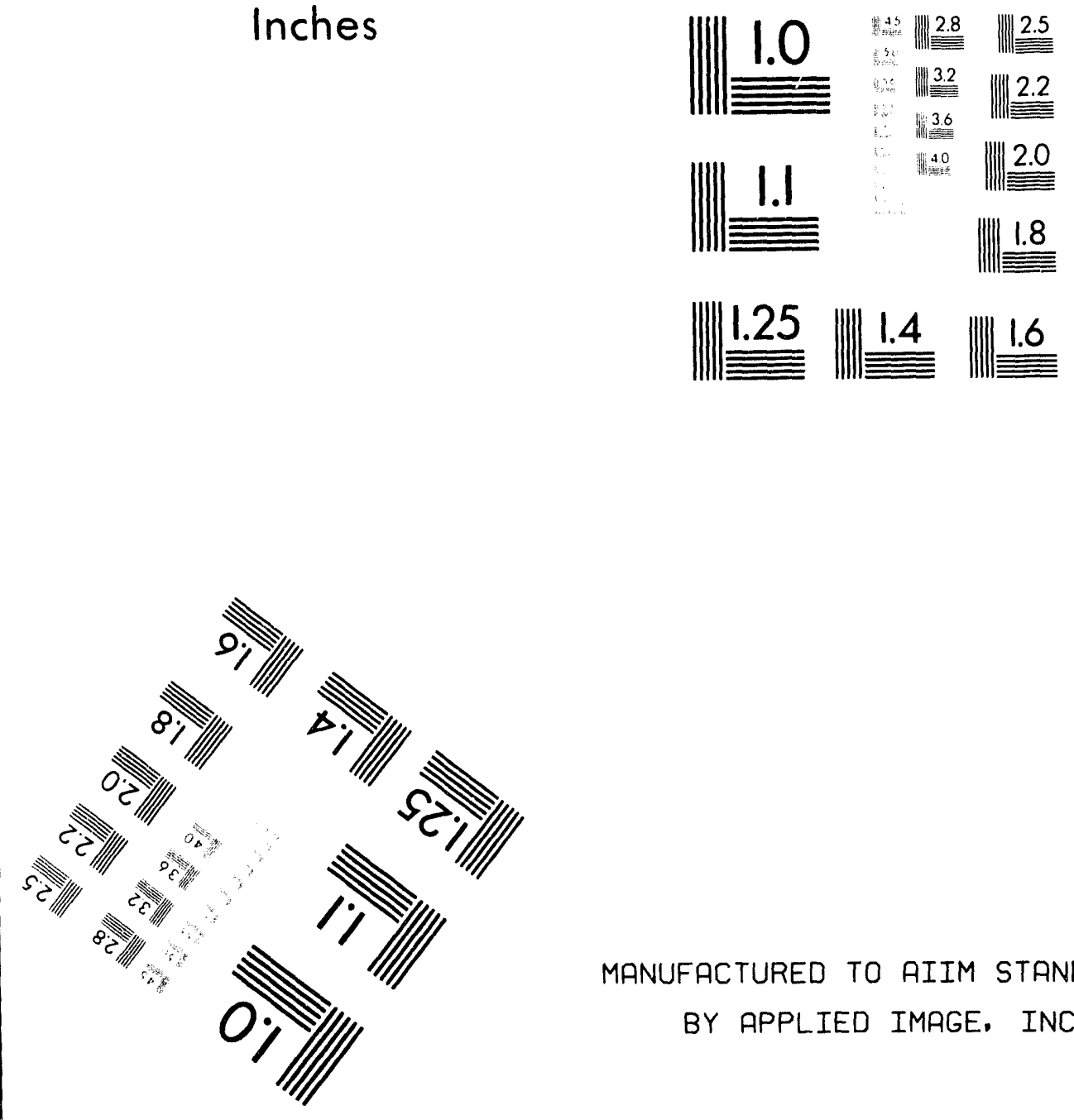

MANUFACTURED TO AIIM STANDARDS

BY APPLIED IMAGE. INC.

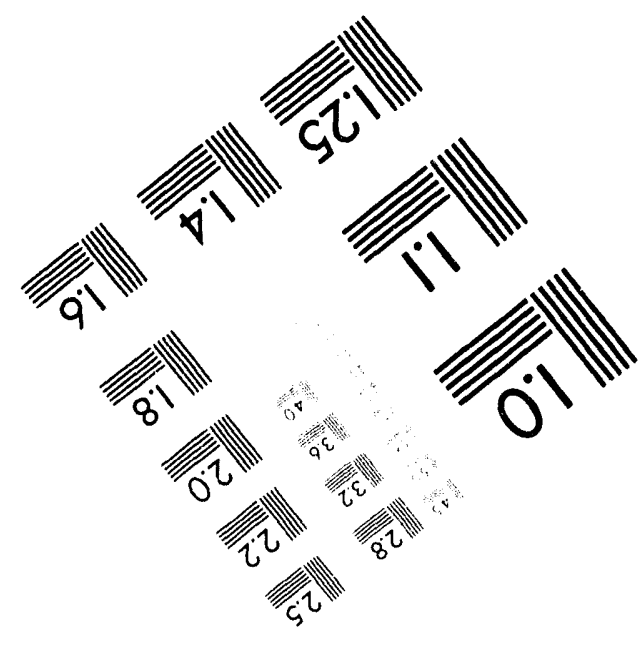



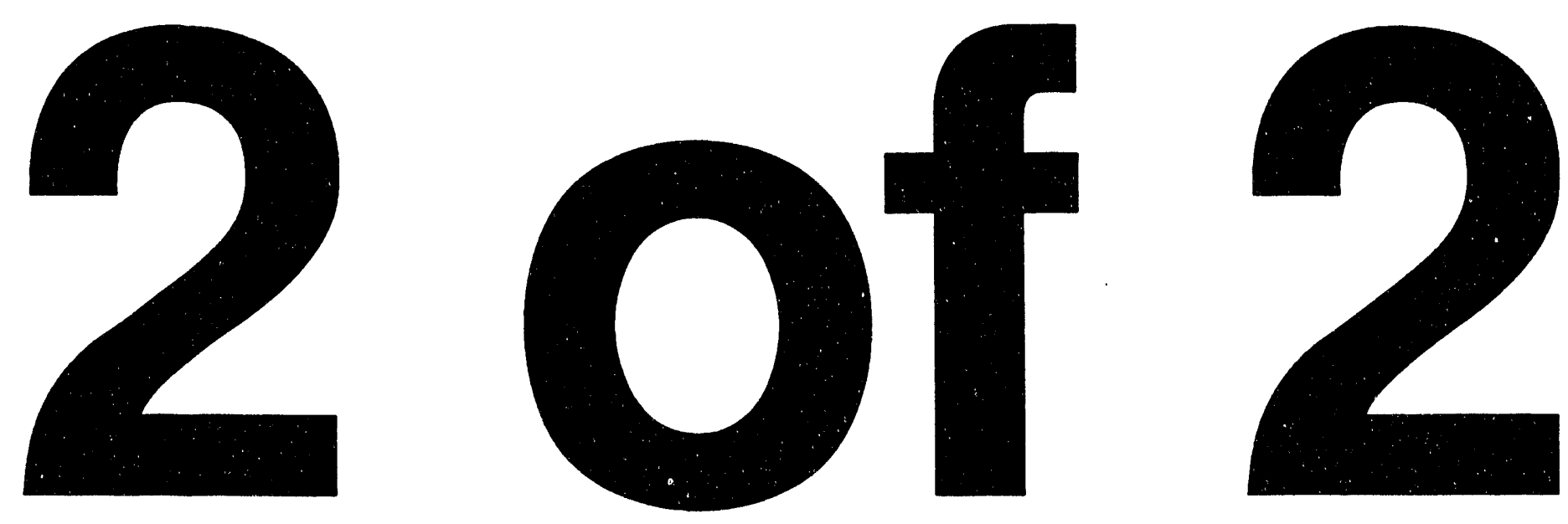
Table 4.5.13 Grouped Release Inventory and Fractional Group Releases for the Three PWR Source Terms with Sheltering

\begin{tabular}{|c|c|c|c|c|c|c|c||}
\hline Group & $\begin{array}{c}\text { Core } \\
\text { Inventory } \\
(\mathrm{Ci})\end{array}$ & $\begin{array}{c}\text { MPRW1 } \\
(\mathrm{Ci})\end{array}$ & $\begin{array}{c}\text { MPRW1 } \\
\text { Fraction }\end{array}$ & $\begin{array}{c}\text { MPWR2 } \\
(\mathrm{Ci})\end{array}$ & $\begin{array}{c}\text { MPWR2 } \\
\text { Fraction }\end{array}$ & $\begin{array}{c}\text { MPWR3 } \\
(\mathrm{Ci})\end{array}$ & $\begin{array}{c}\text { MPWR3 } \\
\text { Fraction }\end{array}$ \\
\hline 1 & $4.3 \mathrm{E}+08$ & $3.3 \mathrm{E}+08$ & $7.6 \mathrm{E}-01$ & $2.8 \mathrm{E}+08$ & $6.5 \mathrm{E}-01$ & $2.1 \mathrm{E}+08$ & $5.0 \mathrm{E}-01$ \\
\hline 2 & $8.6 \mathrm{E}+08$ & $1.2 \mathrm{E}+06$ & $1.4 \mathrm{E}-03$ & $9.0 \mathrm{E}+06$ & $1.0 \mathrm{E}-02$ & $9.9 \mathrm{E}+06$ & $1.2 \mathrm{E}-02$ \\
\hline 3 & $2.4 \mathrm{E}+07$ & $5.0 \mathrm{E}+04$ & $2.0 \mathrm{E}-03$ & $4.3 \mathrm{E}+05$ & $1.8 \mathrm{E}-02$ & $6.6 \mathrm{E}+05$ & $2.7 \mathrm{E}-02$ \\
\hline 4 & $2.4 \mathrm{E}+08$ & $2.6 \mathrm{E}+05$ & $1.1 \mathrm{E}-03$ & $2.2 \mathrm{E}+06$ & $9.4 \mathrm{E}-03$ & $2.8 \mathrm{E}+06$ & $1.2 \mathrm{E}-02$ \\
\hline 5 & $4.0 \mathrm{E}+08$ & $2.1 \mathrm{E}+05$ & $5.2 \mathrm{E}-04$ & $1.1 \mathrm{E}+06$ & $2.8 \mathrm{E}-03$ & $2.2 \mathrm{E}+05$ & $5.5 \mathrm{E}-04$ \\
\hline 6 & $6.6 \mathrm{E}+08$ & $1.5 \mathrm{E}+04$ & $2.3 \mathrm{E}-05$ & $1.2 \mathrm{E}+05$ & $1.9 \mathrm{E}-04$ & $1.6 \mathrm{E}+05$ & $2.5 \mathrm{E}-04$ \\
\hline 7 & $1.7 \mathrm{E}+09$ & $9.1 \mathrm{E}+04$ & $5.3 \mathrm{E}-05$ & $7.7 \mathrm{E}+05$ & $4.5 \mathrm{E}-04$ & $1.0 \mathrm{E}+06$ & $5.8 \mathrm{E}-04$ \\
\hline 8 & $2.4 \mathrm{E}+09$ & $1.2 \mathrm{E}+05$ & $5.0 \mathrm{E}-05$ & $1.0 \mathrm{E}+06$ & $4.3 \mathrm{E}-04$ & $1.4 \mathrm{E}+06$ & $6.0 \mathrm{E}-04$ \\
\hline 9 & $3.8 \mathrm{E}+08$ & $8.7 \mathrm{E}+04$ & $2.3 \mathrm{E}-04$ & $6.4 \mathrm{E}+05$ & $1.7 \mathrm{E}-03$ & $8.4 \mathrm{E}+05$ & $2.2 \mathrm{E}-03$ \\
\hline
\end{tabular}




\section{TECHNICAL FINDINGS}

A number of calculations with different assumptions and inputs have been reported in the previous sections to establish a data base of information which would be useful in attempting to quantify a "large release". Most of the calculations have been based on determining the characteristics of various releases which have the potential to lead to one early fatality it the mean at a representative site with a specified population density and meteorology. At this generic site, a number of emergency responses were assumed based on the assumptions made at the NUREG1150 sites or from information derived from other studies. For each of the emergency response assumptions, a set of calculations were performed with source terms selected from the ones contained in Appendix A. A series of modifications were performed to the selected source terms in each case. These modification included: changes in the timing of the release, scaling of the initial core inventory (through adjustment of the power level), or modifications of the release fractions of selected groups of radionuclides, in order to arrive an estimated one mean early fatality as calculated by MACCS.

The basic characteristics of these adjusted "candidate" large release source terms are shown in Table 5.1. These characteristics include: timing of release, energy and height of release, and the actual amount of curies of six categories of radionuclides released to the environment, noble gases, iodine, cesium, tellurium, barium, and strontium. As discussed above, the modification or scaling was performed on one or more of the original source terms' characteristics: timing of release, release fractions, or the initia! core inventory, for each of the emergency response assumptions. The modified source terms which then have the potential to lead to one mean early fatality are discussed separately below under each of the emergency response assumptions. These calculations indicate that the large release guideline is generally more conservative than the $\mathrm{QHO}$ by one or more orders of magnitude.

\subsection{No Evacuation Case}

Four candidate source terms were explored in this case, MGG3, MLAS3, MLAS6, and MZ2. The first three, in particular MLAS3 and MLAS6, are releases at high energy; the corresponding dilution of which results appears to require large amounts of curies released to the environment with the potential to lead to one early fatality. MZ2 is a low-energy release and can be compared more readily with some of the other cases. In MZ2, $100 \%$ of the nobles and 3\% of the iodine inventory is released fairly late. The releases of all the other radionuclides was negligible. Correcting for the decay, $260 \mathrm{MCi}$ of noble gases, and $12 \mathrm{MCi}$ of iodine are actually released to the environment in this source term which has the potential to lead to one mean early fatality for the no evacuation case.

\subsection{Mean Evacuation Case}

In the mean evacuation case, there were three candidate source terms, MPB1, MPB6, and MSUR2. All of these are late releases and involve $100 \%$ of the noble gases and substantial fractions of the iodine and tellurium inventory; 29\% (I) and 18\% (Te) for MPB1, 34\% (I) and 23\% (Te) for MPB6, and 18\% (I) and 27\% (Te) for MSUR2. These releases are close to or, in some cases, larger than those reported for Chernobyl.

\subsection{Conservative Evacuation Case}

In the conservative evacuation the candidates are MPB2, MPB3, and MSUR2. The original RPB2 source term from which MPB2 has been derived is a fairly energetic release so the amounts actually needed to be released in order 


\section{Technical Findings}

to result in the potential to lead to one mean early fatality would be somewhat higher than for the non-energetic release. MPB2 has mid release timing while MPB3 has an early to mid release timing. Inspection of the curies released shows that $\mathrm{Mit}-2$ releases $100 \%$ of the nobles, $6 \%$ of the iodine and $4 \%$ of Te, MPB3 releases $100 \%$ nobles, $3 \% \mathrm{I}$ and $2 \% \mathrm{Te}$, and MSUR2 releases $100 \%$ nobles, $4 \% \mathrm{I}$ and $6 \% \mathrm{Te}$.

\subsection{Sheltering Case}

The sheltering case is very similar to the conservative evacuation case. The candidate source terms here are essentially the same as in the conservative evacuation case with small differences in their release fractions.: MPB2, MPB3, and MSUR2. Since the timings and energy are the same as in the conservative evacuation case only the release fractions are discussed here. In each of the candidate source terms, $100 \%$ of the nobles is released, followed by $4.5 \% \mathrm{I}$ and $3 \% \mathrm{Te}$ (MPB2), $2.5 \% \mathrm{I}$ and $2 \% \mathrm{Te}$ (MPB3), and $3 \% \mathrm{I}$ and $4.5 \% \mathrm{Te}$ (MSUR2).

\subsection{Summary}

Based on the information in Table 5.1, two findings should be noted regarding the quantification of a "large release" as the release of "that fraction of the core inventory of specified radionuclides which has the potential to lead to one (or more) mean early fatality":

1. At a representative, generic site, there exist several candidate source terms which meet the above specification of a "large release." The characteristics of these candidate source terms are a function of factors such as emergency protective action assumptions, timing of release, release energy, etc. While the effect of the timing of release can be eliminated by expressing the release in terms of "equivalent curies" of a specific isotope such as I-131, the variability due to the other factors still remains.

2. For all of the "large release" candidates considered, the frequency of the large release guideline of $10^{-6}$ per year of reactor operation will, in general, lead to a higher level of conservatism (i.e., impose a more stringent plant performance standard) than the early fatality quantitative health objective by one (or more) order(s) of magnitude. 
Table 5.1 Candidate Large Release Source Terms

\begin{tabular}{|c|c|c|c|c|c|c|c|c|c|}
\hline \multirow{2}{*}{$\begin{array}{c}\text { Modified } \\
\text { Source } \\
\text { Term }\end{array}$} & \multirow{2}{*}{$\begin{array}{c}\text { Time of } \\
\text { Release } \\
\text { (hour) }\end{array}$} & \multirow{2}{*}{$\begin{array}{c}\text { Energy } \\
\text { of } \\
\text { Release } \\
\text { (watts) }\end{array}$} & \multirow{2}{*}{$\begin{array}{c}\text { Emergency } \\
\text { Response } \\
\text { (EP) }\end{array}$} & \multicolumn{6}{|c|}{ Release from Containment (MCi) } \\
\hline & & & & $\mathrm{Ng}$ & $\mathbf{I}$ & Cs & $\mathrm{Te}$ & $\mathrm{Sr}$ & $\mathbf{B a}$ \\
\hline MGG3 & 13.4 & $3.0 \mathrm{E}+07$ & No & 250 & 48 & 0.33 & 1.4 & 0.75 & 0.61 \\
\hline MLAS3 & 4.2 & $4.4 \mathrm{E}+08$ & No & 380 & 75 & 2 & 10 & 7.4 & 6.4 \\
\hline MLAS6 & 7.5 & $1.0 \mathrm{E}+08$ & No & 180 & 32 & 2.2 & 11 & 7.8 & 6.1 \\
\hline MZ2 & 6.2 & $2.0 \mathrm{E}+05$ & No & 260 & 12 & $<.1$ & 0.1 & $<.1$ & $<.1$ \\
\hline MPB1 & 11.5 & $6.4 \mathrm{E}+07$ & Mean & 250 & 100 & 7.3 & 31 & 18 & 14 \\
\hline MPB6 & 11.3 & $9.6 \mathrm{E}+06$ & Mean & 190 & 120 & 8.3 & 37 & 21 & 17 \\
\hline MSUR2 & 12 & $0.0 \mathrm{E}+00$ & Mean & 230 & 77 & 2 & 41 & 1.4 & 1.5 \\
\hline MPB2 & 7.3 & $6.4 \mathrm{E}+07$ & Cons & 260 & 28 & 1.8 & 8 & 5.1 & 5.9 \\
\hline MPB3 & 4.8 & $9.6 \mathrm{E}+06$ & Cons & 280 & 19 & 1.2 & 6.1 & 4.4 & 3.9 \\
\hline MSUR2 & 12 & $0.0 \mathrm{E}+\infty$ & Cons & 220 & 29 & 0.7 & 15 & 0.53 & 0.55 \\
\hline MPB2 & 7.3 & $6.4 \mathrm{E}+07$ & Shelter & 260 & 22 & 1.3 & 6 & 8.7 & 5.9 \\
\hline MPB3 & 4.8 & $0.0 \mathrm{E}+00$ & Shelter & 280 & 12 & 0.77 & 3.9 & 4.4 & 3.9 \\
\hline MSUR2 & 12 & $0.0 \mathrm{E}+00$ & Shelter & 220 & 14 & 0.36 & 7.7 & 0.53 & 0.55 \\
\hline
\end{tabular}


5 Technical Findings 


\section{REFERENCES}

1. Federal Register, Vol. 51, No. 162, P30028, August 21, 1986.

2. U. S. Nuclear Regulatory Commission, SECY 89-102, May 1989.

3. R. E. Davis, A. H. Hanson and V. Mubayi, "Reassessment of Factors Affecting Siting of Nuclear Power Plants," Draft Letter Report, May 29, 1992 (available in the Public Document Room). The simplified source terms are based on the work reported in: H. P. Nourbaksh, "Estimate of Radionuclide Release Characteristics Into Containment Under Severe Accident Conditions," NUREG/CR-5747, January 1992.

4. U. S. Nuclear Regulatory Commission, "Severe Accident Risks: An Assessment of five U. S. Nuclear Power Plants, "NUREG-1150, 1990.

5. T. D. Brown, A. C. Payne, Jr., L. A. Miller, J. D. Johnson, D. I. Chanin, A. W. Shiver, S. J. Higgins, T. T. Sype, "Integrated Risk Assessment for La Salle Unit 2 Nuclear Power Plant," NUREG/CR-5305, 1992.

6. H. N. Jow, J. L. Sprung, J. A. Rollstin, L. T. Ritchie and D. I. Chanin, "MELCOR Accident Consequence Code System (MACCS)," Volumes I, II and III, NUREG/CR-4691, 1990.

7. J. M. Hans, Jr., and T. C. Sell, “Evacuation Risks: An Evaluation," U. S. Environment Protection Agency, June 1974.

8. Regulatory Guide 4.7, "General Site Suitability Criteria for Nuclear Power Stations, Revision 1, " U. S. Nuclear Regulatory Commission, November 1985. 


\section{APPENDIX A - SIMPLIFIED SOURCE TERMS}


Table A.1 Characteristics of Surry Release Categories

\begin{tabular}{||c|c|c|c|c|c|c|c|c||}
\hline \multirow{2}{*}{$\begin{array}{c}\text { Release } \\
\text { Category }\end{array}$} & $\begin{array}{c}\text { Plant } \\
\text { Damage } \\
\text { State }\end{array}$ & \multicolumn{7}{|c|}{ Accident Progression Characteristics } \\
\cline { 3 - 10 } & $\begin{array}{c}\text { Containment } \\
\text { Failure Time }\end{array}$ & $\begin{array}{c}\text { Containment } \\
\text { Failure Size }\end{array}$ & CCI & Amt CCI & RCS Pres. & VB Mode & Sprays \\
\hline RSUR1 & LOSP & CF at VB & Rupture & Prm Dry & Medium & Lo Pr & Alpha & No \\
\hline RSUR2 & LOSP & CF after CCI & Leak & Prm Dry & Large & Lo Pr & Pour & No \\
\hline RSUR3 & LOSP & No CF & No CF & Prm Dry & Large & Lo Pr & Pour & L + VL \\
\hline RSUR4 & Bypass (V) & No CF & Bypass & Prm Dry & Large & Lo Pr & Pour & No \\
\hline
\end{tabular}

Table A.2 Characteristics of Sequoyah Release Categories

\begin{tabular}{|c|c|c|c|c|c|c|c|c|c|}
\hline \multirow{2}{*}{$\begin{array}{l}\text { Release } \\
\text { Category }\end{array}$} & \multirow{2}{*}{$\begin{array}{c}\text { Plant } \\
\text { Damage } \\
\text { State }\end{array}$} & \multicolumn{8}{|c|}{ Accident Progression Characteristics } \\
\hline & & $\begin{array}{l}\text { Containment } \\
\text { Failure Time }\end{array}$ & $\begin{array}{l}\text { Containment } \\
\text { Failure Size }\end{array}$ & $\mathrm{CCI}$ & Amt CCI & RCS Pres. & VB Mode & ARF & Sprays \\
\hline RSEQI & LOSP & $\begin{array}{c}\text { Early CF } \\
\text { (during CD) }\end{array}$ & Cat. Rupture & Prm Dry & Large & Low & Pour & No & No \\
\hline RSEQ2 & LOSP & $\mathrm{CF}$ at $\mathrm{VB}$ & Cat. Ruplure & Prm Dry & Large & Low & Pour & No & No \\
\hline RSEQ3 & LOCA & Very Late & Rupture & Prm Dp & Large & Low & Porir & $\mathrm{E}+\mathrm{L}$ & No \\
\hline RSEQ4 & LOCA & No CF & No $\mathrm{CF}$ & Prm Dp & Large & Low & Pour & $E+L$ & No \\
\hline RSEQ5 & Bypass & No $C F$ & Bypass & Prm Dry & Large & Low & Pour & $E+L$ & No \\
\hline
\end{tabular}


Table A.3 Characteristics of Zion Release Categories

\begin{tabular}{|c|c|c|c|c|c|c|c|c|}
\hline \multirow{2}{*}{$\begin{array}{l}\text { Release } \\
\text { Category }\end{array}$} & \multirow{2}{*}{$\begin{array}{c}\text { Plant } \\
\text { Damage } \\
\text { State }\end{array}$} & \multicolumn{7}{|c|}{ Accident Progression Characteristics } \\
\hline & & $\begin{array}{l}\text { Containment } \\
\text { Failure Time }\end{array}$ & $\begin{array}{l}\text { Containment } \\
\text { Failure Mode }\end{array}$ & $\mathbf{C C I}$ & Amt CCI & RCS Pres. & VB Mode & Sprays \\
\hline RZI & LOCA & CF at VB & Rupture & Prm Shlw & Medium & LowP & Pour & No \\
\hline RZ2 & LOCA & Late CF & Leak & Prm Dp & Large & LowP & Pour & No \\
\hline RZ3 & LOCA & No CF & No CF & Prm Dp & Large & LowP & Pour & Yes \\
\hline RZ4 & Bypass & No $C F$ & Bypass & Prm Dry & Large & LowP & Pour & No \\
\hline
\end{tabular}

Table A.4 Characteristics of Peach Bottom Release Categories

\begin{tabular}{|c|c|c|c|c|c|c|c|c|c|}
\hline \multirow{2}{*}{$\begin{array}{l}\text { Release } \\
\text { Category }\end{array}$} & \multirow{2}{*}{$\begin{array}{c}\text { Plant } \\
\text { Damage } \\
\text { State }\end{array}$} & \multicolumn{8}{|c|}{ Accident Progression Characteristics } \\
\hline & & $\begin{array}{l}\text { Containment } \\
\text { Failure Time }\end{array}$ & $\begin{array}{l}\text { Containment } \\
\text { Failure Mode }\end{array}$ & VB & DCH-SE & CCI & $\begin{array}{c}\text { DW } \\
\text { Sprays }\end{array}$ & $\begin{array}{c}\text { SP } \\
\text { Bypass }\end{array}$ & $\begin{array}{c}\text { RB } \\
\text { Bypass }\end{array}$ \\
\hline RPB I & LOSP & ECF (at VB) & DWMTH & HIP-nLPI & LoDCH & Dry CCI & No & NOBY & SMBY \\
\hline RPB2 & ATWS & ECF (at VB) & DWMTH & HIP-nLPI & LoDCH & Dry CCl & No & NOBY & SMBY \\
\hline RPB3 & ATWS & $\begin{array}{l}\text { ECF (CF during } \\
\text { or before core } \\
\text { damage) }\end{array}$ & WWVENT & LOP-nLPI & n-DCH-SE & Dry CCI & No & NOBY & LGBY \\
\hline RPB4 & LOSP & LCF & DWR & HIP-nLPI & LoDCH & Dry CCI & No & NOBY & LGBY \\
\hline RPB5 & LOSP & NCF & NCF & LOP-LPI & n-DCH-SE & FLD CCI & Yes & NOBY & SMBY \\
\hline RPB6 & LOSP & ECF (at VB) & DWMTH & HIP-nLPI & LoDCH & Dry $\mathrm{CCl}$ & No & NOBY & LGBY \\
\hline
\end{tabular}


Table A.5 Characteristics of Grand Gulf Release Categories

\begin{tabular}{|c|c|c|c|c|c|c|c|c|c|}
\hline \multirow{2}{*}{$\begin{array}{l}\text { Release } \\
\text { Category }\end{array}$} & \multirow{2}{*}{$\begin{array}{c}\text { Plant } \\
\text { Damage } \\
\text { State }\end{array}$} & \multicolumn{8}{|c|}{ Accident Progression Characteristics } \\
\hline & & $\begin{array}{l}\text { Containment } \\
\text { Failure Time }\end{array}$ & $\begin{array}{l}\text { Containment } \\
\text { Failure Mode }\end{array}$ & VB & DCH-SE & CCI & SP Bypass & Sprays & SRVBkr \\
\hline RGG1 & STSB & ECF (at VB) & Rupture & HIP-nLPI & LODCH & FLD CCI & Yes & No & Closed \\
\hline RGG2 & STSB & ECF (before VB) & Rupture & LOP-nLPI & LoE $\times S E$ & FLD CCI & No & No & Closed \\
\hline RGG3 & STSB & LCF & Rupture & LOP-nLPI & LoE $\times S E$ & FLD CCI & $\mathrm{L}$ & No & Closed \\
\hline RGG4 & STSB & $\mathrm{ECF}$ & Rupture & No VB & No DCH-SE & No $\mathrm{CCI}$ & Yes & No & Closed \\
\hline RGG5 & STSB & No $\mathrm{CF}$ & No CF & LOP-nLPI & LoE $\times S E$ & No $\mathrm{CCI}$ & No & No & Closed \\
\hline
\end{tabular}

Table A.6 Characteristics of LaSalle Release Categories

\begin{tabular}{|c|c|c|c|c|c|c|c|c|c|c|}
\hline \multirow{2}{*}{$\begin{array}{l}\text { Release } \\
\text { Category }\end{array}$} & \multirow{2}{*}{$\begin{array}{c}\text { Plant } \\
\text { Damage } \\
\text { State }\end{array}$} & \multicolumn{9}{|c|}{ Accident Progression Characteristics } \\
\hline & & $\begin{array}{l}\text { Containment } \\
\text { Failure Time }\end{array}$ & $\begin{array}{l}\text { Containment } \\
\text { Failure Mode }\end{array}$ & VB & DCH-SE & $\mathrm{CCI}$ & $\begin{array}{l}\text { DW } \\
\text { Sprays }\end{array}$ & $\begin{array}{l}\text { (Cavity } \\
\text { Failure } \\
\text { at VB) }\end{array}$ & $\begin{array}{c}\text { RB } \\
\text { Bypass }\end{array}$ & $\begin{array}{c}\text { SRV } \\
\text { Bypass }\end{array}$ \\
\hline RLASI & TRAN & ECF (before VB) & WWAWR & HIP-nLPI & n-DCH-SE & Dry CCl & No & Yes & No & nSRVBY \\
\hline RLAS2 & TRAN & ECF (at VB) & WWAW-LK & LOP-LPI & n-DCH-SE & INJ CCI & No & Yes & No & nSRVBY \\
\hline RLAS3 & TRAN & ECF (at VB) & DWR & LOP-LPI & LoE $\times S E$ & INJ CCI & No & Yes & No & nSRVBY \\
\hline RLAS4 & TRAN & ECF (before VB) & WWVENT & HIP-nLPI & n-DCH-SE & Dry $\mathrm{CCI}$ & No & Yes & No & n-SRVBY \\
\hline RLAS5 & TRAN & $\mathrm{LCF}$ & WWVENT & LOP-LPI & n-DCH-SE & INJ CCI & No & Yes & No & $n$-SRVBY \\
\hline RLAS6 & TRAN & LCF & CF-Ped & LOP-nLPI & n-DCH-SE & Dry CCI & No & Yes & Yes & n-SRVBY \\
\hline RLAS7 & TRAN & $\mathrm{NCF}$ & NCF & LOP-LPI & n-DCH-SE & INJ CCI & Yes & No & No & n-SRVBY \\
\hline
\end{tabular}


Table A.7 Radionuclide Release Characteristics into Environment for Surry

Building Height $-\mathbf{5 0} \mathrm{m}$

Building Width - $40 \mathrm{~m}$

\begin{tabular}{|c|c|c|c|c|c|c|c|c|c|c|c|c|c|c|c|}
\hline \multirow{2}{*}{$\begin{array}{l}\text { Release } \\
\text { Category }\end{array}$} & \multirow[t]{2}{*}{ Frequency } & \multirow{2}{*}{\begin{tabular}{|l|} 
Ele \\
(m)
\end{tabular}} & \multirow{2}{*}{$\begin{array}{c}\text { Energy } \\
\text { (W) }\end{array}$} & \multirow{2}{*}{\begin{tabular}{|c|} 
Time of \\
Core \\
Uncovery
\end{tabular}} & \multirow{2}{*}{$\begin{array}{c}\text { Time of } \\
\text { Release } \\
\text { (hrs) }\end{array}$} & \multirow{2}{*}{$\begin{array}{c}\text { Release } \\
\text { Duration }\end{array}$} & \multicolumn{9}{|c|}{ Fractional Releases } \\
\hline & & & & & & & NG & I & Cs & Te & $\mathbf{S r}$ & Ba & $\mathbf{R u}$ & La & Ce \\
\hline RSUR1 & $2.9 \mathrm{E}-7$ & \begin{tabular}{|l|}
10 \\
10 \\
\end{tabular} & $\begin{array}{l}2.8 \mathrm{E}+7 \\
2.8 \mathrm{E}+7 \\
\end{array}$ & $5.0 \mathrm{hrs}$ & $\begin{array}{c}6 \\
6.06\end{array}$ & $\begin{array}{c}200 \mathrm{sec} \\
2 \mathrm{hrs}\end{array}$ & $\begin{array}{l}1.0 \\
0.0\end{array}$ & $\begin{array}{l}0.25 \\
0.1\end{array}$ & $\begin{array}{l}0.18 \\
0.13\end{array}$ & $\begin{array}{l}0.08 \\
0.1 \\
\end{array}$ & $\begin{array}{l}0.02 \\
0.04 \\
\end{array}$ & $\begin{array}{l}0.02 \\
0.04 \\
\end{array}$ & $\begin{array}{l}0.005 \\
0.001\end{array}$ & $\begin{array}{l}0.001 \\
0.005\end{array}$ & $\begin{array}{l}0.005 \\
0.005\end{array}$ \\
\hline RSUR2 & $2.4 \mathrm{E}-6$ & $\mathbf{0}$ & 0 & $5.0 \mathrm{hrs}$ & 12 & $3 \mathrm{hrs}$ & 1.0 & 0.06 & 0.03 & 0.09 & 0.003 & 0.003 & 0.001 & $4.0 \mathrm{E}-4$ & 4.0E-4 \\
\hline RSUR3 & $3.3 \mathrm{E}-5$ & $\begin{array}{l}0 \\
0\end{array}$ & $\begin{array}{l}0 \\
0\end{array}$ & $5.0 \mathrm{hrs}$ & $\begin{array}{c}6 \\
16\end{array}$ & $\begin{array}{l}10 \mathrm{hrs} \\
10 \mathrm{hrs}\end{array}$ & $\begin{array}{l}2.5 \mathrm{E}-3 \\
2.5 \mathrm{E}-3\end{array}$ & $\begin{array}{l}1.5 \mathrm{E}-5 \\
1.5 \mathrm{E}-5 \\
\end{array}$ & $\begin{array}{l}1.2 \mathrm{E}-8 \\
1.2 \mathrm{E}-8 \\
\end{array}$ & $\begin{array}{l}7.5 \mathrm{E}-9 \\
7.5 \mathrm{E}-9 \\
\end{array}$ & $\begin{array}{l}2.5 \mathrm{E}-9 \\
2.5 \mathrm{E}-9\end{array}$ & $\begin{array}{l}2.5 \mathrm{E}-10 \\
2.5 \mathrm{E}-10 \\
\end{array}$ & $\begin{array}{l}2.0 \mathrm{E}-10 \\
2.0 \mathrm{E}-10\end{array}$ & \begin{tabular}{|}
$3.0 \mathrm{E}-10$ \\
$3.0 \mathrm{E}-10$
\end{tabular} & $\begin{array}{l}4.0 \mathrm{E}-10 \\
4.0 \mathrm{E}-10\end{array}$ \\
\hline RSUR4 & $1.6 \mathrm{E}-6$ & \begin{tabular}{|l|}
10 \\
10
\end{tabular} & $\begin{array}{l}2.8 E+7 \\
2.8 E+7\end{array}$ & $20 \mathrm{~min}$ & $\begin{array}{c}1 \\
1.5\end{array}$ & $\begin{array}{c}30 \mathrm{~min} \\
2 \mathrm{hrs}\end{array}$ & $\begin{array}{l}1.0 \\
0.0\end{array}$ & $\begin{array}{l}0.075 \\
0.04\end{array}$ & $\begin{array}{l}0.06 \\
0.06\end{array}$ & $\begin{array}{l}0.02 \\
0.05\end{array}$ & $\begin{array}{c}0.005 \\
0.02\end{array}$ & $\begin{array}{c}0.005 \\
0.02\end{array}$ & $\begin{array}{c}0.001 \\
6.0 \mathrm{E}-4\end{array}$ & $\begin{array}{l}.0 \mathrm{E}-4 \\
0.003\end{array}$ & $\begin{array}{l}0.001 \\
0.003\end{array}$ \\
\hline
\end{tabular}

Table A.8 Radionuclide Release Characteristics into Environment for Sequoyah

Building Height $-40 \mathrm{~m}$ Building Width $\cdot 40 \mathrm{~m}$

\begin{tabular}{|c|c|c|c|c|c|c|c|c|c|c|c|c|c|c|c|}
\hline \multirow{2}{*}{\begin{tabular}{|c|} 
Release \\
Category
\end{tabular}} & \multirow[t]{2}{*}{ Frequency } & \multirow{2}{*}{$\begin{array}{l}\text { Ele } \\
\text { (m) }\end{array}$} & \multirow{2}{*}{$\frac{\text { Energy }}{\text { (W) }}$} & \multirow{2}{*}{$\begin{array}{c}\text { Time of } \\
\text { Core } \\
\text { Uncovery }\end{array}$} & \multirow{2}{*}{$\begin{array}{c}\text { Time of } \\
\text { Release } \\
\text { (hrs) }\end{array}$} & \multirow{2}{*}{$\begin{array}{c}\text { Release } \\
\text { Duration }\end{array}$} & \multicolumn{9}{|c|}{ Fractional Releases } \\
\hline & & & & & & & NG & I & Cs & Te & $\mathbf{S r}$ & $\mathbf{B a}$ & $\mathbf{R u}$ & $\mathbf{L a}$ & Ce \\
\hline RSEQ1 & $2.8 \mathrm{E}-7$ & $\begin{array}{l}10 \\
10\end{array}$ & $\begin{array}{l}2.8 \mathrm{E}+7 \\
1.6 \mathrm{E}+6\end{array}$ & $5.0 \mathrm{hrs}$ & $\begin{array}{l}5.5 \\
6.0\end{array}$ & $\begin{array}{c}200 \mathrm{sec} \\
2 \mathrm{hrs}\end{array}$ & $\begin{array}{l}1.0 \\
0.0\end{array}$ & $\begin{array}{l}0.37 \\
0.22\end{array}$ & $\begin{array}{l}0.27 \\
0.35\end{array}$ & $\begin{array}{l}0.13 \\
0.30\end{array}$ & $\begin{array}{l}0.025 \\
0.13\end{array}$ & $\begin{array}{l}0.03 \\
0.12\end{array}$ & $\begin{array}{l}0.008 \\
0.003\end{array}$ & $\begin{array}{c}1.6 \mathrm{E}-3 \\
0.013\end{array}$ & $\begin{array}{l}0.008 \\
0.018\end{array}$ \\
\hline RSEQ2 & $3.6 E-6$ & $\begin{array}{l}10 \\
10\end{array}$ & $\begin{array}{l}2.8 \mathrm{E}+7 \\
1.6 \mathrm{E}+6\end{array}$ & $5.0 \mathrm{hrs}$ & $\begin{array}{l}6.0 \\
6.06\end{array}$ & $\begin{array}{c}200 \mathrm{sec} \\
2 \mathrm{hrs}\end{array}$ & $\begin{array}{l}1.0 \\
0.0\end{array}$ & $\begin{array}{l}0.05 \\
0.13 \\
\end{array}$ & $\begin{array}{l}0.04 \\
0.15 \\
\end{array}$ & $\begin{array}{l}0.02 \\
0.11 \\
\end{array}$ & $\begin{array}{l}0.004 \\
0.045 \\
\end{array}$ & $\begin{array}{l}0.004 \\
0.045\end{array}$ & $\begin{array}{l}0.001 \\
0.001\end{array}$ & $\begin{array}{c}2.0 \mathrm{E}-4 \\
0.005 \\
\end{array}$ & $\begin{array}{l}0.001 \\
0.006 \\
\end{array}$ \\
\hline RSEQ3 & $1.2 \mathrm{E}-5$ & 10 & $3.5 \mathrm{E}+6$ & $5.0 \mathrm{hrs}$ & 24.0 & $200 \mathrm{sec}$ & 1.0 & 0.033 & $6.5 \mathrm{E}-6$ & $3.2 \mathrm{E}-6$ & $1.5 \mathrm{E}-6$ & $1.5 \mathrm{E}-6$ & $3.2 \mathrm{E}-8$ & $1.6 \mathrm{E}-7$ & $1.6 \mathrm{E}-7$ \\
\hline RSEQ4 & $3.5 E-5$ & $\begin{array}{l}0 \\
0\end{array}$ & $\begin{array}{l}0 \\
0\end{array}$ & $5.0 \mathrm{hrs}$ & $\begin{array}{l}6.0 \\
16.0\end{array}$ & $\begin{array}{l}10 \mathrm{hrs} \\
10 \mathrm{hrs}\end{array}$ & $\begin{array}{l}2.5 \mathrm{E}-3 \\
2.5 \mathrm{E}-3\end{array}$ & $\begin{array}{l}1.5 \mathrm{E}-5 \\
1.5 \mathrm{E}-5\end{array}$ & $\begin{array}{l}1.0 \mathrm{E}-7 \\
1.0 \mathrm{E}-7\end{array}$ & $\begin{array}{l}7.0 \mathrm{E}-8 \\
7.0 \mathrm{E}-8\end{array}$ & $\begin{array}{l}2.0 \mathrm{E}-8 \\
2.0 \mathrm{E}-8 \\
\end{array}$ & $\begin{array}{l}2.0 \mathrm{E}-8 \\
2.0 \mathrm{E}-8\end{array}$ & $\begin{array}{l}1.9 \mathrm{E}-9 \\
1.8 \mathrm{E}-9 \\
\end{array}$ & $\begin{array}{l}2.5 \mathrm{E}-9 \\
2.5 \mathrm{E}-9 \\
\end{array}$ & $\begin{array}{l}4.0 \mathrm{E}-9 \\
4.0 \mathrm{E}-9 \\
\end{array}$ \\
\hline$\overline{\text { RSEQS }}$ & $3.1 \mathrm{E}-6$ & $\begin{array}{l}0 \\
0\end{array}$ & $\begin{array}{l}1.9 \mathrm{E}+6 \\
1.7 \mathrm{E}+5\end{array}$ & 20 mins & $\begin{array}{l}1.0 \\
1.5 \\
\end{array}$ & $\begin{array}{c}30 \mathrm{~min} \\
2 \mathrm{hrs}\end{array}$ & $\begin{array}{l}1.0 \\
0.0\end{array}$ & $\begin{array}{l}0.075 \\
0.04 \\
\end{array}$ & $\begin{array}{l}0.06 \\
0.06\end{array}$ & $\begin{array}{l}0.02 \\
0.05 \\
\end{array}$ & $\begin{array}{l}0.005 \\
0.02\end{array}$ & $\begin{array}{c}0.005 \\
0.02\end{array}$ & $\begin{array}{c}0.001 \\
6.0 \mathrm{E}-4\end{array}$ & $\begin{array}{c}3.0 \mathrm{E}-4 \\
0.003\end{array}$ & $\begin{array}{l}0.001 \\
0.003\end{array}$ \\
\hline
\end{tabular}


Table A.9 Radionuclide Release Characieristics into Environment for Zion

Building Height - $50 \mathrm{~m}$

Building Width - $40 \mathrm{~m}$

\begin{tabular}{|c|c|c|c|c|c|c|c|c|c|c|c|c|c|}
\hline \multirow{2}{*}{$\begin{array}{l}\text { Relense } \\
\text { Category }\end{array}$} & \multirow{2}{*}{ Frequency } & \multirow{2}{*}{$\begin{array}{c}\text { Time of } \\
\text { Core } \\
\text { Uncovery }\end{array}$} & \multirow{2}{*}{$\begin{array}{l}\text { Time of } \\
\text { Relense }\end{array}$} & \multirow{2}{*}{$\begin{array}{l}\text { Release } \\
\text { Duration }\end{array}$} & \multicolumn{9}{|c|}{ Fractional Relenses } \\
\hline & & & & & NG & 1 & Cs & Te & $\mathbf{S r}$ & Ba & $\mathbf{R u}$ & $\mathbf{L a}$ & Ce \\
\hline $\mathbf{R Z 1}$ & $4.6 \times 10^{-6}$ & $5.0 \mathrm{hrs}$ & $6.0 \mathrm{hrs}$ & $\begin{array}{c}10 \mathrm{sec} \\
2 \mathrm{hrs} \\
\end{array}$ & $\begin{array}{l}1.0 \\
0.0 \\
\end{array}$ & $\begin{array}{l}0.25 \\
0.02 \\
\end{array}$ & $\begin{array}{l}0.18 \\
0.03 \\
\end{array}$ & $\begin{array}{l}0.08 \\
0.02 \\
\end{array}$ & $\begin{array}{l}0.02 \\
0.01 \\
\end{array}$ & $\begin{array}{l}0.02 \\
0.01\end{array}$ & $\begin{array}{c}0.005 \\
0.0002 \\
\end{array}$ & $\begin{array}{l}0.001 \\
0.001 \\
\end{array}$ & $\begin{array}{l}0.005 \\
0.001 \\
\end{array}$ \\
\hline RZ2 & $7.8 \times 10^{-5}$ & $5.0 \mathrm{hrs}$ & $12 \mathrm{hrs}$ & $3 \mathrm{hrs}$ & 1.0 & 0.03 & $6 \times 10^{-6}$ & $7 \times 10^{-6}$ & $10^{-6}$ & $10^{-6}$ & $2 \times 10^{-8}$ & $10^{-7}$ & $10^{-7}$ \\
\hline RZ3 & $2.4 \times 10^{-4}$ & $5.0 \mathrm{hrs}$ & $6.0 \mathrm{hrs}$ & $24 \mathrm{hrs}$ & 0.005 & $3 \times 10^{0}$ & $2.4 \times 10^{-2}$ & $1.5 \times 10^{-6}$ & $5 \times 10$ & $5 \times 10$ & $4 \times 10$ & $6 \times 10^{-}$ & $8 \times 10^{0}$ \\
\hline $\mathbf{R Z 4}$ & $2.3 \times 10^{-6}$ & 20 mins & $1.0 \mathrm{hr}$ & $\begin{array}{c}30 \mathrm{~min} \\
2 \mathrm{hrs}\end{array}$ & $\begin{array}{l}1.0 \\
0.0\end{array}$ & $\begin{array}{c}0.075 \\
0.04\end{array}$ & $\begin{array}{l}0.06 \\
0.06\end{array}$ & $\begin{array}{l}0.02 \\
0.05\end{array}$ & $\begin{array}{c}0.005 \\
0.02\end{array}$ & $\begin{array}{c}0.005 \\
0.02\end{array}$ & $\begin{array}{c}0.001 \\
6 \times 10^{-4}\end{array}$ & $\begin{array}{c}3 \times 10^{-4} \\
0.003\end{array}$ & $\begin{array}{l}0.001 \\
0.003 \\
\end{array}$ \\
\hline
\end{tabular}

Table A.10 Radionuclide Release Characteristics into Environment for Peach Bottom

Building Height - $50 \mathrm{~m}$

Building Width - $50 \mathrm{~m}$

\begin{tabular}{|c|c|c|c|c|c|c|c|c|c|c|c|c|c|c|c|}
\hline \multirow{2}{*}{$\begin{array}{c}\text { Release } \\
\text { Category }\end{array}$} & \multirow[t]{2}{*}{ Frequency } & \multirow{2}{*}{$\begin{array}{l}\text { Ele } \\
\text { (m) }\end{array}$} & \multirow{2}{*}{$\begin{array}{c}\text { Energy } \\
\text { (W) }\end{array}$} & \multirow{2}{*}{$\begin{array}{c}\text { Time of } \\
\text { Accident } \\
\text { Recognition }\end{array}$} & \multirow{2}{*}{$\begin{array}{c}\text { Time of } \\
\text { Release } \\
\text { (hrs) }\end{array}$} & \multirow{2}{*}{$\begin{array}{c}\text { Release } \\
\text { Duration }\end{array}$} & \multicolumn{9}{|c|}{ Fractional Releases } \\
\hline & & & & & & & NG & 1 & Cs & Te & Sr & $\mathbf{B a}$ & Ru & La & $\mathrm{Ce}$ \\
\hline RPB I & $1.2 \mathrm{E}-6$ & $\begin{array}{l}30 \\
30\end{array}$ & $\begin{array}{l}6.4 \mathrm{E}+7 \\
3.7 \mathrm{E}+5 \\
\end{array}$ & $8.0 \mathrm{hrs}$ & $\begin{array}{r}11.5 \\
11.75 \\
\end{array}$ & $\begin{array}{l}15 \mathrm{~min} \\
4 \mathrm{hrs}\end{array}$ & $\begin{array}{l}0.76 \\
0.24 \\
\end{array}$ & $\begin{array}{l}0.01 \\
0.1\end{array}$ & $\begin{array}{l}0.01 \\
0.09\end{array}$ & $\begin{array}{c}0.003 \\
0.07\end{array}$ & \begin{tabular}{c|}
0.001 \\
0.04
\end{tabular} & $\begin{array}{l}1.2 \mathrm{E}-3 \\
0.03\end{array}$ & $\begin{array}{l}1.2 \mathrm{E}-3 \\
6.0 \mathrm{E}-4\end{array}$ & $\begin{array}{l}3.0 \mathrm{E}-4 \\
0.003\end{array}$ & $\begin{array}{c}3.0 \mathrm{E}-4 \\
0.004\end{array}$ \\
\hline RPB2 & E-6 & $\begin{array}{l}30 \\
30\end{array}$ & $\begin{array}{l}6.4 E+7 \\
2.3 E+5\end{array}$ & $4.8 \mathrm{hrs}$ & $\begin{array}{c}7.3 \\
7.55 \\
\end{array}$ & $\begin{array}{l}15 \mathrm{~min} \\
4 \mathrm{hrs}\end{array}$ & $\begin{array}{l}0.76 \\
0.24 \\
\end{array}$ & $\begin{array}{l}0.01 \\
0.1\end{array}$ & $\begin{array}{l}0.01 \\
0.09\end{array}$ & $\begin{array}{c}0.003 \\
0.07\end{array}$ & $\begin{array}{c}0.001 \\
0.04 \\
\end{array}$ & $\begin{array}{l}1.2 \mathrm{E}-3 \\
0.03 \\
\end{array}$ & $\begin{array}{l}1.2 \mathrm{E}-3 \\
6.0 \mathrm{E}-4\end{array}$ & $\begin{array}{c}3.0 \mathrm{E}-4 \\
0.003\end{array}$ & $\begin{array}{c}3.0 \mathrm{E}-4 \\
0.004 \\
\end{array}$ \\
\hline RPB3 & $6 E-6$ & $\begin{array}{l}30 \\
30\end{array}$ & $\begin{array}{l}0 \\
0 \\
\end{array}$ & $4.8 \mathrm{hrs}$ & $\begin{array}{l}4.8 \\
7.3 \\
\end{array}$ & $\begin{array}{c}2.5 \mathrm{hrs} \\
4 \mathrm{hrs} \\
\end{array}$ & $\begin{array}{l}0.76 \\
0.24 \\
\end{array}$ & \begin{tabular}{|c|}
$2.3 \mathrm{E}-3$ \\
0.04 \\
\end{tabular} & $\begin{array}{c}0.001 \\
0.04 \\
\end{array}$ & $\begin{array}{c}1.5 E-3 \\
0.03 \\
\end{array}$ & $\begin{array}{c}5.0 \mathrm{E}-4 \\
0.02\end{array}$ & $\begin{array}{c}6.0 \mathrm{E}-4 \\
0.02\end{array}$ & $\begin{array}{l}5.0 \mathrm{E}-5 \\
3.0 \mathrm{E}-4\end{array}$ & $\begin{array}{l}1.0 \mathrm{E}-5 \\
1.5 \mathrm{E}-3 \\
\end{array}$ & $\begin{array}{c}5.0 \mathrm{E}-5 \\
0.002 \\
\end{array}$ \\
\hline RPB4 & 2E-7 & 30 & $7.7 \mathrm{E}+6$ & $8.0 \mathrm{hrs}$ & 13.0 & $15 \mathrm{~min}$ & 1.0 & 0.01 & 5.4E-3 & 0.004 & $2.2 \mathrm{E}-3$ & $1.7 E-3$ & $1.0 \mathrm{E}-4$ & $1.8 \mathrm{E}-4$ & $2.3 \mathrm{E}-4$ \\
\hline RPB5 & $8 E-7$ & $\begin{array}{l}30 \\
30\end{array}$ & $\begin{array}{l}1.5 E+5 \\
2.5 E+5\end{array}$ & $8.0 \mathrm{hrs}$ & $\begin{array}{l}13.0 \\
15.5 \\
\end{array}$ & $\begin{array}{r}2.5 \\
6 \mathrm{hrs} \\
\end{array}$ & $\begin{array}{l}2.5 \mathrm{E}-3 \\
2.5 \mathrm{E}-3 \\
\end{array}$ & \begin{tabular}{|l|}
$5.6 E-4$ \\
$5.6 E-4$
\end{tabular} & $\begin{array}{l}6.0 \mathrm{E}-9 \\
6.0 \mathrm{E}-9\end{array}$ & $\begin{array}{l}2.6 \mathrm{E}-\bar{y} \\
2.6 \mathrm{E}-9 \\
\end{array}$ & $\begin{array}{l}2.8 \mathrm{E}-9 \\
2.8 \mathrm{E}-9\end{array}$ & $\begin{array}{l}2.2 \mathrm{E}-9 \\
2.2 \mathrm{E}-9 \\
\end{array}$ & $\begin{array}{l}1.5 \mathrm{E}-11 \\
1.5 \mathrm{E}-11\end{array}$ & $\begin{array}{l}1.5 \mathrm{E}-11 \\
1.5 \mathrm{E}-11 \\
\end{array}$ & $\begin{array}{l}2.9 \mathrm{E}-10 \\
2.9 \mathrm{E}-10 \\
\end{array}$ \\
\hline RPB6 & $3 E-8$ & $\begin{array}{l}30 \\
30\end{array}$ & $\begin{array}{l}7.7 \mathrm{E}+6 \\
1.9 \mathrm{E}+6\end{array}$ & $8.0 \mathrm{hrs}$ & $\begin{array}{c}11.5 \\
11.75\end{array}$ & $\begin{array}{l}15 \mathrm{~min} \\
4 \mathrm{hrs}\end{array}$ & $\begin{array}{l}0.76 \\
0.24\end{array}$ & $\begin{array}{l}0.04 \\
0.4\end{array}$ & $\begin{array}{l}0.04 \\
0.36\end{array}$ & $\begin{array}{c}0.012 \\
0.28\end{array}$ & \begin{tabular}{c|}
0.004 \\
0.16
\end{tabular} & $\begin{array}{c}0.005 \\
0.12\end{array}$ & $\begin{array}{c}0.005 \\
2.4 E-3\end{array}$ & $\begin{array}{c}1.2 \mathrm{E}-3 \\
0.01\end{array}$ & $\begin{array}{c}1.2 \mathrm{E}-3 \\
0.02\end{array}$ \\
\hline
\end{tabular}


Table A.11 Radionuclide Release Characteristics into Environment for Grand Gulf

Building Height $-60 \mathrm{~m}$

Building Width $-\mathbf{4 0} \mathrm{m}$

\begin{tabular}{|c|c|c|c|c|c|c|c|c|c|c|c|c|c|}
\hline \multirow{2}{*}{$\begin{array}{l}\text { Release } \\
\text { Category }\end{array}$} & \multirow[t]{2}{*}{ Frequency } & \multirow{2}{*}{$\begin{array}{c}\text { Time of } \\
\text { Accident } \\
\text { Recognition" }\end{array}$} & \multirow{2}{*}{$\begin{array}{c}\text { Time of } \\
\text { Release } \\
\text { (hrs) }\end{array}$} & \multirow{2}{*}{$\begin{array}{c}\text { Release } \\
\text { Duration }\end{array}$} & \multicolumn{9}{|c|}{ Fractional Releases } \\
\hline & & & & & NG & I & Cs & Te & $\mathbf{S r}$ & $\mathbf{B a}$ & $\mathbf{R u}$ & La & Ce \\
\hline RGG1 & $8.4 \times 10^{-7}$ & $1.0 \mathrm{hr}$ & 3.6 & $\begin{array}{l}3 \mathrm{~min} \\
4 \mathrm{hrs}\end{array}$ & $\begin{array}{l}0.75 \\
0.25\end{array}$ & $\begin{array}{l}0.03 \\
0.2 \\
\end{array}$ & $\begin{array}{l}0.03 \\
0.08\end{array}$ & $\begin{array}{l}8 \times 10^{-3} \\
0.04\end{array}$ & $\begin{array}{c}4 \times 10^{-3} \\
0.02\end{array}$ & \begin{tabular}{|c|}
$5 \times 10^{-3}$ \\
0.02 \\
\end{tabular} & $\begin{array}{l}5 \times 10^{-3} \\
2 \times 10^{-4}\end{array}$ & $\begin{array}{c}1.4 \times 10^{-3} \\
10^{-3} \\
\end{array}$ & $\begin{array}{c}1.4 \times 10^{-3} \\
2 \times 10^{-3} \\
\end{array}$ \\
\hline RGG2 & $9.2 \times 10^{-7}$ & $1.0 \mathrm{hr}$ & 2.3 & $\begin{array}{c}1.3 \mathrm{hrs} \\
4 \mathrm{hrs}\end{array}$ & $\begin{array}{l}0.75 \\
0.25\end{array}$ & $\begin{array}{c}5 \times 10^{-3} \\
0.03\end{array}$ & $\begin{array}{c}5 \times 10^{-3} \\
0.01 \\
\end{array}$ & $\begin{array}{l}2 \times 10^{-3} \\
8 \times 10^{-3} \\
\end{array}$ & $\begin{array}{l}7 \times 10^{-4} \\
4 \times 10^{-3} \\
\end{array}$ & $\begin{array}{l}6 \times 10^{-4} \\
3 \times 10^{-3} \\
\end{array}$ & $\begin{array}{l}4 \times 10^{-4} \\
3 \times 10^{-5} \\
\end{array}$ & \begin{tabular}{|l|}
$1.2 \times 10^{-4}$ \\
$1.6 \times 10^{-4}$ \\
\end{tabular} & \begin{tabular}{|c|c|}
$.8 \times 10^{-4}$ \\
$3 \times 10^{-4}$ \\
\end{tabular} \\
\hline RGG3 & $1.16 \times 10^{-6}$ & $1.0 \mathrm{hr}$ & 14.0 & $\begin{array}{l}3 \mathrm{~min} \\
4 \mathrm{hrs}\end{array}$ & $\begin{array}{l}0.9 \\
0.1\end{array}$ & $\begin{array}{l}0.13 \\
0.015\end{array}$ & $\begin{array}{c}0.01 \\
0.001\end{array}$ & $\begin{array}{l}7 \times 10^{-3} \\
8 \times 10^{-4}\end{array}$ & $\begin{array}{l}3.5 \times 10^{-} \\
4 \times 10^{-4} \\
\end{array}$ & $\begin{array}{l}3 \times 10^{-3} \\
3 \times 10^{-4}\end{array}$ & $\begin{array}{l}3 \times 10^{-4} \\
3 \times 10^{-5}\end{array}$ & $\begin{array}{l}2 \times 10^{-4} \\
2 \times 10^{-5}\end{array}$ & $\begin{array}{l}3 \times 10^{-4} \\
3 \times 10^{-5}\end{array}$ \\
\hline RGG4 & $7.3 \times 10^{-7}$ & $1.0 \mathrm{hr}$ & 2.3 & $\begin{array}{l}1.3 \mathrm{hrs} \\
4 \mathrm{hrs}\end{array}$ & $\begin{array}{l}1.0 \\
0.0\end{array}$ & $\begin{array}{c}3 \times 10^{-3} \\
0.03\end{array}$ & $\begin{array}{c}2.5 \times 10^{-3} \\
0.015\end{array}$ & $\begin{array}{c}1.3 \times 10^{-3} \\
6 \times 10^{-3}\end{array}$ & $\begin{array}{c}3 \times 10^{-4} \\
0.0 \\
\end{array}$ & $\begin{array}{c}3.6 \times 10^{-4} \\
0.0\end{array}$ & $\begin{array}{c}9 \times 10^{-5} \\
0.0\end{array}$ & \begin{tabular}{|c|}
$1.5 \times 10^{-5}$ \\
0.0
\end{tabular} & $\begin{array}{c}8 \times 10^{-5} \\
0.0\end{array}$ \\
\hline RGG5 & $2.4 \times 10^{-7}$ & $1.0 \mathrm{hr}$ & 14.0 & $\begin{array}{l}2 \mathrm{hrs} \\
6 \mathrm{hrs}\end{array}$ & $\begin{array}{l}2.5 \times 10^{-3} \\
2.5 \times 10^{-3}\end{array}$ & $\begin{array}{l}3.7 \times 10^{-6} \\
3.7 \times 10^{-6}\end{array}$ & $\begin{array}{l}3.5 \times 10^{-9} \\
3.5 \times 10^{-9}\end{array}$ & $\begin{array}{l}1.4 \times 10^{-9} \\
1.4 \times 10^{-9}\end{array}$ & $\begin{array}{l}5 \times 10^{-10} \\
5 \times 10^{-10}\end{array}$ & $\begin{array}{l}4 \times 10^{-10} \\
4 \times 10^{-10}\end{array}$ & $\begin{array}{l}2.8 \times 10^{-10} \\
2.8 \times 10^{-10}\end{array}$ & $8.5 \times 10^{-11}$ & $\begin{array}{l}1.3 \times 10^{-10} \\
1.3 \times 10^{-10}\end{array}$ \\
\hline
\end{tabular}

-Time when the reactor water level reaches two feet above bottom of the active fuel. 
-Time when the reactor water level reaches two feet above bottom of the active fuel. 


\section{APPENDIX B - AUXILIARY DOSE INFORMATION}


Appendix B Auxiliary Dose Information

Table B.2.1

Centerline Dose (Rem) to Red Marrow as a function of distance by pathway for the MPB! source term. The third column is the dose to the average (weighted) population, the fourth column is the dose to the evacuees following Mean evacuation, and the last column is the dose to the non-evacuees.

\begin{tabular}{|c|c|c|c|c|}
\hline Organ/Pathway & Distance & $\begin{array}{c}\text { Dose to } \\
\text { Average } \\
\text { Population }\end{array}$ & $\begin{array}{c}\text { Dose to } \\
\text { Evacuee } \\
\text { Population }\end{array}$ & $\begin{array}{c}\text { Dose to } \\
\text { Non-Evacuee } \\
\text { Population }\end{array}$ \\
\hline Red Marrow Cloudshine & $0 .-0.25 \mathrm{mi}$ & 0.88 & 0.023 & 172 \\
\hline Red Marrow Cloudshine & $0.25-0.33 \mathrm{mi}$ & 0.64 & 0.032 & 121 \\
\hline Red Marrow Cloudshine & $0.33-0.4 \mathrm{mi}$ & 0.57 & 0.035 & 108 \\
\hline Red Marrow Cloudshine & $0.4-0.5 \mathrm{mi}$ & 0.52 & 0.038 & 96.4 \\
\hline Red Marrow Cloudshine & $0.5-1.25 \mathrm{mi}$ & 0.36 & 0.042 & 63.4 \\
\hline Red Marrow Cloudshine & $1.25-1.33 \mathrm{mi}$ & 0.28 & 0.044 & 46.3 \\
\hline Red Marrow Cloudshine & $1.33-1.4 \mathrm{mi}$ & 0.27 & 0.044 & 44.2 \\
\hline Red Marrow Cloudshine & $1.4-1.5 \mathrm{mi}$ & 0.25 & 0.044 & 42.1 \\
\hline Red Marrow Cloudshine & $1.5-2 \mathrm{mi}$ & 0.22 & 0.044 & 34.4 \\
\hline Red Marrow Groundshine & $0-0.25 \mathrm{mi}$ & 12.60 & 0.008 & 2510 \\
\hline Red Marrow Groundshine & $0.25-0.33 \mathrm{mi}$ & 6.55 & 0.007 & 1310 \\
\hline Red Marrow Groundshine & $0.33-0.4 \mathrm{mi}$ & 5.37 & 0.007 & 1070 \\
\hline Red Marrow Groundshine & $0.4-0.5 \mathrm{mi}$ & 4.44 & 0.007 & 887 \\
\hline Red Marrow Groundshine & $0.5-1.25 \mathrm{mi}$ & 2.26 & 0.005 & 451 \\
\hline Red Marrow Groundshine & $1.25-1.33 \mathrm{mi}$ & 1.40 & 0.004 & 280 \\
\hline Red Marrow Groundshine & $1.33-1.4 \mathrm{mi}$ & 1.31 & 0.004 & 261 \\
\hline Red Marrow Groundshine & $1.4-1.5 \mathrm{mi}$ & 1.21 & 0.004 & 241 \\
\hline Red Marrow Groundshine & $1.5-2 \mathrm{mi}$ & 0.90 & 0.003 & 179 \\
\hline Red Marrow Inhalation Acute & $0-0.25 \mathrm{mi}$ & 4.04 & 0.082 & 792 \\
\hline Red Marrow Inhalation Acute & $0.25-0.33 \mathrm{mi}$ & 2.14 & 0.076 & 413 \\
\hline Red Marrow Inhalation Acute & $0.33-0.4 \mathrm{mi}$ & 1.77 & 0.073 & 339 \\
\hline Red Marrow Inhalation Acute & $0.4-0.5 \mathrm{mi}$ & 1.47 & 0.070 & 280 \\
\hline Red Marrow Inhalation Acute & $0.5-1.25 \mathrm{mi}$ & 0.77 & 0.057 & 143 \\
\hline Red Marrow Inhalation Acute & $1.25-1.33 \mathrm{mi}$ & 0.49 & 0.048 & 88.5 \\
\hline
\end{tabular}


Table B.2.1 (continued)

Appendix B Auxiliary Dose Information

\begin{tabular}{|c|c|c|c|c|}
\hline Organ/Pathway & Distance & $\begin{array}{c}\text { Dose to } \\
\text { Average } \\
\text { Population }\end{array}$ & $\begin{array}{c}\text { Dose to } \\
\text { Evacuee } \\
\text { Population }\end{array}$ & $\begin{array}{c}\text { Dose to } \\
\text { Non-Evacuee } \\
\text { Population }\end{array}$ \\
\hline Red Marrow Inhalation Acute & $1.33-1.4 \mathrm{mi}$ & 0.46 & 0.046 & 82.4 \\
\hline Red Marrow Inhalation Acute & $1.4-1.5 \mathrm{mi}$ & 0.43 & 0.045 & 76.2 \\
\hline Red Marrow Inhalation Acute & $1.5-2 \mathrm{mi}$ & 0.32 & 0.041 & 56.2 \\
\hline Red Marrow Resuspension Acute & $0-0.25 \mathrm{mi}$ & 0.11 & 0 & 22.1 \\
\hline Red Marrow Resuspension Acute & $0.25-0.33 \mathrm{mi}$ & 0.06 & 0 & 11.5 \\
\hline Red Marrow Resuspension Acute & $0.33-0.4 \mathrm{mi}$ & 0.05 & 0 & 9.44 \\
\hline Red Marrow Resuspension Acute & $0.40 .5 \mathrm{mi}$ & 0.04 & 0 & 7.81 \\
\hline Red Marrow Resuspension Acute & $0.5-1.25 \mathrm{mi}$ & 0.02 & 0 & 3.97 \\
\hline Red Marrow Resuspension Acute & $1.25-1.33 \mathrm{mi}$ & 0.01 & 0 & 2.47 \\
\hline Red Marrow Resuspension Acute & $1.33-1.4 \mathrm{mi}$ & 0.01 & 0 & 2.3 \\
\hline Red Marrow Resuspension Acute & $1.41 .5 \mathrm{mi}$ & 0.01 & $0^{\circ}$ & 2.13 \\
\hline Red Marrow Resuspension Acute & $1.5-2 \mathrm{mi}$ & 0.01 & 0 & 1.58 \\
\hline Red Marrow Total & $0.0 .25 \mathrm{mi}$ & 17.60 & 0.113 & 3500 \\
\hline Red Marrow Total & $0.25-0.33 \mathrm{mi}$ & 9.38 & 0.116 & 1850 \\
\hline Red Marrow Total & $0.33-0.4 \mathrm{mi}$ & 7.75 & 0.116 & 1530 \\
\hline Red Marrow Total & $0.40 .5 \mathrm{mi}$ & 6.47 & 0.114 & 1270 \\
\hline Red Marrow Total & $0.5-1.25 \mathrm{mi}$ & 3.41 & 0.105 & 661 \\
\hline Red Marrow Total & $1.25-1.33 \mathrm{mi}$ & 2.18 & 0.096 & 417 \\
\hline Red Marrow Total & $1.33-1.4 \mathrm{mi}$ & 2.04 & 0.094 & 390 \\
\hline Red Marrow Total & $1.4-1.5 \mathrm{mi}$ & 1.90 & 0.093 & 362 \\
\hline Red Marrow Total & $1.5-2 \mathrm{mi}$ & 1.44 & 0.088 & 271 \\
\hline
\end{tabular}


Appendix B Auxiliary Dose Information

Table B.2.2

Centerline Dose (Rem) to Lungs as a function of distance by pathway for the MPBI source term. The third column is the dose to the average (weighted) population, the fourth column is the dose to the evacuees following Mean evacuation, and the last column is the dose to the non-evacuees.

\begin{tabular}{|c|c|c|c|c|}
\hline Organ/Pathway & Distance & $\begin{array}{c}\text { Dose to } \\
\text { Average } \\
\text { Population }\end{array}$ & $\begin{array}{c}\text { Dose to } \\
\text { Evacuee } \\
\text { Population }\end{array}$ & $\begin{array}{c}\text { Dose to } \\
\text { Non-Evacuee } \\
\text { Population }\end{array}$ \\
\hline Lungs Cloudshine & $0-0.25 \mathrm{mi}$ & 0.88 & 0.024 & 170 \\
\hline Lungs Cloudshine & $0.25-0.33 \mathrm{mi}$ & 0.63 & 0.034 & 120 \\
\hline Lumgs Cloudshine & $0.33-0.4 \mathrm{mi}$ & 0.57 & 0.037 & 107 \\
\hline Lungs Cloudshine & $0.4-0.5 \mathrm{mi}$ & 0.52 & 0.039 & 95.6 \\
\hline Lungs Cloudshine & $0.5-1.25 \mathrm{mi}$ & 0.36 & 0.044 & 62.9 \\
\hline Lungs Cloudshine & $1.25-1.33 \mathrm{mi}$ & 0.28 & 0.046 & 46 \\
\hline Lungs Cloudshine & $1.33-1.4 \mathrm{mi}$ & 0.27 & 0.046 & 43.9 \\
\hline Lungs Cloudshine & $1.4-1.5 \mathrm{mi}$ & 0.26 & 0.046 & 41.8 \\
\hline Lungs Cloudshine & $1.5-2 \mathrm{mi}$ & 0.22 & 0.046 & 34.2 \\
\hline Lungs Groundshine & $0-0.25 \mathrm{mi}$ & 12.4 & 0.008 & 2480 \\
\hline Lungs Groundshine & $0.25-0.33 \mathrm{mi}$ & 6.46 & 0.007 & 1290 \\
\hline Lungs Groundshine & $0.33-0.4 \mathrm{mi}$ & 5.30 & 0.007 & 1060 \\
\hline Lungs Groundshine & $0.4-0.5 \mathrm{mi}$ & 4.39 & 0.007 & 876 \\
\hline Lungs Groundshine & $0.5-1.25 \mathrm{mi}$ & 2.23 & 0.005 & 445 \\
\hline Lungs Groundshine & $1.25-1.33 \mathrm{mi}$ & 1.39 & 0.004 & 276 \\
\hline Lungs Groundshine & $1.33-1.4 \mathrm{mi}$ & 1.29 & 0.004 & 257 \\
\hline Lungs Groundshine & $1.4-1.5 \mathrm{mi}$ & 1.20 & 0.004 & 238 \\
\hline Lungs Groundshine & $1.5-2 \mathrm{mi}$ & 0.89 & 0.003 & 177 \\
\hline Lumgs Inhalation Acute & $0-0.25 \mathrm{mi}$ & 20.80 & 0.580 & 4050 \\
\hline Lungs Inhalation Acute & $0.25-0.33 \mathrm{mi}$ & 11.10 & 0.536 & 2110 \\
\hline Lungs Inhalation Acute & $0.33-0.4 \mathrm{mi}$ & 9.16 & 0.517 & 1730 \\
\hline Lungs Inhalation Acute & $0.4-0.5 \mathrm{mi}$ & 7.65 & 0.496 & 1430 \\
\hline Lungs Inhalation Acute & $0.5-1.25 \mathrm{mi}$ & 4.05 & 0.408 & 728 \\
\hline Lungs Inhalation Acute & $1.25-1.33 \mathrm{mi}$ & 2.59 & 0.343 & 451 \\
\hline
\end{tabular}


Table B.2.2 (continued)

Appendix B Auxiliary Dose Information

\begin{tabular}{||l|l|c|c|c||}
\hline \multicolumn{1}{|c|}{ Organ/Pathway } & \multicolumn{1}{|c|}{ Distance } & $\begin{array}{c}\text { Dose to } \\
\text { Average } \\
\text { Population }\end{array}$ & $\begin{array}{c}\text { Dose to } \\
\text { Evacuee } \\
\text { Population }\end{array}$ & $\begin{array}{c}\text { Dose to } \\
\text { Non-Evacuee } \\
\text { Population }\end{array}$ \\
\hline Lungs Inhalation Acute & $1.33-1.4 \mathrm{mi}$ & 2.43 & 0.337 & 419 \\
\hline Lungs Inhalation Acute & $1.4-1.5 \mathrm{mi}$ & 2.27 & 0.330 & 388 \\
\hline Lungs Inhalation Acute & $1.5-2 \mathrm{mi}$ & 1.73 & 0.303 & 286 \\
\hline Lungs Resuspension Acute & $0-0.25 \mathrm{mi}$ & 0.57 & 0 & 114 \\
\hline Lungs Resuspension Acute & $0.25-0.33 \mathrm{mi}$ & 0.30 & 0 & 59.2 \\
\hline Lungs Resuspension Acute & $0.33-0.4 \mathrm{mi}$ & 0.24 & 0 & 48.5 \\
\hline Lungs Resuspension Acute & $0.4-0.5 \mathrm{mi}$ & 0.20 & 0 & 40.1 \\
\hline Lungs Resuspension Acute & $0.5-1.25 \mathrm{mi}$ & 0.10 & 0 & 20.4 \\
\hline Lungs Resuspension Acute & $1.25-1.33 \mathrm{mi}$ & 0.06 & 0 & 12.7 \\
\hline Lungs Resuspension Acute & $1.33-1.4 \mathrm{mi}$ & 0.06 & 0 & 11.8 \\
\hline Lungs Resuspension Acute & $1.4-1.5 \mathrm{mi}$ & 0.05 & 0 & 10.9 \\
\hline Lungs Resuspension Acute & $1.5-2 \mathrm{mi}$ & 0.04 & 0 & 8.1 \\
\hline Lungs Total Acute & $0-0.25 \mathrm{mi}$ & 34.70 & 0.612 & 6820 \\
\hline Lungs Total Acute & $0.25-0.33 \mathrm{mi}$ & 18.50 & 0.578 & 3580 \\
\hline Lungs Total Acute & $0.33-0.4 \mathrm{mi}$ & 15.30 & 0.561 & 2940 \\
\hline Lungs Total Acute & $0.4-0.5 \mathrm{mi}$ & 12.80 & 0.542 & 2440 \\
\hline Lungs Total Acute & $0.5-1.25 \mathrm{mi}$ & 6.74 & 0.457 & 1260 \\
\hline Lungs Total Acute & $1.25-1.33 \mathrm{mi}$ & 4.32 & 0.393 & 786 \\
\hline Lungs Total Acute & $1.33-1.4 \mathrm{mi}$ & 4.05 & 0.387 & 733 \\
\hline Lungs Total Acute & $1.4-1.5 \mathrm{mi}$ & 3.77 & 0.380 & 679 \\
\hline Lungs Total Acute & $1.5-2 \mathrm{mi}$ & 2.88 & 0.352 & 505 \\
\hline
\end{tabular}


Appendix B Auxiliary Dose Information

Table B.2.3

Centerline Dose (Rem) to Lower Large Intestine as a function of distance by pathway for the MPBI source term. The third column is the dose to the average (weighted) population, the fourth column is the dose to the evacuees following Mean EP evacuation, and the last column is the dose to the non-evacuees.

\begin{tabular}{|c|c|c|c|c|}
\hline Organ/Pathway & Distance & $\begin{array}{c}\text { Dose to } \\
\text { Average } \\
\text { Population }\end{array}$ & $\begin{array}{c}\text { Dose to } \\
\text { Evacuee } \\
\text { Population }\end{array}$ & $\begin{array}{c}\text { Dose to Non- } \\
\text { Evacuee } \\
\text { Population }\end{array}$ \\
\hline Lower LI Cloudshine & $0-0.25 \mathrm{mi}$ & 0.79 & 0.021 & 154 \\
\hline Lower LI Cloudshine & $0.25-0.33 \mathrm{mi}$ & 0.57 & 0.03 & 109 \\
\hline Lower LI Cloudshine & $0.33-0.4 \mathrm{mi}$ & 0.52 & 0.033 & 97 \\
\hline Lower LI Cloudshine & $0.40 .5 \mathrm{mi}$ & 0.47 & 0.035 & 87 \\
\hline Lower LI Cloudshine & $0.5-1.25 \mathrm{mi}$ & 0.32 & 0.039 & 57 \\
\hline Lower LI Cloudshine & $1.25-1.33 \mathrm{mi}$ & 0.25 & 0.041 & 42 \\
\hline Lower LI Cloudshine & $1.22-1.4 \mathrm{mi}$ & 0.24 & 0.041 & 40 \\
\hline Lower LI Cloudshine & $1.4-1.5 \mathrm{mi}$ & 0.23 & 0.041 & 38 \\
\hline Lower LI Cloudshine & $1.5-2 \mathrm{mi}$ & 0.20 & 0.041 & 31 \\
\hline Lower LI Groundshine & $0-0.25 \mathrm{mi}$ & 11.2 & 0.0073 & 2250 \\
\hline Lower LI Groundshine & $0.25-0.33 \mathrm{mi}$ & 5.85 & 0.0066 & 1170 \\
\hline Lower LI Groundshine & $0.33-0.4 \mathrm{mi}$ & 4.8 & 0.0064 & 959 \\
\hline Lower LI Groundshine & $0.4-0.5 \mathrm{mi}$ & 3.97 & 0.0061 & 793 \\
\hline Lower LI Groundshine & $0.5-1.25 \mathrm{mi}$ & 2.02 & 0.0047 & 403 \\
\hline Lower LI Groundshine & $1.25-1.33 \mathrm{mi}$ & 1.26 & 0.0036 & 250 \\
\hline Lower LI Groundshine & $1.22-1.4 \mathrm{mi}$ & 1.17 & 0.0035 & 233 \\
\hline Lower LI Groundshine & $1.4-1.5 \mathrm{mi}$ & 1.08 & 0.0033 & 216 \\
\hline Lower LI Groundshine & $1.5-2 \mathrm{mi}$ & 0.8 & 0.0027 & 160 \\
\hline Lower LI Inhalation Acute & $0-0.25 \mathrm{mi}$ & 20.3 & 0.52 & 3960 \\
\hline Lower LI Inhalation Acute & $0.25-0.33 \mathrm{mi}$ & 10.8 & 0.48 & 2060 \\
\hline Lower LI Inhalation Acute & $0.33-0.4 \mathrm{mi}$ & 8.92 & 0.46 & 1690 \\
\hline Lower LI Inhalation Acute & $0.4-0.5 \mathrm{mi}$ & 7.44 & 0.44 & 1400 \\
\hline Lower LI Inhalation Acute & $0.5-1.25 \mathrm{mi}$ & 3.93 & 0.36 & 714 \\
\hline Lower LI Inhalation Acute & $1.25-1.33 \mathrm{mi}$ & 2.51 & 0.30 & 442 \\
\hline Lower LI Inhalation Acute & $1.22-1.4 \mathrm{mi}$ & 2.35 & 0.30 & 412 \\
\hline
\end{tabular}


Table B.2.3 (continued)

Appendix B Auxiliary Dose Information

\begin{tabular}{||l|c|c|c|c||}
\hline \multicolumn{1}{|c|}{ Organ/Pathway } & Distance & $\begin{array}{c}\text { Dose to } \\
\text { Average } \\
\text { Population }\end{array}$ & $\begin{array}{c}\text { Dose to } \\
\text { Evacuee } \\
\text { Population }\end{array}$ & $\begin{array}{c}\text { Dose to Non- } \\
\text { Evacuee } \\
\text { Population }\end{array}$ \\
\hline Lower LI Inhalation Acute & $1.4-1.5 \mathrm{mi}$ & 2.19 & 0.29 & 381 \\
\hline Lower LI Inhalation Acute & $1.5-2 \mathrm{mi}$ & 1.67 & 0.27 & 281 \\
\hline Lower LI Resuspension Acute & $0-0.25 \mathrm{mi}$ & 0.56 & 0.0004 & 111 \\
\hline Lower LI Resuspension Acute & $0.25-0.33 \mathrm{mi}$ & 0.29 & 0.00036 & 58 \\
\hline Lower LI Resuspension Acute & $0.33-0.4 \mathrm{mi}$ & 0.24 & 0.00035 & 47 \\
\hline Lower LI Resuspension Acute & $0.4-0.5 \mathrm{mi}$ & 0.20 & 0.00033 & 39 \\
\hline Lower LI Resuspension Acute & $0.5-1.25 \mathrm{mi}$ & 0.10 & 0.00026 & 20 \\
\hline Lower LI Resuspension Acute & $1.25-1.33 \mathrm{mi}$ & 0.062 & 0.00019 & 12 \\
\hline Lower LI Resuspension Acute & $1.22-1.4 \mathrm{mi}$ & 0.058 & 0.00018 & 12 \\
\hline Lower LI Resuspension Acute & $1.41 .5 \mathrm{mi}$ & 0.054 & 0.00017 & 11 \\
\hline Lower LI Resuspension Acute & $1.5-2 \mathrm{mi}$ & 0.04 & 0.00013 & 7.9 \\
\hline Lower LI Total Acute & $0-0.25 \mathrm{mi}$ & 32.9 & 0.54 & 6470 \\
\hline Lower LI Total Acute & $0.25-0.33 \mathrm{mi}$ & 17.5 & 0.51 & 3400 \\
\hline Lower LI Total Acute & $0.33-0.4 \mathrm{mi}$ & 14.5 & 0.50 & 2800 \\
\hline Lower LI Total Acute & $0.4-0.5 \mathrm{mi}$ & 12.1 & 0.48 & 2320 \\
\hline Lower LI Total Acute & $0.5-1.25 \mathrm{mi}$ & 6.37 & 0.41 & 1190 \\
\hline Lower LI Total Acute & $1.25-1.33 \mathrm{mi}$ & 4.08 & 0.35 & 747 \\
\hline Lower LI Total Acute & $1.22-1.4 \mathrm{mi}$ & 3.82 & 0.34 & 696 \\
\hline Lower LI Total Acute & $1.4-1.5 \mathrm{mi}$ & 3.56 & 0.34 & 645 \\
\hline Lower LI Total Acute & $1.5-2 \mathrm{mi}$ & 2.71 & 0.31 & 480 \\
\hline
\end{tabular}


Appendix B Auxiliary Dose Information

Table B.2.4

Fraction of the centerline dose to the Red Marrow as a function of distance and by pathway for the total population, the evacuees and the non-evacuees. The evacuees followed the Mean Evacuation and the Source Term was MPB1.

\begin{tabular}{|c|c|c|c|c|}
\hline Total Population & Cloudshine & Groundshine & Inhalation Acute & Resuspension Acute \\
\hline $0-0.25 \mathrm{mi}$ & 0.05 & 0.72 & 0.23 & 0.01 \\
\hline $0.25-0.33 \mathrm{mi}$ & 0.07 & 0.70 & 0.23 & 0.01 \\
\hline $0.33-0.4 \mathrm{mi}$ & 0.07 & 0.69 & 0.23 & 0.01 \\
\hline $0.4-0.5 \mathrm{mi}$ & 0.08 & 0.69 & 0.23 & 0.01 \\
\hline $0.5-1.25 \mathrm{mi}$ & 0.11 & 0.66 & 0.23 & 0.01 \\
\hline $1.25-1.33 \mathrm{mi}$ & 0.13 & 0.64 & 0.22 & 0.01 \\
\hline $1.33-1.4 \mathrm{mi}$ & 0.13 & 0.64 & 0.22 & 0.01 \\
\hline $1.4-1.5 \mathrm{mi}$ & 0.13 & 0.64 & 0.22 & 0.01 \\
\hline $1.5-2 \mathrm{mi}$ & 0.15 & 0.62 & 0.22 & 0.01 \\
\hline Evacuee Population & Cloudshine & Groundshine & Inhalation Acute & Resuspension Acute \\
\hline $0-0.25 \mathrm{mi}$ & 0.20 & 0.07 & 0.73 & 0 \\
\hline $0.25-0.33 \mathrm{mi}$ & 0.28 & 0.06 & 0.66 & 0 \\
\hline $0.33-0.4 \mathrm{mi}$ & 0.30 & 0.06 & 0.63 & 0 \\
\hline $0.4-0.5 \mathrm{mi}$ & 0.33 & 0.06 & 0.61 & 0 \\
\hline $0.5-1.25 \mathrm{mi}$ & 0.40 & 0.05 & 0.55 & 0 \\
\hline $1.25-1.33 \mathrm{mi}$ & 0.46 & 0.04 & 0.5 & 0 \\
\hline $1.33-1.4 \mathrm{mi}$ & 0.47 & 0.04 & 0.49 & 0 \\
\hline $1.4-1.5 \mathrm{mi}$ & 0.47 & 0.04 & 0.49 & 0 \\
\hline $1.5-2 \mathrm{mi}$ & 0.50 & 0.03 & 0.47 & 0 \\
\hline Non-Evacuee Population & Cloudshine & Groundshine & Inhalation Acute & Resuspension Acute \\
\hline $0-0.25 \mathrm{mi}$ & 0.05 & 0.72 & 0.23 & 0.01 \\
\hline $0.25-0.33 \mathrm{mi}$ & 0.07 & 0.71 & 0.22 & 0.01 \\
\hline $0.33-0.4 \mathrm{mi}$ & 0.07 & 0.70 & 0.22 & 0.01 \\
\hline $0.4-0.5 \mathrm{mi}$ & 0.08 & 0.70 & 0.22 & 0.01 \\
\hline $0.5-1.25 \mathrm{mi}$ & 0.10 & 0.68 & 0.22 & 0.01 \\
\hline $1.25-1.33 \mathrm{mi}$ & 0.11 & 0.67 & 0.21 & 0.01 \\
\hline $1.33-1.4 \mathrm{mi}$ & 0.11 & 0.67 & 0.21 & 0.01 \\
\hline $1.4-1.5 \mathrm{mi}$ & 0.12 & 0.67 & 0.21 & 0.01 \\
\hline $1.5-2 \mathrm{mi}$ & 0.13 & 0.66 & 0.21 & 0.01 \\
\hline
\end{tabular}


Table B.2.5

Fraction of the centerline dose to the Lungs as a function of distance and by pathway for the total population, the evacuees and the non-evacuees. The evacuees followed the Mean Evacuation and the source term was MPB1.

\begin{tabular}{|c|c|c|c|c|}
\hline Total Population & Cloudshine & Groundshine & Inhalation Acute & Resuspension Acute \\
\hline $0-0.25 \mathrm{mi}$ & 0.03 & 0.36 & 0.60 & 0.02 \\
\hline $0.25-0.33 \mathrm{mi}$ & 0.03 & 0.35 & 0.60 & 0.02 \\
\hline $0.33-0.4 \mathrm{mi}$ & 0.04 & 0.35 & 0.60 & 0.02 \\
\hline $0.4-0.5 \mathrm{mi}$ & 0.04 & 0.34 & 0.60 & 0.02 \\
\hline $0.5-1.25 \mathrm{mi}$ & 0.05 & 0.33 & 0.60 & 0.02 \\
\hline $1.25-1.33 \mathrm{mi}$ & 0.06 & 0.32 & 0.60 & 0.01 \\
\hline $1.33-1.4 \mathrm{mi}$ & 0.07 & 0.32 & 0.60 & 0.01 \\
\hline $1.4-1.5 \mathrm{mi}$ & 0.07 & 0.32 & 0.60 & 0.01 \\
\hline $1.5-2 \mathrm{mi}$ & 0.08 & 0.31 & 0.60 & 0.01 \\
\hline Evacuee Population & Cloudshine & Groundshine & Inhalation Acute & Resuspension Acute \\
\hline $0-0.25 \mathrm{mi}$ & 0.04 & 0.01 & 0.95 & 0 \\
\hline $0.25-0.33 \mathrm{mi}$ & 0.06 & 0.01 & 0.93 & 0 \\
\hline $0.33-0.4 \mathrm{mi}$ & 0.07 & 0.01 & 0.92 & 0 \\
\hline $0.4-0.5 \mathrm{mi}$ & 0.07 & 0.01 & 0.92 & 0 \\
\hline $0.5-1.25 \mathrm{mi}$ & 0.10 & 0.01 & 0.89 & 0 \\
\hline $1.25-1.33 \mathrm{mi}$ & 0.12 & 0.01 & 0.87 & 0 \\
\hline $1.33-1.4 \mathrm{mi}$ & 0.12 & 0.01 & 0.87 & 0 \\
\hline $1.4-1.5 \mathrm{mi}$ & 0.12 & 0.01 & 0.87 & 0 \\
\hline $1.5-2 \mathrm{mi}$ & 0.13 & 0.01 & 0.86 & 0 \\
\hline Non-Evacuee Population & Cloudshine & Groundshine & Inhalation Acute & Resuspension Acute \\
\hline $0-0.25 \mathrm{mi}$ & 0.02 & 0.36 & 0.59 & 0.02 \\
\hline $0.25-0.33 \mathrm{mi}$ & 0.03 & 0.36 & 0.59 & 0.02 \\
\hline $0.33-0.4 \mathrm{mi}$ & 0.04 & 0.36 & 0.59 & 0.02 \\
\hline $0.4-0.5 \mathrm{mi}$ & 0.04 & 0.36 & 0.59 & 0.02 \\
\hline $0.5-1.25 \mathrm{mi}$ & 0.05 & 0.35 & 0.58 & 0.02 \\
\hline $1.25-1.33 \mathrm{mi}$ & 0.06 & 0.35 & 0.57 & 0.02 \\
\hline $1.33-1.4 \mathrm{mi}$ & 0.06 & 0.35 & 0.57 & 0.02 \\
\hline $1.4-1.5 \mathrm{mi}$ & 0.06 & 0.35 & 0.57 & 0.02 \\
\hline $1.5-2 \mathrm{mi}$ & 0.07 & 0.35 & 0.57 & 0.02 \\
\hline
\end{tabular}

*Zero dose at this distance 
Appendix B Auxiliary Dose Information

Table B.2.6

Fraction of the centerline dose(REM) to the Lower Large Intestine as a function of distance and by pathway for the total population and for each cohort. The evacuees followed the Mean EP and the source term was MPB1.

\begin{tabular}{|c|c|c|c|c|}
\hline Total Population & Cloudshine & Groundshine & Inhalation Acute & Resuspension Acute \\
\hline $0-0.25 \mathrm{mi}$ & 0.024 & 0.34 & 0.62 & 0.017 \\
\hline $0.25-0.33 \mathrm{mi}$ & 0.033 & 0.33 & 0.62 & 0.017 \\
\hline $0.33-0.4 \mathrm{mi}$ & 0.036 & 0.33 & 0.62 & 0.016 \\
\hline $0.4-0.5 \mathrm{mi}$ & 0.039 & 0.33 & 0.62 & 0.016 \\
\hline $0.5-1.25 \mathrm{mi}$ & 0.051 & 0.32 & 0.62 & 0.016 \\
\hline $1.25-1.33 \mathrm{mi}$ & 0.061 & 0.31 & 0.62 & 0.015 \\
\hline $1.22-1.4 \mathrm{mi}$ & 0.063 & 0.31 & 0.62 & 0.015 \\
\hline $1.4-1.5 \mathrm{mi}$ & 0.065 & 0.30 & 0.62 & 0.015 \\
\hline $1.5-2 \mathrm{mi}$ & 0.072 & 0.30 & 0.62 & 0.015 \\
\hline Evacuee Population & Cloudshine & Groundshine & Inhalation Acute & Resuspension Acute \\
\hline $0-0.25 \mathrm{mi}$ & 0.039 & 0.013 & 0.95 & 0.001 \\
\hline $0.25-0.33 \mathrm{mi}$ & 0.058 & 0.013 & 0.93 & 0.001 \\
\hline $0.33-0.4 \mathrm{mi}$ & 0.066 & 0.013 & 0.92 & 0.001 \\
\hline $0.4-0.5 \mathrm{mi}$ & 0.072 & 0.013 & 0.92 & 0.001 \\
\hline $0.5-1.25 \mathrm{mi}$ & 0.097 & 0.012 & 0.89 & 0.001 \\
\hline $1.25-1.33 \mathrm{mi}$ & 0.118 & 0.01 & 0.87 & 0.001 \\
\hline $1.22-1.4 \mathrm{mi}$ & 0.12 & 0.01 & 0.87 & 0.001 \\
\hline $1.4-1.5 \mathrm{mi}$ & 0.123 & 0.01 & 0.87 & 0.001 \\
\hline $1.5-2 \mathrm{mi}$ & 0.132 & 0.009 & 0.86 & 0 \\
\hline Non-Evacuee Population & Cloudshine & Groundshine & Inhalation Acute & Resuspension Acute \\
\hline $0-0.25 \mathrm{mi}$ & 0.024 & 0.35 & 0.61 & 0.017 \\
\hline $0.25-0.33 \mathrm{mi}$ & 0.032 & 0.34 & 0.60 & 0.017 \\
\hline $0.33-0.4 \mathrm{mi}$ & 0.035 & 0.34 & 0.60 & 0.017 \\
\hline $0.4-0.5 \mathrm{mi}$ & 0.037 & 0.34 & 0.60 & 0.017 \\
\hline $0.5-1.25 \mathrm{mi}$ & 0.048 & 0.34 & 0.60 & 0.017 \\
\hline $1.25-1.33 \mathrm{mi}$ & 0.056 & 0.34 & 0.59 & 0.017 \\
\hline $1.22-1.4 \mathrm{mi}$ & 0.057 & 0.34 & 0.59 & 0.017 \\
\hline $1.4-1.5 \mathrm{mi}$ & 0.059 & 0.34 & 0.59 & 0.017 \\
\hline $1.5-2 \mathrm{mi}$ & 0.064 & 0.33 & 0.59 & 0.017 \\
\hline
\end{tabular}

*Zero dose at this distance 
Table B.3.1

Centerline dose (Rem) to the Red Marrow as a function of distance by pathway for source term MPB3. The third column is the dose to the average (weighted) population, the fourth column is the dose to the evacuees following the Conservative Evacuation, and the last column is for the dose to the non-evacuees.

\begin{tabular}{|c|c|c|c|c|}
\hline Organ/Pathway & Distance & $\begin{array}{c}\text { Dose to } \\
\text { Average } \\
\text { Population }\end{array}$ & $\begin{array}{c}\text { Dose to } \\
\text { Evacuee } \\
\text { Population }\end{array}$ & $\begin{array}{l}\text { Dose to Non- } \\
\text { Evacuee } \\
\text { Population }\end{array}$ \\
\hline Red Marruw Cloudshine & $0-0.25 \mathrm{mi}$ & 98.6 & 98.5 & 101 \\
\hline Red Marrow Cloudshine & $0.25-0.33 \mathrm{mi}$ & 72.2 & 72.1 & 73.9 \\
\hline Red Marrow Cloudshine & $0.33-0.4 \mathrm{mi}$ & 64.8 & 64.8 & 66.3 \\
\hline Red Marrow Cloudshine & $0.4-0.5 \mathrm{mi}$ & 58.2 & 58.2 & 59.4 \\
\hline Red Marrow Cloudshine & $0.5-1.25 \mathrm{mi}$ & 39.5 & 39.5 & 40 \\
\hline Red Marrow Cloudshine & $1.25-1.33 \mathrm{mi}$ & 28.5 & 28.5 & 28.7 \\
\hline Red Marrow Cloudshine & $1.33-1.4 \mathrm{mi}$ & 27.2 & 27.2 & 27.4 \\
\hline Red Marrow Cloudshine & $1.4-1.5 \mathrm{mi}$ & 25.9 & 25.9 & 26.1 \\
\hline Red Marrow Cloudshine & $1.5-2 \mathrm{mi}$ & 21.7 & 21.7 & 21.7 \\
\hline Red Marrow Groundshine & $0-0.25 \mathrm{mi}$ & 114 & 100 & 365 \\
\hline Red Marrow Groundshine & $0.25-0.33 \mathrm{mi}$ & 61.3 & 54.6 & 189 \\
\hline Red Marrow Groundshine & $0.33-0.4 \mathrm{mi}$ & 50.8 & 45.4 & 155 \\
\hline Red Marrow Groundshine & $0.4-0.5 \mathrm{mi}$ & 42.5 & 38 & 128 \\
\hline Red Marrow Groundshine & $0.5-1.25 \mathrm{mi}$ & 22.5 & 20.4 & 62.6 \\
\hline Red Marrow Groundshine & $1.25-1.33 \mathrm{mi}$ & 13.5 & 12.3 & 35.5 \\
\hline Red Marrow Groundshine & $1.33-1.4 \mathrm{mi}$ & 12.6 & 11.6 & 33.1 \\
\hline Red Marrow Groundshine & $1.4-1.5 \mathrm{mi}$ & 11.8 & 10.8 & 30.6 \\
\hline Red Marrow Groundshine & $1.5-2 \mathrm{mi}$ & 9.18 & 8.47 & 22.7 \\
\hline Red Marrow Inhalation Acute & $0-0.25 \mathrm{mi}$ & 156 & 156 & 160 \\
\hline Red Marrow Inhalation Acute & $0.25-0.33 \mathrm{mi}$ & 83.1 & 83.1 & 83 \\
\hline Red Marrow Inhalation Acute & $0.33-0.4 \mathrm{mi}$ & 68.5 & 68.5 & 67.9 \\
\hline Red Marrow Inhalation Acute & $0.4-0.5 \mathrm{mi}$ & 56.9 & 56.9 & 55.9 \\
\hline Red Marrow Inhalation Acute & $0.5-1.25 \mathrm{mi}$ & 29.3 & 29.4 & 27.4 \\
\hline
\end{tabular}


Appendix B Auxiliary Dose Information

Table B.3.1 (continued)

\begin{tabular}{|c|c|c|c|c|}
\hline Organ/Pathway & Distance & $\begin{array}{c}\text { Dose to } \\
\text { Average } \\
\text { Population }\end{array}$ & $\begin{array}{c}\text { Dose to } \\
\text { Evacuee } \\
\text { Population }\end{array}$ & $\begin{array}{c}\text { Dose to Non- } \\
\text { Evacuee } \\
\text { Population }\end{array}$ \\
\hline Red Marrow Inhalation Acute & $1.25-1.33 \mathrm{mi}$ & 16.8 & 16.9 & 15.4 \\
\hline Red Marrow Inhalation Acute & $1.33-1.4 \mathrm{mi}$ & 15.7 & 15.7 & 14.4 \\
\hline Red Marrow Inhalation Acute & 1.4-1.5 mi & 14.6 & 14.6 & 13.3 \\
\hline Red Marrow Inhalation Acute & $1.5-2 \mathrm{mi}$ & 11.1 & 11.2 & 9.81 \\
\hline Red Marrow Resuspension Acute & $0-0.25 \mathrm{mi}$ & 0.293 & 0.139 & $3.2 ?$ \\
\hline Red Marrow Resuspension Acute & $0.25-0.33 \mathrm{mi}$ & 0.178 & 0.0998 & 1.67 \\
\hline Red Marrow Resuspension Acute & $0.33-0.4 \mathrm{mi}$ & 0.155 & 0.0909 & 1.37 \\
\hline Red Marrow Resuspension Acute & $0.4-0.5 \mathrm{mi}$ & 0.135 & 0.0833 & 1.13 \\
\hline Red Marrow Resuspension Acute & $0.5-1.25 \mathrm{mi}$ & 0.0858 & 0.0611 & 0.555 \\
\hline Red Marrow Resuspension Acute & $1.25-1.33 \mathrm{mi}$ & 0.0615 & 0.0481 & 0.315 \\
\hline Red Marrow Resuspension Acute & $1.33-1.4 \mathrm{mi}$ & 0.0587 & 0.0463 & 0.293 \\
\hline Red Marrow Resuspension Acute & $1.4-1.5 \mathrm{mi}$ & 0.0558 & 0.0444 & 0.271 \\
\hline Red Marrow Resuspension Acute & $1.5-2 \mathrm{mi}$ & 0.0469 & 0.0387 & 0.202 \\
\hline Red Marrow Total Acute & $0-0.25 \mathrm{mi}$ & 369 & 355 & 630 \\
\hline Red Marrow Total Acute & $0.25-0.33 \mathrm{mi}$ & 217 & 210 & 348 \\
\hline Red Marrow Total Acute & $0.33-0.4 \mathrm{mi}$ & 184 & 179 & 290 \\
\hline Red Marrow Total Acute & $0.4-0.5 \mathrm{mi}$ & 158 & 153 & 244 \\
\hline Red Marrow Total Acute & $0.5-1.25 \mathrm{mi}$ & 91.4 & 89.3 & 131 \\
\hline Red Marrow Total Acute & $1.25-1.33 \mathrm{mi}$ & 58.8 & 57.7 & 80 \\
\hline Red Marrow Total Acute & $1.33-1.4 \mathrm{mi}$ & 55.6 & 54.6 & 75.1 \\
\hline Red Marrow Total Acute & $1.4-1.5 \mathrm{mi}$ & 52.3 & 51.4 & 70.2 \\
\hline Red Marrow Total Acute & $1.5-2 \mathrm{mi}$ & 42 & 41.4 & 54.4 \\
\hline
\end{tabular}


Table B.3.2

Centerline dose (Rem) to the Lungs as a function of distance by pathway for source term MPB3. The third column is the dose to the average (weighted) population, the fourth column is the dose to the evacuees following the Conservative Evacuation, and the last column is for the dose to the non-evacuees.

\begin{tabular}{|c|c|c|c|c|}
\hline Organ/Pathway & Distance & $\begin{array}{c}\text { Dose to } \\
\text { Average } \\
\text { Population }\end{array}$ & $\begin{array}{c}\text { Dose to } \\
\text { Evacuee } \\
\text { Population }\end{array}$ & $\begin{array}{c}\text { Dose to Non- } \\
\text { Evacuee } \\
\text { Population }\end{array}$ \\
\hline Lungs Cloudshine & $0-0.25 \mathrm{mi}$ & 99.5 & 99.4 & 102 \\
\hline Lungs Cloudshine & $0.25-0.33 \mathrm{mi}$ & 72.9 & 72.8 & 74.6 \\
\hline Lungs Cloudshine & $0.33-0.4 \mathrm{mi}$ & 65.5 & 65.4 & 66.9 \\
\hline Lungs Cloudshine & $0.4-0.5 \mathrm{mi}$ & 58.8 & 58.7 & 60 \\
\hline Lungs Cloudshine & $0.5-1.25 \mathrm{mi}$ & 40 & 39.9 & 40.4 \\
\hline Lungs Cloudshine & $1.25-1.33 \mathrm{mi}$ & 28.8 & 28.8 & 29 \\
\hline Lungs Cloudshine & $1.33-1.4 \mathrm{mi}$ & 27.5 & 27.5 & 27.7 \\
\hline Lungs Cloudshine & $1.4-1.5 \mathrm{mi}$ & 26.2 & 26.2 & 26.4 \\
\hline Lungs Cloudshine & $1.5-2 \mathrm{mi}$ & 21.9 & 21.9 & 22 \\
\hline Lungs Groundshine & $0-0.25 \mathrm{mi}$ & 112 & 99.3 & 361 \\
\hline Lungs Groundshine & $0.25-0.33 \mathrm{mi}$ & 60.6 & 54 & 187 \\
\hline Lungs Groundshine & $0.33-0.4 \mathrm{mi}$ & 50.2 & 44.8 & 153 \\
\hline Lungs Groundshine & $0.4-0.5 \mathrm{mi}$ & 42 & 37.5 & 126 \\
\hline Lungs Groundshine & $0.5-1.25 \mathrm{mi}$ & 22.2 & 20.1 & 61.8 \\
\hline Lungs Groundshine & $1.25-1.33 \mathrm{mi}$ & 13.3 & 12.2 & 35.1 \\
\hline Lungs Groundshine & $1.33-1.4 \mathrm{mi}$ & 12.5 & 11.4 & 32.7 \\
\hline Lungs Groundshine & $1.4-1.5 \mathrm{mi}$ & 11.6 & 10.7 & 30.2 \\
\hline Lungs Groundshine & $1.5-2 \mathrm{mi}$ & 9.08 & 8.37 & 22.5 \\
\hline Lungs Inhalation Acute & $0-0.25 \mathrm{mi}$ & 820 & 819 & 839 \\
\hline Lungs Inhalation Acvite & $0.25-0.33 \mathrm{mi}$ & 436 & 436 & 436 \\
\hline Lungs Inhalation Acute & $0.33-0.4 \mathrm{mi}$ & 359 & 360 & 356 \\
\hline Lungs Inhalation Acute & $0.4-0.5 \mathrm{mi}$ & 298 & 299 & 293 \\
\hline Lungs Inhalation Acute & $0.5-1.25 \mathrm{mi}$ & $: 53$ & 154 & 144 \\
\hline Lungs Inhalation Acute & $1.25-1.33 \mathrm{mi}$ & 88 & 88.4 & 80.9 \\
\hline Lungs Inhalation Acute & $1.33-1.4 \mathrm{mi}$ & 82.1 & 82.4 & 75.2 \\
\hline
\end{tabular}


Appendix B Auxiliary Dose Information

Table B.3.2 (continued)

\begin{tabular}{|c|c|c|c|c|}
\hline Organ/Pathway & Distance & $\begin{array}{c}\text { Dose to } \\
\text { Average } \\
\text { Population }\end{array}$ & $\begin{array}{c}\text { Dose to } \\
\text { Evacuee } \\
\text { Population }\end{array}$ & $\begin{array}{c}\text { Dose to Non- } \\
\text { Evacuee } \\
\text { Population }\end{array}$ \\
\hline Lungs Inhalation Acute & $1.4-1.5 \mathrm{mi}$ & 76.1 & 76.5 & 69.5 \\
\hline Lungs Inhalation Acute & $1.5-2 \mathrm{mi}$ & 58.1 & 58.4 & 51.4 \\
\hline Lungs Resuspension Acute & $0-0.25 \mathrm{mi}$ & 1.57 & 0.759 & 17 \\
\hline Lungs Resuspension Acute & $0.25-0.33 \mathrm{mi}$ & 0.953 & 0.539 & 8.81 \\
\hline Lungs Resuspension Acute & $0.33-0.4 \mathrm{mi}$ & 0.825 & 0.49 & 7.2 \\
\hline Lungs Resuspension Acute & $0.4-0.5 \mathrm{mi}$ & 0.722 & 0.447 & 5.94 \\
\hline Lungs Resuspension Acute & $0.5-1.25 \mathrm{mi}$ & 0.455 & 0.325 & 2.92 \\
\hline Lungs Resuspension Acute & $1.25-1.33 \mathrm{mi}$ & 0.325 & 0.255 & 1.66 \\
\hline Lungs Resuspension Acute & $1.33-1.4 \mathrm{mi}$ & 0.31 & 0.245 & 1.54 \\
\hline Lungs Resuspension Acute & $1.4-1.5 \mathrm{mi}$ & 0.295 & 0.235 & 1.43 \\
\hline Lungs Resuspension Acute & $1.5-2 \mathrm{mi}$ & 0.247 & 0.204 & 1.06 \\
\hline Lungs Total Acute & $0-0.25 \mathrm{mi}$ & 1030 & 1020 & 1320 \\
\hline Lungs Total Acute & $0.25-0.33 \mathrm{mi}$ & 571 & 564 & 706 \\
\hline Lungs Total Acute & $0.33-0.4 \mathrm{mi}$ & 476 & 470 & 583 \\
\hline Lungs Total Acute & $0.4-0.5 \mathrm{mi}$ & 400 & 395 & 485 \\
\hline Lungs Total Acute & $0.5-1.25 \mathrm{mi}$ & 216 & 214 & 249 \\
\hline Lungs Total Acute & $1.25-1.33 \mathrm{mi}$ & 130 & 130 & 147 \\
\hline Lungs Total Acute & $1.33-1.4 \mathrm{mi}$ & 122 & 122 & 137 \\
\hline Lungs Total Acute & 1.4-1.5 mi & 114 & 114 & 128 \\
\hline Lungs Total Acute & 1.5-2 mi & 89.4 & 89 & 96.8 \\
\hline
\end{tabular}


Table B.3.3

Centerline dose (Rem) to the Lower Large Intestine as a function of distance by pathway for source term MPB3. The third column is the dose to the average (weighted) population, the fourth column is the dose to the evacuees following the Conservative Evacuation, and the last column is for the dose to the non-evacuees.

\begin{tabular}{|c|c|c|c|c|}
\hline Organ/Pathway & Distance & $\begin{array}{c}\text { Dose to } \\
\text { Average } \\
\text { Population }\end{array}$ & $\begin{array}{c}\text { Dose to } \\
\text { Evacuee } \\
\text { Population }\end{array}$ & $\begin{array}{c}\text { Dose to Non- } \\
\text { Evacuee } \\
\text { Population }\end{array}$ \\
\hline Lower LI Cloudshine & $0-0.25 \mathrm{mi}$ & 90.6 & 90.5 & 93.2 \\
\hline Lower LI Cloudshine & $0.25-0.33 \mathrm{mi}$ & 66.3 & 66.2 & 67.9 \\
\hline Lower LI Cloudshine & $0.33-0.4 \mathrm{mi}$ & 59.6 & 59.5 & 60.9 \\
\hline Lower LI Cloudshine & $0.4-0.5 \mathrm{mi}$ & 53.5 & 53.5 & 54.6 \\
\hline Lower LI Cloudshine & $0.5-1.25 \mathrm{mi}$ & 36.4 & 36.4 & 36.8 \\
\hline Lower LI Cloudshine & $1.25-1.33 \mathrm{mi}$ & 26.2 & 26.2 & 26.4 \\
\hline Lower LI Cloudshine & $1.33-1.4 \mathrm{mi}$ & 25.1 & 25 & 25.2 \\
\hline Lower LI Cloudshine & $1.4-1.5 \mathrm{mi}$ & 23.9 & 23.9 & 24 \\
\hline Lower LI Cloudshine & $1.5-2 \mathrm{mi}$ & 20 & 20 & 20 \\
\hline Lower LI Groundshine & $0-0.25 \mathrm{mi}$ & 102 & 89.9 & 327 \\
\hline Lower LI Groundshine & $0.25-0.33 \mathrm{mi}$ & 54.9 & 48.8 & 170 \\
\hline Lower LI Groundshine & $0.33-0.4 \mathrm{mi}$ & 45.5 & 40.6 & 139 \\
\hline Lower LI Groundshine & $0.4-0.5 \mathrm{mi}$ & 38 & 34 & 114 \\
\hline Lower LI Groundshine & $0.5-1.25 \mathrm{mi}$ & 20.1 & 18.2 & 56.1 \\
\hline Lower LI Groundshine & $1.25-1.33 \mathrm{mi}$ & 12.1 & 11 & 31.9 \\
\hline Lower LI Groundshine & $1.33-1.4 \mathrm{mi}$ & 11.3 & 10.3 & 29.6 \\
\hline Lower LI Groundshine & $1.4-1.5 \mathrm{mi}$ & 10.5 & 9.66 & 27.4 \\
\hline Lower LI Groundshine & $1.5-2 \mathrm{mi}$ & 8.22 & 7.58 & 20.4 \\
\hline Lower LI Inhalation Acute & $0-0.25 \mathrm{mi}$ & 856 & 855 & 876 \\
\hline Lower LI Inhalation Acute & $0.25-0.33 \mathrm{mi}$ & 455 & 455 & 455 \\
\hline Lower LI Inhalation Acute & $0.33-0.4 \mathrm{mi}$ & 375 & 376 & 372 \\
\hline Lower LI Inhalation Acute & $0.4-0.5 \mathrm{mi}$ & 312 & 312 & 306 \\
\hline Lower LI Inhalation Acute & $0.5-1.25 \mathrm{mi}$ & 160 & 161 & 150 \\
\hline Lower LI Inhalation Acute & $1.25-1.33 \mathrm{mi}$ & 92 & 92.4 & 84.5 \\
\hline Lower LI Inhalation Acute & $1.33-1.4 \mathrm{mi}$ & 85.8 & 86.2 & 78.5 \\
\hline
\end{tabular}


Appendix B Auxiliary Dose Information

Table B.3.3 (continued)

\begin{tabular}{||l|l|c|c|c|}
\hline \multicolumn{1}{|c|}{ Organ/Pathway } & \multicolumn{1}{|c|}{ Distance } & $\begin{array}{c}\text { Dose to } \\
\text { Average } \\
\text { Population }\end{array}$ & $\begin{array}{c}\text { Dose to } \\
\text { Evacuee } \\
\text { Population }\end{array}$ & $\begin{array}{c}\text { Dose to Non- } \\
\text { Evacuee } \\
\text { Population }\end{array}$ \\
\hline Lower LI Inhalation Acute & $1.4-1.5 \mathrm{mi}$ & 79.7 & 80 & 72.6 \\
\hline Lower LI Inhalation Acute & $1.5-2 \mathrm{mi}$ & 60.8 & 61.2 & 53.6 \\
\hline Lower LI Resuspension Acute & $0-0.25 \mathrm{mi}$ & 1.58 & 0.73 & 17.7 \\
\hline Lower LI Resuspension Acute & $0.25-0.33 \mathrm{mi}$ & 0.965 & 0.531 & 9.2 \\
\hline Lower LI Resuspension Acute & $0.33-0.4 \mathrm{mi}$ & 0.838 & 0.486 & 7.52 \\
\hline Lower LI Resuspension Acute & $0.4-0.5 \mathrm{mi}$ & 0.734 & 0.446 & 6.2 \\
\hline Lower LI Resuspension Acute & $0.5-1.25 \mathrm{mi}$ & 0.466 & 0.33 & 3.05 \\
\hline Lower LI Resuspension Acute & $1.25-1.33 \mathrm{mi}$ & 0.335 & 0.261 & 1.73 \\
\hline Lower LI Resuspension Acute & $1.33-1.4 \mathrm{mi}$ & 0.32 & 0.252 & 1.61 \\
\hline Lower LI Resuspension Acute & $1.4-1.5 \mathrm{mi}$ & 0.304 & 0.241 & 1.49 \\
\hline Lower LI Resuspension Acute & $1.5-2 \mathrm{mi}$ & 0.256 & 0.211 & 1.11 \\
\hline Lower LI Total Acute & $0-0.25 \mathrm{mi}$ & 1050 & 1040 & 1310 \\
\hline Lower LI Total Acute & $0.25-0.33 \mathrm{mi}$ & 578 & 571 & 702 \\
\hline Lower LI Total Acute & $0.33-0.4 \mathrm{mi}$ & 481 & 476 & 579 \\
\hline Lower LI Total Acute & $0.4-0.5 \mathrm{mi}$ & 404 & 400 & 481 \\
\hline Lower LI Total Acute & $0.5-1.25 \mathrm{mi}$ & 217 & 216 & 246 \\
\hline Lower LI Total Acute & $1.25-1.33 \mathrm{mi}$ & 131 & 130 & 144 \\
\hline Lower LI Total Acute & $1.33-1.4 \mathrm{mi}$ & 123 & 122 & 135 \\
\hline Lower LI Total Acute & $1.4-1.5 \mathrm{mi}$ & 114 & 114 & 125 \\
\hline Lower LI Total Acute & $1.5-2 \mathrm{mi}$ & 89.3 & 88.9 & 95.1 \\
\hline
\end{tabular}


Table B.3.4

Fraction of the centerline dose to the Red Marrow as a function of distance and by pathway for the total population (first set of data), the evacuees (second set of data) and the non-evacuees (third set of data). The source term was MPB3 and the evacuees followed the conservative evacuation.

\begin{tabular}{|c|c|c|c|c|}
\hline $\begin{array}{c}\text { Red Marrow/Total } \\
\text { Population }\end{array}$ & Cloudshine & Groundshine & $\begin{array}{c}\text { Inhalation } \\
\text { Acute }\end{array}$ & $\begin{array}{c}\text { Resuspension } \\
\text { Acute }\end{array}$ \\
\hline $0-0.25 \mathrm{mi}$ & 0.27 & 0.31 & 0.42 & 0.0008 \\
\hline $0.25-0.33 \mathrm{mi}$ & 0.33 & 0.28 & 0.38 & 0.0008 \\
\hline $0.33-0.4 \mathrm{mi}$ & 0.35 & 0.28 & 0.37 & 0.0008 \\
\hline $0.4-0.5 \mathrm{mi}$ & 0.37 & 0.27 & 0.36 & 0.0009 \\
\hline $0.5-1.25 \mathrm{mi}$ & 0.43 & 0.25 & 0.32 & 0.0009 \\
\hline $1.25-1.33 \mathrm{mi}$ & 0.48 & 0.23 & 0.29 & 0.001 \\
\hline $1.33-1.4 \mathrm{mi}$ & 0.49 & 0.23 & 0.28 & 0.0011 \\
\hline $1.4-1.5 \mathrm{mi}$ & 0.50 & 0.23 & 0.28 & 0.0011 \\
\hline $1.5-2 \mathrm{mi}$ & 0.52 & 0.22 & 0.26 & 0.0011 \\
\hline $\begin{array}{c}\text { Red Marrow/Evacuee } \\
\text { Population }\end{array}$ & Cloudshine & Groundshine & $\begin{array}{c}\text { Inhalation } \\
\text { Acute }\end{array}$ & $\begin{array}{c}\text { Resuspension } \\
\text { Acute }\end{array}$ \\
\hline $0-0.25 \mathrm{mi}$ & 0.28 & 0.28 & 0.44 & 0.0004 \\
\hline $0.25-0.33 \mathrm{mi}$ & 0.34 & 0.26 & 0.40 & 0.0005 \\
\hline $0.33-0.4 \mathrm{mi}$ & 0.36 & 0.25 & 0.38 & 0.0005 \\
\hline $0.4-0.5 \mathrm{mi}$ & 0.38 & 0.25 & 0.37 & 0.0005 \\
\hline $0.5-1.25 \mathrm{mi}$ & 0.44 & 0.23 & 0.33 & 0.0007 \\
\hline $1.25-1.33 \mathrm{mi}$ & 0.49 & 0.21 & 0.29 & 0.0008 \\
\hline $1.33-1.4 \mathrm{mi}$ & 0.50 & 0.21 & 0.29 & 0.0008 \\
\hline $1.4-1.5 \mathrm{mi}$ & 0.50 & 0.21 & 0.28 & 0.0009 \\
\hline $1.5-2 \mathrm{mi}$ & 0.52 & 0.20 & 0.27 & 0.0009 \\
\hline $\begin{array}{l}\text { Red Marrow/Non- } \\
\text { Evacuee Population }\end{array}$ & Cloudshine & Groundshine & $\begin{array}{c}\text { Inhalation } \\
\text { Acute }\end{array}$ & $\begin{array}{c}\text { Resuspension } \\
\text { Acute }\end{array}$ \\
\hline $0-0.25 \mathrm{mi}$ & 0.16 & 0.58 & 0.25 & 0.0012 \\
\hline $0.25-0.33 \mathrm{mi}$ & 0.21 & 0.54 & 0.24 & 0.0093 \\
\hline 0.33-0.4 mi & 0.23 & 0.53 & 0.23 & 0.0058 \\
\hline $0.4-0.5 \mathrm{mi}$ & 0.24 & 0.52 & 0.23 & 0.0056 \\
\hline
\end{tabular}


Table B.3.4 (continued)

\begin{tabular}{||l|c|c|c|c||}
\hline $\begin{array}{c}\text { Red Marrow/Non- } \\
\text { Evacuee Population }\end{array}$ & Cloudshine & Groundshine & $\begin{array}{c}\text { Inhalation } \\
\text { Acute }\end{array}$ & $\begin{array}{c}\text { Resuspension } \\
\text { Acute }\end{array}$ \\
\hline $0.5-1.25 \mathrm{mi}$ & 0.31 & 0.48 & 0.21 & 0.0086 \\
\hline $1.25-1.33 \mathrm{mi}$ & 0.36 & 0.44 & 0.19 & 0.0069 \\
\hline $1.33-1.4 \mathrm{mi}$ & 0.36 & 0.44 & 0.19 & 0.0042 \\
\hline $1.4-1.5 \mathrm{mi}$ & 0.37 & 0.44 & 0.19 & 0.0042 \\
\hline $1.5-2 \mathrm{mi}$ & 0.40 & 0.42 & 0.18 & 0.005 \\
\hline
\end{tabular}


Table B.3.5

Fraction of the centerline dose to the Lungs as a function of distance and by pathway for the total population (first set of data), the evacuees (second set of data) and the non-evacuees (third set of data). The source term was MPB3 and the evacuees followed the conservative evacuation.

\begin{tabular}{|c|c|c|c|c|}
\hline & Cloudshine & Groundshine & $\begin{array}{c}\text { Inhalation } \\
\text { Acute }\end{array}$ & $\begin{array}{c}\text { Resuspension } \\
\text { Acute }\end{array}$ \\
\hline $0-0.25 \mathrm{mi}$ & 0.10 & 0.11 & 0.80 & 0.0015 \\
\hline $0.25-0.33 \mathrm{mi}$ & 0.13 & 0.11 & 0.76 & 0.0017 \\
\hline $0.33-0.4 \mathrm{mi}$ & 0.14 & 0.11 & 0.75 & 0.0017 \\
\hline $0.4-0.5 \mathrm{mi}$ & 0.15 & 0.11 & 0.75 & 0.0018 \\
\hline $0.5-1.25 \mathrm{mi}$ & 0.19 & 0.10 & 0.71 & 0.0021 \\
\hline $1.25-1.33 \mathrm{mi}$ & 0.22 . & 0.10 & 0.68 & 0.0025 \\
\hline $1.33-1.4 \mathrm{mi}$ & 0.23 & 0.10 & 0.67 & 0.0025 \\
\hline $1.4-1.5 \mathrm{mi}$ & 0.23 & 0.10 & 0.67 & 0.0026 \\
\hline $1.5-2 \mathrm{mi}$ & 0.24 & 0.10 & 0.65 & 0.0028 \\
\hline $\begin{array}{c}\text { Lungs/Evacuee } \\
\text { Population }\end{array}$ & Cloudshine & Groundshine & $\begin{array}{c}\text { Inhalation } \\
\text { Acute }\end{array}$ & $\begin{array}{c}\text { Resuspension } \\
\text { Acute } \\
\end{array}$ \\
\hline $0-0.25 \mathrm{mi}$ & 0.10 & 0.10 & 0.80 & 0.0007 \\
\hline $0.25-0.33 \mathrm{mi}$ & 0.13 & 0.10 & 0.77 & 0.0010 \\
\hline $0.33-0.4 \mathrm{mi}$ & 0.14 & 0.10 & 0.77 & 0.0010 \\
\hline $0.4-0.5 \mathrm{mi}$ & 0.15 & 0.09 & 0.76 & 0.0011 \\
\hline $0.5-1.25 \mathrm{mi}$ & 0.19 & 0.09 & 0.72 & 0.0015 \\
\hline $1.25-1.33 \mathrm{mi}$ & 0.22 & 0.09 & 0.68 & 0.0020 \\
\hline $1.33-1.4 \mathrm{mi}$ & 0.23 & 0.09 & .0 .68 & 0.0020 \\
\hline $1.4-1.5 \mathrm{mi}$ & 0.23 & 0.09 & 0.67 & 0.0021 \\
\hline $1.5-2 \mathrm{mi}$ & 0.25 & 0.09 & 0.66 & 0.0023 \\
\hline $\begin{array}{c}\text { Lungs/Non-Evacuee } \\
\text { Population }\end{array}$ & Cloudshine & Groundshine & $\begin{array}{c}\text { Inhalation } \\
\text { Acute }\end{array}$ & $\begin{array}{c}\text { Resuspension } \\
\text { Acute } \\
\end{array}$ \\
\hline $0-0.25 \mathrm{mi}$ & 0.08 & 0.27 & 0.64 & 0.0129 \\
\hline $0.25-0.33 \mathrm{mi}$ & 0.11 & 0.26 & 0.62 & 0.0125 \\
\hline $0.33-0.4 \mathrm{mi}$ & 0.11 & 0.26 & 0.61 & 0.0123 \\
\hline $0.4-0.5 \mathrm{mi}$ & 0.12 & 0.26 & 0.60 & 0.0122 \\
\hline
\end{tabular}


Table B.3.5 (continued)

\begin{tabular}{||c|c|c|c|c|}
\hline $\begin{array}{c}\text { Lungs/Non-Evacuee } \\
\text { Population }\end{array}$ & Cloudshine & Groundshine & $\begin{array}{c}\text { Inhalation } \\
\text { Acute }\end{array}$ & $\begin{array}{c}\text { Resuspension } \\
\text { Acute }\end{array}$ \\
\hline $0.5-1.25 \mathrm{mi}$ & 0.16 & 0.25 & 0.58 & 0.0117 \\
\hline $1.25-1.33 \mathrm{mi}$ & 0.20 & 0.24 & 0.55 & 0.0113 \\
\hline $1.33-1.4 \mathrm{mi}$ & 0.20 & 0.24 & 0.55 & 0.0112 \\
\hline $1.4-1.5 \mathrm{mi}$ & 0.21 & 0.24 & 0.54 & 0.0112 \\
\hline $1.5-2 \mathrm{mi}$ & 0.23 & 0.23 & 0.53 & 0.0110 \\
\hline
\end{tabular}


Table B.3.6

Fraction of the centerline dose to the Lower Large Intestine as a function of distance and by pathway for the total population (first set of data), the evacuees (second set of data) and the non-evacuees (third set of data). The source term was MPB3 and the evacuees followed the conservative evacuation.

\begin{tabular}{|c|c|c|c|c|}
\hline $\begin{array}{l}\text { Lower LI/Total } \\
\text { Population }\end{array}$ & Cloudshine & Groundshine & $\begin{array}{c}\text { Inhalation } \\
\text { Acute }\end{array}$ & $\begin{array}{c}\text { Resuspension } \\
\text { Acute }\end{array}$ \\
\hline $0-0.25 \mathrm{mi}$ & 0.09 & 0.10 & 0.82 & 0.0015 \\
\hline $0.25-0.33 \mathrm{mi}$ & 0.11 & 0.09 & 0.80 & 0.0017 \\
\hline $0.33-0.4 \mathrm{mi}$ & 0.12 & 0.09 & 0.79 & 0.0017 \\
\hline $0.4-0.5 \mathrm{mi}$ & 0.13 & 0.09 & 0.78 & 0.0018 \\
\hline $0.5-1.25 \mathrm{mi}$ & 0.17 & 0.09 & 0.75 & 0.0021 \\
\hline $1.25-1.33 \mathrm{mi}$ & 0.20 & 0.09 & 0.71 & 0.0026 \\
\hline $1.33-1.4 \mathrm{mi}$ & 0.20 & 0.09 & 0.71 & 0.0026 \\
\hline $1.4-1.5 \mathrm{mi}$ & 0.21 & 0.09 & 0.70 & 0.0027 \\
\hline $1.5-2 \mathrm{mi}$ & 0.22 & 0.09 & 0.69 & 0.0029 \\
\hline $\begin{array}{c}\text { Lower LI/Evacuee } \\
\text { Population }\end{array}$ & Cloudshine & Groundshine & $\begin{array}{c}\text { Inhalation } \\
\text { Acute }\end{array}$ & $\begin{array}{c}\text { Resuspension } \\
\text { Acute }\end{array}$ \\
\hline $0-0.25 \mathrm{mi}$ & 0.09 & 0.09 & 0.82 & 0.0007 \\
\hline $0.25-0.33 \mathrm{mi}$ & 0.12 & 0.09 & 0.80 & 0.0009 \\
\hline $0.33-0.4 \mathrm{mi}$ & 0.13 & 0.09 & 0.79 & 0.0010 \\
\hline $0.4-0.5 \mathrm{mi}$ & 0.13 & 0.09 & 0.78 & 0.0011 \\
\hline $0.5-1.25 \mathrm{mi}$ & 0.17 & 0.08 & 0.75 & 0.0015 \\
\hline $1.25-1.33 \mathrm{mi}$ & 0.20 & 0.08 & 0.71 & 0.0020 \\
\hline $1.33-1.4 \mathrm{mi}$ & 0.20 & 0.08 & 0.71 & 0.0021 \\
\hline $1.4-1.5 \mathrm{mi}$ & 0.21 & 0.08 & 0.70 & 0.0021 \\
\hline $1.5-2 \mathrm{mi}$ & 0.22 & 0.09 & 0.69 & 0.0024 \\
\hline $\begin{array}{c}\text { Lower LI/Non- } \\
\text { Evacuee Population }\end{array}$ & Cloudshine & Groundshine & $\begin{array}{c}\begin{array}{c}\text { Inhalation } \\
\text { Acute }\end{array} \\
\end{array}$ & $\begin{array}{c}\text { Resuspension } \\
\text { Acute }\end{array}$ \\
\hline $0-0.25 \mathrm{mi}$ & 0.07 & 0.25 & 0.67 & 0.0135 \\
\hline $0.25-0.33 \mathrm{mi}$ & 0.10 & 0.24 & 0.65 & 0.0131 \\
\hline $0.33-0.4 \mathrm{mi}$ & 0.11 & 0.24 & 0.64 & 0.0130 \\
\hline $0.4-0.5 \mathrm{mi}$ & 0.11 & 0.24 & 0.64 & 0.0129 \\
\hline
\end{tabular}


Table B.3.6 (continued)

\begin{tabular}{||c|c|c|c|c||}
\hline $\begin{array}{c}\text { Lower LI/Non- } \\
\text { Evacuee Population }\end{array}$ & Cloudshine & Groundshine & $\begin{array}{c}\text { Inhalation } \\
\text { Acute }\end{array}$ & $\begin{array}{c}\text { Resuspension } \\
\text { Acute }\end{array}$ \\
\hline $0.5-1.25 \mathrm{mi}$ & 0.15 & 0.23 & 0.61 & 0.0124 \\
\hline $1.25-1.33 \mathrm{mi}$ & 0.18 & 0.22 & 0.59 & 0.0120 \\
\hline $1.33-1.4 \mathrm{mi}$ & 0.19 & 0.22 & 0.58 & 0.0119 \\
\hline $1.4-1.5 \mathrm{mi}$ & 0.19 & 0.22 & 0.58 & 0.0119 \\
\hline $1.5-2 \mathrm{mi}$ & 0.21 & 0.21 & 0.56 & 0.0117 \\
\hline
\end{tabular}


Table B.4.1

Centerline Dose (Rem) to Red Marrow as function of distance by pathway for the source term MPB3. The third column is the dose to the average (weighted) population, the fourth is the dose to the population participating in the sheltering activity (95\%), and the last column is the dose to the population not participating in the sheltering (5\%).

\begin{tabular}{|c|c|c|c|c|}
\hline Organ/Pathway & Distance & $\begin{array}{c}\text { Dose to } \\
\text { Average } \\
\text { Population }\end{array}$ & $\begin{array}{l}\text { Dose to } \\
\text { Sheltered } \\
\text { Population }\end{array}$ & $\begin{array}{l}\text { Dose to Non- } \\
\text { Sheltered } \\
\text { Population }\end{array}$ \\
\hline Red Marrow Cloudshine & $0-0.25 \mathrm{mi}$ & 83.1 & 82.4 & 96.9 \\
\hline Red Marrow Cloudshine & $0.25-0.33 \mathrm{mi}$ & 60.5 & 60 & 70.6 \\
\hline Red Marrow Cloudshine & $0.33-0.4 \mathrm{mi}$ & 54.2 & 53.8 & 63.4 \\
\hline Red Marrow Cloudshine & $0.4-0.5 \mathrm{mi}$ & 48.6 & 48.2 & 56.8 \\
\hline Red Marrow Cloudshine & $0.5-1.25 \mathrm{mi}$ & 32.6 & 32.3 & 38.3 \\
\hline Red Marrow Cloudshine & $1.25-1.33 \mathrm{mi}$ & 23.4 & 23.2 & 27.6 \\
\hline Red Marrow Cloudshine & $1.33-1.4 \mathrm{mi}$ & 22.3 & 22.1 & 26.4 \\
\hline Red Marrow Cloudshine & $1.4-1.5 \mathrm{mi}$ & 21.3 & 21.1 & 25.1 \\
\hline Red Marrow Cloudshine & $1.5-2 \mathrm{mi}$ & 17.6 & 17.5 & 20.9 \\
\hline Red Marrow Groundshine & $0.0 .25 \mathrm{mi}$ & 215 & 210 & 309 \\
\hline Red Marrow Groundshine & $0.25-0.33 \mathrm{mi}$ & 111 & 109 & 160 \\
\hline Red Marrow Groundshine & $0.33-0.4 \mathrm{mi}$ & 90.8 & 88.7 & 131 \\
\hline Red Marrow Groundshine & $0.4-0.5 \mathrm{mi}$ & 74.7 & 72.9 & 108 \\
\hline Red Marrow Groundshine & $0.5-1.25 \mathrm{mi}$ & 36.3 & 35.5 & 52.9 \\
\hline Red Marrow Groundshine & $1.25-1.33 \mathrm{mi}$ & 20.5 & 20 & 30.1 \\
\hline Red Marrow Groundshine & $1.33-1.4 \mathrm{mi}$ & 19 & 18.6 & 28 \\
\hline Red Marrow Groundshine & $1.4-1.5 \mathrm{mi}$ & 17.6 & 17.1 & 25.9 \\
\hline Red Marrow Groundshine & $1.5-2 \mathrm{mi}$ & 13 & 12.7 & 19.2 \\
\hline Red Marrow Inhalation Acute & $0-0.25 \mathrm{mi}$ & 131 & 130 & 160 \\
\hline Red Marrow Inhalation Acute & $0.25-0.33 \mathrm{mi}$ & 68.1 & 67.3 & 83.3 \\
\hline Red Marrow Inhalation Acute & $0.33-0.4 \mathrm{mi}$ & 55.7 & 55 & 68.1 \\
\hline Red Marrow Inhalation Acute & $0.4-0.5 \mathrm{mi}$ & 45.9 & 45.3 & 56.1 \\
\hline Red Marrow Inhalation Acute & $0.5-1.25 \mathrm{mi}$ & 22.5 & 22.2 & 27.6 \\
\hline Red Marrow Inhalation Acute & $1.25-1.33 \mathrm{mi}$ & 12.7 & 12.5 & 15.5 \\
\hline Red Marrow Inhalation Acute & $1.33-1.4 \mathrm{mi}$ & 11.8 & 11.6 & 14.4 \\
\hline
\end{tabular}


Appendix B Auxiliary Dose Information

Table B.4.1 (continued)

\begin{tabular}{|l|l|c|c|c|}
\hline \multicolumn{1}{|c|}{ Organ/Pathway } & Distance & $\begin{array}{c}\text { Dose to } \\
\text { Average } \\
\text { Population }\end{array}$ & $\begin{array}{c}\text { Dose to } \\
\text { Sheltered } \\
\text { Population }\end{array}$ & $\begin{array}{c}\text { Dose to Non- } \\
\text { Sheltered } \\
\text { Population }\end{array}$ \\
\hline Red Marrow Inhalation Acute & $1.4-1.5 \mathrm{mi}$ & 10.9 & 10.7 & 13.3 \\
\hline Red Marrow Inhalation Acute & $1.5-2 \mathrm{mi}$ & 8.04 & 7.95 & 9.85 \\
\hline Red Marrow Resuspension Acute & $0-0.25 \mathrm{mi}$ & 3.04 & 3.03 & 3.23 \\
\hline Red Marrow Resuspension Acute & $0.25-0.33 \mathrm{mi}$ & 1.57 & 1.57 & 1.68 \\
\hline Red Marrow Resuspension Acute & $0.33-0.4 \mathrm{mi}$ & 1.28 & 1.28 & 1.37 \\
\hline Red Marrow Resuspension Acute & $0.4-0.5 \mathrm{mi}$ & 1.06 & 1.05 & 1.13 \\
\hline Red Marrow Resuspension Acute & $0.5-1.25 \mathrm{mi}$ & 0.514 & 0.511 & 0.556 \\
\hline Red Marrow Resuspension Acute & $1.25-1.33 \mathrm{mi}$ & 0.289 & 0.287 & 0.316 \\
\hline Red Marrow Resuspension Acute & $1.33-1.4 \mathrm{mi}$ & 0.268 & 0.267 & 0.294 \\
\hline Red Marrow Resuspension Acute & $1.4-1.5 \mathrm{mi}$ & 0.248 & 0.246 & 0.272 \\
\hline Red Marrow Resuspension Acute & $1.5-2 \mathrm{mi}$ & 0.183 & 0.182 & 0.202 \\
\hline Red Marrow Total Acute & $0-0.25 \mathrm{mi}$ & 433 & 425 & 570 \\
\hline Red Marrow Total Acute & $0.25-0.33 \mathrm{mi}$ & 241 & 238 & 316 \\
\hline Red Marrow Total Acute & $0.33-0.4 \mathrm{mi}$ & 202 & 199 & 264 \\
\hline Red Marrow Total Acute & $0.4-0.5 \mathrm{mi}$ & 170 & 167 & 222 \\
\hline Red Marrow Total Acute & $0.5-1.25 \mathrm{mi}$ & 92 & 90.5 & 119 \\
\hline Red Marrow Total Acute & $1.25-1.33 \mathrm{mi}$ & 56.8 & 56 & 73.5 \\
\hline Red Marrow Total Acute & $1.33-1.4 \mathrm{mi}$ & 53.4 & 52.6 & 69 \\
\hline Red Marrow Total Acute & $1.4-1.5 \mathrm{mi}$ & 49.9 & 49.2 & 64.5 \\
\hline Red Marrow Total Acute & $1.5-2 \mathrm{mi}$ & 38.8 & 38.3 & 50.2 \\
\hline & & & & \\
\hline
\end{tabular}


Table B.4.2

Centerline Dose (Rem) to Lungs as a function of distance by pathway for the source term MPB3. The third column is the dose to the average (weighted) population, the fourth is the exposure to the population participating in the sheltering activity ( $95 \%)$, and the last column is the dose to the population not participating in the sheltering (5\%).

\begin{tabular}{|c|c|c|c|c|}
\hline Organ/Pathway & Distance & $\begin{array}{c}\text { Dose to } \\
\text { Average } \\
\text { Population }\end{array}$ & $\begin{array}{l}\text { Dose to } \\
\text { Sheltered } \\
\text { Population }\end{array}$ & $\begin{array}{l}\text { Dose to Non- } \\
\text { Sheltered } \\
\text { Population }\end{array}$ \\
\hline Lungs Cloudshine & $0-0.25 \mathrm{mi}$ & 84 & 83.3 & 97.9 \\
\hline Lungs Cloudshine & $0.25-0.33 \mathrm{mi}$ & 61.1 & 60.6 & 71.4 \\
\hline Lungs Cloudshine & $0.33-0.4 \mathrm{mi}$ & 54.8 & 54.4 & 64.1 \\
\hline Lungs Cloudshine & $0.4-0.5 \mathrm{mi}$ & 49.1 & 48.7 & 57.5 \\
\hline Lungs Cloudshine & $0.5-1.25 \mathrm{mi}$ & 33 & 32.7 & 38.8 \\
\hline Lungs Cloudshine & $1.25-1.33 \mathrm{mi}$ & 23.7 & 23.5 & 27.9 \\
\hline Lungs Cloudshine & $1.33-1.4 \mathrm{mi}$ & 22.6 & 22.4 & 26.7 \\
\hline Lungs Cloudshine & $1.4-1.5 \mathrm{mi}$ & 21.5 & 21.3 & 25.4 \\
\hline Lungs Cloudshine & $1.5-2 \mathrm{mi}$ & 17.9 & 17.7 & 21.2 \\
\hline Lungs Groundshine & $0-0.25 \mathrm{mi}$ & 213 & 208 & 306 \\
\hline Lungs Groundshine & $0.25-0.33 \mathrm{mi}$ & 110 & 107 & 158 \\
\hline Lungs Groundshine & $0.33-0.4 \mathrm{mi}$ & 89.8 & 87.7 & 129 \\
\hline Lungs Groundshine & $0.4-0.5 \mathrm{mi}$ & 73.8 & 72.1 & 107 \\
\hline Lungs Groundshine & $0.5-1.25 \mathrm{mi}$ & 35.9 & 35.1 & 52.3 \\
\hline Lungs Groundshine & $1.25-1.33 \mathrm{mi}$ & 20.3 & 19.8 & 29.7 \\
\hline Lungs Groundshine & $1.33-1.4 \mathrm{mi}$ & 18.8 & 18.3 & 27.6 \\
\hline Lungs Groundshine & $1.4-1.5 \mathrm{mi}$ & 17.4 & 16.9 & 25.6 \\
\hline Lungs Groundshine & $1.5-2 \mathrm{mi}$ & 12.8 & 12.5 & 19 \\
\hline Lungs Inhalation Acute & $0-0.25 \mathrm{mi}$ & 658 & 650 & 805 \\
\hline Lungs Inhalation Acute & $0.25-0.33 \mathrm{mi}$ & 342 & 338 & 418 \\
\hline Lungs Inhalation Acute & $0.33-0.4 \mathrm{mi}$ & 279 & 276 & 342 \\
\hline Lungs Inhalation Acute & $0.4-0.5 \mathrm{mi}$ & 230 & 227 & 282 \\
\hline Lungs Inhalation Acute & $0.5-1.25 \mathrm{mi}$ & 113 & 111 & 138 \\
\hline Lungs Inhalation Acute & $1.25-1.33 \mathrm{mi}$ & 63.4 & 62.6 & 77.6 \\
\hline Lungs Inhalation Acute & $1.33-1.4 \mathrm{mi}$ & 58.9 & 58.2 & 72.1 \\
\hline
\end{tabular}


Table B.4.2 (continued)

\begin{tabular}{|c|c|c|c|c|}
\hline Organ/Pathway & Distance & $\begin{array}{c}\text { Dose to } \\
\text { Average } \\
\text { Population }\end{array}$ & $\begin{array}{l}\text { Dose to } \\
\text { Sheltered } \\
\text { Population }\end{array}$ & $\begin{array}{l}\text { Dose to Non- } \\
\text { Sheltered } \\
\text { Population }\end{array}$ \\
\hline Lungs Inhalation Acute & $1.4-1.5 \mathrm{mi}$ & 54.4 & 53.8 & 66.6 \\
\hline Lungs Inhalation Acute & $1.5-2 \mathrm{mi}$ & 40.2 & 39.7 & 49.2 \\
\hline Lungs Resuspension Acute & $0-0.25 \mathrm{mi}$ & 15.3 & 15.3 & 16.3 \\
\hline Lungs Resuspension Acute & $0.25-0.33 \mathrm{mi}$ & 7.91 & 7.89 & 8.44 \\
\hline Lungs Resuspension Acute & $0.33-0.4 \mathrm{mi}$ & 6.46 & 6.43 & 6.9 \\
\hline Lungs Resuspension Acute & $0.4-0.5 \mathrm{mi}$ & 5.31 & 5.29 & 5.69 \\
\hline Lungs Resuspension Acute & $0.5-1.25 \mathrm{mi}$ & 2.58 & 2.57 & 2.8 \\
\hline Lungs Resuspension Acute & $1.25-1.33 \mathrm{mi}$ & 1.45 & 1.44 & 1.59 \\
\hline Lungs Resuspension Acute & $1.33-1.4 \mathrm{mi}$ & 1.35 & 1.34 & 1.48 \\
\hline Lungs Resuspension Acute & $1.4-1.5 \mathrm{mi}$ & 1.24 & 1.24 & 1.37 \\
\hline Lungs Resuspension Acute & $1.5-2 \mathrm{mi}$ & 0.919 & 0.913 & 1.02 \\
\hline Lungs Total Acute & $0-0.25 \mathrm{mi}$ & 970 & 957 & 1220 \\
\hline Lungs Total Acute & $0.25-0.33 \mathrm{mi}$ & 521 & 514 & 656 \\
\hline Lungs Total Acute & $0.33-0.4 \mathrm{mi}$ & 430 & 424 & 542 \\
\hline Lungs Total Acute & $0.4-0.5 \mathrm{mi}$ & 358 & 353 & 451 \\
\hline Lungs Total Acute & $0.5-1.25 \mathrm{mi}$ & 184 & 182 & 232 \\
\hline Lungs Total Acute & $1.25-1.33 \mathrm{mi}$ & 109 & 107 & 137 \\
\hline Lungs Total Acute & $1.33-1.4 \mathrm{mi}$ & 102 & 100 & 128 \\
\hline Lungs Total Acute & $1.4-1.5 \mathrm{mi}$ & 94.6 & 93.3 & 119 \\
\hline Lungs Total Acute & $1.5-2 \mathrm{mi}$ & 71.9 & 70.9 & 90.4 \\
\hline
\end{tabular}


Table B.4.3

Centerline Dose (Rem) to the Lower Large Intestine as a function of distance by pathway for the source term MPB3. The third column is the dose to the average (weighted) population, the fourth is the exposure to the population participating in the sheltering activity (95\%), and the last column is the dose to the population not participating in the sheltering (5\%).

\begin{tabular}{|c|c|c|c|c|}
\hline Organ/Pathway & Distance & $\begin{array}{c}\text { Dose to } \\
\text { Average } \\
\text { Population }\end{array}$ & $\begin{array}{c}\text { Dose to } \\
\text { Sheltered } \\
\text { Population }\end{array}$ & $\begin{array}{l}\text { Dose to Non- } \\
\text { Sheltered } \\
\text { Population }\end{array}$ \\
\hline Lower LI Cloudshine & $0-0.25 \mathrm{mi}$ & 76.5 & 75.8 & 89.1 \\
\hline Lower LI Cloudshine & $0.25-0.33 \mathrm{mi}$ & 55.7 & 55.2 & 65 \\
\hline Lower LI Cloudshine & $0.33-0.4 \mathrm{mi}$ & 49.9 & 49.5 & 58.3 \\
\hline Lower LI Cloudshine & $0.4-0.5 \mathrm{mi}$ & 44.7 & 44.3 & 52.3 \\
\hline Lower LI Cloudshine & $0.5-1.25 \mathrm{mi}$ & 30 & 29.8 & 35.3 \\
\hline Lower LI Cloudshine & $1.25-1.33 \mathrm{mi}$ & 21.6 & 21.4 & 25.4 \\
\hline Lower LI Cloudshine & $1.33-1.4 \mathrm{mi}$ & 20.6 & 20.4 & 24.3 \\
\hline Lower LI Cloudshine & $1.4-1.5 \mathrm{mi}$ & 19.6 & 19.4 & 23.1 \\
\hline Lower LI Cloudshine & $1.5-2 \mathrm{mi}$ & 16.3 & 16.1 & 19.3 \\
\hline Lower LI Groundshine & $0-0.25 \mathrm{mi}$ & 193 & 189 & 277 \\
\hline Lower LI Groundshine & $0.25-0.33 \mathrm{mi}$ & 99.8 & 97.5 & 144 \\
\hline Lower LI Groundshine & $0.33-0.4 \mathrm{mi}$ & 81.4 & 79.5 & 117 \\
\hline Lower LI Groundshine & $0.4-0.5 \mathrm{mi}$ & 67 & 65.4 & 96.7 \\
\hline Lower LI Groundshine & $0.5-1.25 \mathrm{mi}$ & 32.6 & 31.8 & 47.5 \\
\hline Lower LI Groundshine & $1.25-1.33 \mathrm{mi}$ & 18.4 & 17.9 & 27 \\
\hline Lower LI Groundshine & $1.33-1.4 \mathrm{mi}$ & 17.1 & 16.6 & 25.1 \\
\hline Lower LI Groundshine & $1.4-1.5 \mathrm{mi}$ & 15.8 & 15.4 & 23.2 \\
\hline Lower LI Groundshine & $1.5-2 \mathrm{mi}$ & 11.6 & 11.4 & 17.2 \\
\hline Lower LI Inhalation Acute & $0-0.25 \mathrm{mi}$ & 715 & 706 & 875 \\
\hline Lower LI Inhalation Acute & $0.25-0.33 \mathrm{mi}$ & 371 & 367 & 454 \\
\hline Lower LI Inhalation Acute & $0.33-0.4 \mathrm{mi}$ & 303 & 300 & 371 \\
\hline Lower LI Inhalation Acute & $0.4-0.5 \mathrm{mi}$ & 250 & 247 & 306 \\
\hline Lower LI Inhalation Acute & $0.5-1.25 \mathrm{mi}$ & 123 & 121 & 150 \\
\hline Lower LI Inhalation Acute & $1.25-1.33 \mathrm{mi}$ & 68.9 & 68.1 & 84.4 \\
\hline
\end{tabular}


Appendix B Auxiliary Dose Information

Table B.4.3 (continued)

\begin{tabular}{|c|c|c|c|c|}
\hline Organ/Pathway & Distance & $\begin{array}{c}\text { Dose to } \\
\text { Average } \\
\text { Population } \\
\end{array}$ & $\begin{array}{l}\text { Dose to } \\
\text { Sheltered } \\
\text { Population }\end{array}$ & $\begin{array}{l}\text { Dose to Non- } \\
\text { Sheltered } \\
\text { Population }\end{array}$ \\
\hline Lower LI Inhalation Acute & $1.33-1.4 \mathrm{mi}$ & 64 & 63.3 & 78.4 \\
\hline Lower LI Inhalation Acute & $1.4-1.5 \mathrm{mi}$ & 59.2 & 58.5 & 72.5 \\
\hline Lower LI Inhalation Acute & $1.5-2 \mathrm{mi}$ & 43.7 & 43.2 & 53.6 \\
\hline Lower LI Resuspension Acute & $0-0.25 \mathrm{mi}$ & 16.7 & 16.6 & 17.7 \\
\hline Lower LI Resuspension Acute & $0.25-0.33 \mathrm{mi}$ & 8.61 & 8.58 & 9.18 \\
\hline Lower LI Resuspension Acute & $0.33-0.4 \mathrm{mi}$ & 7.03 & 7.01 & 7.51 \\
\hline Lower LI Resuspension Acute & $0.4-0.5 \mathrm{mi}$ & 5.78 & 5.76 & 6.19 \\
\hline Lower LI Resuspension Acute & $0.5-1.25 \mathrm{mi}$ & 2.81 & 2.8 & 3.05 \\
\hline Lower LI Resuspension Acute & $1.25-1.33 \mathrm{mi}$ & 1.58 & 1.57 & 1.73 \\
\hline Lower LI Resuspension Acute & $1.33-1.4 \mathrm{mi}$ & 1.47 & 1.46 & 1.61 \\
\hline Lower LI Resuspension Acute & $1.4-1.5 \mathrm{mi}$ & 1.36 & 1.35 & 1.49 \\
\hline Lower LI Resuspension Acute & $1.5-2 \mathrm{mi}$ & 1 & 0.996 & 1.11 \\
\hline Lower LI Total Acute & $0-0.25 \mathrm{mi}$ & 1000 & 987 & 1260 \\
\hline Lower LI Total Acute & $0.25-0.33 \mathrm{mi}$ & 535 & 528 & 672 \\
\hline Lower LI Total Acute & $0.33-0.4 \mathrm{mi}$ & 442 & 436 & 555 \\
\hline Lower LI Total Acute & $0.4-0.5 \mathrm{mi}$ & 367 & 363 & 461 \\
\hline Lower LI Total Acute & $0.5-1.25 \mathrm{mi}$ & 188 & 186 & 236 \\
\hline Lower LI Total Acute & $1.25-1.33 \mathrm{mi}$ & 110 & 109 & 138 \\
\hline Lower LI Total Acute & $1.33-1.4 \mathrm{mi}$ & 103 & 102 & 129 \\
\hline Lower LI Total Acute & $1.4-1.5 \mathrm{mi}$ & 95.9 & 94.6 & 120 \\
\hline Lower LI Total Acute & $1.5-2 \mathrm{mi}$ & 72.6 & 71.7 & 91.2 \\
\hline
\end{tabular}


Table B.4.4

Fraction of the Centerline Dose to the Red Marrow as a function of distance and by pathway for the total population, the sheltered population and the non-sheltered population. The source term was MPB3 and the protective action was sheltering.

\begin{tabular}{|c|c|c|c|c|}
\hline $\begin{array}{l}\text { Red Marrow/ } \\
\text { Total Population }\end{array}$ & Cloudshine & Groundshine & $\begin{array}{l}\text { Inhalation } \\
\text { Acute }\end{array}$ & $\begin{array}{c}\text { Resuspension } \\
\text { Acute }\end{array}$ \\
\hline $0-0.25 \mathrm{mi}$ & 0.19 & 0.50 & 0.30 & 0.007 \\
\hline $0.25-0.33 \mathrm{mi}$ & 0.25 & 0.46 & 0.28 & 0.0065 \\
\hline $0.33-0.4 \mathrm{mi}$ & 0.27 & 0.45 & 0.28 & 0.0063 \\
\hline $0.4-0.5 \mathrm{mi}$ & 0.29 & 0.44 & 0.27 & 0.0062 \\
\hline $0.5-1.25 \mathrm{mi}$ & 0.35 & 0.39 & 0.24 & 0.0056 \\
\hline $1.25-1.33 \mathrm{mi}$ & 0.41 & 0.36 & 0.22 & 0.0051 \\
\hline $1.33-1.4 \mathrm{mi}$ & 0.42 & 0.36 & 0.22 & 0.005 \\
\hline $1.4-1.5 \mathrm{mi}$ & 0.43 & 0.35 & 0.22 & 0.005 \\
\hline $1.5-2 \mathrm{mi}$ & 0.45 & 0.34 & 0.21 & 0.0047 \\
\hline $\begin{array}{c}\text { Red Marrow/ } \\
\text { Sheltered Population }\end{array}$ & Cloudshine & Groundshine & $\begin{array}{c}\text { Inhalation } \\
\text { Acute } \\
\end{array}$ & $\begin{array}{c}\text { Resuspension } \\
\text { Acute }\end{array}$ \\
\hline $0-0.25 \mathrm{mi}$ & 0.19 & 0.49 & 0.31 & 0.0071 \\
\hline $0.25-0.33 \mathrm{mi}$ & 0.25 & 0.46 & 0.28 & 0.0066 \\
\hline $0.33-0.4 \mathrm{mi}$ & 0.27 & 0.45 & 0.28 & 0.0064 \\
\hline $0.4-0.5 \mathrm{mi}$ & 0.29 & 0.44 & 0.27 & 0.0063 \\
\hline $0.5-1.25 \mathrm{mi}$ & 0.36 & 0.39 & 0.25 & 0.0056 \\
\hline $1.25-1.33 \mathrm{mi}$ & 0.41 & 0.36 & 0.22 & 0.0051 \\
\hline $1.33-1.4 \mathrm{mi}$ & 0.42 & 0.35 & 0.22 & 0.0051 \\
\hline $1.4-1.5 \mathrm{mi}$ & 0.43 & 0.35 & 0.22 & 0.005 \\
\hline $1.5-2 \mathrm{mi}$ & 0.46 & 0.33 & 0.21 & 0.0048 \\
\hline $\begin{array}{l}\text { Red Marrow/Non- } \\
\text { Sheltered Population }\end{array}$ & Cloudshine & Groundshine & $\begin{array}{c}\text { Inhalation } \\
\text { Acute }\end{array}$ & $\begin{array}{c}\text { Resuspension } \\
\text { Acute } \\
\end{array}$ \\
\hline $0-0.25 \mathrm{mi}$ & 0.17 & 0.54 & 0.28 & 0.0057 \\
\hline $0.25-0.33 \mathrm{mi}$ & 0.22 & 0.51 & 0.26 & 0.0053 \\
\hline $0.33-0.4 \mathrm{mi}$ & 0.24 & 0.50 & 0.26 & 0.0052 \\
\hline $0.4-0.5 \mathrm{mi}$ & 0.26 & 0.49 & 0.25 & 0.0051 \\
\hline
\end{tabular}


Appendix B Auxiliary Dose Information

Table B.4.4 (continued)

\begin{tabular}{||c|c|c|c|c||}
\hline $\begin{array}{c}\text { Red Marrow/Non- } \\
\text { Sheltered Population }\end{array}$ & Cloudshine & Groundshine & $\begin{array}{c}\text { Inhalation } \\
\text { Acute }\end{array}$ & $\begin{array}{c}\text { Resuspension } \\
\text { Acute }\end{array}$ \\
\hline $0.5-1.25 \mathrm{mi}$ & 0.32 & 0.44 & 0.23 & 0.0047 \\
\hline $1.25-1.33 \mathrm{mi}$ & 0.38 & 0.41 & 0.21 & 0.0043 \\
\hline $1.33-1.4 \mathrm{mi}$ & 0.38 & 0.41 & 0.21 & 0.0043 \\
\hline $1.4-1.5 \mathrm{mi}$ & 0.39 & 0.4 & 0.21 & 0.0042 \\
\hline $1.5-2 \mathrm{mi}$ & 0.42 & 0.38 & 0.20 & 0.004 \\
\hline
\end{tabular}


Table B.4.5

Fraction of the Centerline Dose to the Lungs as a function of distance and by pathway for the total population, the sheltered population and the non-sheltered population. The source term was MPB3 and the protective action was sheltering.

\begin{tabular}{|c|c|c|c|c|}
\hline $\begin{array}{c}\text { Lungs/ } \\
\text { Total Population }\end{array}$ & Cloudshine & Groundshine & $\begin{array}{c}\text { Inhalation } \\
\text { Acute }\end{array}$ & $\begin{array}{c}\text { Resuspension } \\
\text { Acute }\end{array}$ \\
\hline $0-0.25 \mathrm{mi}$ & 0.09 & 0.22 & 0.68 & 0.0158 \\
\hline $0.25-0.33 \mathrm{mi}$ & 0.12 & 0.21 & 0.66 & 0.0152 \\
\hline $0.33-0.4 \mathrm{mi}$ & 0.13 & 0.21 & 0.65 & 0.015 \\
\hline $0.4-0.5 \mathrm{mi}$ & 0.14 & 0.21 & 0.64 & 0.0148 \\
\hline $0.5-1.25 \mathrm{mi}$ & 0.18 & 0.2 & 0.61 & 0.014 \\
\hline $1.25-1.33 \mathrm{mi}$ & 0.22 & 0.19 & 0.58 & 0.0133 \\
\hline $1.33-1.4 \mathrm{mi}$ & 0.22 & 0.18 & 0.58 & 0.0132 \\
\hline $1.4-1.5 \mathrm{mi}$ & 0.23 & 0.18 & 0.58 & 0.0131 \\
\hline $1.5-2 \mathrm{mi}$ & 0.25 & 0.18 & 0.56 & 0.0128 \\
\hline $\begin{array}{c}\text { Lungs/ } \\
\text { Sheltered Population }\end{array}$ & Cloudshine & Groundshine & $\begin{array}{c}\text { Inhalation } \\
\text { Acute }\end{array}$ & $\begin{array}{c}\text { Resuspension } \\
\text { Acute }\end{array}$ \\
\hline $0-0.25 \mathrm{mi}$ & 0.09 & 0.22 & 0.68 & 0.016 \\
\hline $0.25-0.33 \mathrm{mi}$ & 0.12 & 0.21 & 0.66 & 0.0154 \\
\hline $0.33-0.4 \mathrm{mi}$ & 0.13 & 0.21 & 0.65 & 0.0152 \\
\hline $0.4-0.5 \mathrm{mi}$ & 0.14 & 0.2 & 0.64 & 0.015 \\
\hline $0.5-1.25 \mathrm{mi}$ & 0.18 & 0.19 & 0.61 & 0.0141 \\
\hline $1.25-1.33 \mathrm{mi}$ & 0.22 & 0.19 & 0.59 & 0.0135 \\
\hline $1.33-1.4 \mathrm{mi}$ & 0.22 & 0.18 & 0.58 & 0.0134 \\
\hline $1.4-1.5 \mathrm{mi}$ & 0.23 & 0.18 & 0.58 & 0.0133 \\
\hline $1.5-2 \mathrm{mi}$ & 0.25 & 0.18 & 0.56 & 0.0129 \\
\hline $\begin{array}{c}\text { Lungs/ } \\
\text { Non-Sheltered } \\
\text { Population }\end{array}$ & Cloudshine & Groundshine & $\begin{array}{l}\text { Inhalation } \\
\text { Acute }\end{array}$ & $\begin{array}{c}\text { Resuspension } \\
\text { Acute }\end{array}$ \\
\hline $0-0.25 \mathrm{mi}$ & 0.08 & 0.25 & 0.66 & 0.0134 \\
\hline $0.25-0.33 \mathrm{mi}$ & 0.11 & 0.24 & 0.64 & 0.0129 \\
\hline $0.33-0.4 \mathrm{mi}$ & 0.12 & 0.24 & 0.63 & 0.0127 \\
\hline
\end{tabular}


Table B.4.5 (continued)

\begin{tabular}{||l|c|c|c|c|}
\hline $\begin{array}{c}\text { Lungs/ } \\
\text { Non-Sheltered } \\
\text { Population }\end{array}$ & Cloudshine & Groundshine & $\begin{array}{c}\text { Inhalation } \\
\text { Acute }\end{array}$ & $\begin{array}{c}\text { Resuspension } \\
\text { Acute }\end{array}$ \\
\hline $0.4-0.5 \mathrm{mi}$ & 0.13 & 0.24 & 0.63 & 0.0126 \\
\hline $0.5-1.25 \mathrm{mi}$ & 0.17 & 0.23 & 0.59 & 0.0121 \\
\hline $1.25-1.33 \mathrm{mi}$ & 0.20 & 0.22 & 0.57 & 0.0116 \\
\hline $1.33-1.4 \mathrm{mi}$ & 0.21 & 0.22 & 0.56 & 0.0116 \\
\hline $1.4-1.5 \mathrm{mi}$ & 0.21 & 0.22 & 0.56 & 0.0115 \\
\hline $1.5-2 \mathrm{mi}$ & 0.23 & 0.21 & 0.54 & 0.0113 \\
\hline
\end{tabular}


Table B.4.6

Fraction of the Centerline Dose to the Lower Large Intestine as a function of distance and by pathway for the total population, the sheltered population and the non-sheltered population. The source term was MPB3 and the protective action was sheltering.

\begin{tabular}{|c|c|c|c|c|}
\hline $\begin{array}{c}\text { Lower LI/ } \\
\text { Total Population }\end{array}$ & Cloudshine & Groundshine & $\begin{array}{c}\text { Inhalation } \\
\text { Acute }\end{array}$ & $\begin{array}{c}\text { Resuspension } \\
\text { Acute }\end{array}$ \\
\hline $0-0.25 \mathrm{mi}$ & 0.08 & 0.19 & 0.72 & 0.0167 \\
\hline $0.25-0.33 \mathrm{mi}$ & 0.10 & 0.19 & 0.69 & 0.0161 \\
\hline $0.33-0.4 \mathrm{mi}$ & 0.11 & 0.18 & 0.69 & 0.0159 \\
\hline $0.4-0.5 \mathrm{mi}$ & 0.12 & 0.18 & 0.68 & 0.0157 \\
\hline $0.5-1.25 \mathrm{mi}$ & 0.16 & 0.17 & 0.65 & 0.0149 \\
\hline $1.25-1.33 \mathrm{mi}$ & 0.20 & 0.17 & 0.63 & 0.0144 \\
\hline $1.33-1.4 \mathrm{mi}$ & 0.20 & 0.17 & 0.62 & 0.0143 \\
\hline $1.4-1.5 \mathrm{mi}$ & 0.20 & 0.16 & 0.62 & 0.0142 \\
\hline $1.5-2 \mathrm{mi}$ & 0.22 & 0.16 & 0.60 & 0.0138 \\
\hline $\begin{array}{c}\text { Lower LI/ } \\
\text { Sheltered Population }\end{array}$ & Cloudshine & Groundshine & $\begin{array}{c}\text { Inhalation } \\
\text { Acute }\end{array}$ & $\begin{array}{c}\text { Resuspension } \\
\text { Acute }\end{array}$ \\
\hline $0-0.25 \mathrm{mi}$ & 0.08 & 0.19 & 0.72 & 0.0168 \\
\hline $0.25-0.33 \mathrm{mi}$ & 0.10 & 0.18 & 0.70 & 0.0163 \\
\hline $0.33-0.4 \mathrm{mi}$ & 0.11 & 0.18 & 0.69 & 0.0161 \\
\hline $0.4-0.5 \mathrm{mi}$ & 0.12 & 0.18 & 0.68 & 0.0159 \\
\hline $0.5-1.25 \mathrm{mi}$ & 0.16 & 0.17 & 0.65 & 0.0151 \\
\hline $1.25-1.33 \mathrm{mi}$ & 0.20 & 0.16 & 0.62 & 0.0144 \\
\hline $1.33-1.4 \mathrm{mi}$ & 0.20 & 0.16 & 0.62 & 0.0143 \\
\hline $1.4-1.5 \mathrm{mi}$ & 0.21 & 0.16 & 0.62 & 0.0143 \\
\hline $1.5-2 \mathrm{mi}$ & 0.22 & 0.16 & 0.60 & 0.0139 \\
\hline $\begin{array}{c}\text { Lower LI/Non- } \\
\text { Sheltered Population }\end{array}$ & Cloudshine & Groundshine & $\begin{array}{c}\text { Inhalation } \\
\text { Acute }\end{array}$ & $\begin{array}{c}\text { Resuspension } \\
\text { Acute }\end{array}$ \\
\hline $0-0.25 \mathrm{mi}$ & 0.07 & 0.22 & 0.69 & 0.014 \\
\hline $0.25-0.33 \mathrm{mi}$ & 0.10 & 0.21 & 0.68 & 0.0137 \\
\hline $0.33-0.4 \mathrm{mi}$ & 0.11 & 0.21 & 0.67 & 0.0135 \\
\hline
\end{tabular}


Appendix B Auxiliary Dose Information

Table B.4.6 (continued)

\begin{tabular}{|l|c|c|c|c|}
\hline $\begin{array}{c}\text { Lower LI/Non- } \\
\text { Sheltered Population }\end{array}$ & Cloudshine & Groundshine & $\begin{array}{c}\text { Inhalation } \\
\text { Acute }\end{array}$ & $\begin{array}{c}\text { Resuspension } \\
\text { Acute }\end{array}$ \\
\hline $0.4-0.5 \mathrm{mi}$ & 0.11 & 0.21 & 0.66 & 0.0134 \\
\hline $0.5-1.25 \mathrm{mi}$ & 0.15 & 0.20 & 0.64 & 0.0129 \\
\hline $1.25-1.33 \mathrm{mi}$ & 0.18 & 0.20 & 0.61 & 0.0125 \\
\hline $1.33-1.4 \mathrm{mi}$ & 0.19 & 0.19 & 0.61 & 0.0125 \\
\hline $1.4-1.5 \mathrm{mi}$ & 0.19 & 0.19 & 0.60 & 0.0124 \\
\hline $1.5-2 \mathrm{mi}$ & 0.21 & 0.19 & 0.59 & 0.0122 \\
\hline
\end{tabular}


Table B.5.1

Dose (Rem) to the three critical organs by pathway as a function of distance to produce 1 mean early fatality, releasing only I-131. The protective action assumed was sheltering.

\begin{tabular}{|c|c|c|c|}
\hline Organ/Pathway & Distance & Case 1* & Case 2* \\
\hline Red Marrow Cloudshine & $0-0.25 \mathrm{mi}$ & 16.8 & 20.4 \\
\hline Red Marrow Cloudshine & $0.25-0.33 \mathrm{mi}$ & 12.4 & 14.4 \\
\hline Red Marrow Cloudshine & $0.33-0.4 \mathrm{mi}$ & 11.1 & 12.8 \\
\hline Red Marrow Cloudshine & $0.4-0.5 \mathrm{mi}$ & 9.99 & 11.4 \\
\hline Red Marrow Cloudshine & $0.5-1.25 \mathrm{mi}$ & 6.63 & 7.43 \\
\hline Red Marrow Cloudshine & $1.25-1.33 \mathrm{mi}$ & 4.6 & 5.28 \\
\hline Red Marrow Cloudshine & $1.33-1.4 \mathrm{mi}$ & 4.38 & 5.02 \\
\hline Red Marrow Cloudshine & $1.4-1.5 \mathrm{mi}$ & 4.15 & 4.75 \\
\hline Red Marrow Cloudshine & $1.5-2 \mathrm{mi}$ & 3.43 & 3.97 \\
\hline Red Marrow Groundshine & $0-0.25 \mathrm{mi}$ & 352 & 371 \\
\hline Red Marrow Groundshine & $0.25-0.33 \mathrm{mi}$ & 181 & 183 \\
\hline Red Marrow Groundshine & $0.33-0.4 \mathrm{mi}$ & 147 & 147 \\
\hline Red Marrow Groundshine & $0.4-0.5 \mathrm{mi}$ & 121 & 120 \\
\hline Red Marrow Groundshine & $0.5-1.25 \mathrm{mi}$ & 58.7 & 58 \\
\hline Red Marrow Groundshine & $1.25-1.33 \mathrm{mi}$ & 34 & 35 \\
\hline Red Marrow Groundshine & $1.33-1.4 \mathrm{mi}$ & 31.6 & 32.4 \\
\hline Red Marrow Groundshine & $1.4-1.5 \mathrm{mi}$ & 29.1 & 29.9 \\
\hline Red Marrow Groundshine & $1.5-2 \mathrm{mi}$ & 22.2 & 23 \\
\hline Red Marrow Inhalation Acute & $0-0.25 \mathrm{mi}$ & 42.8 & 46.5 \\
\hline Red Marrow Inhalation Acute & $0.25-0.33 \mathrm{mi}$ & 22.1 & 22.9 \\
\hline Red Marrow Inhalation Acute & $0.33-0.4 \mathrm{mi}$ & 18 & 18.5 \\
\hline Red Marrow Inhalation Acute & $0.4-0.5 \mathrm{mi}$ & 14.7 & 15.1 \\
\hline Red Marrow Inhalation Acute & $0.5-1.25 \mathrm{mi}$ & 7.19 & 7.32 \\
\hline Red Marrow Inhalation Acute & $1.25-1.33 \mathrm{mi}$ & 4.16 & 4.42 \\
\hline Red Marrow Inhalation Acute & $1.33-1.4 \mathrm{mi}$ & 3.86 & 4.1 \\
\hline Red Marrow Inhalation Acute & $1.4-1.5 \mathrm{mi}$ & 3.56 & 3.79 \\
\hline Red Marrow Inhalation Acute & $1.5-2 \mathrm{mi}$ & 2.71 & 2.92 \\
\hline
\end{tabular}


Table B.5.1 (continued)

\begin{tabular}{|c|c|c|c|}
\hline Organ/Pathway & Distance & Case 1* & Case 2* \\
\hline Red Marrow Resuspension Acute & $0-0.25 \mathrm{mi}$ & 1.61 & 1.62 \\
\hline Red Marrow Resuspension Acute & $0.25-0.33 \mathrm{mi}$ & 0.829 & 0.797 \\
\hline Red Marrow Resuspension Acute & $0.33-0.4 \mathrm{mi}$ & 0.675 & 0.643 \\
\hline Red Marrow Resuspension Acute & $0.4-0.5 \mathrm{mi}$ & 0.553 & 0.523 \\
\hline Red Marrow Resuspension Acute & $0.5-1.25 \mathrm{mi}$ & 0.269 & 0.253 \\
\hline Red Marrow Resuspension Acute & $1.25-1.33 \mathrm{mi}$ & 0.156 & 0.152 \\
\hline Red Marrow Resuspension Acute & $1.33-1.4 \mathrm{mi}$ & 0.144 & 0.141 \\
\hline Red Marrow Resuspension Acute & $1.4-1.5 \mathrm{mi}$ & 0.133 & 0.13 \\
\hline Red Marrow Resuspension Acute & $1.5-2 \mathrm{mi}$ & 0.101 & 0.1 \\
\hline Red Marrow Total Acute & $0-0.25 \mathrm{mi}$ & 413 & 440 \\
\hline Red Marrow Total Acute & $0.25-0.33 \mathrm{mi}$ & 216 & 221 \\
\hline Red Marrow Total Acute & $0.33-0.4 \mathrm{mi}$ & 177 & 179 \\
\hline Red Marrow Total Acute & $0.4-0.5 \mathrm{mi}$ & 146 & 147 \\
\hline Red Marrow Total Acute & $0.5-1.25 \mathrm{mi}$ & 72.8 & 73 \\
\hline Red Marrow Total Acute & $1.25-1.33 \mathrm{mi}$ & 42.9 & 44.8 \\
\hline Red Marrow Total Acute & $1.33-1.4 \mathrm{mi}$ & 39.9 & 41.7 \\
\hline Red Marrow Total Acute & $1.4-1.5 \mathrm{mi}$ & 37 & 38.6 \\
\hline Red Marrow Total Acute & $1.5-2 \mathrm{mi}$ & 28.4 & 30 \\
\hline Lungs Cloudshine & $0-0.25 \mathrm{mi}$ & 16.4 & 19.8 \\
\hline Lungs Cloudshine & $0.25-0.33 \mathrm{mi}$ & 12.1 & 14 \\
\hline Lungs Cloudshine & $0.33-0.4 \mathrm{mi}$ & 10.8 & 12.5 \\
\hline Lungs Cloudshine & $0.4-0.5 \mathrm{mi}$ & 9.71 & 11.1 \\
\hline Lungs Cloudshine & $0.5-1.25 \mathrm{mi}$ & 6.44 & 7.22 \\
\hline Lungs Cloudshine & $1.25-1.33 \mathrm{mi}$ & 4.47 & 5.13 \\
\hline Lungs Cloudshine & $1.33-1.4 \mathrm{mi}$ & 4.26 & 4.87 \\
\hline Lungs Cloudshine & $1.4-1.5 \mathrm{mi}$ & 4.03 & 4.61 \\
\hline Lungs Cloudshine & $1.5-2 \mathrm{mi}$ & 3.33 & 3.86 \\
\hline Lungs Groundshine & $0-0.25 \mathrm{mi}$ & 342 & 362 \\
\hline Lungs Groundshine & $0.25-0.33 \mathrm{mi}$ & 176 & 178 \\
\hline
\end{tabular}


Table B.5.1 (continued)

\begin{tabular}{|c|c|c|c|}
\hline Organ/Pathway & Distance & Case 1* & Case $2^{\circ}$ \\
\hline Lungs Groundshine & $0.33-0.4 \mathrm{mi}$ & 143 & 143 \\
\hline Lungs Groundshine & $0.4-0.5 \mathrm{mi}$ & 118 & 117 \\
\hline Lungs Groundshine & $0.5-1.25 \mathrm{mi}$ & 57.2 & 56.5 \\
\hline Lungs Groundshine & $1.25-1.33 \mathrm{mi}$ & 33.1 & 34 \\
\hline Lungs Groundshine & $1.33-1.4 \mathrm{mi}$ & 30.7 & 31.6 \\
\hline Lungs Groundshine & $1.4-1.5 \mathrm{mi}$ & 28.3 & 29.1 \\
\hline Lungs Groundshine & $1.5-2 \mathrm{mi}$ & 21.6 & 22.4 \\
\hline Lungs Inhalation Acute & $0-0.25 \mathrm{mi}$ & 552 & 600 \\
\hline Lungs Inhalation Acute & $0.25-0.33 \mathrm{mi}$ & 285 & 296 \\
\hline Lungs Inhalation Acute & $0.33-0.4 \mathrm{mi}$ & 232 & 238 \\
\hline Lungs Inhalation Acute & $0.4-0.5 \mathrm{mi}$ & 190 & 194 \\
\hline Lungs Inhalation Acute & $0.5-1.25 \mathrm{mi}$ & 92.8 & 94.4 \\
\hline Lungs Inhalation Acute & $1.25-1.33 \mathrm{mi}$ & 53.7 & 57 \\
\hline Lungs Inhalation Acute & $1.33-1.4 \mathrm{mi}$ & 49.8 & 52.9 \\
\hline Lungs Inhalation Acute & $1.4-1.5 \mathrm{mi}$ & 46 & 48.9 \\
\hline Lungs Inhalation Acute & $1.5-2 \mathrm{mi}$ & 35 & 37.7 \\
\hline Lungs Kesuspension Acute & $0-0.25 \mathrm{mi}$ & 20.8 & 20.9 \\
\hline Lungs Resuspension Acute & $0.25-0.33 \mathrm{mi}$ & 10.7 & 10.3 \\
\hline Lungs Resuspension Acute & $0.33-0.4 \mathrm{mi}$ & 8.7 & 8.29 \\
\hline Lungs Resuspension Acute & $0.4-0.5 \mathrm{mi}$ & 7.14 & 6.75 \\
\hline Lungs Resuspension Acute & $0.5-1.25 \mathrm{mi}$ & 3.47 & 3.26 \\
\hline Lungs Resuspension Acute & $1.25-1.33 \mathrm{mi}$ & 2.01 & 1.96 \\
\hline Lungs Resuspension Acute & $1.33-1.4 \mathrm{mi}$ & 1.86 & 1.82 \\
\hline Lungs Resuspension Acute & $1.4-1.5 \mathrm{mi}$ & 1.72 & 1.68 \\
\hline Lungs Resuspension Acute & $1.5-2 \mathrm{mi}$ & 1.31 & 1.29 \\
\hline Lungs Total Acute & $0-0.25 \mathrm{mi}$ & 931 & 1000 \\
\hline Lungs Total Acute & $0.25-0.33 \mathrm{mi}$ & 484 & 498 \\
\hline Lungs Total Acute & $0.33-0.4 \mathrm{mi}$ & 395 & 403 \\
\hline Lungs Total Acute & $0.4-0.5 \mathrm{mi}$ & 325 & 329 \\
\hline
\end{tabular}


Appendix B Auxiliary Dose Information

Table B.5.1 (continued)

\begin{tabular}{|c|c|c|c|}
\hline Organ/Pathway & Distance & Case $1^{*}$ & Case 2* \\
\hline Lungs Total Acute & $0.5-1.25 \mathrm{mi}$ & 160 & 161 \\
\hline Lungs Total Acute & $1.25-1.33 \mathrm{mi}$ & 93.3 & 98.1 \\
\hline Lungs Total Acute & $1.33-1.4 \mathrm{mi}$ & 86.6 & 91.2 \\
\hline Lungs Total Acute & $1.4-1.5 \mathrm{mi}$ & 80 & 84.3 \\
\hline Lungs Total Acute & $1.5 .2 \mathrm{mi}$ & 61.2 & 65.3 \\
\hline Lower LI Cloudshine & $0-0.25 \mathrm{mi}$ & 14.7 & 17.8 \\
\hline Lower LI Cloudshine & $0.25-0.33 \mathrm{mi}$ & 10.8 & 12.6 \\
\hline Lower UI Cloudshine & $0.33-0.4 \mathrm{mi}$ & 9.72 & 11.2 \\
\hline Lower LI Cloudshine & $0.4-0.5 \mathrm{mi}$ & 8.73 & 9.93 \\
\hline Lower LI Cloudshine & $0.5-1.25 \mathrm{mi}$ & 5.79 & 6.49 \\
\hline Lower LI Cloudshine & $1.25-1.33 \mathrm{mi}$ & 4.02 & 4.61 \\
\hline Lower LI Cloudshine & $1.33-1.4 \mathrm{mi}$ & 3.82 & 4.38 \\
\hline Lower LI Cloudshine & $1.4-1.5 \mathrm{mi}$ & 3.62 & 4.14 \\
\hline Lower LI Cloudshine & $1.5-2 \mathrm{mi}$ & 3 & 3.47 \\
\hline Lower LI Groundshine & $0-0.25 \mathrm{mi}$ & 308 & 325 \\
\hline Lower LI Groundshine & $0.25-0.33 \mathrm{mi}$ & 158 & 160 \\
\hline Lower LI Groundshine & $0.33-0.4 \mathrm{mi}$ & 129 & 129 \\
\hline Lower LI Groundshine & $0.4-0.5 \mathrm{mi}$ & 106 & 105 \\
\hline Lower LI Groundshine & $0.5-1.25 \mathrm{mi}$ & 51.4 & 50.8 \\
\hline Lower LI Groundshine & $1.25-1.33 \mathrm{mi}$ & 29.8 & 30.6 \\
\hline Lower LI Groundshine & $1.33-1.4 \mathrm{mi}$ & 27.6 & 28.4 \\
\hline Lower LI Groundshine & $1.4-1.5 \mathrm{mi}$ & 25.5 & 26.2 \\
\hline Lower LI Groundshine & $1.5-2 \mathrm{mi}$ & 19.4 & 20.1 \\
\hline Lower LI Inhalation Acute & $0-0.25 \mathrm{mi}$ & 17.8 & 19.4 \\
\hline Lower LI Inhalation Acute & $0.25-0.33 \mathrm{mi}$ & 9.19 & 9.54 \\
\hline Lower LI Inhalation Acute & $0.33-0.4 \mathrm{mi}$ & 7.48 & 7.7 \\
\hline Lower LI Inhalation Acute & $0.4-0.5 \mathrm{mi}$ & 6.14 & 6.27 \\
\hline Lower LI Inhalation Acute & $0.5-1.25 \mathrm{mi}$ & 3 & 3.05 \\
\hline Lower LI Inhalation Acute & $1.25-1.33 \mathrm{mi}$ & 1.73 & 1.84 \\
\hline
\end{tabular}


Table B.5.1 (continued)

Appendix B Auxiliary Dose Information

\begin{tabular}{|l|l|c|c|}
\hline \multicolumn{1}{|c|}{ Organ/Pathway } & \multicolumn{1}{|c|}{ Distance } & Case $1^{*}$ & Case 2* \\
\hline Lower LI Inhalation Acute & $1.33-1.4 \mathrm{mi}$ & 1.61 & 1.71 \\
\hline Lower LI Inhalation Acute & $1.4-1.5 \mathrm{mi}$ & 1.48 & 1.58 \\
\hline Lower LI Inhalation Acute & $1.5-2 \mathrm{mi}$ & 1.13 & 1.22 \\
\hline Lower LI Resuspension Acute & $0-0.25 \mathrm{mi}$ & 0.67 & 0.675 \\
\hline Lower LI Resuspension Acute & $0.25-0.33 \mathrm{mi}$ & 0.345 & 0.332 \\
\hline Lower LI Resuspension Acute & $0.33-0.4 \mathrm{mi}$ & 0.281 & 0.268 \\
\hline Lower LI Resuspension Acute & $0.4-0.5 \mathrm{mi}$ & 0.23 & 0.218 \\
\hline Lower LI Resuspension Acute & $0.5-1.25 \mathrm{mi}$ & 0.112 & 0.105 \\
\hline Lower LI Resuspension Acute & $1.25-1.33 \mathrm{mi}$ & 0.0648 & 0.0634 \\
\hline Lower LI Resuspension Acute & $1.33-1.4 \mathrm{mi}$ & 0.0601 & 0.0588 \\
\hline Lower LI Resuspension Acute & $1.4-1.5 \mathrm{mi}$ & 0.0555 & 0.0542 \\
\hline Lower LI Resuspension Acute & $1.5-2 \mathrm{mi}$ & 0.0422 & 0.0417 \\
\hline Lower LI Total Acute & $0-0.25 \mathrm{mi}$ & 341 & 363 \\
\hline Lower LI Total Acute & $0.25-0.33 \mathrm{mi}$ & 179 & 182 \\
\hline Lower LI Total Acute & $0.33-0.4 \mathrm{mi}$ & 146 & 148 \\
\hline Lower LI Total Acute & $0.4-0.5 \mathrm{mi}$ & 121 & 121 \\
\hline Lower LI Total Acute & $0.5-1.25 \mathrm{mi}$ & 60.3 & 60.4 \\
\hline Lower LI Total Acute & $1.25-1.33 \mathrm{mi}$ & 35.6 & 37.1 \\
\hline Lower LI Total Acute & $1.33-1.4 \mathrm{mi}$ & 33.1 & 34.5 \\
\hline Lower LI Total Acute & $1.4-1.5 \mathrm{mi}$ & 30.6 & 31.9 \\
\hline Lower LI Total Acute & $1.5-2 \mathrm{mi}$ & 23.5 & 24.9 \\
\hline
\end{tabular}

*Dofined in Table 4.5.1 
Appeadix B Auxiliary Dose Information

Table B.5.2

Dose (Rem) as a function of distance to the three critical organs by pathway for the modified PWR source terms that result in one early fatality. The protective action assumed was sheltering.

\begin{tabular}{|c|c|c|c|c|}
\hline Organ/Pathway & Distance & MPWR1 & MPWR2 & MPWR3 \\
\hline Red Marrow Cloudshine & $0-0.25 \mathrm{mi}$ & 189 & 113 & 38.3 \\
\hline Red Marrow Cloudshine & $0.25-0.33 \mathrm{mi}$ & 141 & 80.7 & 28.5 \\
\hline Red Marrow Cloudshine & $0.33-0.4 \mathrm{mi}$ & 128 & 72.3 & 25.7 \\
\hline Red Marrow Cloudshine & $0.4-0.5 \mathrm{mi}$ & 115 & 64.6 & 23.2 \\
\hline Red Marrow Cloudshine & $0.5-1.25 \mathrm{mi}$ & 79 & 43.5 & 15.8 \\
\hline Red Marrow Cloudshine & $1.25-1.33 \mathrm{mi}$ & 56.4 & 31.9 & 11.2 \\
\hline Red Marrow Cloudshine & $1.33-1.4 \mathrm{mi}$ & 53.9 & 30.4 & 10.7 \\
\hline Red Marrow Cloudshine & $1.4-1.5 \mathrm{mi}$ & 51.3 & 29 & 10.1 \\
\hline Red Marrow Cloudshine & $1.5-2 \mathrm{mi}$ & 43.1 & 24.7 & 8.5 \\
\hline Red Marrow Groundshine & $0-0.25 \mathrm{mi}$ & 29.7 & 196 & 279 \\
\hline Red Marrow Groundshine & $0.25-0.33 \mathrm{mi}$ & 15.3 & 96.5 & 143 \\
\hline Red Marrow Groundshine & $0.33-0.4 \mathrm{mi}$ & 12.4 & 77.7 & 117 \\
\hline Red Marrow Groundshine & $0.4-0.5 \mathrm{mi}$ & 10.2 & 63.3 & 95.6 \\
\hline Red Marrow Groundshine & $0.5-1.25 \mathrm{mi}$ & 4.91 & 30.5 & 46.4 \\
\hline Red Marrow Groundshine & $1.25-11.33 \mathrm{mi}$ & 2.83 & 18.3 & 26.8 \\
\hline Red Marrow Groundshine & $1.33-11.4 \mathrm{mi}$ & 2.62 & 17 & 24.9 \\
\hline Red Marrow Groundshine & $1.4-1.5 \mathrm{mi}$ & 2.42 & 15.7 & 23 \\
\hline Red Marrow Groundshine & $1.5-2 \mathrm{mi}$ & 1.84 & 12 & 17.5 \\
\hline Red Marrow Inhalation Acute & $0-0.25 \mathrm{mi}$ & 8.07 & 48.1 & 78.6 \\
\hline Red Marrow Inhalation Acute & $0.25-0.33 \mathrm{mi}$ & 4.19 & 23.7 & 40.6 \\
\hline Red Marrow Inhalation Acute & $0.33-0.4 \mathrm{mi}$ & 3.42 & 19.1 & 33.1 \\
\hline Red Marrow Inhalation Acute & $0.4-0.5 \mathrm{mi}$ & 2.81 & 15.6 & 27.1 \\
\hline Red Marrow Inhalation Acute & $0.5-1.25 \mathrm{mi}$ & 1.39 & 7.58 & 13.2 \\
\hline Red Marrow Inhalation Acute & $1.25-1.33 \mathrm{mi}$ & 0.811 & 4.58 & 7.66 \\
\hline Red Marrow Inhalation Acute & $1.33-1.4 \mathrm{mi}$ & 0.754 & 4.25 & 7.11 \\
\hline Red Marrow Inhalation Acute & $1.4-1.5 \mathrm{mi}$ & 0.697 & 3.93 & 6.56 \\
\hline Red Marrow Inhalation Acute & $1.5-2 \mathrm{mi}$ & 0.534 & 3.03 & 5 \\
\hline
\end{tabular}


Table B.5.2 (continued)

Appendix B Auxiliary Dose Information

\begin{tabular}{|c|c|c|c|c|}
\hline Organ/Pathway & Distance & MPWR1 & MPWR2 & MPWR3 \\
\hline Red Marrow Resuspension Acute & $0-0.25 \mathrm{mi}$ & 0.234 & 1.63 & 2.92 \\
\hline Red Marrow Resuspension Acute & $0.25-0.33 \mathrm{mi}$ & 0.12 & 0.801 & 1.5 \\
\hline Red Marrow Resuspension Acute & $0.33-0.4 \mathrm{mi}$ & 0.098 & 0.646 & 1.22 \\
\hline Red Marrow Resuspension Acute & $0.4-0.5 \mathrm{mi}$ & 0.0804 & 0.526 & 1 \\
\hline Red Marrow Resuspension Acute & $0.5-1.25 \mathrm{mi}$ & 0.039 & 0.254 & 0.487 \\
\hline Red Marrow Resuspension Acute & $1.25-1.33 \mathrm{mi}$ & 0.0226 & 0.153 & 0.282 \\
\hline Red Marrow Resuspension Acute & $1.33-1.4 \mathrm{mi}$ & 0.0209 & 0.142 & 0.262 \\
\hline Red Marrow Resuspension Acute & $1.4-1.5 \mathrm{mi}$ & 0.0193 & 0.131 & 0.241 \\
\hline Red Marrow Resuspension Acute & $1.5-2 \mathrm{mi}$ & 0.0147 & 0.1 & 0.184 \\
\hline Red Marrow Total Acute & $0-0.25 \mathrm{mi}$ & 227 & 359 & 398 \\
\hline Red Marrow Total Acute & $0.25-0.33 \mathrm{mi}$ & 161 & 202 & 214 \\
\hline Red Marrow Total Acute & $0.33-0.4 \mathrm{mi}$ & 144 & 170 & 177 \\
\hline Red Marrow Total Acute & $0.4-0.5 \mathrm{mi}$ & 128 & 144 & 147 \\
\hline Red Marrow Total Acute & $0.5-1.25 \mathrm{mi}$ & 85.3 & 81.8 & 75.9 \\
\hline Red Marrow Total Acute & $1.25-1.33 \mathrm{mi}$ & 60.1 & 55 & 46 \\
\hline Red Marrow Total Acute & $1.33-1.4 \mathrm{mi}$ & 57.3 & 51.8 & 42.9 \\
\hline Red Marrow Total Acute & $1.4-1.5 \mathrm{mi}$ & 54.5 & 48.7 & 39.9 \\
\hline Red Marrow Total Acute & $1.5-2 \mathrm{mi}$ & 45.5 & 39.8 & 31.1 \\
\hline Lungs Cloudshine & $0-0.25 \mathrm{mi}$ & 188 & 113 & 40 \\
\hline Lungs Cloudshine & $0.25-0.33 \mathrm{mi}$ & 141 & 81.2 & 29.8 \\
\hline Lungs Cloudshine & $0.33-0.4 \mathrm{mi}$ & 127 & 72.8 & 26.9 \\
\hline Lungs Cloudshine & $0.4-0.5 \mathrm{mi}$ & 115 & 65 & 24.2 \\
\hline Lungs Cloudshine & $0.5-1.25 \mathrm{mi}$ & 78.9 & 43.8 & 16.5 \\
\hline Lungs Cloudshine & $1.25-1.33 \mathrm{mi}$ & 56.4 & 32.2 & 11.7 \\
\hline Lungs Cloudshine & $1.33-1.4 \mathrm{mi}$ & 53.9 & 30.7 & 11.2 \\
\hline Lungs Cloudshine & 1.4-1.5 mi & 51.3 & 29.2 & 10.6 \\
\hline Lungs Cloudshine & $1.5-2 \mathrm{mi}$ & 43.1 & 24.9 & 8.93 \\
\hline Lungs Groundshine & $0-0.25 \mathrm{mi}$ & 29.3 & 194 & 275 \\
\hline Lungs Groundshine & $0.25-0.33 \mathrm{mi}$ & 5.1 & 95.2 & 142 \\
\hline
\end{tabular}


Appendix B Auxiliary Dose Information

Table B.5.2 (continued)

\begin{tabular}{|c|c|c|c|c|}
\hline Organ/Pathway & Distance & MPWR1 & MPWR2 & MPWR3 \\
\hline Lungs Groundshine & $0.33-0.4 \mathrm{mi}$ & 2.2 & 76.7 & 115 \\
\hline Lungs Groundshine & $0.4-0.5 \mathrm{mi}$ & 10 & 62.5 & 94.4 \\
\hline Lungs Groundshine & $0.5-1.25 \mathrm{mi}$ & 4.85 & 30.1 & 45.8 \\
\hline Lungs Groundshine & $1.25-1.33 \mathrm{mi}$ & 2.8 & 18.1 & 26.5 \\
\hline Lungs Groundshine & $1.33-1.4 \mathrm{mi}$ & 2.59 & 16.8 & 24.6 \\
\hline Lungs Groundshine & $1.4-1.5 \mathrm{mi}$ & 2.39 & 15.5 & 22.7 \\
\hline Lungs Groundshine & $1.5-2 \mathrm{mi}$ & 1.81 & 11.9 & 17.2 \\
\hline Lungs Inhalation Acute & $0-0.25 \mathrm{mi}$ & 53.3 & 311 & 462 \\
\hline Lungs Inhalation Acute & $0.25-0.33 \mathrm{mi}$ & 27.6 & 153 & 238 \\
\hline Lungs Inhalation Acute & $0.33-0.4 \mathrm{mi}$ & 22.5 & 123 & 194 \\
\hline Lungs Inhalation Acute & $0.4-0.5 \mathrm{mi}$ & 18.5 & 100 & 159 \\
\hline Lungs Inhalation Acute & $0.5-1.25 \mathrm{mi}$ & 9.06 & 48.8 & 77.6 \\
\hline Lungs Inhalation Acute & $1.25-1.33 \mathrm{mi}$ & 5.27 & 29.4 & 44.9 \\
\hline Lungs Inhalation Acute & $1.33-1.4 \mathrm{mi}$ & 4.89 & 27.3 & 41.6 \\
\hline Lungs Inhalation Acute & 1.4-1.5 mi & 4.52 & 25.2 & 38.4 \\
\hline Lungs Inhalation Acute & $1.5-2 \mathrm{mi}$ & 3.45 & 19.4 & 29.2 \\
\hline Lungs Resuspension Acute & $0-0.25 \mathrm{mi}$ & 1.62 & 10.6 & 17.3 \\
\hline Lungs Resuspension Acute & $0.25-0.33 \mathrm{mi}$ & 0.833 & 5.22 & 8.9 \\
\hline Lungs Resuspension Acute & $0.33-0.4 \mathrm{mi}$ & 0.677 & 4.21 & 7.24 \\
\hline Lungs Resuspension Acute & $0.4-0.5 \mathrm{mi}$ & 0.555 & 3.42 & 5.93 \\
\hline Lungs Resuspension Acute & $0.5-1.25 \mathrm{mi}$ & 0.269 & 1.65 & 2.88 \\
\hline Lungs Resuspension Acute & $1.25-1.33 \mathrm{mi}$ & 0.155 & 0.991 & 1.67 \\
\hline Lungs Resuspension Acute & $1.33-1.4 \mathrm{mi}$ & 0.144 & 0.919 & 1.55 \\
\hline Lungs Resuspension Acute & 1.4-1.5 mi & 0.133 & 0.847 & 1.43 \\
\hline Lungs Resuspension Acute & $1.5-2 \mathrm{mi}$ & 0.101 & 0.651 & 1.08 \\
\hline Lungs Total Acute & $0-0.25 \mathrm{mi}$ & 273 & 628 & 794 \\
\hline Lungs Total Acute & $0.25-0.33 \mathrm{mi}$ & 184 & 334 & 419 \\
\hline Lungs Total Acute & $0.33-0.4 \mathrm{mi}$ & 163 & 277 & 343 \\
\hline Lungs Total Acute & $0.4-0.5 \mathrm{mi}$ & 144 & 231 & 284 \\
\hline
\end{tabular}


Table B.5.2 (continued)

Appendix B Auxiliary Dose Information

\begin{tabular}{|c|c|c|c|c|}
\hline Organ/Pathway & Distance & MPWR1 & MPWR2 & MPWR3 \\
\hline Lungs Total Acute & $0.5-1.25 \mathrm{mi}$ & 93.1 & 124 & 143 \\
\hline Lungs Total Acute & $1.25-1.33 \mathrm{mi}$ & 64.6 & 80.6 & 84.7 \\
\hline Lungs Total Acute & $1.33-1.4 \mathrm{mi}$ & 61.5 & 75.7 & 78.9 \\
\hline Lungs Total Acute & $1.4-1.5 \mathrm{mi}$ & 58.4 & 70.7 & 73.1 \\
\hline Lungs Total Acute & $1.5-2 \mathrm{mi}$ & 48.5 & 56.8 & 56.5 \\
\hline Lower LI Cloudshine & $0-0.25 \mathrm{mi}$ & 174 & 104 & 35.7 \\
\hline Lower LI Cloudshine & $0.25-0.33 \mathrm{mi}$ & 130 & 74.3 & 26.6 \\
\hline Lower LI Cloudshine & $0.33-0.4 \mathrm{mi}$ & 117 & 66.6 & 24 \\
\hline Lower LI Cloudshine & $0.4-0.5 \mathrm{mi}$ & 106 & 59.5 & 21.7 \\
\hline Lower LI Cloudshine & $0.5-1.25 \mathrm{mi}$ & 72.7 & 40.1 & 14.7 \\
\hline Lower LI Cloudshine & $1.25-1.33 \mathrm{mi}$ & 51.9 & 29.4 & 10.4 \\
\hline Lower LI Cloudshine & $1.33-1.4 \mathrm{mi}$ & 49.6 & 28.1 & 9.98 \\
\hline Lower LI Cloudshine & $1.4-1.5 \mathrm{mi}$ & 47.2 & 26.7 & 9.49 \\
\hline Lower LI Cloudshine & $1.5-2 \mathrm{mi}$ & 39.7 & 22.8 & 7.96 \\
\hline Lower LI Groundshine & $0-0.25 \mathrm{mi}$ & 26.5 & 176 & 249 \\
\hline Lower LI Groundshine & $0.25-0.33 \mathrm{mi}$ & 13.6 & 86.2 & 128 \\
\hline Lower LI Groundshine & $0.33-0.4 \mathrm{mi}$ & 11.1 & 69.5 & 104 \\
\hline Lower LI Groundshine & $0.4-0.5 \mathrm{mi}$ & 9.07 & 56.5 & 85.4 \\
\hline Lower LI Groundshine & $0.5-1.25 \mathrm{mi}$ & 4.39 & 27.3 & 41.4 \\
\hline Lower LI Groundshine & $1.25-1.33 \mathrm{mi}$ & 2.53 & 16.4 & 24 \\
\hline Lower LI Groundshine & $1.33-1.4 \mathrm{mi}$ & 2.35 & 15.2 & 22.2 \\
\hline Lower LI Groundshine & $1.4-1.5 \mathrm{mi}$ & 2.16 & 14 & 20.5 \\
\hline Lower LI Groundshine & $1.5-2 \mathrm{mi}$ & 1.64 & 10.7 & 15.6 \\
\hline Lower LI Inhalation Acute & $0-0.25 \mathrm{mi}$ & 33.3 & 241 & 402 \\
\hline Lower LI Inhalation Acute & $0.25-0.33 \mathrm{mi}$ & 17.2 & 119 & 208 \\
\hline Lower LI Inhalation Acute & $0.33-0.4 \mathrm{mi}$ & 14 & 95.6 & 169 \\
\hline Lower LI Inhalation Acute & $0.4-0.5 \mathrm{mi}$ & 11.5 & 77.9 & 139 \\
\hline Lower LI Inhalation Acute & $0.5-1.25 \mathrm{mi}$ & 5.63 & 37.9 & 67.7 \\
\hline Lower LI Inhalation Acute & $1.25-1.33 \mathrm{mi}$ & 3.26 & 22.9 & 39.2 \\
\hline
\end{tabular}


Appendix B Auxiliary Dose Information

Table B.5.2 (continued)

\begin{tabular}{||l|l|c|c|c||}
\hline \multicolumn{1}{|c|}{ Organ/Pathway } & \multicolumn{1}{|c|}{ Distance } & MPWR1 & MPWR2 & MPWR3 \\
\hline Lower LI Inhalation Acute & $1.33-1.4 \mathrm{mi}$ & 3.03 & 21.2 & 36.3 \\
\hline Lower LI Inhalation Acute & $1.4-1.5 \mathrm{mi}$ & 2.8 & 19.6 & 33.5 \\
\hline Lower LI Inhalation Acute & $1.5-2 \mathrm{mi}$ & 2.13 & 15.1 & 25.5 \\
\hline Lower LI Resuspension Acute & $0-0.25 \mathrm{mi}$ & 1.19 & 8.35 & 15.1 \\
\hline Lower LI Resuspension Acute & $0.25-0.33 \mathrm{mi}$ & 0.612 & 4.1 & 7.78 \\
\hline Lower LI Resuspension Acute & $0.33-0.4 \mathrm{mi}$ & 0.498 & 3.31 & 6.33 \\
\hline Lower LI Resuspension Acute & $0.4-0.5 \mathrm{mi}$ & 0.408 & 2.69 & 5.19 \\
\hline Lower LI Resuspension Acute & $0.5-1.25 \mathrm{mi}$ & 0.198 & 1.3 & 2.52 \\
\hline Lower LI Resuspension Acute & $1.25-1.33 \mathrm{mi}$ & 0.115 & 0.783 & 1.46 \\
\hline Lower LI Resuspension Acute & $1.33-1.4 \mathrm{mi}$ & 0.106 & 0.726 & 1.36 \\
\hline Lower LI Resuspension Acute & $1.4-1.5 \mathrm{mi}$ & 0.0982 & 0.669 & 1.25 \\
\hline Lower LI Resuspension Acute & $1.5-2 \mathrm{mi}$ & 0.0747 & 0.514 & 0.951 \\
\hline Lower LI Total Acute & $0-0.25 \mathrm{mi}$ & 235 & 528 & 702 \\
\hline Lower LI Total Acute & $0.25-0.33 \mathrm{mi}$ & 161 & 283 & 370 \\
\hline Lower LI Total Acute & $0.33-0.4 \mathrm{mi}$ & 143 & 235 & 304 \\
\hline Lower LI Total Acute & $0.4-0.5 \mathrm{mi}$ & 127 & 197 & 251 \\
\hline Lower LI Total Acute & $0.5-1.25 \mathrm{mi}$ & 82.9 & 106 & 126 \\
\hline Lower LI Total Acute & $1.25-1.33 \mathrm{mi}$ & 57.8 & 69.4 & 75 \\
\hline Lower LI Total Acute & $1.33-1.4 \mathrm{mi}$ & 55.1 & 65.2 & 69.9 \\
\hline Lower LI Total Acute & $1.4-1.5 \mathrm{mi}$ & 52.3 & 61 & 64.8 \\
\hline Lower LI Total Acute & $1.5-2 \mathrm{mi}$ & 43.6 & 49.1 & 50 \\
\hline
\end{tabular}


Table B.5.3

Fraction of dose as a function of distance to the three critical organs by pathway, releasing only $1-131$. The protective action assumed was sheltering. Cases 1 and 2 are defined in Table 4.5.1.

\begin{tabular}{|c|c|c|c|c|}
\hline & Cloudshine & Groundshine & $\begin{array}{l}\text { Inhalation } \\
\text { Acute }\end{array}$ & $\begin{array}{c}\text { Resuspension } \\
\text { Acute }\end{array}$ \\
\hline \multicolumn{5}{|c|}{ Red Marrow, Case 1} \\
\hline $0-0.25 \mathrm{mi}$ & 0.041 & 0.852 & 0.104 & 0.0039 \\
\hline $0.25-0.33 \mathrm{mi}$ & 0.057 & 0.838 & 0.102 & 0.0038 \\
\hline $0.33-0.4 \mathrm{mi}$ & 0.063 & 0.831 & 0.102 & 0.0038 \\
\hline $0.4-0.5 \mathrm{mi}$ & 0.068 & 0.829 & 0.101 & 0.0038 \\
\hline $0.5-1.25 \mathrm{mi}$ & 0.091 & 0.806 & 0.099 & 0.0037 \\
\hline $1.25-1.33 \mathrm{mi}$ & 0.107 & 0.793 & 0.097 & 0.0036 \\
\hline $1.33-1.4 \mathrm{mi}$ & 0.11 & 0.792 & 0.097 & 0.0036 \\
\hline $1.4-1.5 \mathrm{mi}$ & 0.112 & 0.786 & 0.096 & 0.0036 \\
\hline $1.5-2 \mathrm{mi}$ & 0.121 & 0.782 & 0.095 & 0.0036 \\
\hline \multicolumn{5}{|c|}{ Red Marrow, Case 2} \\
\hline $0.0 .25 \mathrm{mi}$ & 0.046 & 0.843 & 0.106 & 0.0037 \\
\hline $0.25-0.33 \mathrm{mi}$ & 0.065 & 0.828 & 0.104 & 0.0036 \\
\hline $0.33-0.4 \mathrm{mi}$ & 0.072 & 0.821 & 0.103 & 0.0036 \\
\hline $0.4-0.5 \mathrm{mi}$ & 0.078 & 0.816 & 0.103 & 0.0036 \\
\hline $0.5-1.25 \mathrm{mi}$ & 0.102 & 0.795 & 0.10 & 0.0035 \\
\hline $1.25-1.33 \mathrm{mi}$ & 0.118 & 0.781 & 0.099 & 0.0034 \\
\hline $1.33-1.4 \mathrm{mi}$ & 0.12 & 0.777 & 0.098 & 0.0034 \\
\hline $1.4-1.5 \mathrm{mi}$ & 0.123 & 0.775 & 0.098 & 0.0034 \\
\hline $1.5-2 \mathrm{mi}$ & 0.132 & 0.767 & 0.097 & 0.0033 \\
\hline \multicolumn{5}{|c|}{ Lungs, Case 1} \\
\hline $0-0.25 \mathrm{mi}$ & 0.018 & 0.367 & 0.593 & 0.022 \\
\hline $0.25-0.33 \mathrm{mi}$ & 0.025 & 0.364 & 0.589 & 0.022 \\
\hline $0.33-0.4 \mathrm{mi}$ & 0.027 & 0.362 & 0.587 & 0.022 \\
\hline $0.4-0.5 \mathrm{mi}$ & 0.03 & 0.363 & 0.585 & 0.022 \\
\hline $0.5-1.25 \mathrm{mi}$ & 0.04 & 0.358 & 0.58 & 0.022 \\
\hline
\end{tabular}


Table B.5.3 (continued)

\begin{tabular}{|c|c|c|c|c|}
\hline & Cloudshine & Groundshine & $\begin{array}{c}\text { Inhalation } \\
\text { Acute }\end{array}$ & $\begin{array}{c}\text { Resuspension } \\
\text { Acute }\end{array}$ \\
\hline $1.25-1.33 \mathrm{mi}$ & 0.048 & 0.355 & 0.576 & 0.022 \\
\hline $1.33-1.4 \mathrm{mi}$ & 0.049 & 0.355 & 0.575 & 0.021 \\
\hline $1.4-1.5 \mathrm{mi}$ & 0.05 & 0.354 & 0.575 & 0.022 \\
\hline $1.5-2 \mathrm{mi}$ & 0.054 & 0.353 & 0.572 & 0.021 \\
\hline \multicolumn{5}{|l|}{ Lungs, Case 2} \\
\hline $0-0.25 \mathrm{mi}$ & 0.02 & 0.362 & 0.50 & 0.021 \\
\hline $0.25-0.33 \mathrm{mi}$ & 0.028 & 0.357 & 0.594 & 0.021 \\
\hline $0.33-0.4 \mathrm{mi}$ & 0.031 & 0.355 & 0.591 & 0.021 \\
\hline $0.4-0.5 \mathrm{mi}$ & 0.034 & 0.356 & 0.59 & 0.021 \\
\hline $0.5-1.25 \mathrm{mi}$ & 0.045 & 0.351 & 0.586 & 0.02 \\
\hline $1.25-1.33 \mathrm{mi}$ & 0.052 & 0.347 & 0.581 & 0.02 \\
\hline $1.33-1.4 \mathrm{mi}$ & 0.053 & 0.346 & 0.58 & 0.02 \\
\hline $1.4-1.5 \mathrm{mi}$ & 0.055 & 0.345 & 0.58 & 0.02 \\
\hline $1.5-2 \mathrm{mi}$ & 0.059 & 0.343 & 0.577 & 0.02 \\
\hline \multicolumn{5}{|c|}{ Lower LI, Case 1} \\
\hline $0-0.25 \mathrm{mi}$ & 0.043 & 0.903 & 0.052 & 0.002 \\
\hline $0.25-0.33 \mathrm{mi}$ & 0.06 & 0.883 & 0.051 & 0.0019 \\
\hline $0.33-0.4 \mathrm{mi}$ & 0.067 & 0.884 & 0.051 & 0.0019 \\
\hline $0.4-0.5 \mathrm{mi}$ & 0.072 & 0.876 & 0.051 & 0.0019 \\
\hline $0.5-1.25 \mathrm{mi}$ & 0.096 & 0.852 & 0.05 & 0.0019 \\
\hline $1.25-1.33 \mathrm{mi}$ & 0.113 & 0.837 & 0.049 & 0.0018 \\
\hline $1.33-1.4 \mathrm{mi}$ & 0.115 & 0.834 & 0.049 & 0.0018 \\
\hline $1.4-1.5 \mathrm{mi}$ & 0.118 & 0.833 & 0.048 & 0.0018 \\
\hline $1.5-2 \mathrm{mi}$ & 0.128 & 0.826 & 0.048 & 0.0018 \\
\hline \multicolumn{5}{|l|}{ Lower LI, C2 } \\
\hline $0-0.25 \mathrm{mi}$ & 0.049 & 0.895 & 0.053 & 0.0019 \\
\hline $0.25-0.33 \mathrm{mi}$ & 0.069 & 0.879 & 0.052 & 0.0018 \\
\hline $0.33-0.4 \mathrm{mi}$ & 0.076 & 0.872 & 0.052 & 0.0018 \\
\hline $0.4-0.5 \mathrm{mi}$ & 0.082 & 0.868 & 0.052 & 0.0018 \\
\hline
\end{tabular}


Table B.5.3 (continued)

Appendix B Auxiliary Dose Information

\begin{tabular}{||l|c|c|c|c||}
\hline & Cloudshine & Groundshine & $\begin{array}{c}\text { Inhalation } \\
\text { Acute }\end{array}$ & $\begin{array}{c}\text { Resuspension } \\
\text { Acute }\end{array}$ \\
\hline $0.5-1.25 \mathrm{mi}$ & 0.107 & 0.841 & 0.05 & 0.0017 \\
\hline $1.25-1.33 \mathrm{mi}$ & 0.124 & 0.825 & 0.05 & 0.0017 \\
\hline $1.33-1.4 \mathrm{mi}$ & 0.127 & 0.823 & 0.05 & 0.0017 \\
\hline $1.4-1.5 \mathrm{mi}$ & 0.13 & 0.821 & 0.05 & 0.0017 \\
\hline $1.5-2 \mathrm{mi}$ & 0.139 & 0.807 & 0.049 & 0.0017 \\
\hline
\end{tabular}


Appendix B Auxiliary Dose Information

Table B.5.4

Fraction of dose as a function of distance to the three critical organs by pathway for the modified PWR source terms. The protective action assumed was sheltering.

\begin{tabular}{|c|c|c|c|c|}
\hline & Cloudshine & Groundshine & $\begin{array}{c}\text { Inhalation } \\
\text { Acute }\end{array}$ & $\begin{array}{c}\text { Resuspension } \\
\text { Acute }\end{array}$ \\
\hline \multicolumn{5}{|c|}{ Red Marrow, MPWR1 } \\
\hline $0.0 .25 \mathrm{mi}$ & 0.833 & 0.131 & 0.036 & 0.001 \\
\hline $0.25-0.33 \mathrm{mi}$ & 0.876 & 0.095 & 0.026 & 0.00075 \\
\hline $0.33-0.4 \mathrm{mi}$ & 0.889 & 0.086 & 0.024 & 0.00068 \\
\hline $0.4-0.5 \mathrm{mi}$ & 0.898 & 0.08 & 0.022 & 0.00063 \\
\hline $0.5-1.25 \mathrm{mi}$ & 0.926 & 0.058 & 0.016 & 0.00046 \\
\hline $1.25-1.33 \mathrm{mi}$ & 0.938 & 0.047 & 0.013 & 0.00038 \\
\hline $1.33-1.4 \mathrm{mi}$ & 0.941 & 0.046 & 0.013 & 0.00036 \\
\hline $1.4-1.5 \mathrm{mi}$ & 0.941 & 0.044 & 0.013 & 0.00035 \\
\hline $1.5-2 \mathrm{mi}$ & 0.947 & 0.04 & 0.012 & 0.00032 \\
\hline \multicolumn{5}{|c|}{ Red Marrow, MPWR2 } \\
\hline $0-0.25 \mathrm{mi}$ & 0.315 & 0.546 & 0.134 & 0.0045 \\
\hline $0.25-0.33 \mathrm{mi}$ & 0.40 & 0.478 & 0.117 & 0.004 \\
\hline $0.33-0.4 \mathrm{mi}$ & 0.425 & 0.457 & 0.112 & 0.0038 \\
\hline $0.4-0.5 \mathrm{mi}$ & 0.449 & 0.44 & 0.108 & 0.0037 \\
\hline $0.5-1.25 \mathrm{mi}$ & 0.532 & 0.373 & 0.093 & 0.0031 \\
\hline $1.25-1.33 \mathrm{mi}$ & 0.58 & 0.333 & 0.083 & 0.0028 \\
\hline $1.33-1.4 \mathrm{mi}$ & 0.587 & 0.328 & 0.082 & 0.0027 \\
\hline $1.4-1.5 \mathrm{mi}$ & 0.595 & 0.322 & 0.081 & 0.0027 \\
\hline $1.5-2 \mathrm{mi}$ & 0.621 & 0.302 & 0.076 & 0.0025 \\
\hline \multicolumn{5}{|c|}{ Red Marrow, MPWR3 } \\
\hline $0-0.25 \mathrm{mi}$ & 0.096 & 0.701 & 0.197 & 0.0073 \\
\hline $0.25-0.33 \mathrm{mi}$ & 0.133 & 0.668 & 0.19 & 0.007 \\
\hline $0.33-0.4 \mathrm{mi}$ & 0.145 & 0.661 & 0.187 & 0.0069 \\
\hline $0.4-0.5 \mathrm{mi}$ & 0.158 & 0.65 & 0.184 & 0.0068 \\
\hline $0.5-1.25 \mathrm{mi}$ & 0.208 & 0.611 & 0.174 & 0.0064 \\
\hline
\end{tabular}


Table B.5.4 (continued)

Appendix B Auxiliary Dose Information

\begin{tabular}{|c|c|c|c|c|}
\hline & Cloudshine & Groundshine & $\begin{array}{c}\text { Inhalation } \\
\text { Acute }\end{array}$ & $\begin{array}{c}\text { Resuspension } \\
\text { Acute }\end{array}$ \\
\hline $1.25-1.33 \mathrm{mi}$ & 0.243 & 0.583 & 0.167 & 0.0061 \\
\hline $1.33-1.4 \mathrm{mi}$ & 0.249 & 0.58 & 0.166 & 0.0061 \\
\hline $1.4-1.5 \mathrm{mi}$ & 0.253 & 0.576 & 0.164 & 0.006 \\
\hline $1.5-2 \mathrm{mi}$ & 0.273 & 0.563 & 0.161 & 0.0059 \\
\hline \multicolumn{5}{|c|}{ Lungs, MPWR1 } \\
\hline $0-0.25 \mathrm{mi}$ & 0.689 & 0.107 & 0.195 & 0.0059 \\
\hline $0.25-0.33 \mathrm{mi}$ & 0.766 & 0.082 & 0.15 & 0.0045 \\
\hline $0.33-0.4 \mathrm{mi}$ & 0.779 & 0.075 & 0.138 & 0.0042 \\
\hline $0.4-0.5 \mathrm{mi}$ & 0.799 & 0.069 & 0.128 & 0.0039 \\
\hline $0.5-1.25 \mathrm{mi}$ & 0.847 & 0.052 & 0.097 & 0.0029 \\
\hline $1.25-1.33 \mathrm{mi}$ & 0.873 & 0.043 & 0.082 & 0.0024 \\
\hline $1.33-1.4 \mathrm{mi}$ & 0.876 & 0.042 & 0.08 & 0.0023 \\
\hline $1.4-1.5 \mathrm{mi}$ & 0.878 & 0.041 & 0.077 & 0.0023 \\
\hline $1.5-2 \mathrm{mi}$ & 0.889 & 0.037 & 0.071 & 0.0021 \\
\hline \multicolumn{5}{|c|}{ Lungs, MPWR2 } \\
\hline $0-0.25 \mathrm{mi}$ & 0.18 & 0.309 & 0.495 & 0.017 \\
\hline $0.25-0.33 \mathrm{mi}$ & 0.243 & 0.285 & 0.458 & 0.016 \\
\hline $0.33-0.4 \mathrm{mi}$ & 0.263 & 0.277 & 0.444 & 0.015 \\
\hline $0.4-0.5 \mathrm{mi}$ & 0.281 & 0.271 & 0.433 & 0.015 \\
\hline $0.5-1.25 \mathrm{mi}$ & 0.353 & 0.243 & 0.394 & 0.013 \\
\hline $1.25-1.33 \mathrm{mi}$ & 0.4 & 0.225 & 0.365 & 0.012 \\
\hline $1.33-1.4 \mathrm{mi}$ & 0.406 & 0.222 & 0.361 & 0.012 \\
\hline $1.4-1.5 \mathrm{mi}$ & 0.413 & 0.219 & 0.356 & 0.012 \\
\hline $1.5-2 \mathrm{mi}$ & 0.438 & 0.21 & 0.342 & 0.011 \\
\hline \multicolumn{5}{|c|}{ Lungs, MPWR3 } \\
\hline $0-0.25 \mathrm{mi}$ & 0.05 & 0.346 & 0.582 & 0.022 \\
\hline $0.25-0.33 \mathrm{mi}$ & 0.071 & 0.339 & 0.568 & 0.021 \\
\hline $0.33-0.4 \mathrm{mi}$ & 0.078 & 0.335 & 0.566 & 0.021 \\
\hline $0.4-0.5 \mathrm{mi}$ & 0.085 & 0.332 & 0.56 & 0.021 \\
\hline
\end{tabular}


Table B.5.4 (continued)

\begin{tabular}{|c|c|c|c|c|}
\hline & Cloudshine & Groundshine & $\begin{array}{c}\text { Inhalation } \\
\text { Acute }\end{array}$ & $\begin{array}{c}\text { Resuspension } \\
\text { Acute }\end{array}$ \\
\hline $0.5-1.25 \mathrm{mi}$ & 0.115 & 0.32 & 0.543 & 0.02 \\
\hline $1.25-1.33 \mathrm{mi}$ & 0.138 & 0.313 & 0.53 & 0.02 \\
\hline $1.33-1.4 \mathrm{mi}$ & 0.142 & 0.312 & 0.527 & 0.02 \\
\hline $1.4-1.5 \mathrm{mi}$ & 0.145 & 0.311 & 0.525 & 0.02 \\
\hline $1.5-2 \mathrm{mi}$ & 0.158 & 0.304 & 0.517 & 0.019 \\
\hline \multicolumn{5}{|c|}{ Lower LI, MPWR1 } \\
\hline $0-0.25 \mathrm{mj}$ & 0.74 & 0.113 & 0.142 & 0.0051 \\
\hline $0.25-0.33 \mathrm{mi}$ & 0.807 & 0.084 & 0.107 & 0.0038 \\
\hline $0.33-0.4 \mathrm{mi}$ & 0.818 & 0.078 & 0.098 & 0.0035 \\
\hline $0.4-0.5 \mathrm{mi}$ & 0.835 & 0.071 & 0.091 & 0.0032 \\
\hline $0.5-1.25 \mathrm{mi}$ & 0.877 & 0.053 & 0.068 & 0.0024 \\
\hline $1.25-1.33 \mathrm{mi}$ & 0.898 & 0.044 & 0.056 & 0.002 \\
\hline $1.33-1.4 \mathrm{mi}$ & 0.9 & 0.043 & 0.055 & 0.0019 \\
\hline $1.4-1.5 \mathrm{mi}$ & 0.902 & 0.041 & 0.054 & 0.0019 \\
\hline $1.5-2 \mathrm{mi}$ & 0.911 & 0.038 & 0.049 & 0.0017 \\
\hline \multicolumn{5}{|c|}{ Lower LI, MPWR2 } \\
\hline $0-0.25 \mathrm{mi}$ & 0.197 & 0.333 & 0.456 & 0.016 \\
\hline $0.25-0.33 \mathrm{mi}$ & 0.263 & 0.305 & 0.42 & 0.014 \\
\hline $0.33-0.4 \mathrm{mi}$ & 0.283 & 0.296 & 0.407 & 0.014 \\
\hline $0.4-0.5 \mathrm{mi}$ & 0.302 & 0.287 & 0.395 & 0.014 \\
\hline $0.5-1.25 \mathrm{mi}$ & 0.378 & 0.258 & 0.358 & 0.012 \\
\hline $1.25-1.33 \mathrm{mi}$ & 0.424 & 0.236 & 0.33 & 0.011 \\
\hline $1.33-1.4 \mathrm{mi}$ & 0.431 & 0.233 & 0.325 & 0.011 \\
\hline $1.4-1.5 \mathrm{mi}$ & 0.438 & 0.23 & 0.321 & 0.011 \\
\hline $1.5-2 \mathrm{mi}$ & 0.464 & 0.218 & 0.308 & 0.01 \\
\hline \multicolumn{5}{|c|}{ Lower LI, MPWR3 } \\
\hline $0-0.25 \mathrm{mi}$ & 0.051 & 0.355 & 0.573 & 0.022 \\
\hline $0.25-0.33 \mathrm{mi}$ & 0.072 & 0.346 & 0.562 & 0.021 \\
\hline $0.33-0.4 \mathrm{mi}$ & 0.079 & 0.342 & 0.556 & 0.021 \\
\hline
\end{tabular}


Table B.5.4 (continued)

Appendix B Auxiliary Dose Information

\begin{tabular}{|l|c|c|c|c|}
\hline & Cloudshine & Groundshine & $\begin{array}{c}\text { Inhalation } \\
\text { Acute }\end{array}$ & $\begin{array}{c}\text { Resuspension } \\
\text { Acute }\end{array}$ \\
\hline $0.4-0.5 \mathrm{mi}$ & 0.086 & 0.34 & 0.554 & 0.021 \\
\hline $0.5-1.25 \mathrm{mi}$ & 0.117 & 0.329 & 0.537 & 0.02 \\
\hline $1.25-1.33 \mathrm{mi}$ & 0.139 & 0.32 & 0.523 & 0.019 \\
\hline $1.33-1.4 \mathrm{mi}$ & 0.143 & 0.318 & 0.519 & 0.019 \\
\hline $1.4-1.5 \mathrm{mi}$ & 0.146 & 0.316 & 0.517 & 0.019 \\
\hline $1.5-2 \mathrm{mi}$ & 0.159 & 0.312 & 0.51 & 0.019 \\
\hline
\end{tabular}




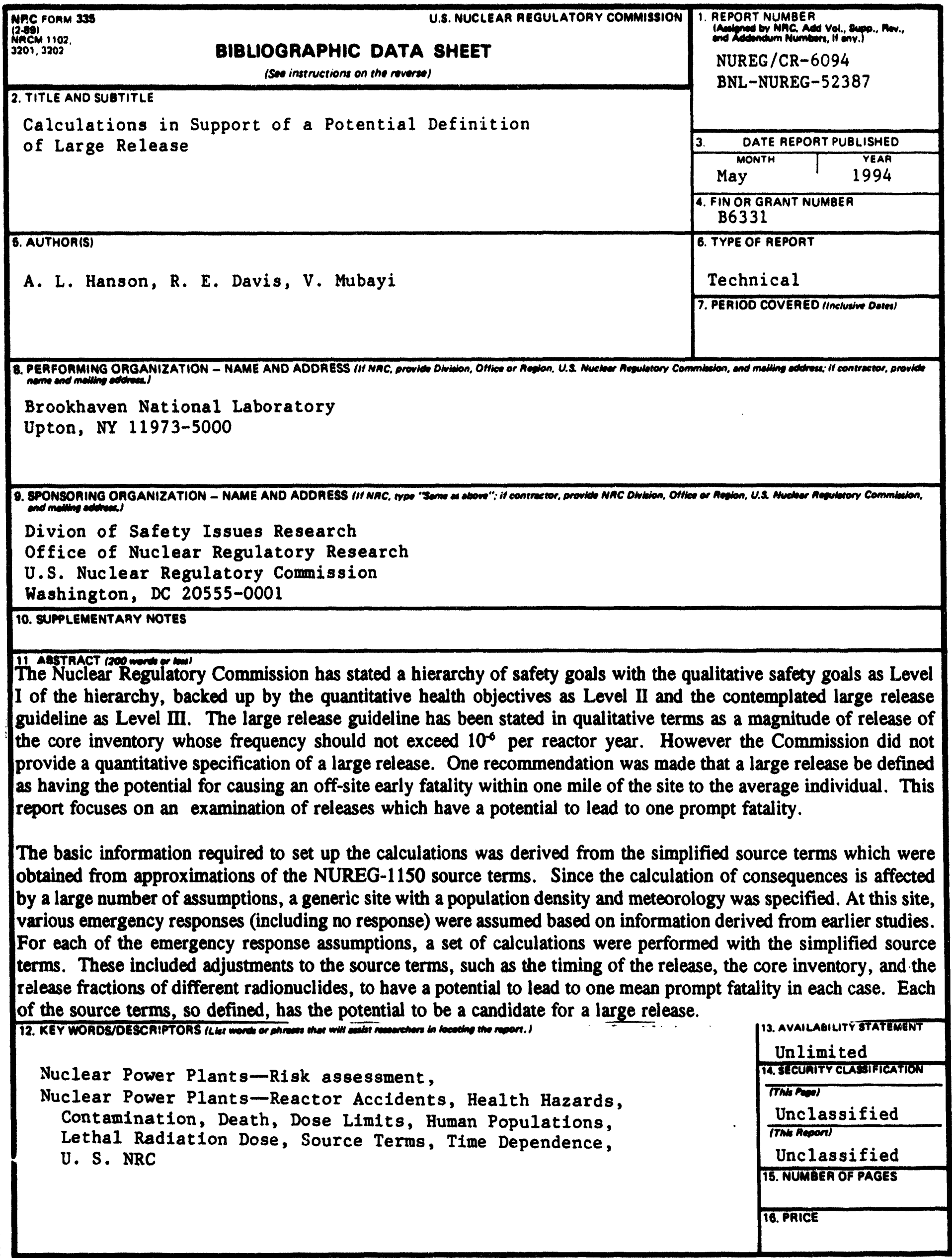



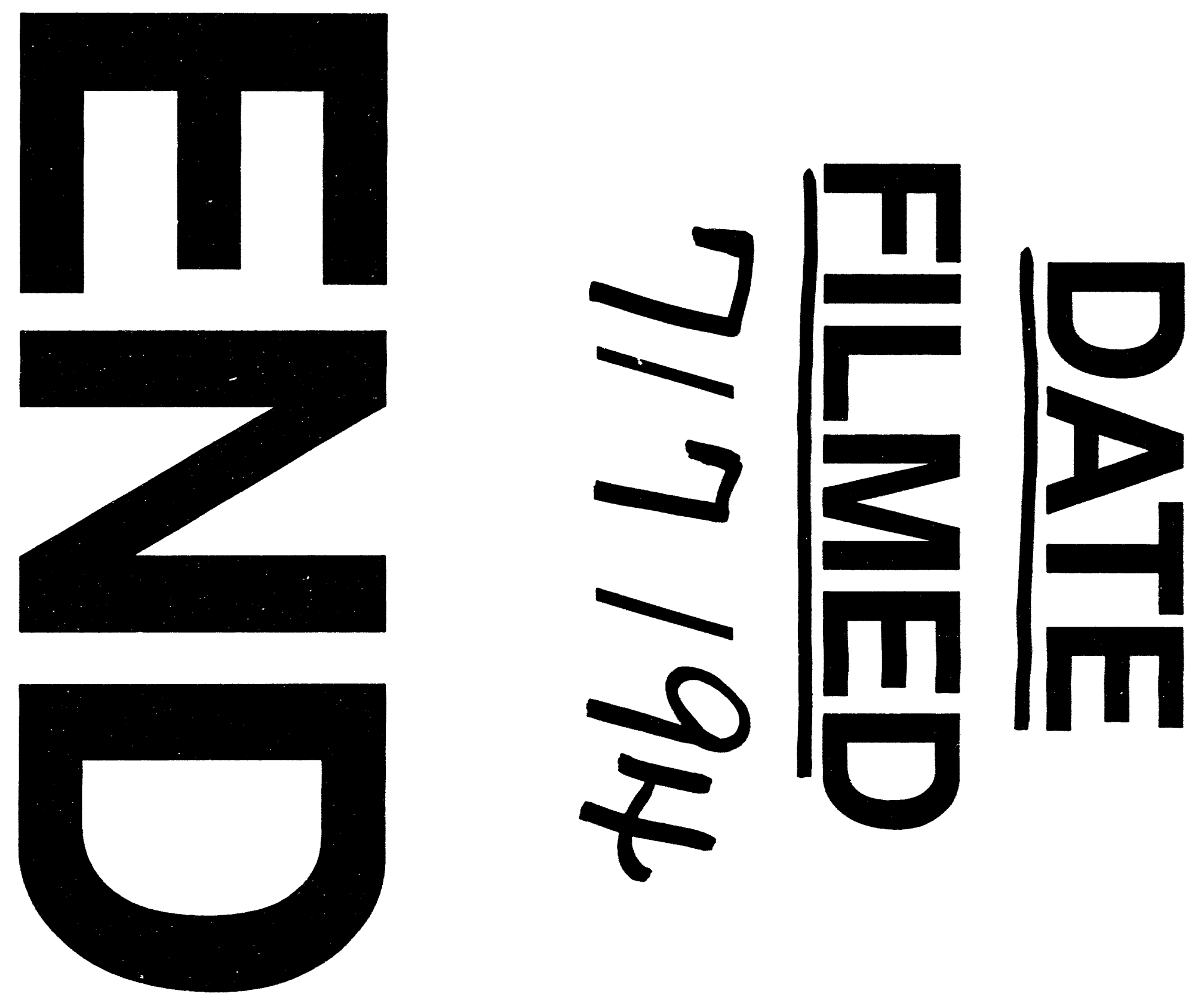